\title{
Towards clinical translation of nanobody-targeted photodynamic therapy
}

Irati Beltrán Hernández 
Towards clinical translation of nanobody-targeted photodynamic therapy $\mathrm{PhD}$ thesis, Utrecht University, the Netherlands

ISBN: 978-94-6423-116-8

Cover design: Irati Beltrán Hernández

Printed by: ProefschriftMaken

The printing of this thesis was (partly) supported by Utrecht Institute of Pharmaceutical Sciences (UIPS), Utrecht, the Netherlands 


\title{
Towards clinical translation of nanobody-targeted photodynamic therapy
}

\author{
Richting klinische vertaling van \\ nanobody-gebaseerde photodynamische therapie \\ (met een samenvatting in het Nederlands)
}

\section{Proefschrift}

ter verkrijging van de graad van doctor aan de Universiteit Utrecht op gezag van de rector magnificus, prof.dr. H.R.B.M. Kummeling, ingevolge het besluit van het college voor promoties in het openbaar te verdedigen op

dinsdag 16 februari 2021 des middags te 2.30 uur

door

\section{Irati Beltrán Hernández}

geboren op 24 maart 1992

te Ibiza, Spanje 


\section{Promotoren:}

Prof. dr. W.E. Hennink

Prof. dr. A.S. Akhmanova

\section{Copromotoren:}

Dr. S. Santos Oliveira

Dr. P.M.P. van Bergen en Henegouwen

The research presented in this thesis was funded by the European Research Council (ERC) under the European Union's Horizon 2020 research and innovation program (grant 677582). 


\section{Contents}

Chapter 1 General Introduction

Chapter 2 Imaging of tumor spheroids, dual-isotope SPECT, and autoradiographic analysis to assess the tumor uptake and distribution of different nanobodies

Chapter 3 Patient-derived head and neck cancer organoids recapitulate EGFR expression levels of respective tissues and are responsive to EGFRtargeted photodynamic therapy

Chapter 4 Molecular targets for anticancer therapies in companion animals and humans: what can we learn from each other?

Chapter 5 Nanobody-targeted photodynamic therapy for the treatment of feline oral carcinoma: a step towards translation to the veterinary clinic

Chapter 6 Preclinical and clinical evidence of immune responses triggered in oncologic photodynamic therapy: clinical recommendations

Chapter 7 The potential of nanobody-targeted photodynamic therapy to trigger immune responses

Chapter 8 Summary and perspectives 

CHAPTER 1

General introduction 


\section{Cancer therapies}

Abnormal and uncontrolled cell growth can occur in any part of the body, making cancer treatment one of the greatest current challenges due to its variety of etiologies, locations, molecular, and genetic features. Accordingly, therapies against cancer come in all shapes and sizes. The mainstream therapeutic approaches are surgery, chemotherapy and radiation therapy, alone or in combination. To illustrate this, as many as half of the cancer patients receive radiotherapy at least once along the course of the disease [1]. These three approaches have led to excellent clinical results for some early stage cancers, such as breast, thyroid, cervical and testicular cancers, all of which present a 5-year relative survival rate above $90 \%$ [2], highlighting the importance of early detection and diagnosis. Despite being clinically successful in some cases, surgery is not always feasible and radiation treatments and chemotherapy are accompanied by side effects that negatively affect treatment tolerability and the patient's quality of life [1].

The oncology landscape is continuously changing, and the list of main anticancer approaches keeps expanding, now including two promising and effective newer modalities known as immunotherapy and targeted therapy. The inclusion in routine practice over the last decade of immune checkpoint inhibitors, a type of immunotherapy, is accountable for the extension of countless lives in patients with a broad range of malignancies, including melanoma, non-small cell lung cancer and renal cell carcinoma $[3,4]$. These inhibitors are monoclonal antibodies (mAbs) that block negative immune regulatory checkpoints on T cells, mainly the CTLA-4 and the PD-1 pathway, thus unleashing antitumor immune responses. This type of immunotherapy has a better toxicity profile than conventional treatments, with only immune-related mild adverse effects. Unfortunately, the reality is that, for most approved indications, only a minority of patients (10-25\%) benefit from a durable response, while primary or acquired resistance due to tumor heterogeneity is the norm [4]. The absence of a baseline immune response in the so-called "cold tumors", mainly the scarcity of tumor-infiltrating lymphocytes, also poses a major hurdle for immunotherapies [5].

One desirable feature of anticancer therapies is selectivity towards tumor cells over normal cells, hence largely sparing healthy tissue. Over the past two decades, the discovery of oncogenes has prompted the development of a great number of targeted cancer therapies [6]. This group of therapies exploits the genetic alterations in cancer cells which lead to changes promoting growth and proliferation. An important pillar of these targeted therapies are mAbs binding a surface antigen, thereby blocking signal transduction and/or recruiting immune effectors [6]. Examples are the mAbs cetuximab and trastuzumab, binding to the epidermal growth factor receptor (EGFR) and the human epidermal growth factor receptor 2 (HER2), respectively. Furthermore, mAbs can provide specific delivery of a cytotoxic payload (e.g. chemotherapeutic drug) in the form of antibody-drug conjugates [7]. Other agents commonly used in targeted therapy are small molecules inhibiting growth signals or angiogenesis, such as the EGFR inhibitor gefitinib. Despite the wide range of targeting molecules, targetable alterations in cancer cells are very diverse, only present in a small subset of patients, and not always tumor-specific [5]. Furthermore, intratumor heterogeneity accounts for the development of resistance not only in the case of immune checkpoint inhibitors, but also in the context of molecular targeted therapies, posing thus the main limitation to the long-term success of these therapies [8].

Each approach has its limitations and it has become evident that combination therapies against multiple cancer hallmarks are preferable to achieve significant longterm benefits beyond good clinical responses [5]. In this regard, combination strategies 
are being employed, such as chemotherapy or kinase inhibitors combined with immune checkpoint blockade for the treatment of lung, breast, gastric and bladder cancer [4]. Combination of immune checkpoint inhibition and radiation therapy is also under investigation in multiple clinical trials, in which radiotherapy may act as an immune adjuvant and effects in untreated tumors have been observed [9]. Interestingly, this adjuvant capacity has been described for chemotherapy as well [10]. Although combination approaches are substantially improving the oncologic scenario, there exists huge variation in survival rates depending on the cancer type (1-98\%) [2]. Therefore, numerous efforts are being put into new and distinct approaches, with great focus on minimizing adverse effects and improving quality of life.

\section{Photodynamic therapy}

Bearing in mind the importance of sparing healthy tissues to reduce adverse effects and improve the quality of life of cancer patients, photodynamic therapy (PDT) has emerged as a minimally invasive and relatively more tumor-selective strategy. Already in 1978, Thomas Dougherty, one of the pioneers of PDT, reported the effective treatment with PDT of 111 carcinoma lesions in 25 patients who had not responded to conventional therapy, including carcinomas of the breast, prostate and squamous cell, among others [11]. These findings were instrumental for the clinical approval of PDT in 1993 for the treatment of bladder cancer in Canada. Since then, its use has expanded to many other oncological indications, including head and neck squamous cell carcinoma (HNSCC), basal cell carcinoma, cutaneous $\mathrm{T}$ cell lymphoma and cancers of the esophagus, biliary tract and lung [12], while numerous clinical trials are ongoing [13].

PDT achieves selectivity by making use of three elements that are harmless on their own but become cytotoxic when combined: a light-activatable compound, so-called photosensitizer (PS), light of the appropriate wavelength and molecular oxygen. A PS is a molecule, commonly with porphyrin-based structure, which can absorb light of a particular wavelength, usually in the visible and near-infrared spectrum $(630-750 \mathrm{~nm})$ [14]. Absorption of light brings the PS to an excited state, which can transfer energy to surrounding molecules. The main photochemical reaction thought to cause major cellular damage is the direct reaction of the excited PS with ground state oxygen $\left({ }^{3} \mathrm{O}_{2}\right)$, resulting in the formation of singlet oxygen $\left({ }^{1} \mathrm{O}_{2}\right)$ [15]. These highly reactive oxygen species (ROS) and other ROS formed in the process (e.g. hydrogen peroxide and superoxide anion) can subsequently damage cellular organelles by reacting with proteins, nucleic acids and lipids. The fact that singlet oxygen is a short-lived species with an intracellular lifetime of $\sim 3 \mu$ s and a radius of action $\sim 150 \mathrm{~nm}$ from its point of production [16], renders PDT controllable as the extent of the damage is limited to where the PS is localized in the illuminated area.

PDT is performed as a two-step procedure that ensures the selectivity of the approach $[12,14]$. First, the PS is administered (typically topical or intravenous) and associates to all cells that encounters due to its relative hydrophobicity. Intravenously administered PS also associates to serum proteins and with time ( $1-4$ days) the PS is mainly retained at the tumor tissue driven by the enhanced permeability and retention effect. This effect is due to the increased extravasation of circulating molecules to the tumor site through the leaky vasculature, and their impaired clearance due to the lack of lymphatics [17]. After tumor disposition of the PS, the second step of the procedure takes place, which involves the local illumination of the tumor to activate the PS uniquely in the area of interest, causing local tissue damage. As such, the main antitumor mechanism of PDT is the direct cytotoxicity of ROS on the malignant tumor cells, but also other PDT effects are known to contribute to the antitumor effects. 
The therapeutic effect of PDT is also dependent on the inflicted damage to the tumor-associated vasculature that occurs when circulating or bound PS is activated within the tumor capillaries. This can lead to disrupted blood flow and/or blood coagulation, resulting in tumor starvation and destruction [18]. In addition, an intriguing third antitumor mechanism can be triggered by PDT, consisting of the development of host antitumor immunity $[19,20]$. The PDT-induced oxidative stress leads to the upregulation of pro-inflammatory molecules in tumor and stroma cells. An example of these are damage-associated molecular patterns (DAMPs), intracellular molecules that when exposed/released by dying cells can be recognized and lead to the activation and maturation of innate immune cells, such as neutrophils, macrophages and dendritic cells. These innate immune cells, recruited during the initial acute inflammation, can phagocyte remaining tumor cells and, particularly dendritic cells, are specialized in tumor antigen presentation to naïve $\mathrm{T}$ cells, thereby triggering the adaptive arm of the immune response. This aspect of PDT is not essential for the initial tumor destruction. However, under the right circumstances, it can trigger systemic antitumor immunity to control metastasis, as well as trigger immune memory to prevent recurrences, as evidenced in the clinic by regression of tumor lesions outside the treated area [21,22]. The three antitumor mechanisms triggered by PDT, i.e. direct tumor cytotoxicity, tumor-associated vasculature damage and antitumor immune responses, are illustrated in Figure 1.

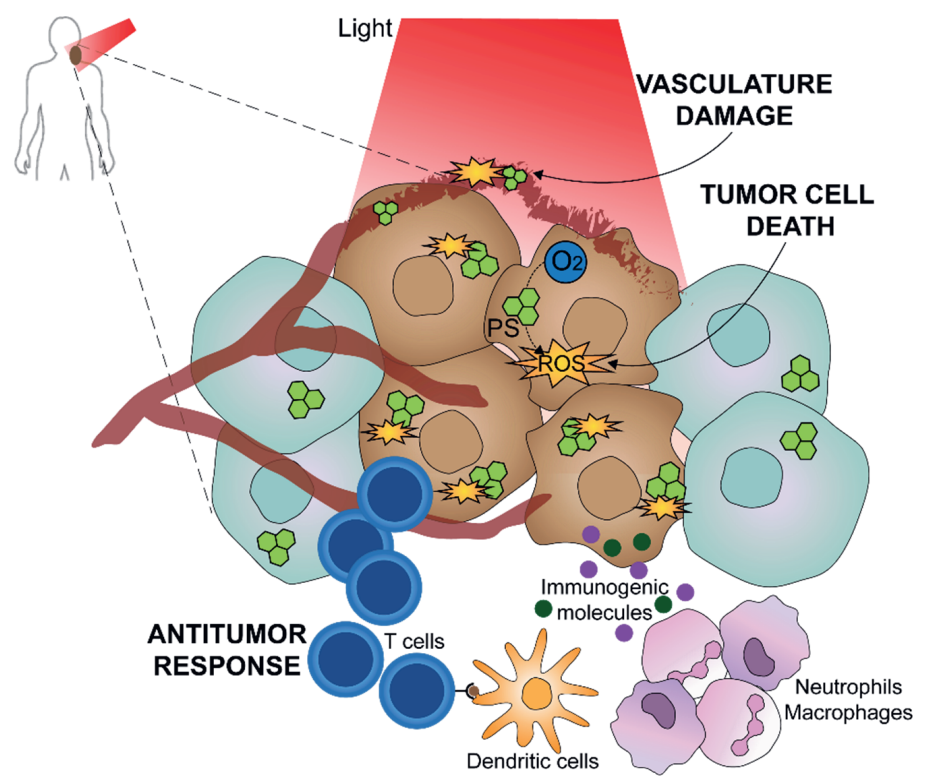

Figure 1 Antitumor mechanisms of PDT.

PDT has yielded good clinical responses together with good cosmetic outcome and improved quality of life. For instance, early complete response rates can be about $90 \%$ in nodular and superficial basal cell carcinoma and nearly $100 \%$ in early stage HNSCC $[12,23]$. Nonetheless, PDT is still underutilized in the clinic, and several limiting factors prevent its position beside other standard care treatments [12]. A significant problem of most approved PSs is the prolonged skin photosensitivity caused by the slow clearance of the PS deposited in the skin, forcing patients to avoid exposure to strong light for days/weeks after treatment. Other drawbacks are the limited tissue penetration of light 
and the heterogeneous PS distribution through tumors [24], compromising the treatment of large and deep tumors. In this regard, light in the near-infrared range presents the best tissue penetrating capacity and novel photosensitizers tend to exploit this. Damage of peripheral normal tissue is also a concern when utilizing PDT against large tumors. Besides, hypoxic tumor areas also limit the therapeutic efficacy. The complex light dosimetry and unintended usage of suboptimal PDT protocols are also a point of concern [25].

\section{Targeted photodynamic therapy}

Attempts to improve the tumor-selectivity of PDT are ongoing, with the idea of improving therapeutic efficacy as well as reducing side effects, including both off-target effects and those related to high PS dosage. One interesting strategy is the use of targeting molecules to deliver the PS to the cells of interest. In particular in the form of antibodyphotosensitizer conjugates, an approach referred to as photoimmunotherapy (PIT) and first described in 1983 [26]. In this way, the selectivity achieved in a passive manner with the conventional PDT approach, becomes now molecularly targeted. More recently, PIT has evolved towards the use of water-soluble PSs, facilitating direct conjugation to the $\mathrm{mAb}$ without the need of linkers and/or intermediary reactions [27]. Moreover, by using water-soluble PSs, the chances of non-specific association are reduced because the PS now fully depends on the mAb to associate to cells. The potency of PIT to induce direct tumor cytotoxicity and even immune protection has been well-described in vitro [28,29] and in mouse models [30-32], in which EGFR proves to be a promising target. In fact, a conjugate consisting of the commercial anti-EGFR mAb cetuximab and the water-soluble PS IRDye700DX is now under evaluation in a phase III clinical trial for the treatment of HNSCC (NCT03769506). Of note, this PS has an absorption maximum in the nearinfrared spectrum $(689 \mathrm{~nm})$, thus increasing the depth of light penetration, and can be used in parallel as a fluorescent tool for molecular cancer imaging. In PIT, the pharmacokinetics of the PS are now guided by a relatively large mAb $(\sim 150 \mathrm{kDa})$. Such a molecular size is accompanied by heterogeneous tumor distribution and long circulation times (half-life of IgG1 30 days [33]), thus not improving the PS distribution throughout the tumor, nor the prolonged photosensitivity post-treatment observed with conventional PDT.

One step further in the journey to improve the conventional PDT approach is the conjugation of the PS IRDye700DX to nanobodies (NBs), giving rise to nanobodytargeted PDT (NB-PDT). NBs are the smallest naturally occurring antigen binding domains which derive from the variable domain of heavy chain Abs, first discovered in Camelids in 1993 [34]. The small size of nanobodies $(\sim 15 \mathrm{kDa})$ and high binding affinities bring about advantages over their larger antibody counterparts, especially in the context of PDT. For instance, a more rapid and homogeneous distribution throughout tumors is reported for NBs in comparison to mAbs [35,36]. Over the last 7 years, the potency and specificity of NB-PDT has been proven in vitro for a wide range of membrane receptors, including EGFR [37], HER2 [38], hepatocyte growth factor receptor (c-Met) [39], and the G protein-coupled receptor (GPCR) US28 [40]. In addition, in mouse models, the induction of extensive tumor necrosis [41], significant tumor regression [38], and damage to tumor-associated vasculature [42] have been described. Contrary to PIT, nanobody-photosensitizer (NB-PS) conjugates present rapid renal clearance and short serum half-life ( 90 minutes), due to their small size $[41,43]$. Conjugates are thereby rapidly cleared from circulation when unbound, enabling faster illumination after PS administration ( $1-2$ hours) and potentially reducing the chances of 
photosensitivity. NB-PDT is so far a promising alternative which distinguishes itself from current PDT approaches by a more favorable pharmacokinetic profile.

\section{HNSCC and EGFR}

HNSCC accounts for $90 \%$ of the cancers in the head and neck area and has one of the highest incidences worldwide, becoming the sixth most common cancer [2]. HNSCC is a remarkably heterogeneous disease arising from the squamous cells in the outer mucosa layer, lining the throat, nasal and oral cavity. Next to heavy alcohol consumption and tobacco use as risk factors, the human papillomavirus (HPV) and Epstein Barr Virus (EBV) are linked with the development of this cancer as well [44]. Treatment of early stage disease is relatively successful; however, at the moment of diagnosis, about half of the patients already present locally advanced HNSCC, while around $10 \%$ of these present metastasizing tumors [45]. Treatment consists mainly of chemoradiotherapy, preceded by surgical resection when feasible [44]. Of note, this heavy use of multimodal approaches profoundly impacts the quality of life of the patients. Overall, the five-year survival rate for these patients is $40-50 \%$, and for tumors of all stages [46]. Unfortunately, recurrence occurs in around half of the patients and the prognosis is poor for those patients with recurrent or metastatic HNSCC, with an overall survival of 10-13 months [45].

Broadening the therapeutic scenario, the anti-EGFR mAb cetuximab was approved in combination with radiotherapy for the treatment of locally or regionally advanced HNSCC in 2006. Currently, it is also being used as a single agent as well as in combination with platinum-based chemotherapy for patients with recurrent or metastatic HNSCC [47]. Immune checkpoint inhibitors have also proven valuable in the fight against HNSCC. Their use was approved in 2016 for treatment of recurrent/metastatic disease, especially in PD-L1 positive tumors [45,47]. Interestingly, research combining both EGFR-targeting and immunotherapy is already underway [46]. Despite advances, the $40-50 \%$ mortality rate for HNSCC has remained unchanged over the past decades, illustrating the need for alternative and improved treatments and/or combinations. Following the same trend as with mainstream chemoradiotherapy and surgery, PDT yields excellent clinical outcome in early stage HNSCC, but treatment of advanced tumors remains a challenge [12].

One well-characterized driver of molecular pathogenesis of HNSCC are alterations in EGFR, also known as ErbB1 or HER1. This transmembrane tyrosine kinase receptor is one of four receptors constituting the ErbB family and is the most frequently overexpressed and/or activated in HNSCC. EGFR is highly expressed in epithelial cells and, as such, overexpressed in up to $90 \%$ of HNSCC malignancies [48]. Besides EGF, other autocrine ligands can also activate EGFR by binding to the extracellular region, such as transforming growth factor alpha, amphiregulin and epiregulin. Upon ligand binding, homodimerization of EGFR or heterodimerization with other ErbB family members is induced, followed by cross-phosphorylation of the intracellular tyrosine kinase domains and subsequent downstream signaling. Here, EGFR functions as an upstream point activating signaling pathways which promote tumor progression, via the control of cell proliferation, migration, survival and angiogenesis [49]. Therefore, it is evident that abnormal activation of this receptor would lead to a malignant phenotype and, indeed, its overexpression correlates with more aggressive disease and poor prognosis [47]. EGFR is the more mature target to date in HNSCC, and cetuximab is so far the only targeted therapy approved for its treatment. Nonetheless, intrinsic and acquired resistance poses a major obstacle for long term responses. Other anti-EGFR $\mathrm{mAbs}$ and kinase inhibitors are being investigated in clinical trials to treat HNSCC, but clinical success is modest so far $[44,49]$. In the context of PDT, EGFR also seems to be a 
valuable target. PIT using cetuximab-IRDye700DX is currently in phase III for the treatment of locoregional recurrent HNSCC in patients who have failed at least two lines of therapy (NCT03769506). To date, PIT has yielded improved response rates over standard of care therapies, in such a heavily pretreated population with recalcitrant disease [50].

\section{Translational approaches}

The need for novel therapeutic options against cancer comes hand in hand with the testing and development of more accurate and clinically relevant models. Preliminary toxicity, efficacy and pharmacokinetics of any anticancer compound are always first examined in vitro and in animal experiments. The broad use of immortalized tumor-derived cell lines in the first steps of preclinical development is justified by the low-cost and simple handling, but it is obvious that these genetically homogeneous cell lines fall behind regarding physiological relevance [51]. The growth of cell lines in a 3D structure, either in suspension or embedded in a matrix, adds one level of complexity which can more precisely reflect a solid tumor mass, including nutrient and oxygen gradients and cell to cell/matrix interactions [52]. However, tumor phenotype and heterogeneity, capillary extravasation and systemic clearance are still not represented in these models. To better represent tumor structure and phenotype, tumor organoids have emerged as $3 \mathrm{D}$ selforganizing structures grown from patient-derived tumor tissue obtained by surgical resections or biopsies [53]. These organoids grow in extracellular matrix gels, recapitulate the morphologic, genetic and phenotypic features of the tumor tissue of origin and have been successfully employed for drug screening and personalized medicine approaches [53]. Additionally, organoids can also be grown from adjacent healthy tissue to enable assessment of tumor-specific responses. Even though organoids are a phenocopy of the original tumor, the interface with the surrounding vasculature and stroma, as well as normal mechanical cues are still lacking. These features are reproduced, to some extent, in the more recent technology known as microfluidic organs-on-chips [54]. Using a multichannel microfluidic cell culture chip, patient-derived tumor cells can be grown lined by normal cells from the same primary organ, while sustaining vascular perfusion. As with organoids, this technology can be utilized to advance personalized medicine.

It is clear that in vitro tumor models have gained in complexity, but animal experimentation is always a step in the further development of promising therapies. A major experimental tool is the mouse xenograft model, consisting of injection of human tumor cell lines or primary patient-derived tumor tissue into immunocompromised mice, predominantly subcutaneously, thus in an ectopic manner that does not reproduce the tissue-specific microenvironment [55,56]. This aspect is improved in orthotopic models in which the tumor is xenografted at the same organ from which the original tumor derives, better mimicking tumor growth and metastasis. Alternatively, tumors can be induced by a carcinogen, genetic engineering or injection of murine tumor cells in immunocompetent mice [56], offering a more suitable platform for investigating immunomodulatory therapies, as PDT has proven to be. Still, all these animal models are limited in their mirroring of the intricate human carcinogenesis and physiology, thus often failing to reproduce metastasis, host immunity, or development of treatment resistance. In addition, their utility is hindered by the critical genetic, molecular, cellular and immunologic differences between species [57]. Although these models are still useful to assess the extent of antitumor effect of the approach, promising preclinical approaches generally fail when translated to human trials. Particularly for cancer research, the success rate of translation from animal studies to clinical use is as little as $3.4 \%$, the lowest among therapeutic disciplines [57,58]. 
Concerning tumor microenvironment, from the simplistic 2D cell culture to complex mouse models, all approaches have their limitations. Moreover, with the recent advent of immunomodulatory therapies, models which more faithfully reproduce the human immunological features are of paramount importance. The discipline of comparative oncology includes the evaluation of promising anticancer strategies in companion animals with naturally occurring tumors, with the idea of translating to human cancer patients [59]. By nature, this is a win-win scenario, in which better therapeutic options can arise for both veterinary and human patients. The study of these spontaneous cancers in animals with intact immunity exposed to the same environmental factors as humans is of remarkable translational significance. One benefit of this approach is that the shorter lifespan of companion animals allows rapid data collection. Moreover, the lack of standard of care options for these animals presents the opportunity of, within reason, initiating therapeutic trials at any point in non-pretreated populations, which is usually not the case for human patients [60]. Several cancer types in companion animals are considered as relevant models of the multifaceted human malignancies. These exhibit great clinical and molecular similarities to the human counterparts, including the heterogeneity of the disease, interactions between tumor and immune cells, development of treatment resistance, metastasis and clinical outcome. Examples of these natural occurring models are canine and feline mammary carcinoma [60,61], canine melanoma [62], canine brain tumors [63], canine osteosarcoma [64], canine bladder cancer [65] and feline oral squamous cell carcinoma [66]. The possibility of helping these animals with generally otherwise poor outcomes and the knowledge gained to ensure human patients are given treatments likely to succeed, highlight the value of assessing new strategies in the veterinary clinic.

\section{Scope of the thesis}

This thesis focuses on positioning NB-PDT one step closer to the clinic, particularly towards the use of EGFR-targeted NB-PDT to treat HNSCC. To achieve this, three aspects were mainly investigated: the NB format that yields rapid and homogeneous tumor accumulation, the use of clinically relevant in vitro HNSCC models of the human and feline malignancy to facilitate transition, and the immune responses as part of the antitumor effects of NB-PDT which can be determinant for clinical success of the treatment.

In Chapter 2, the effect that specificity and molecular size have on the tumor uptake and distribution of NBs, compared to mAbs, was assessed in 3D spheroids, and further studied in mice with different NB formats. Moving towards the field of targeted PDT and the use of clinically relevant 3D models, Chapter 3 explores the use EGFRtargeting NB-PS and $\mathrm{mAb}$-PS conjugates for PDT in organoids grown from both HNSCC patient material and correspondent adjacent normal tissue. NB-PDT has already proven selective and potent in HNSCC cell lines as well as in an orthotopic mouse model of oral carcinoma, but the use of patient-derived organoids gives a better idea of the likelihood of NB-PDT to be successful in the clinical setting, especially regarding clinically relevant target expression levels. With this goal of clinical translation of NB-PDT in mind, Chapter 4 presents an overview of the most well-characterized canine and feline spontaneous solid cancers and main targetable molecules, to aid the development of targeted therapies with reasonable translation potential for the human counterpart malignancy. In this context, efforts were put in the development of a NB-PS conjugate for the treatment of cats with oral squamous cell carcinoma (OSCC), a naturally occurring model of HNSCC. This is presented in Chapter 5, where an EGFR-targeting, species cross-reactive NB-PS conjugate was characterized and its potency and selectivity for NB- 
PDT evaluated on feline OSCC cell lines. Also relevant for clinical translation is the understanding of the antitumor immune responses triggered by PDT, which can be determinant for the long-term success of the treatment. As such, Chapter $\mathbf{6}$ reviews the available preclinical and clinical evidence of antitumor immunity post-PDT. Insights are given on the possible combination therapies to exploit this aspect of PDT and recommendations are given to aid further understanding of these events in the clinical setting. Chapter 7 follows on this in the context of NB-PDT, where the first signs of immune stimulation after NB-PDT were investigated in vitro, including the release of DAMPs and cytokines, followed by dendritic cell maturation and $\mathrm{T}$ cell activation. Lastly, in Chapter 8, the findings of this thesis are summarized and future perspectives regarding NB-PDT are discussed.

\section{References}

1. Citrin, D.E. Recent developments in radiotherapy. N. Engl. J. Med. 2017, 377, 1065-1075.

2. $\quad$ Miller, K.D.; Nogueira, L.; Mariotto, A.B.; Rowland, J.H.; Yabroff, K.R.; Alfano, C.M.; Jemal, A.; Kramer, J.L.; Siegel, R.L. Cancer treatment and survivorship statistics, 2019. CA. Cancer J. Clin. 2019, 69, 363-385.

3. Wolchok, J. Putting the Immunologic Brakes on Cancer. Cell 2018, 175, 1452-1454.

4. Schoenfeld, A.J.; Hellmann, M.D. Acquired Resistance to Immune Checkpoint Inhibitors. Cancer Cell 2020, 37, 443-455.

5. Zugazagoitia, J.; Guedes, C.; Ponce, S.; Ferrer, I.; Molina-Pinelo, S.; Paz-Ares, L. Current Challenges in Cancer Treatment. Clin. Ther. 2016, 38, 1551-1566.

6. Lee, Y.T.; Tan, Y.J.; Oon, C.E. Molecular targeted therapy: Treating cancer with specificity. Eur. J. Pharmacol. 2018, 834, 188-196.

7. Zuo, P. Capturing the Magic Bullet: Pharmacokinetic Principles and Modeling of Antibody-Drug Conjugates. AAPS J. 2020, 22, 105 .

8. Marusyk, A.; Janiszewska, M.; Polyak, K. Intratumor Heterogeneity: The Rosetta Stone of Therapy Resistance. Cancer Cell 2020, 37, 471-484.

9. Schaue, D.; Mcbride, W.H. Opportunities and challenges of radiotherapy for treating cancer. Nat. Rev. Clin. Oncol. 2015, 12, 527-540.

10. Rivera Vargas, T.; Apetoh, L. Danger signals : Chemotherapy enhancers? Immunol. Rev. 2017, 280, 175-193.

11. Dougherty, T.J.; Kaufman, J.E.; Goldfarb, A.; Weishaupt, K.R.; Boyle, D.; Mittleman, A. Photoradiation Therapy for the Treatment of Malignant Tumors. Cancer Res. 1978, 38, 2628-2635.

12. van Straten, D.; Mashayekhi, V.; de Bruijn, H.S.; Oliveira, S.; Robinson, D.J. Oncologic photodynamic therapy: Basic principles, current clinical status and future directions. Cancers (Basel). 2017, 9, 1-54.

13. U.S. National Institutes of Health ClinicalTrials.gov Available online: https://clinicaltrials.gov/ (accessed on May 1, 2020).

14. Dolmans, D.E.J.G.J.; Fukumura, D.; Jain, R.K. Photodynamic therapy for cancer. Nat Rev Cancer 2003, 3, 380-387.

15. Foote, C. Definition of type I and type II photosensitized oxidation. Photochem. Photobiol. 1991, 54,659 .

16. Sinksb, B.W.P.L.E.; Breitenbacha, T.; Schacka, N.B.; Vinogradovb, S.A.; Ogilby, P.R. Single Cell Responses to Spatially-Controlled Photosensitized Production of Extracellular Singlet Oxygen. Photochem. Photobiol. 2011, 87, 1077-1091.

17. Maeda, H. Toward a full understanding of the EPR effect in primary and metastatic tumors as well as issues related to its heterogeneity. Adv. Drug Deliv. Rev. 2015, 91, 3-6.

18. Abels, C. Targeting of the vascular system of solid tumours by photodynamic therapy (PDT). 
Photochem. Photobiol. Sci. 2004, 3, 765-771.

19. Garg, A.D.; Nowis, D.; Golab, J.; Agostinis, P. Photodynamic therapy: Illuminating the road from cell death towards anti-tumour immunity. Apoptosis 2010, 15, 1050-1071.

20. Beltrán Hernández, I.; Yu, Y.; Ossendorp, F.; Korbelik, M.; Oliveira, S. Preclinical and Clinical Evidence of Immune Responses Triggered in Oncologic Photodynamic Therapy: Clinical Recommendations. J. Clin. Med. 2020, 9, 333.

21. Kabingu, E.; Oseroff, A.; Wilding, G.; Gollnick, S. Enhanced systemic immune reactivity to a Basal cell carcinoma associated antigen following photodynamic therapy. Clin. cancer Res. 2009, 15, 4460-4466.

22. Thong, P.S.P.; Ong, K.W.; Goh, N.S.G.; Kho, K.W.; Manivasager, V.; Bhuvaneswari, R.; Olivo, M.; Soo, K.C. Photodynamic-therapy-activated immune response against distant untreated tumours in recurrent angiosarcoma. Lancet Oncol. 2007.

23. Agostinis, P.; Berg, K.; Cengel, K..; Foster, T..; Girotti, A..; Gollnick, S..; Hahn, S..; Hamblin, M..; Juzeniene, A.; Kessel, D.; et al. Photodynamic Therapy of cancer: an update. CA Cancer J Clin. 2011, 61, 250-281.

24. Mallidi, S.; Anbil, S.; Bulin, A.; Obaid, G.; Ichikawa, M.; Hasan, T. Beyond the Barriers of Light Penetration: Strategies, Perspectives and Possibilities for Photodynamic Therapy. Theranostics 2016, 6, 2458-2487.

25. Pogue, B.W.; Elliott, J.T.; Kanick, S.C.; Davis, S.C.; Samkoe, K.S.; Maytin, E. V; Pereira, S.P.; Hasan, T.; 1 Revisiting photodynamic therapy dosimetry : reductionist \& surrogate approaches to facilitate clinical success. Phys. Med. Biol. 2016, 61, R57-R89.

26. Mew, D.; Wat, C.; Towers, G.; Levy, J. Photoimmunotherapy: treatment of animal tumors with tumor-specific monoclonal antibody-hematoporphyrin conjugates. J. Immunol. 1983, 130, $1473-$ 1477.

27. Kobayashi, H.; Choyke, P.L. Near-Infrared Photoimmunotherapy of Cancer. Acc. Chem. Res. 2019, 52, 2332-2339.

28. Ogawa, M.; Tomita, Y.; Nakamura, Y.; Lee, M.; Lee, S.; Tomita, S.; Nagaya, T.; Sato, K.; Yamauchi, T.; Trepel, B.; et al. Immunogenic cancer cell death selectively induced by near infrared photoimmunotherapy initiates host tumor immunity. Oncotarget 2017, 8, 10425-10436.

29. Sato, K.; Watanabe, R.; Hanaoka, H.; Harada, T.; Nakajima, T.; Kim, I.; Paik, C.H.; Choyke, P.L.; Kobayashi, H. Photoimmunotherapy: Comparative effectiveness of two monoclonal antibodies targeting the epidermal growth factor receptor. Mol. Oncol. 2014, 8, 620-632.

30. Nagaya, T.; Friedman, J.; Maruoka, Y.; Ogata, F.; Okuyama, S.; Clavijo, P.E.; Choyke, P.L.; Allen, C.; Kobayashi, H. Host immunity following near-infrared photoimmunotherapy is enhanced with PD-1 checkpoint blockade to eradicate established antigenic tumors. Cancer Immunol. Res. 2019, 7, 401-413.

31. Mitsunaga, M.; Nakajima, T.; Sano, K.; Kramer-Marek, G.; Choyke, P.L.; Kobayashi, H. Immediate in vivo target-specific cancer cell death after near infrared photoimmunotherapy. $B M C$ Cancer 2012, 12, 1 .

32. Mitsunaga, M.; Ogawa, M.; Kosaka, N.; Rosenblum, L.T.; Choyke, P.L.; Kobayashi, H. Cancer Cell-Selective In Vivo Near Infrared Photoimmunotherapy Targeting Specific Membrane Molecules. Nat. Med. 2011, 17, 1685-1691.

33. Mankarious, S.; Lee, M.; Fischer, S.; Pyun, K.; Ochs, H.; Oxelius, V.; Wedgwood, R. The halflives of $\mathrm{IgG}$ subclasses and specific antibodies in patients with primary immunodeficiency who are receiving intravenously administered immunoglobulin. J. Lab. Clin. Med. 1988, 112, 634-640.

34. Hamers-Casterman C Atarhouch, T.; Muyldermans, S.; Robinson, G.; Hamers, C.; Songa, E.; Bendahman, N.; Hamers, R. Naturally occurring antibodies devoid of light chains. Nature 1993, 363, 446-448.

35. Oliveira, S.; Van Dongen, G. a M.S.; Stigter-Van Walsum, M.; Roovers, R.C.; Stam, J.C.; Mali, W.; Van Diest, P.J.; Van Bergen En Henegouwen, P.M.P. Rapid visualization of human tumor xenografts through optical imaging with a near-infrared fluorescent anti-epidermal growth factor receptor nanobody. Mol. Imaging 2012, 11,33-46.

36. Kijanka, M.; Warnders, F.J.; El Khattabi, M.; Lub-De Hooge, M.; Van Dam, G.M.; Ntziachristos, V.; De Vries, L.; Oliveira, S.; Van Bergen En Henegouwen, P.M.P. Rapid optical imaging of human 
breast tumour xenografts using anti-HER2 VHHs site-directly conjugated to IRDye $800 \mathrm{CW}$ for image-guided surgery. Eur. J. Nucl. Med. Mol. Imaging 2013, 40, 1718-1729.

37. Heukers, R.; van Bergen en Henegouwen, P.M.P.; Oliveira, S. Nanobody-photosensitizer conjugates for targeted photodynamic therapy. Nanomedicine Nanotechnology, Biol. Med. 2014, 10, 1441-1451.

38. Deken, M.M.; Kijanka, M.M.; Beltrán Hernández, I.; Slooter, M.D.; de Bruijn, H.S.; van Diest, P.J.; van Bergen en Henegouwen, P.M.; Lowik, C.W.; Robinson, D.J.; Vahrmeijer, A.L.; et al. Nanobody-targeted photodynamic therapy induces significant tumor regression of trastuzumabresistant HER2-positive breast cancer, after a single treatment session. J. Control. Release 2020, $323,269-281$.

39. Heukers, R.; Mashayekhi, V.; Ramirez-Escudero, M.; de Haard, H.; Verrips, T.C.; van Bergen en Henegouwen, P.M.P.; Oliveira, S. VHH-Photosensitizer Conjugates for Targeted Photodynamic Therapy of Met-Overexpressing Tumor Cells. Antibodies (Basel) 2019, 8, 26.

40. De Groof, T.W.M.; Mashayekhi, V.; Shu Fan, T.; Bergkamp, N.D.; Sastre Torano, J.; Van Senten, J.R.; Heukers, R.; Smit, M.J.; Oliveira, S. Nanobody-Targeted Photodynamic Therapy Selectively Kills Viral GPCR-Expressing Glioblastoma Cells. Mol. Pharm. 2019, 16, 3145-3156.

41. Van Driel, P.B.A.A.; Boonstra, M.C.; Slooter, M.D.; Heukers, R.; Stammes, M.A.; Snoeks, T.J.A.; De Bruijn, H.S.; Van Diest, P.J.; Vahrmeijer, A.L.; Van Bergen En Henegouwen, P.M.P.; et al. EGFR targeted nanobody-photosensitizer conjugates for photodynamic therapy in a pre-clinical model of head and neck cancer. J. Control. Release 2016, 229, 93-105.

42. de Bruijn, H.S.; Mashayekhi, V.; Schreurs, T.J.L.; van Driel, P.B.A.A.; Strijkers, G.J.; van Diest, P.J.; Lowik, C.W.G.M.; Seynhaeve, A.L.B.; Hagen, T.L.M. ten; Prompers, J.J.; et al. Acute cellular and vascular responses to photodynamic therapy using EGFR-targeted nanobody-photosensitizer conjugates studied with intravital optical imaging and magnetic resonance imaging. Theranostics 2020, 10, 2436-2452.

43. Roovers, R.C.; Laeremans, T.; Huang, L.; De Taeye, S.; Verkleij, A.J.; Revets, H.; De Haard, H.J.; van Bergen En Henegouwen, P.M.P. Efficient inhibition of EGFR signalling and of tumour growth by antagonistic anti-EGFR Nanobodies. Cancer Immunol. Immunother. 2007, 56, 303-317.

44. Klein, J.D.; Grandis, J.R. The molecular pathogenesis of head and neck cancer. Cancer Biol. Ther. 2010, 9, 1-7.

45. Cohen, E.E.W.; Bell, R.B.; Bifulco, C.B.; Burtness, B.; Gillison, M.L.; Harrington, K.J.; Le, Q.; Lee, N.Y.; Leidner, R.; Lewis, R.L.; et al. The Society for Immunotherapy of Cancer consensus statement on immunotherapy for the treatment of squamous cell carcinoma of the head and neck (HNSCC). J. Immunother. Cancer 2019, 7.

46. Saba, N.F.; Chen, Z.G.; Haigentz, M.; Bossi, P.; Rinaldo, A.; Rodrigo, J.P.; Antti, A.M.; Takes, R.P.; Strojan, P.; Vermorken, J.B.; et al. Targeting the EGFR and Immune Pathways in Squamous Cell Carcinoma of the Head and Neck (SCCHN): Forging a New Alliance. Mol. Cancer Ther. 2019, $18,1909-1916$

47. Santuray, R.T.; Johnson, D.E.; Grandis, J.R. New Therapies in Head and Neck Cancer. Trends in Cancer 2018, 4, 385-396.

48. Bossi, P.; Resteghini, C.; Paielli, N.; Licitra, L.; Pilotti, S.; Perrone, F. Prognostic and predictive value of EGFR in head and neck squamous cell carcinoma. Oncotarget 2016, 7, 74362-74379.

49. Jue Xu, M.; Johnson, D.E.; Grandis, J.R. EGFR-Targeted Therapies in the Post-Genomic Era. Cancer Metastasis Rev. 2017, 36, 463-473.

50. David M. Cognetti; Johnson, J.M.; Curry, J.M.; Mott, F.; Kochuparambil, S.T.; McDonald, D.; Fidler, M.J.; Stenson, K.; Vasan, N.R.; Razaq, M.; et al. Results of a phase 2a, multicenter, openlabel, study of RM-1929 photoimmunotherapy (PIT) in patients with locoregional, recurrent head and neck squamous cell carcinoma (rHNSCC). J. Clin. Oncol. 2019, 37, 6014.

51. Sharma, S. V.; Haber, D.A.; Settleman, J. Cell line-based platforms to evaluate the therapeutic efficacy of candidate anticancer agents. Nat. Rev. Cancer 2010, 10, 241-53.

52. Katt, M.E.; Placone, A.L.; Wong, A.D.; Xu, Z.S.; Searson, P.C. In Vitro Tumor Models : Advantages, Disadvantages, Variables, and Selecting the Right Platform. Front. Bioeng. Biotechnol. 2016, 4 .

53. Clevers, H. Modeling Development and Disease with Organoids. Cell 2016, 165, 1586-1597. 
54. Sontheimer-Phelps, A.; Hassell, B.A.; Ingber, D.E. Modelling cancer in microfluidic human organs-on-chip. Nat. Rev. Cancer 2019, 19, 65-81.

55. Jung, J.; Sook Seol, H.; Chang, S. The Generation and Application of Patient-Derived Xenograft Model for Cancer Research. Cancer Res. Treat. 2018, 50, 1-10.

56. Galuschka, C.; Proynova, R.; Roth, B.; Augustin, H.G.; Muller-Decker, K. Models in Translational Oncology: A Public Resource Database for Preclinical Cancer Research. Cancer Res. 2017, 77, 2557-2564.

57. Mak, I.W.Y.; Evaniew, N.; Ghert, M. Lost in translation: animal models and clinical trials in cancer treatment. Am. J. Transl. Res. 2014, 6, 114-118.

58. Heem Wong, C.; Wei Siah, K.; Lo, A.W. Estimation of clinical trial success rates and related parameters. Biostatistics 2019, 20, 273-286.

59. Riccardo, F.; Aurisicchio, L.; Impellizeri, J.A.; Cavallo, F. The importance of comparative oncology in translational medicine. Cancer Immunol. Immunother. 2015, 64, 137-148.

60. Cannon, C.M. Cats, Cancer and Comparative Oncology. Vet. Sci. 2015, 2, 111-126.

61. Abdelmegeed, S.M.; Mohammed, S. Canine mammary tumors as a model for human disease (Review). Oncol. Lett. 2018, 15, 8195-8205.

62. Prouteau, A.; André, C. Canine Melanomas as Models for Human Melanomas: Clinical, Histological, and Genetic Comparison. Genes (Basel). 2019, 10.

63. Hicks, J.; Platt, S.; Kent, M.; Haley, A. Canine brain tumours: a model for the human disease? Vet. Comp. Oncol. 2015, 15, 252-272.

64. Simpson, S.; Dunning, M.D.; de Brot, S.; Grau-Roma, L.; Mongan, N.P.; Rutland, C.S. Comparative review of human and canine osteosarcoma: morphology, epidemiology, prognosis, treatment and genetics. Acta Vet. Scand. 2017, 59.

65. Knapp, D.W.; Dhawan, D.; Ramos-Vara, J.A.; Ratliff, T.L.; Cresswell, G.M.; Utturkar, S.; Sommer, B.C.; Fulkerson, C.M.; Hahn, N.M. Naturally-Occurring Invasive Urothelial Carcinoma in Dogs, a Unique Model to Drive Advances in Managing Muscle Invasive Bladder Cancer in Humans. Front. Oncol. 2020, 9, 1493.

66. Wypij, J.M. A naturally occurring feline model of head and neck squamous cell carcinoma. Patholog. Res. Int. 2013, 2013. 
General introduction 



\section{CHAPTER 2}

\section{Imaging of tumor spheroids, dual-isotope SPECT, and autoradiographic analysis to assess the tumor uptake and distribution of different nanobodies}

Irati Beltrán Hernández ${ }^{1,2, *}$, Rene Rompen ${ }^{3, *}$, Raffaella Rossin ${ }^{4}$, Katerina T. Xenaki $^{2}$, Eugene A. Katrukha ${ }^{2}$, Klaas Nicolay ${ }^{3}$, Paul van Bergen en Henegouwen ${ }^{2}$, Holger Grüll ${ }^{3,4,5}$ and Sabrina Oliveira ${ }^{1,2}$

\footnotetext{
${ }^{1}$ Pharmaceutics, Department of Pharmaceutical Sciences, Faculty of Science, Utrecht University, Utrecht, the Netherlands

${ }^{2}$ Cell Biology, Neurobiology and Biophysics, Department of Biology, Faculty of Science, Utrecht University, Utrecht, the Netherlands

${ }^{3}$ Department of Biomedical Engineering, Eindhoven University of Technology, Eindhoven, the Netherlands

${ }^{4}$ Department of Minimally Invasive Healthcare, Philips Research Laboratories, Eindhoven, the Netherlands

${ }^{5}$ Department of Diagnostic and Interventional Radiology, University of Cologne, Faculty of Medicine and University Hospital of Cologne, Cologne, Germany

* These authors contributed equally to this work
} 


\begin{abstract}
Recent studies have shown rapid accumulation of nanobodies (NBs) in tumors and fast clearance of the unbound fraction, making NBs exceptional tracers for cancer imaging. In this study, we investigate the combination of in vitro imaging of tumor spheroids, in vivo dual-isotope single-photon emission computed tomography (SPECT), and ex vivo autoradiographic analysis of tumors to efficiently, and with few mice, assess the tumor uptake and distribution of different NBs. The irrelevant NB R2 $(16 \mathrm{kDa})$ and the EGFRtargeted NBs 7D12 (16 kDa) and 7D12-R2 (32 kDa) were investigated. Confocal microscopy was used to study the penetration of the NBs into A431 tumor spheroids over time, using the anti-EGFR monoclonal antibody $(\mathrm{mAb})$ cetuximab $(150 \mathrm{kDa})$ as a reference. Dual-isotope $\left[{ }^{111} \mathrm{In}\right] \mathrm{DOTA}-\mathrm{NB} /\left[{ }^{177} \mathrm{Lu}\right] \mathrm{DOTA}-\mathrm{NB}$ SPECT was used for longitudinal imaging of multiple tracers in the same animal bearing A431 tumor xenografts. Tumor sections were analyzed using autoradiography. No binding of the irrelevant NB was observed in spheroids, whereas for the specific tracers an increase in the spheroid's covered area was observed over time. The NB 7D12 saturated the spheroid earlier than the larger, 7D12-R2. Even slower penetration was observed for the large $\mathrm{mAb}$. In vivo, the tumor uptake of 7D12 was 19-fold higher than R2 after co-injection in the same animal, and 2.5-fold higher than 7D12-R2 when co-injected. 7D12-R2 was mainly localized at the rim of tumors, while 7D12 was found to be more evenly distributed. This study demonstrates that the combination of imaging of tumor spheroids, dual-isotope SPECT, and autoradiography of tumors is effective in comparing tumor uptake and distribution of different NBs. Results were in agreement with published data, highlighting the value of monomeric NBs for tumor imaging, and re-enforcing the value of these techniques to accurately assess the most optimal format for tumor imaging. This combination of techniques requires a lower number of animals to obtain significant data and can accelerate the design of novel tracers.
\end{abstract}




\section{Introduction}

The discovery and design of new molecular imaging tracers are of great importance for early detection and diagnosis of cancer. Therefore, a wide range of tumor specific tracers have been developed for the imaging of solid tumors. Besides the metabolic positron emission tomography (PET) tracer 2-deoxy-2-[18F]fluoro-D-glucose, proteins that bind to different tumor antigens are commonly used in the clinic for cancer imaging, and can vary in size by at least two orders of magnitude (100-102 nm) [1,2]. In particular, monoclonal antibodies (mAbs, $150 \mathrm{kDa}$ ) are the most frequently used in the clinic [3]. However, $\mathrm{mAbs}$ are not ideal for tumor imaging due to long time intervals between tracer administration and imaging, and limited and heterogeneous distribution of the $\mathrm{mAb}$ inside tumors [4]. Molecular size is one of the well-recognized factors affecting tumor uptake and distribution [5]. Thus, many efforts have been made to develop smaller tracers to allow more rapid imaging and to reduce the radioactive burden for patients in case of nuclear imaging. Among antibody fragments, nanobodies (NBs, $16 \mathrm{kDa}$ ) have emerged as an attractive alternative format for molecular imaging. NBs consist of the variable region of the heavy-chain antibodies found in camelids and cartilaginous fish [6]. Fast penetration and homogeneous distribution into tumors, as well as high-contrast imaging rapidly after NB injection, are some of the advantages of using NBs for imaging purposes $[7,8]$. In fact, clinical evaluation of NBs for PET imaging has thus far been encouraging [9].

Developing a nuclear tracer for the clinic requires in vitro characterization of the tracer, followed by in vivo assessment of its biodistribution and tumor targeting properties. To aid and accelerate this process, efforts are being made in order to develop in vitro and in vivo models, as well as imaging techniques for the preclinical setting [10]. One of such in vitro models is spheroids three-dimensional (3D) cell cultures. In this model, cell to cell contact and interactions are maintained in all three dimensions in order to grow and resemble an in vivo tumor. In contrast to the commonly used 2D cultures, these spheroids simulate the in vivo tumor environment and maintain most of the characteristics present in tumors [11]. Advances in imaging techniques also aid the development of imaging tracers. Dual-isotope single-photon emission computed tomography (SPECT) is a non-invasive imaging strategy used in several medical fields, such as cardiology [12] and neurology [13]. This imaging technique allows distinction of radiolabeled compounds both in space and over time, making possible the simultaneous detection of multiple tracers in a single subject. Importantly, dual-isotope indium111/lutetium-177 SPECT has been already used as a tool to evaluate small peptide tracers in the preclinical setting [14], and its use is extended to the NB field in the present study.

In this study, we investigate the use of in vitro 3D tumor spheroid models in combination with in vivo dual-isotope SPECT imaging, and ex vivo autoradiographic analysis of tumors, to efficiently assess the tumor uptake and distribution of different NB formats, while using a reduced number of animals. First, we have compared the irrelevant NB R2 (16 kDa), raised against the azo-dye Reactive Red, with the EGFR-targeted monomeric NB 7D12 (16 kDa), to assess the effect of binding specificity on tumor uptake. Thereafter, we have compared the tumor uptake and distribution of 7D12 with the dimeric NB 7D12-R2 (32 kDa), which has similar binding affinity to EGFR but with twice the molecular weight. This study demonstrates that this combination of in vitro, in vivo, and ex vivo imaging techniques is effective in evaluating the tumor uptake and distribution of the different NB formats, further encouraging the use of the monomeric format as a tracer for imaging of solid tumors. 


\section{Materials and Methods}

\section{Cell Culture and Spheroid Formation}

The A431 cell line (epidermoid carcinoma) was purchased from the American Type Culture Collection (ATCC) and cultured in high-glucose Dulbecco's Modified Eagle's Medium (Lonza) supplemented with $10 \%$ fetal calf serum (GE Healthcare), $100 \mu \mathrm{g} / \mathrm{ml}$ streptomycin, and $100 \mathrm{U} / \mathrm{ml}$ penicillin (Invitrogen). To grow spheroids, the wells of an 8well plate (Nunc Lab-Tek II Chambered Slide 8-wells plates; Thermo Scientific) were coated with a thin layer of Matrigel (Corning) and incubated at $37^{\circ} \mathrm{C}$ for $20 \mathrm{~min}$ to allow polymerization. A suspension of 20,000 cells was added per well and incubated at $37^{\circ} \mathrm{C}$ for 1 week. The medium was refreshed every other day.

\section{In Vivo Studies}

Six-to-nine weeks old Balb/C nude female mice were purchased from Charles Rivers. A431 cells $(2 \times 106$ cell $/ 100 \mu 1$ sterile PBS $)$ were subcutaneously injected in the left shoulder of the mice. When tumors reached a size of 100-300 mm3, mice were used for imaging or biodistribution studies. The animal welfare committee of the Maastricht University (The Netherlands) approved all animal studies. Experiments were performed according to the U.S. National Institutes of Health principles of laboratory animal care and the Dutch national law "Wet op de Dierproeven" (Std 1985, 336).

\section{Proteins and Labeling}

NBs R2 and 7D12 have been previously described [15,16]. The dimeric NB 7D12-R2 consists of these two NBs connected by a linker of 10 amino acids, being 7D12 the only EGFR binding unit. All NBs were produced and purified as previously described [17]. Cetuximab was purchased from Merck.

For in vitro studies, proteins were conjugated to the fluorophore Alexa Fluor 647 NHS ester (Life Technologies), purified, and analyzed by SDS-PAGE as described in [16] for the photosensitizer IRDye700DX, except that the labeling reaction took place for $1.5 \mathrm{~h}$. The concentration of the final conjugate and degree of labeling were calculated by measuring absorbance at 280 and $689 \mathrm{~nm}$, as described in the manufacturer's manual.

For in vivo studies, NBs were conjugated to $5 \mathrm{eq} \mathrm{p}-\mathrm{SCN}-\mathrm{Bn}$-DOTA (Macrocyclics) in DMSO. The reaction was carried out for $30 \mathrm{~min}$ at $37^{\circ} \mathrm{C}$ at $\mathrm{pH}$ 9. PD-10 columns $(5$ $\mathrm{kDa}$ MWCO, GE Healthcare) were used to purify the DOTA-NB conjugates. The concentration of each conjugate in PBS was measured by UV at $280 \mathrm{~nm}$ (NanoDrop, Thermo Fisher) or with a BCA assay (Thermo Fisher). The DOTA-NB conjugates were analyzed by SDS-PAGE on $12.5 \%$ homogeneous gels under non-reducing conditions. The conjugates were radiolabeled with $\left[{ }^{111} \mathrm{In}\right] \mathrm{Cl} 3$ or $\left[{ }^{177} \mathrm{Lu}\right] \mathrm{Cl} 3$ (Mallinckrodt and Perkin Elmer, respectively), in $0.2 \mathrm{M}$ HEPES $\mathrm{pH}$ or $0.2 \mathrm{M}$ ammonium acetate $\mathrm{pH} 5.5$, respectively. The mixtures were incubated for $1 \mathrm{~h}$ at $37^{\circ} \mathrm{C}$ followed by a DTPA challenge, to remove loosely bound radiometal, and size exclusion purification (Zeba desalting cartridges, $7 \mathrm{kDa}$ MWCO, Thermo Fisher). Labeling yields were determined by radioTLC using a phosphor imager (FLA-7000, Fujifilm). Aliquots of the labeling mixtures were applied on TLC-SG strips (Varian Inc.) which were eluted with $200 \mathrm{mM}$ EDTA in saline. In these conditions, the radiolabeled NB remains at the origin while free In-111 or Lu-177 migrates with a $0.9 \mathrm{Rf}$. In order to adjust the specific activity, the radiolabeled NBs were mixed with the respective unlabeled NB. For in vivo studies, radiolabeled NBs were added with gentisic acid to a $5 \mathrm{mg} / \mathrm{ml}$ final concentration to protect the conjugates from radiolysis. 


\section{Binding Affinity of Labeled Proteins}

The apparent binding affinity of the labeled tracers was assessed with A431 cells as previously described [16]. Briefly, cells in 96-well plates were incubated for $2 \mathrm{~h}$ at $4{ }^{\circ} \mathrm{C}$ with a concentration range of NBs in DMEM (without phenol red, and with $25 \mathrm{mM}$ Hepes and $1 \% \mathrm{BSA}, \mathrm{pH} 7.2$ ). After washing off unbound NBs, plates were scanned with the Odyssey scanner at $700 \mathrm{~nm}$ in the case of the Alexa 647-labeled proteins. For the radiolabeled tracers, the content of the wells was finally dissolved in $0.5 \% \mathrm{SDS}$, buffered and radioactivity measured in a $\gamma$-counter (Wizard 1480, Perkin Elmer) using a dualisotope protocol with cross-contamination correction. Apparent affinity (KD) was calculated with GraphPad Prism 7.

\section{Fluorescence Confocal Microscopy}

Spheroids were incubated with $25 \mathrm{nM}$ of Alexa 647-labeled tracers for different time intervals at $37^{\circ} \mathrm{C}$. After each incubation time, unbound conjugate was washed away and spheroids were fixed in $4 \%$ paraformaldehyde (Merck). Background fluorescence was quenched with $100 \mathrm{mM}$ glycine (Sigma-Aldrich) and spheroids were permeabilized using $0.5 \%$ Triton X-100 (Sigma-Aldrich). Staining of the nuclei was performed with DAPI (Roche). In some cases, F-actin was stained with phalloidin-488 (Thermo Fisher). Finally, the slides were mounted with SlowFade Gold (Invitrogen). Imaging was performed using a confocal laser scanning microscope LSM700 (Carl Zeiss Microscopy GmbH) with a $\times 40$ oil objective (EC Plan-NeoFluar $\times 40 / 1.3$ Oil DIC). Pictures of spheroids were taken through the middle $\mathrm{z}$-stack section.

Confocal images were analyzed with the Image $\mathrm{J}$ software. The plugin Radial Profile was used to obtain the profile of fluorescence intensity of Alexa 647 along the radius of the spheroid. This profile was adjusted to the radius of the spheroid since overall the radii varied only $\pm 12.5 \mu \mathrm{m}$. Data were normalized and depicted as the fluorescence intensity from the rim to the core of the spheroid using GraphPad Prism 7. Profile plots display the fluorescence intensity profile of one representative spheroid per time point. The area under the curve (AUC) was calculated from the fluorescence profiles and plotted against time. A minimum of eight spheroids from at least two independent experiments were analyzed per time point.

\section{In Vivo SPECT/CT Imaging and Ex Vivo Biodistribution Studies}

Imaging studies were performed with groups of four A431 tumor-bearing mice injected with [ $\left.{ }^{111} \mathrm{In}\right]$ DOTA-7D12 (ca. $10 \mathrm{MBq} / 100 \mu \mathrm{l}$ ), $\left[{ }^{111} \mathrm{In}\right] \mathrm{DOTA}-7 \mathrm{D} 12 /\left[{ }^{177} \mathrm{Lu}\right] \mathrm{DOTA}-7 \mathrm{D} 12$, $\left[{ }^{111} \mathrm{In}\right]$ DOTA-7D12/[ $\left.{ }^{177} \mathrm{Lu}\right] \mathrm{DOTA}-\mathrm{R} 2$, or $\left[{ }^{111} \mathrm{In}\right] \mathrm{DOTA}-7 \mathrm{D} 12 /\left[{ }^{177} \mathrm{Lu}\right] \mathrm{DOTA}-7 \mathrm{D} 12-\mathrm{R} 2$ (ca. $10 \mathrm{MBq}$ In-111 and $40 \mathrm{MBq}$ Lu-177 combined in $100 \mu \mathrm{l}$ ). All mice received a similar dose of NB (ca. $0.16 \mu \mathrm{mol} / \mathrm{kg}$ total NB dose). For imaging studies, the animals were anesthetized with isoflurane and imaging started right after injection of labeled NBs. Imaging was performed with a small-animal SPECT/CT scanner (NanoSPECT, Bioscan) equipped with four detectors and converging nine-pinhole collimators $(1.4 \mathrm{~mm}$ pinhole diameter, $1 \mathrm{~mm}$ resolution). Windows of a relative peak width of $10 \%$ were positioned around 171 and $245 \mathrm{keV}$ for In-111, and $208 \mathrm{keV}$ for Lu-177. In total 180 projections were acquired over three rotations. Immediately after imaging (ca. $3 \mathrm{~h}$ post injection), the animals were euthanized, tissues and organs of interest were collected, and radiation was quantified with a $\gamma$-counter. The collected samples included blood, tumor, heart, lung, liver, spleen, pancreas, kidneys, bladder, intestines, muscle, femur, and brain. Because of the upper detection limit of the $\gamma$-counter, only half of each tumor and one middle slice 
from the kidneys were quantified. The other half of the tumor was snap frozen and used for digital autoradiography.

One group of tumor-bearing mice was injected with a similar dose of $\left[{ }^{111} \mathrm{In}\right] \mathrm{DOTA}-$ 7D12/[ $\left.{ }^{177} \mathrm{Lu}\right]$ DOTA-7D12 (ca. $0.16 \mu \mathrm{mol} / \mathrm{kg}$ total NB) labeled at a lower molar activity (2.5 MBq for In-111 and $10 \mathrm{MBq}$ for Lu-177 combined in $100 \mu \mathrm{l}$, injected in awake mice) and was euthanized $1 \mathrm{~h}$ post-injection. The animals were dissected and organs and tissues of interest were harvested and measured in a $\gamma$-counter.

\section{SPECT/CT Image Reconstitution}

SPECT images were reconstructed using HiSpect software (SciVis) with an ordered subset expectation maximization (OSEM) iterative reconstruction algorithm. CT images were reconstructed using an exact cone beam FPB algorithm with a Shepp Logan filter $(100 \%)$. SPECT and CT images were fused in InVivoScope (BioScan). CT images were used to select regions of interest in muscle, liver, and bone. For tumor and kidney, regions were selected based on the $75 \%$ threshold value of the maximum intensity pixel values per each organ.

\section{Autoradiographic $\beta$-Imaging}

Frozen tumor sections of $10 \mu \mathrm{m}$ were mounted on microscopy slides, thawed, covered with scintillation foil, and imaged with a BetaIMAGER DFine (Biospace Lab). Radioactive signal was separated based on decay-rates and scaling was adjusted to match the difference in $\beta$-particle emission of In-111 to Lu-177. Analysis and quantification of the images were performed with M3Vision (Biospace Lab). At least two images were quantified per each tumor.

\section{Statistical Analysis}

In vitro data were analyzed with GraphPad Prism 7 using one-way ANOVA with Tukey post-hoc test to compare between groups. For the in vivo data, significance between tumor or kidney uptake of different groups was calculated with a 2-tailed t test or one-way ANOVA. A value of $\mathrm{p} \leq 0.05$ was considered significant. Statistical significance was depicted as $* \mathrm{p} \leq 0.05, * * \mathrm{p} \leq 0.01, * * * \mathrm{p} \leq 0.001$, and $* * * * \mathrm{p} \leq 0.0001$.

\section{Results}

\section{Alexa 647-Labeled Proteins}

The degree of labeling (DOL) for the anti-EGFR NBs 7D12 and 7D12-R2 was 0.55. In the case of cetuximab and R2, the DOL was 1.44 and 0.3 respectively. The remaining free fluorophore in the protein conjugate was in all cases less than $10 \%$. All conjugates were first analyzed by SDS-PAGE to verify the purity and molecular size, and their binding affinity was assessed on monolayer cell cultures (Figure S1a,b). The KD of the EGFR-targeted tracers remained in the low nanomolar range and was comparable to the unconjugated counterparts $[17,18]$ (Figure S1e). Importantly, the binding affinity of 7D12 and 7D12-R2 was similar, while R2 did not show a specific binding to A431 cells.

\section{Penetration and Distribution of Proteins into Tumor Spheroids}

A431 spheroids showed progressive growth over time and consisted of a compact mass of cells with cortical actin distribution (Figure 1a). After more than 10 days in culture, the integrity of the spheroids was compromised, yielding empty spaces of different sizes. 
Therefore, during our experiments, we used 7-days-old tumor spheroids with a diameter of $150-200 \mu \mathrm{m}$.

The bound EGFR-targeted NBs were observed deeper into spheroids over time, as indicated by the clear fluorescent signal on the cell membrane (Figure 1b). In contrast, the irrelevant NB R2 could not be detected inside the spheroids at any time point. Fluorescence signal of 7D12 and 7D12-R2 was detected in the core of spheroids as early as $15 \mathrm{~min}$ after tracer addition, and this signal increased over time until reaching the same values as at the rim after 2 and $4 \mathrm{~h}$, respectively (Figure 1b,c). The area under the curve (AUC) was determined from the fluorescence profiles of the tracers along the radius of the spheroids and plotted over time (Figure 1d). Some delay in tumor accumulation was observed for the dimeric 7D12-R2 tracer when compared with the monomeric 7D12 (Figure 1c,d), while a more apparent delay was seen for cetuximab, which started to be observed in the core only after $3 \mathrm{~h}$ (Figure 1c). The delay of the $\mathrm{mAb}$ is more clearly visualized by the small slope of the spheroid coverage over time (Figure 1d).

\section{Radiolabeled Proteins}

The In-111 or Lu-177 labeling yield was comparable for all NBs, reaching more than 80 $\%$ incorporation of In-111 or Lu-177 in all cases. After size exclusion purification, all tracers presented less than $5 \%$ free radiometal. Labeled NBs were analyzed on SDSPAGE gel for their size and their apparent affinities were assessed by binding assays on monolayer cell cultures (Figure S1c,d). The apparent affinity of the NBs remained in the low nanomolar range after radiolabeling (Figure S1e). No specific binding to cells was observed for radiolabeled R2. The fact that molecular size and binding affinity remain comparable for each particular NB, regardless of the fluorophore/isotope, suggests no detrimental effect of the different conjugations on binding properties, though direct comparisons between in vitro and in vivo data should be made carefully. Importantly, the main comparisons to be made are between the pairs of NBs that are subjected to the same labeling.

\section{Testing of Dual-Isotope SPECT and Comparison with Ex Vivo Biodistribution}

Tumor uptake of the NB 7D12 was clearly visible at 1 and $3 \mathrm{~h}$ post-injection in mice injected with the single-isotope [ ${ }^{111}$ In]DOTA-7D12 or with the combination of In-111 or Lu-177 radiolabeled 7D12, showing no differences along time (Figure 2a). Biodistribution of the tracers was determined both by SPECT imaging and by $\gamma$-counting of the respective organs (Figure $2 b$ ). Both techniques revealed a similar tumor uptake of the differently radiolabeled 7D12 over time, thus confirming that the distribution is not influenced by the radiolabel. A strong correlation was found for the uptake values in all examined organs measured by single and dual-isotope SPECT $(\mathrm{R} 2=0.97$ for In-111 and $\mathrm{R} 2=0.97$ for $\mathrm{Lu}-177)$. Apart from the tumors, radioactivity uptake of both tracers was mainly observed in the kidneys (Figure 2b). Only there, differences were observed as the uptake for the dual-isotope group, detected by both SPECT imaging and $\gamma$-counting, was consistently higher than the single-isotope group. These differences may arise from the presence of crosstalk effects, particularly of Lu-177 in the acquisition window of In-111. Furthermore, metal chelates can have different renal clearance or uptake since these are also degraded in the kidney, and therefore different metal chelates can behave significantly different. 

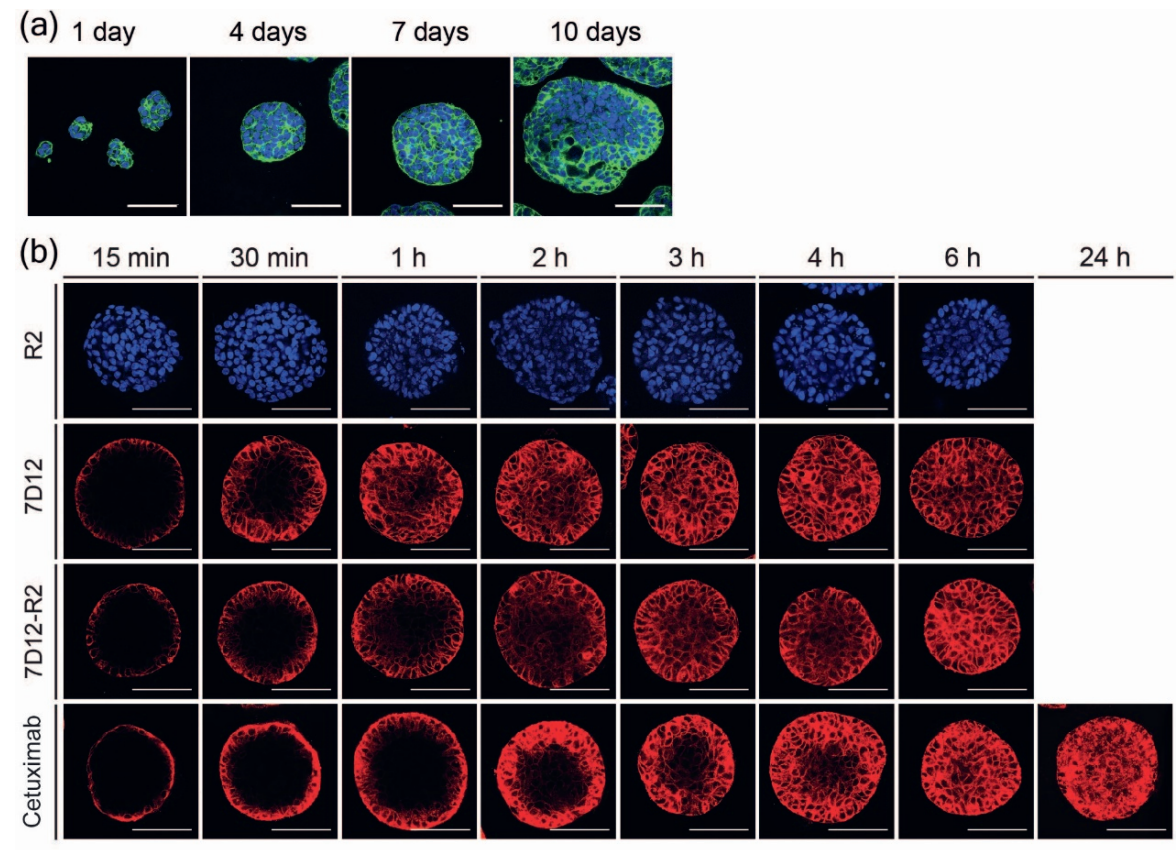

(c)

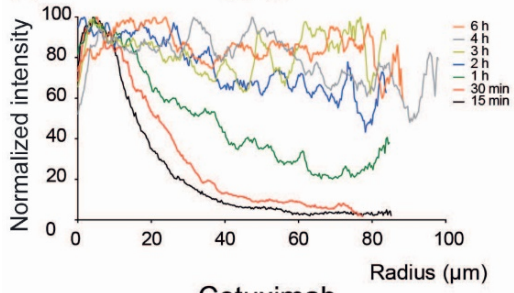

Cetuximab

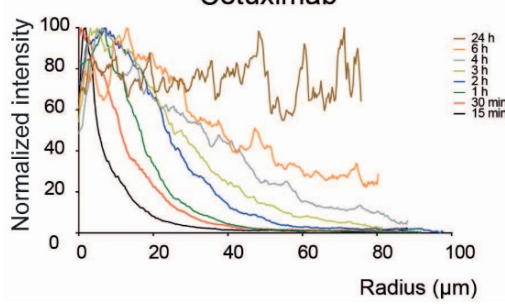

7D12-R2
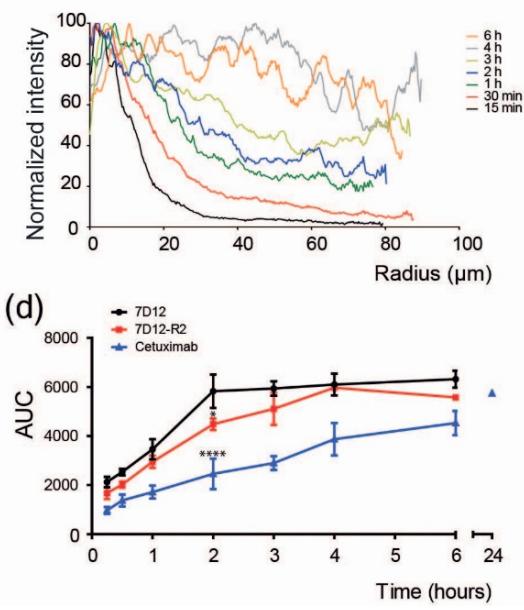

Figure 1 Penetration and distribution of labeled proteins into tumor spheroids over time. (a) The growth of A431 spheroids was monitored over 10 days by taking confocal images at the middle section of spheroids. The spheroids were visualized by staining the nucleus of the cells with DAPI (blue) and the F-actin filaments with phalloidin-488 (green). (b) Confocal images of spheroids that were fixed after incubation with the Alexa 647-labeled proteins R2, 7D12, 7D12-R2, and cetuximab at defined time points (binding shown in red). In the case of R2, no Alexa 647 signal was detected bound to cells and, therefore, DAPI images of the same spheroids are shown instead, to depict the integrity of the spheroids. All images were linearly adjusted to the same level of contrast. Scale bar, $100 \mu \mathrm{m}$. (c) The fluorescence signal along the radius of spheroids was quantified from confocal images and displayed from the rim to the center of the spheroid. Graphs show one representative profile per time point and tracer. (d) The area under the curve of the 
fluorescence profiles was calculated and plotted against time as an estimation of the protein penetration into the spheroids in time. Statistical significance of the observed differences between each group and the 7D12 group was determined for the $2 \mathrm{~h}$ time point (mean $\pm \mathrm{SD}$ ). ${ }^{*} \mathrm{p} \leq 0.05$; $* * * * \mathrm{p} \leq 0.0001$.

(a)

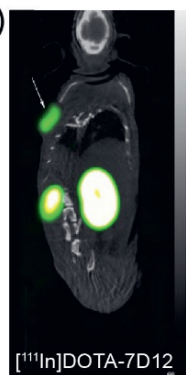

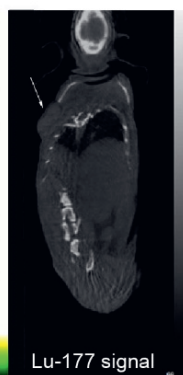
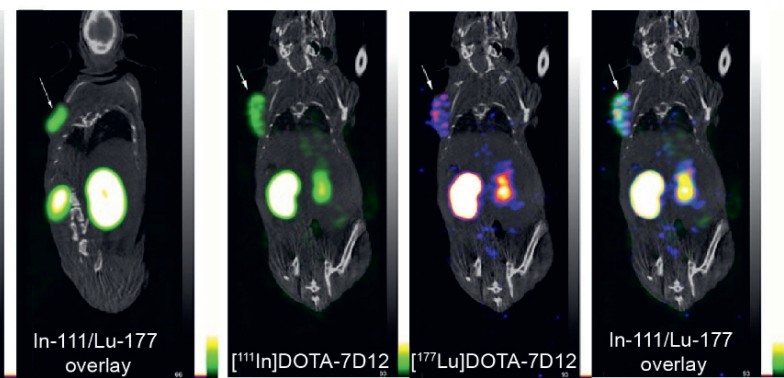

(b)
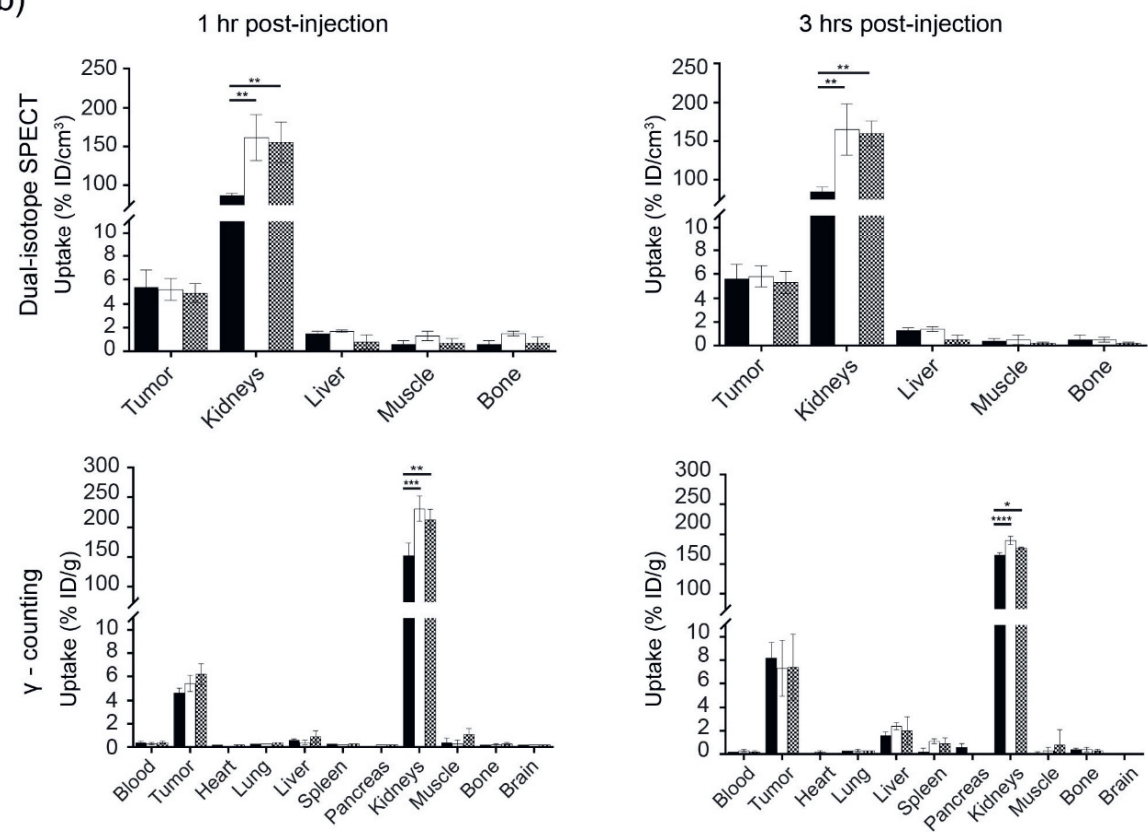

${ }^{11}$ In]DOTA-7D12 (single-isotope)

$\square\left[{ }^{111} \operatorname{In}\right]$ DOTA-7D12 (dual-isotope)

[ [ ${ }^{177}$ Lu]DOTA-7D12 (dual-isotope)

Figure 2 Effect of radionuclide on imaging and biodistribution. (a) SPECT/CT coronal images of A431 tumor bearing mice $1 \mathrm{~h}$ after injection of $\left[{ }^{111} \mathrm{In}\right] \mathrm{DOTA}-7 \mathrm{D} 12$ (left) and $3 \mathrm{~h}$ after injection of [ ${ }^{111}$ In]DOTA-7D12/[ $\left.{ }^{177} \mathrm{Lu}\right]$ DOTA-7D12 (right). In-111 signal and Lu-177 signal were merged with CT images. Tumor location is indicated by arrows. (b) Biodistribution data obtained by single and dual-isotope SPECT imaging and $\gamma$-counting $1 \mathrm{~h}$ and $3 \mathrm{~h}$ after injection of $\left[{ }^{111}\right.$ In]DOTA-7D12 or $\left[{ }^{111} \mathrm{In}\right]$ DOTA-7D12/[ $\left.{ }^{177} \mathrm{Lu}\right]$ DOTA-7D12. Statistical significance of the observed differences between the kidney uptake of each dual-isotope group and the single-isotope group was determined (mean $\pm \mathrm{SD}$ ). ${ }^{*} \mathrm{p} \leq 0.05,{ }^{* *} \mathrm{p} \leq 0.01,{ }^{* * *} \mathrm{p} \leq 0.001$, and ${ }^{* * * * \mathrm{p}} \leq 0.0001$. 


\section{Effect of Binding Specificity and of Tracer Molecular Size on Tumor Uptake and Biodistribution}

Tumor uptake was determined by both SPECT imaging and $\gamma$-counting after 1 and $3 \mathrm{~h}$ after tracer injection (Figure 3a, Figure S2a). The biodistribution of 7D12 was similar in all imaged animals for both time points. As calculated from SPECT imaging after 1 and $3 \mathrm{~h}, 7 \mathrm{D} 12$ presented a 19-fold higher tumor uptake than R2 after co-injection, whereas the examined organs showed a ratio close to 1 (Figure $3 b$, Figure S2b). When $\left[{ }^{111} \mathrm{In}\right] \mathrm{DOTA}-7 \mathrm{D} 12$ and $\left[{ }^{177} \mathrm{Lu}\right] \mathrm{DOTA}-7 \mathrm{D} 12-\mathrm{R} 2$ were co-injected, tumor uptake was 2.5fold higher for the monomeric NB 7D12, both after 1 and $3 \mathrm{~h}$, as measured by SPECT imaging. Importantly, SPECT imaging also showed a 2-3 times higher liver uptake of 7D12-R2 than 7D12. In addition, also the $\gamma$-counting results of blood and other organs (heart, pancreas, muscle, and lung) showed higher 7D12-R2 levels than 7D12 (Figure 3a). In general, $\gamma$-counting and SPECT data were strongly correlated (Figure S3), showing that a smaller tracer results in higher tumor uptake with lower background.

(a)
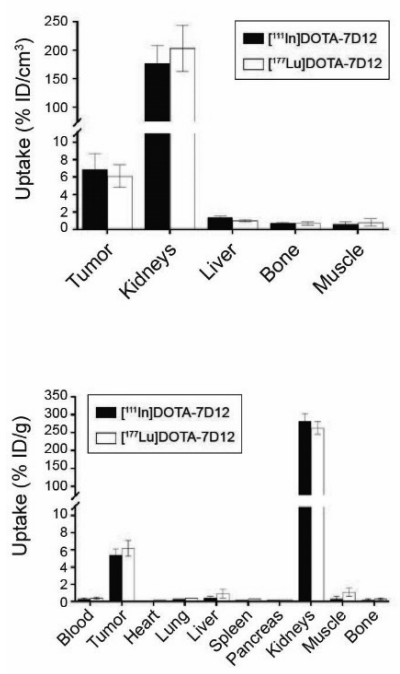

(b)

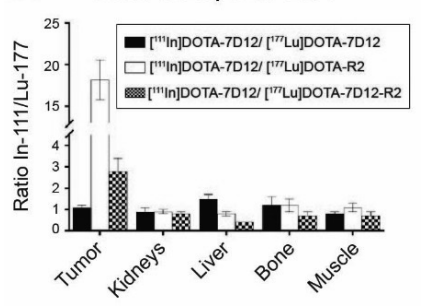

Dual-isotope SPECT
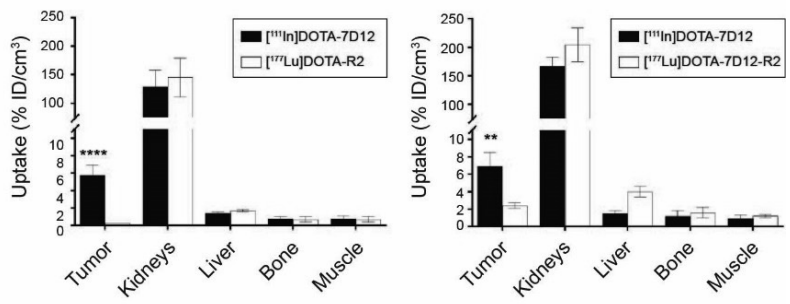

Y-counting
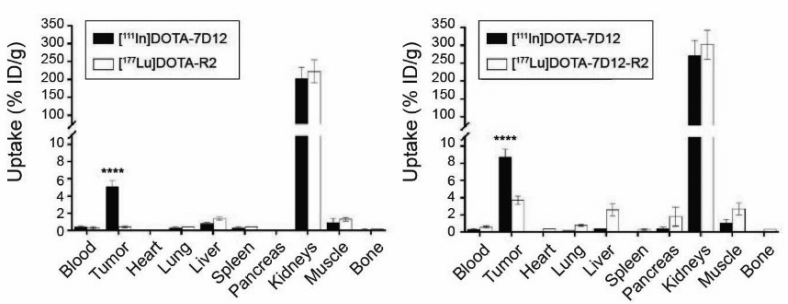

Figure 3 Biodistribution of NB couples. (a) Biodistribution data from SPECT imaging and $\gamma$ counting $1 \mathrm{~h}$ after injection of $\left[{ }^{111} \mathrm{In}\right]$ DOTA-7D12/[ $\left[{ }^{177} \mathrm{Lu}\right]$ DOTA-7D12, $\left[{ }^{111} \mathrm{In}\right]$ DOTA7D12/[ $\left[{ }^{177} \mathrm{Lu}\right]$ DOTA-R2, or $\left[{ }^{111} \mathrm{In}\right]$ DOTA-7D12/[ $\left.{ }^{177} \mathrm{Lu}\right]$ DOTA-7D12-R2 in tumor-bearing mice $(n=4)$. Statistical significance of the observed differences between the tumor uptake of each NB was determined (mean $\pm \mathrm{SD}$ ). ${ }^{* *} \mathrm{p} \leq 0.01$ and $* * * * \mathrm{p} \leq 0.0001$. (b) Ratios $\left[{ }^{111} \mathrm{In}\right] \mathrm{DOTA}$ $\mathrm{NB} /\left[{ }^{177} \mathrm{Lu}\right] \mathrm{DOTA}-\mathrm{NB}$ calculated from SPECT and $\gamma$-counting biodistribution data (mean $\pm \mathrm{SD}$ ). 


\section{Effect of Binding Specificity and of Tracer Molecular Size on Distribution Through Tumors}

Autoradiographic analysis of tumor sections indicated high accumulation of the In-111 and Lu-177 signal in the highly perfused rim of the tumors after injection of [ $\left.{ }^{111} \mathrm{In}\right] \mathrm{DOTA}$ 7D12/[ $\left.{ }^{177} \mathrm{Lu}\right]$ DOTA-7D12 or $\left[{ }^{111} \mathrm{In}\right] \mathrm{DOTA}-7 \mathrm{D} 12 /\left[{ }^{177} \mathrm{Lu}\right] \mathrm{DOTA}-7 \mathrm{D} 12-\mathrm{R} 2$ (Figure 4a). Importantly, signal corresponding to 7D12 was more evenly distributed throughout the tumor. Quantification of autoradiographic images from all mice confirmed that the tumor uptake of 7D12 was similar in all animals, regardless of the co-injection with other NBs or the choice of the radionuclide (Figure 4b). Moreover, the presence of R2 in the tumor was negligible, whereas the signal from 7D12 was always higher than 7D12-R2.
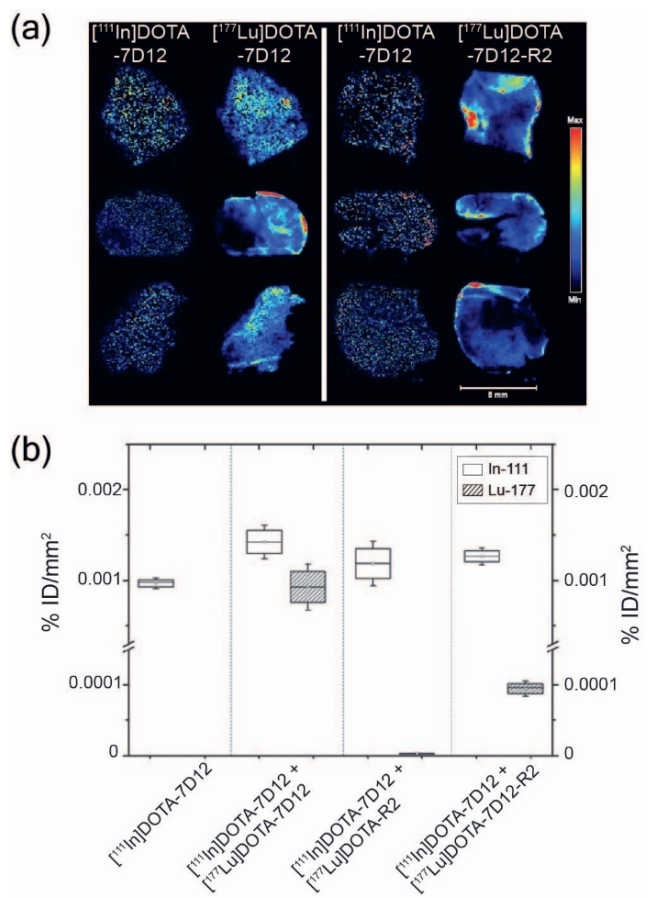

Figure 4 Ex vivo autoradiography of tumor tissue and signal quantification. (a) Tumor-bearing mice were co-injected with $\left[{ }^{111} \mathrm{In}\right]$ DOTA-7D $12 /\left[{ }^{177} \mathrm{Lu}\right]$ DOTA-7D12 or $\left[{ }^{111}\right.$ In $]$ DOTA$7 \mathrm{D} 12 /\left[{ }^{177} \mathrm{Lu}\right] \mathrm{DOTA}-7 \mathrm{D} 12-\mathrm{R} 2$ and tumor sections were imaged based on decay rates. Representative tumor sections are shown. (b) Radioactivity quantification from autoradiographic images of tumor sections from mice injected with $\left[{ }^{111} \mathrm{In}\right] \mathrm{DOTA}-7 \mathrm{D} 12$, [ $\left.{ }^{111} \mathrm{In}\right] \mathrm{DOTA}$ 7D12/ $\left[{ }^{177}\right.$ Lu $]$ DOTA-7D12, $\quad\left[{ }^{111}\right.$ In]DOTA-7D12/ $\left[{ }^{177}\right.$ Lu $] D O T A-R 2, \quad$ or $\quad\left[{ }^{111}\right.$ In $]$ DOTA$7 \mathrm{D} 12 /\left[{ }^{177} \mathrm{Lu}\right] \mathrm{DOTA}-7 \mathrm{D} 12-\mathrm{R} 2$. Box plots display the range between first and third quartile, median and full data range (whiskers).

\section{Discussion}

Many factors influence tumor uptake and distribution of tracers such as NBs, thus making it challenging to determine the most optimal tracer format for molecular imaging of solid tumors. In the present study, we have investigated the combination of imaging of in vitro tumor spheroids, in vivo dual-isotope SPECT, and ex vivo autoradiographic analysis of 
tumor sections, to efficiently assess the tumor uptake and distribution of different NB formats, while using a reduced number of animals. Importantly, the three approaches yielded similar results. The similarity in the results obtained with the 3D spheroid model and the in vivo model suggests that the spheroids could predict the in vivo tumor distribution of the tracers. Although further studies with different targets and models are needed, 3D spheroids models have the potential to reduce the number of in vivo studies needed to compare different formats of tracers. Dual-isotope SPECT imaging was successfully confirmed here as a valuable technique for small animal NB imaging. Tumor uptake and biodistribution of each co-injected NB were quantified, with a strong linear relation with ex vivo $\gamma$-counting. The imaging and quantification of tracer couple within the same animal over time eliminates inter-animal differences, considerably reducing time and number of animals needed. Furthermore, quantitative autoradiographic analysis of tumor sections allowed to more precisely compare the intratumoral distribution of the tracers.

In this study, we assessed the tumor accumulation of the irrelevant NB R2 and the EGFR-targeted NB 7D12. R2 was not retained in tumors in vivo, in agreement with the absence of accumulation into spheroids. On the contrary, 7D12 accumulated in spheroids and showed a 19-times higher tumor uptake than R2, when co-injected in mice. The considerably higher tumor uptake of a specific NB in comparison with an irrelevant NB is in agreement with previous studies evaluating single NBs with optical imaging [17] and single-isotope SPECT $[19,20]$. We also assessed the behavior of tumor targeting NBs with different molecular size, but similar binding affinity (i.e., 7D12 and 7D12-R2). Our in vitro data showed that the monomeric NB 7D12 was able to more rapidly and more homogeneously distribute throughout spheroids over time, compared with the dimeric NB 7D12-R2. In addition, our in vitro data reinforces the notion that NBs penetrate tumors faster and deeper than mAbs, as documented in other studies [7,17,21]. Although tumor spheroids resemble many of the characteristics of an in vivo tumor, several aspects are not reproduced in this model, e.g., capillary extravasation and systemic clearance. Spheroids experiments showed that the accumulation of NBs into spheroids increased during the first 3-4 h, while the SPECT data showed no significant differences in tumor uptake of NBs between 1 and $3 \mathrm{~h}$ post-injection. This is probably caused by the rapid clearance of NBs via the kidneys (in vivo half-life $\sim 90 \mathrm{~min}$ [22]), which is not reproduced in the spheroid model. Importantly, 7D12 accumulated in tumors to a degree of 5-8\% $\mathrm{ID} / \mathrm{cm} 3$, which was similar to the reported $4.62 \% \mathrm{ID} / \mathrm{cm} 3$ for this NB using singe-isotope SPECT [18]. Remarkably, the increase of $16 \mathrm{kDa}$ for 7D12-R2 caused a 2.5-times reduction in tumor uptake. This result underlines the effect of molecular size of the tracer on tumor uptake and is consistent with other studies evaluating similar NB formats with single-isotope SPECT $[19,20]$, thus supporting the reliability of the methods employed in the present study. Lastly, the autoradiographic images of tumor sections revealed that the dimeric NB had a limited penetration into the tumor in comparison to the monomer, which was also observed in vitro as a delayed penetration into spheroids.

Essential for imaging is the contrast between tumor and control tissues. In general, SPECT resulted in slightly lower uptake values in organs $(\sim 10 \%)$ than $\gamma$-counting, which is most likely due to ineffective crosstalk removal and partial volume effects at high activities [23]. This effect was more pronounced in the kidneys (approximately $30 \%$ of the injected dose was observed in each kidney), but values in kidneys were comparable with those from single-isotope imaging of EGFR-targeted NBs [18,24]. We also observed a retention of the dimeric 7D12-R2 in blood and other organs, which has been reported for other dimeric NBs as well [19]. 
Overall, the consistency of our results with published data confirms the value of the combination of techniques used here and demonstrates that this combination of techniques is useful to assess the tumor uptake and distribution of different (NB-based) tracers. Including imaging of 3D spheroids models in the in vitro characterization of new tracers, could lead to earlier identification of (un)promising candidates. The use of dualisotope SPECT imaging in the subsequent preclinical studies could considerably reduce the number of animals needed. Lastly, autoradiographic analysis of the tumors could provide additional data on the distribution of tracers throughout tumors. In our case, the use of only four mice per group (per couple of NBs to investigate) led to unambiguous data because of the elimination of inter-animal variations. In contrast, standard biodistributions studies would generally require six mice per tracer and per time point $[14,19]$.

\section{Conclusion}

A combination of in vitro experiments involving tumor spheroids and in vivo experiments with dual-isotope SPECT imaging and subsequent ex vivo autoradiography of tumors provided an accurate longitudinal study of different NB tracers, using a reduced number of animals. This combination of techniques can accelerate the design of novel targeting compounds, which could ultimately contribute to their progress to the clinic, for molecular imaging as well as other applications.

\section{References}

1. Khemthongcharoen, N.; Jolivot, R.; Rattanavarin, S.; Piyawattanametha, W. Advances in imaging probes and optical microendoscopic imaging techniques for early in vivo cancer assessment. $A d v$. Drug Deliv. Rev. 2014, 74, 53-74.

2. Chakravarty, R.; Goel, S.; Cai, W. Nanobody: The "Magic Bullet" for Molecular Imaging? Theranostics 2014, 4, 386-398.

3. Mishani, E.; Hagooly, A. Strategies for molecular imaging of epidermal growth factor receptor tyrosine kinase in cancer. J. Nucl. Med. 2009, 50, 1199-1202.

4. Jain, R.K. Physiological barriers to delivery of monoclonal antibodies and other macromolecules in tumors. Cancer Res. 1990, 50 .

5. Xenaki, K.T.; Oliveira, S.; van Bergen En Henegouwen, P.M.P. Antibody or antibody fragments: implications for molecular imaging and targeted therapy of solid tumors. Front Immunol. 2017, 8 , 1287.

6. Oliveira, S.; Heukers, R.; Sornkom, J.; Kok, R.J.; van Bergen En Henegouwen, P.M.P. Targeting tumors with nanobodies for cancer imaging and therapy. J. Control. Release 2013, 172, 607-17.

7. Kijanka, M.; Warnders, F.J.; El Khattabi, M.; Lub-De Hooge, M.; Van Dam, G.M.; Ntziachristos, V.; De Vries, L.; Oliveira, S.; Van Bergen En Henegouwen, P.M.P. Rapid optical imaging of human breast tumour xenografts using anti-HER2 VHHs site-directly conjugated to IRDye $800 \mathrm{CW}$ for image-guided surgery. Eur. J. Nucl. Med. Mol. Imaging 2013, 40, 1718-1729.

8. Xavier, C.; Blykers, A.; Vaneycken, I.; D’Huyvetter, M.; Heemskerk, J.; Lahoutte, T.; Devoogdt, N.; Caveliers, V. (18)F-nanobody for PET imaging of HER2 overexpressing tumors. Nucl. Med. Biol. 2016, 43, 247-252.

9. Keyaerts, M.; Xavier, C.; Heemskerk, J.; Devoogdt, N.; Everaert, H.; Ackaert, C.; Vanhoeij, M.; Duhoux, F.P.; Gevaert, T.; Simon, P.; et al. Phase I study of 68Ga-HER2-nanobody for PET/CT assessment of HER2 expression in breast carcinoma. J. Nucl. Med. 2015, 57, $27-33$.

10. Wållberg, H.; Ståhl, S. Design and evaluation of radiolabeled tracers for tumor imaging. Biotechnol. Appl. Biochem. 2013, 60, 365-383. 
11. Antoni, D.; Burckel, H.; Josset, E.; Noel, G. Three-Dimensional Cell Culture: A Breakthrough in Vivo. Int. J. Mol. Sci. 2015, 16, 5517-5527.

12. Blaire, T.; Bailliez, A.; Ben Bouallègue, F.; Bellevre, D.; Agostini, D.; Manrique, A. Determination of the heart-to-mediastinum ratio of 123 I-MIBG uptake using dual-isotope ( 123 I-MIBG/ 99m Tc-tetrofosmin) multi-pinhole CZT SPECT in patients with heart failure. J. Nucl. Med. 2017.

13. Shiga, T.; Suzuki, A.; Sakurai, K.; Kurita, T. Dual Isotope SPECT Study With Epilepsy Patients Using Semiconductor SPECT System. 2017, 42, 663-668.

14. Hijnen, N.M.; de Vries, A.; Nicolay, K.; Grüll, H. Dual-isotope $111 \mathrm{In} / 177 \mathrm{Lu}$ SPECT imaging as a tool in molecular imaging tracer design. Contrast Media Mol. Imaging 2012, 7, 214-22.

15. Frenken, L.G.; van der Linden, R.H.; Hermans, P.W.; Bos, J.W.; Ruuls, R.C.; de Geus, B.; Verrips, C.T. Isolation of antigen specific llama VHH antibody fragments and their high level secretion by Saccharomyces cerevisiae. J. Biotechnol. 2000, 78, 11-21.

16. Van Driel, P.B.A.A.; Boonstra, M.C.; Slooter, M.D.; Heukers, R.; Stammes, M.A.; Snoeks, T.J.A.; De Bruijn, H.S.; Van Diest, P.J.; Vahrmeijer, A.L.; Van Bergen En Henegouwen, P.M.P.; et al. EGFR targeted nanobody-photosensitizer conjugates for photodynamic therapy in a pre-clinical model of head and neck cancer. J. Control. Release 2016, 229, 93-105.

17. Oliveira, S.; Van Dongen, G. a M.S.; Stigter-Van Walsum, M.; Roovers, R.C.; Stam, J.C.; Mali, W.; Van Diest, P.J.; Van Bergen En Henegouwen, P.M.P. Rapid visualization of human tumor xenografts through optical imaging with a near-infrared fluorescent anti-epidermal growth factor receptor nanobody. Mol. Imaging 2012, 11, 33-46.

18. Gainkam, L.O.T.; Huang, L.; Caveliers, V.; Keyaerts, M.; Hernot, S.; Vaneycken, I.; Vanhove, C.; Revets, H.; De Baetselier, P.; Lahoutte, T. Comparison of the Biodistribution and Tumor Targeting of Two 99mTc-Labeled Anti-EGFR Nanobodies in Mice, Using Pinhole SPECT/Micro-CT. J. Nucl. Med. 2008, 49, 788-795.

19. Movahedi, K.; Schoonooghe, S.; Laoui, D.; Houbracken, I.; Waelput, W.; Breckpot, K.; Bouwens, L.; Lahoutte, T.; De Baetselier, P.; Raes, G.; et al. Nanobody-based targeting of the macrophage mannose receptor for effective in vivo imaging of tumor-associated macrophages. Cancer Res. 2012, 72, 4165-4177.

20. Krasniqi, A.; Bialkowska, M.; Xavier, C.; Jeught, K. Van Der; Muyldermans, S.; Devoogdt, N.; Huyvetter, M.D. Pharmacokinetics of radiolabeled dimeric sdAbs constructs targeting human CD20. N. Biotechnol. 2018, 45, 69-79.

21. Bannas, P.; Lenz, A.; Kunick, V.; Well, L.; Fumey, W.; Rissiek, B.; Haag, F.; Schmid, J.; Schütze, K.; Eichhoff, A.; et al. Molecular imaging of tumors with nanobodies and antibodies: Timing and dosage are crucial factors for improved in vivo detection. Contrast Media Mol. Imaging 2015, 10, 367-378.

22. Roovers, R.C.; Laeremans, T.; Huang, L.; De Taeye, S.; Verkleij, A.J.; Revets, H.; De Haard, H.J.; van Bergen En Henegouwen, P.M.P. Efficient inhibition of EGFR signalling and of tumour growth by antagonistic anti-EGFR Nanobodies. Cancer Immunol. Immunother. 2007, 56, 303-317.

23. Pretorius, P.H.; King, M.A. Diminishing the impact of the partial volume effect in cardiac SPECT perfusion imaging. Med. Phys. 2008, 36, 105-115.

24. Gainkam, L.O.T.; Keyaerts, M.; Caveliers, V.; Devoogdt, N.; Vanhove, C.; Van Grunsven, L.; Muyldermans, S.; Lahoutte, T. Correlation between epidermal growth factor receptor-specific nanobody uptake and tumor burden: A tool for noninvasive monitoring of tumor response to therapy. Mol. Imaging Biol. 2011, 13, 940-948. 


\section{Supplementary Material}
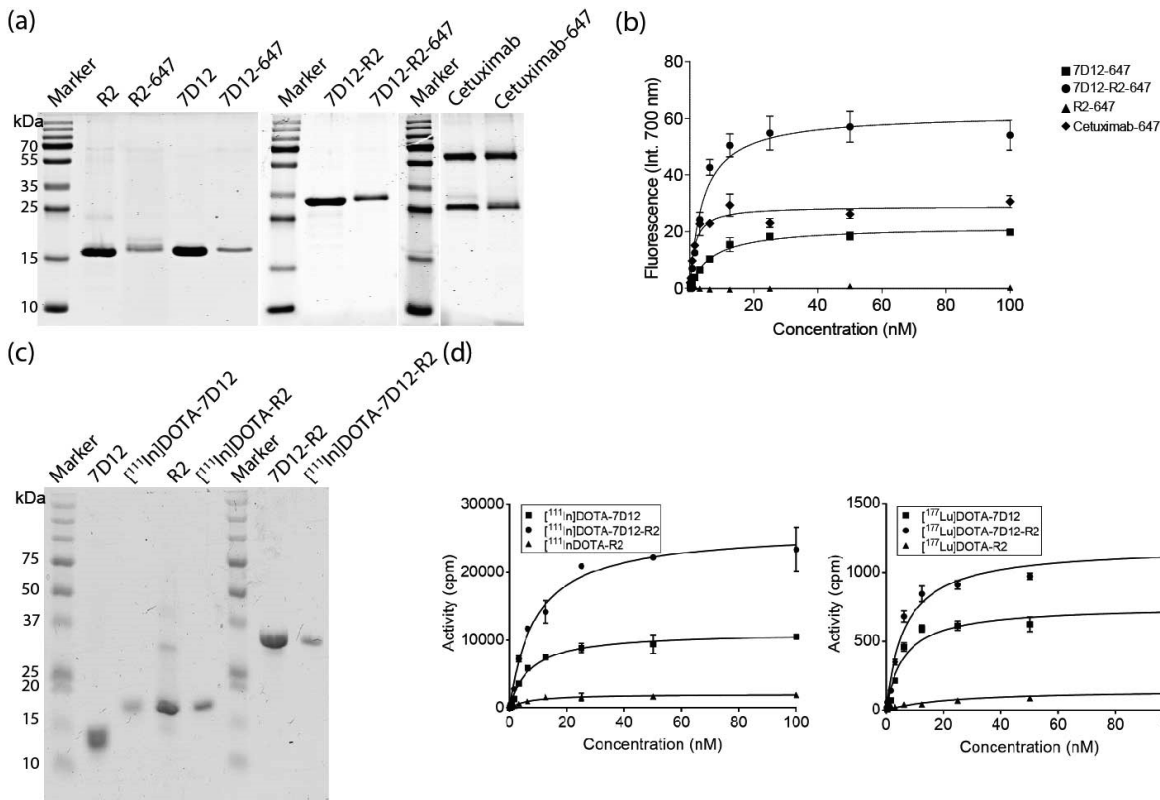

(d)

(e)
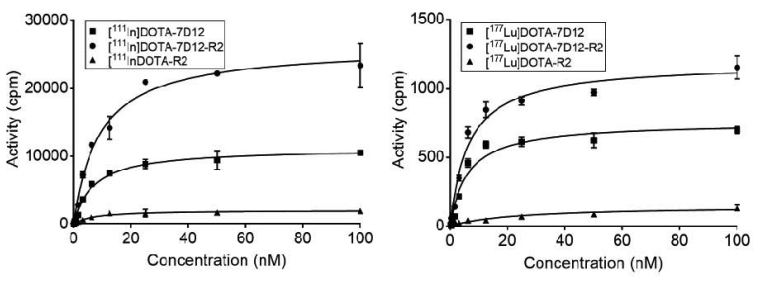

\begin{tabular}{|c|c|c|c|c|c|c|c|c|c|}
\hline \multicolumn{10}{|c|}{ Apparent affinity (nM) } \\
\hline 7D12 & 7D12-647 & $\begin{array}{c}{[" 11 \text { In]DOTA }} \\
-7 D 12\end{array}$ & $\begin{array}{c}{\left[{ }^{177} \angle \text { LUDOTA }\right.} \\
-7 \mathrm{D} 12\end{array}$ & 7D12-R2 & 7D12-R2-647 & 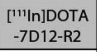 & 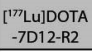 & Cetuximab & \begin{tabular}{|c|} 
Cetuximab \\
-647 \\
\end{tabular} \\
\hline $1.6 \pm 0.2$ & $6.5 \pm 0.4$ & $5.2 \pm 1.2$ & $5.3 \pm 0.6$ & $2.7 \pm 0.3$ & $9.6 \pm 5.5$ & $5.4 \pm 1.2$ & $6.3 \pm 3.1$ & $0.05 \pm 0.01$ & $1.3 \pm 0.2$ \\
\hline
\end{tabular}

Figure S1 Characterization of labeled proteins. (a) Alexa 647-conjugated and unconjugated proteins analyzed by $15 \%$ SDS-PAGE gel stained with PageBlue Protein staining. (b) Apparent affinities of the Alexa 647-labeled proteins were determined via a binding assay with A431 cells using a concentration range $(0.2-100 \mathrm{nM})$ of each conjugate. Bound conjugate was detected by scanning at $700 \mathrm{~nm}$. (c) SDS-PAGE of the non-labeled and indium-111 labeled NBs stained with Coomassie brilliant blue. (d) In vitro assessment of the apparent affinities of indium-111 labeled NBs and lutetium-177 labeled NBs on A431 cells using a concentration range $(0.2-100 \mathrm{nM})$ of each radiolabeled protein. Bound fraction was detected with a $\gamma$-counter (mean \pm SD). (e) Apparent affinities (KD values) of the unlabeled [7,8] and labeled proteins. 
(a)

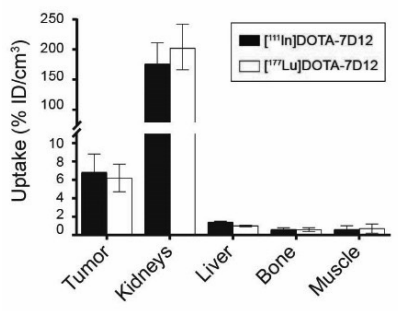

Dual-isotope SPECT
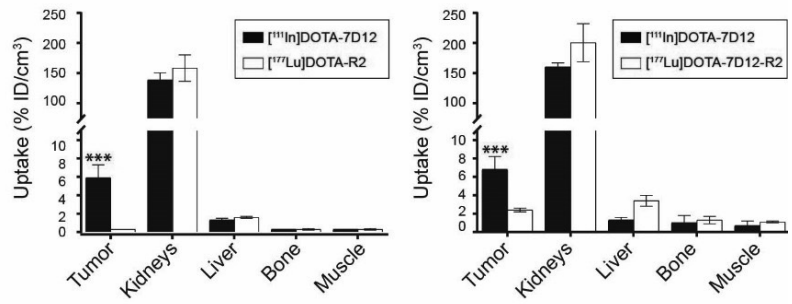

Y-counting
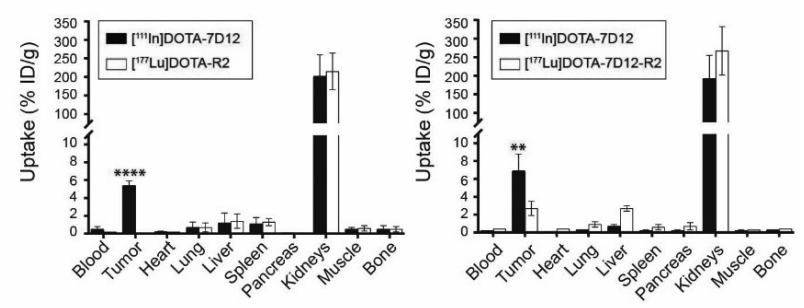

(b)

Dual-isotope SPECT
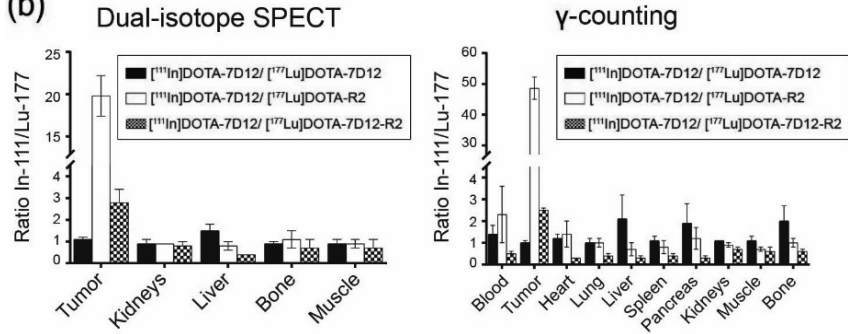

Figure S2 Biodistribution of NB couples. (a) Biodistribution results from SPECT imaging and $\gamma$ counting after $3 \mathrm{hrs}$ co-injection of $\left[{ }^{111}\right.$ In]DOTA-7D12/ $\left[{ }^{177} \mathrm{Lu}\right] \mathrm{DOTA}-7 \mathrm{D} 12,\left[{ }^{111}\right.$ In]DOTA7D12/[ $\left.{ }^{177} \mathrm{Lu}\right]$ DOTA-R2 or $\left[{ }^{111} \mathrm{In}\right] \mathrm{DOTA}-7 \mathrm{D} 12 /\left[{ }^{177} \mathrm{Lu}\right] \mathrm{DOTA}-7 \mathrm{D} 12-\mathrm{R} 2$. Statistical significance of the observed differences between the tumor uptake of each NB were determined. $* * p \leq 0.01$, $* * * \mathrm{p} \leq 0.001$, and $* * * * \mathrm{p} \leq 0.0001$. (b) Ratios $\left[{ }^{111} \mathrm{In}\right] \mathrm{DOTA}-\mathrm{NB} /\left[{ }^{177} \mathrm{Lu}\right] \mathrm{DOTA}-\mathrm{NB} 3$ hrs postinjection (mean $\pm \mathrm{SD})$. 


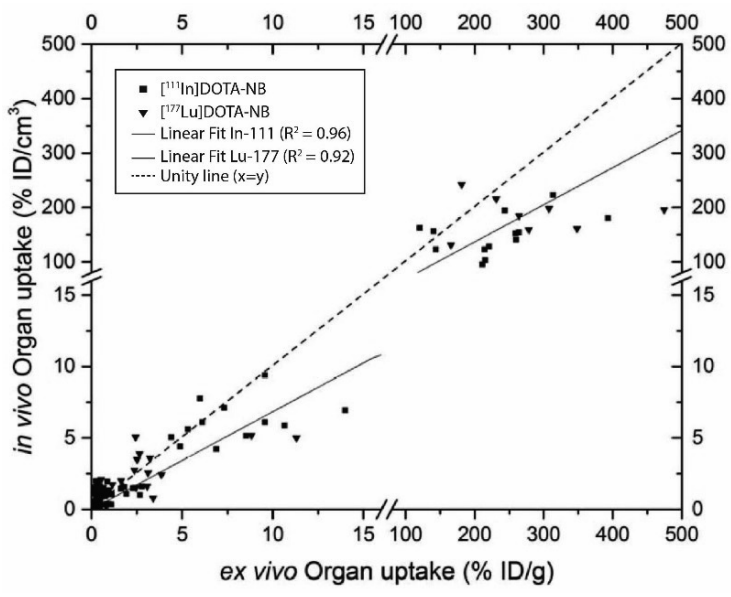

Figure S3 Correlation of SPECT (y-axis) and $\gamma$-counter (x-axis) results. Data from tumor bearing mice injected with 7D12, 7D12/R2, 7D12/7D12, and 7D12/7D12-R2 were used. Plotting and fitting were done in Origin 9. 



\section{CHAPTER 3}

\section{Patient-derived head and neck cancer organoids recapitulate EGFR expression levels of respective tissues and are responsive to EGFR-targeted photodynamic therapy}

Else Driehuis ${ }^{1, *}$, Sacha Spelier ${ }^{1,2, *}$, Irati Beltrán Hernández ${ }^{2,3}$, Remco de Bree $^{4}$, Stefan M. Willems ${ }^{5}$, Hans Clevers ${ }^{1,6}$ and Sabrina Oliveira ${ }^{2,3}$

\footnotetext{
${ }^{1}$ Oncode Institute, Hubrecht Institute, Royal Netherlands Academy of Arts and Sciences (KNAW) and University Medical Center Utrecht, Utrecht, the Netherlands

${ }^{2}$ Cell Biology, Neurobiology and Biophysics, Department of Biology, Faculty of Science, Utrecht University, Utrecht, the Netherlands

${ }^{3}$ Pharmaceutics, Department of Pharmaceutical Sciences, Faculty of Science, Utrecht University, Utrecht, the Netherlands

${ }^{4}$ Department of Head and Neck Surgical Oncology, University Medical Center Utrecht, Utrecht, the Netherlands

${ }^{5}$ Department of Pathology, University Medical Center Utrecht, Utrecht, the Netherlands

${ }^{6}$ Princess Maxima Center, Utrecht, the Netherlands

* These authors contributed equally to this work
} 


\begin{abstract}
Patients diagnosed with head and neck squamous cell carcinoma (HNSCC) are currently treated with surgery and/or radio- and chemotherapy. Despite these therapeutic interventions, $40 \%$ of patients relapse, urging the need for more effective therapies. In photodynamic therapy (PDT), a light-activated photosensitizer produces reactive oxygen species that ultimately lead to cell death. Targeted PDT, using a photosensitizer conjugated to tumor-targeting molecules, has been explored as a more selective cancer therapy. Organoids are self-organizing three-dimensional structures that can be grown from both normal and tumor patient-material and have recently shown translational potential. Here, we explore the potential of a recently described HNSCC-organoid model to evaluate Epidermal Growth Factor Receptor (EGFR)-targeted PDT, through either antibody- or nanobody-photosensitizer conjugates. We find that EGFR expression levels differ between organoids derived from different donors, and recapitulate EGFR expression levels of patient material. EGFR expression levels were found to correlate with the response to EGFR-targeted PDT. Importantly, organoids grown from surrounding normal tissues showed lower EGFR expression levels than their tumor counterparts, and were not affected by the treatment. In general, nanobody-targeted PDT was more effective than antibody-targeted PDT. Taken together, patient-derived HNSCC organoids are a useful 3D model for testing in vitro targeted PDT.
\end{abstract}




\section{Introduction}

Head and neck squamous cell carcinoma (HNSCC) is a collective term used for tumors of the stratified epithelium that lines the oral cavity, pharynx, and larynx [1]. Depending on anatomical location, tumor stage; and patient age, fitness, and comorbidities, the treatment of HNSCC can consist of surgery, radiotherapy, and chemotherapy (either alone or in combination). Early stage (stage I/II) HNSCC is usually treated with surgery or radiotherapy alone, and has a favorable prognosis. However, over $60 \%$ of patients present advanced stage disease (stage III/IV) at the time of diagnosis [2]. For these patients, treatment with curative intent consists of surgery with adjuvant radiotherapy, either alone or combined with chemotherapy, or chemoradiation with surgery in reserve for eventual salvage treatment. While surgery and radiotherapy are applied locally, these therapies may compromise important functions such as mastication and swallowing, thereby significantly decreasing the patients' quality of life. Commonly used chemotherapeutics cisplatin and carboplatin serve as radiosensitizers, and are therefore given concurrently with radiotherapy. Although effective in a subset of patients, these treatments bring about severe side-effects [3]. Epidermal Growth Factor Receptor (EGFR) protein is overexpressed in $50 \%-90 \%$ of $\mathrm{HNSCC}$, from which $15 \%$ carries EGFR gene amplification [4]. Accordingly, the EGFR-targeting antibody cetuximab was introduced as an alternative treatment strategy for patients ineligible for cisplatin or carboplatin treatment [4-7]. Despite these interventions, relapse rates remain around 50\% for advanced stage HNSCC [8].

The limited efficacy and harsh side-effects of current treatments emphasize the need for more selective treatment strategies for HNSCC. As surgery is a main component of HNSCC treatment and tumors are often accessible, photodynamic therapy (PDT), or even the more recently explored targeted PDT, could enable such a targeted and local effect, and thereby serve as a potential therapy to treat localized HNSCC. Conventional PDT starts with the administration of a photosensitizer (PS), which is excited by locally applied light after 2-4 days. The activated PS subsequently converts oxygen to reactive oxygen species (ROS) that can damage DNA, proteins, and lipids, ultimately resulting in cell death [9-11]. In addition, PDT has been shown to contribute to tumor vasculature destruction and activation of the immune system [12]. Side-effects of conventional PDT (using hydrophobic PS) are common, including damage to normal surrounding tissues and skin phototoxicity. By conjugation of the PS to a tumor-targeting molecule such as an antibody, PDT can be made more tumor-specific. Although encouraging results have been obtained over the years, targeted PDT has only recently entered clinical testing [13]. The development of water-soluble PSs (such as the silicon phthalocyanine derivative IRDye700DX) has contributed to this, rendering antibody-PS conjugates more stable and eliminating the need for long spacers between antibody and PS [14]. Targeted PDT with cetuximab-IRDye700DX conjugates is currently being tested in patients diagnosed with advanced stage HNSCC (NCT02422979) [15]. The first results of this trial indicate that patients responded well to this therapy, while experiencing limited side-effects.

We have recently introduced nanobody-targeted PDT as an alternative to antibodytargeted PDT $[16,17]$. Nanobodies are the variable domain of a subset of antibodies that consist of heavy chains only, which are found in only a small subset of animals, including camelids [18]. Nanobodies are approximately ten times smaller than conventional antibodies, allowing for both a quicker and a more homogenous tissue penetration, as well as a faster systemic clearance when left unbound [16]. Compared to antibody-PS conjugates, the use of nanobody-PS conjugates is expected to require shorter time intervals between administration and light application ( 1 or $2 \mathrm{~h}$, instead of day(s)), lead 
to more extensive tumor damage, and result in less skin photosensitivity. Using cell lines and xenografts, we have previously shown that nanobody-targeted PDT is selective for cells with high EGFR expression in vitro [16], and induces extensive tumor damage in an in vivo HNSCC model [17]. Although these results are promising, it is unclear whether EGFR expression levels of the cell lines used represent the expression of EGFR in human samples [19]. As such, it remains uncertain if findings reported thus far can be translated to the clinic.

Organoids are three-dimensional self-organizing structures that can be grown from stem cells, and recapitulate the organization, histological features, and to some extent, functional characteristics of epithelial tissues [20]. Organoids can be established with high efficiency from patient-derived material, such as surgical resections or tumor biopsies, and allow for the expansion of patient-derived tumor and normal cells in vitro. We recently developed an organoid model for HNSCC, which recapitulates morphological and genetic characteristics of this tumor type, and was found to be eligible for in vitro drug testing [21]. These 3D structures have been established from tumor and tumor-adjacent wildtype tissue, consist of proliferating squamous epithelial cells, and can be maintained in culture for over a year. Transplantation of the tumor-derived structures resulted in tumor-formation in mice, showing that these 'mini-tumors' retain their tumorigenic potential in vitro. Tumor-status of the organoids was confirmed using DNA sequencing (which included targeted sequencing of EGFR), and organoids were further characterized using RNA sequencing and functional drug screens. When exposed to a range of therapeutic agents, including the EGFR-targeting antibody cetuximab, we observed variable responses between organoids derived from different donors [21]. Normal organoids consisting of wildtype cells (status confirmed by DNA sequencing), on the other hand, did not result in tumor formation when transplanted.

In this study, we aim to characterize EGFR expression levels on these previously described HNSCC-derived tumor organoids [21], and compare the expression level to that of cell lines used in our previous studies. Subsequently, we investigate EGFRtargeted PDT in this 3D patient-derived model. Although, untargeted PDT has previously been tested in 3D cell cultures [22-25], to our knowledge, this is the first report of in vitro PDT in a 3D patient-derived model. Our results point to the relevance of employing patient-derived material, compared to an established cell line, for testing of targeted therapies, and support that these organoids are a useful 3D model for testing these.

\section{Materials and Methods}

\section{Human Material for Organoid Cultures}

The collection of patient data and tissue for the generation and distribution of organoids was performed according to the guidelines of the European Network of Research Ethics Committees (EUREC) following European and national law. The Biobank Research Ethics Committee of the UMC Utrecht (TCBio) approved the biobanking protocol: 12093 HUB-Cancer according to the UMCU Biobanking Regulation. All donors participating in this study signed informed consent forms and can withdraw their consent at any time. Available organoids will be catalogued at www.hub4organoids.eu and can be requested atinfo@hub4organoids.eu. 


\section{Tissue Processing}

Patient material was collected from pathology material in Advanced DMEM/F12 (Life Technologies), supplemented with 1× GlutaMAX (adDMEM/F12; Life Technologies), Penicillin-streptomycin (Life Technologies) and $10 \mathrm{mM}$ HEPES (Life Technologies). This medium was named $+/+/+$. Patient material was collected in $+/+/+$ containing 100 $\mu \mathrm{g} / \mathrm{mL}$ Primocin (Invivogen). For normal tissue samples, excess fat or muscle tissue was removed to enrich for epithelial cells and tissue was cut into small fragments. Random pieces of approximately $5 \mathrm{~mm}^{3}$ were stored at $-20{ }^{\circ} \mathrm{C}$ for DNA isolation. Two to four tissue pieces were fixed in formalin for histopathological analysis and immunohistochemistry, and the remainder was processed for organoid derivation. Fragments were incubated at $37{ }^{\circ} \mathrm{C}$ in $0.125 \%$ Trypsin (Sigma-Aldrich) in $+/+/+$ until digested. Every $10 \mathrm{~min}$, the tissue suspension was sheared using $1 \mathrm{~mL}$ pipette. Incubation was performed for a maximum of $60 \mathrm{~min}$. When complete, suspension was diluted with $+/+/+$ and strained over a $100 \mu \mathrm{m}$ EasyStrainer filter (Greiner Bio-one), followed by centrifugation at $300 \times \mathrm{g}$. The resulting pellet was resuspended in ice-cold $70 \% 10 \mathrm{mg} / \mathrm{mL}$ cold Cultrex growth factor reduced Basement Membrane Extract (BME) type 2 (Trevigen) in organoid medium, which serves as a extracellular matrix mimetic in the organoid cultures. Approximately 10,000 cells were resuspended per $40 \mu \mathrm{L}$ of BME. Droplets of approximately $10 \mu \mathrm{L}$ were plated on the bottom of pre-heated suspension culture plates (Greiner Bio-one). After plating, plates were inverted and put at $37^{\circ} \mathrm{C}$ for $30 \mathrm{~min}$ to let the BME solidify. Subsequently, prewarmed organoid medium was added to the plate. For the first week, $10 \mu \mathrm{M}$ Rho-associated kinase (ROCK) inhibitor Y-27632 (Abmole Bioscience) was added to the medium to aid outgrowth of organoids.

\section{Organoid Culture}

The organoid medium consisted of $+/+/+$, supplemented with $1 \times$ B27 supplement (Life Technologies), 1,25 mM N-acetyl-L-cysteine (Sigma-Aldrich), $10 \mathrm{mM}$ Nicotinamide (Sigma-Aldrich), $50 \mathrm{ng} / \mathrm{mL}$ human EGF (PeproTech), $500 \mathrm{nM} \mathrm{A83-01,} 10 \mathrm{ng} / \mathrm{mL}$ human FGF10 (PeproTech), $5 \mathrm{ng} / \mathrm{mL}$ human FGF2 (PeproTech), $1 \mu \mathrm{M}$ Prostaglandin E2 (Tocris Bioscience), $3 \mu \mathrm{M}$ CHIR 99021 (Sigma-Aldrich), $1 \mu \mathrm{M}$ Forskolin (Bio-Techne), 4\% $(\mathrm{v} / \mathrm{v})$ RSPO, and $4 \%(\mathrm{v} / \mathrm{v})$ Noggin (both produced via the r-PEX protein expression platform at U-Protein Express BV). Organoids were split between 7 and 14 days after initial plating. For passaging, organoids were collected from the plate by disrupting the BME droplets with a P1000 pipette and washed in $10 \mathrm{~mL}+/+/+$. The pellet was resuspended in $1 \mathrm{~mL}$ of TrypLE Express (Life Technologies) for incubation at $37^{\circ} \mathrm{C}$. Digestion was closely monitored and suspension was pipetted up and down every $5 \mathrm{~min}$ to aid disruption of the organoids. TrypLE digestion was stopped when organoids were disrupted into single cells by adding $10 \mathrm{~mL}+/+/+$. Cells were resuspended in ice-cold $70 \%$ BME in organoid medium and plated at suitable ratios $(1: 5$ to $1: 20)$ to allow efficient outgrowth of new organoids. Directly after splitting, $10 \mu \mathrm{M}$ Y-27632 was added to aid outgrowth of organoids from single cells. Medium was changed every 2-3 days and organoids were split once every 1-2 weeks.

\section{Cell Line Culture}

Human vulvar squamous cell carcinoma A431 (CRL-1555), human cervical carcinoma cell line HeLa (CCL-2), and human embryonal kidney cell line HEK293T (CRL-3216) were purchased from the American Type Culture Collection (ATCC). Human head and neck squamous cell carcinoma cell line UM-SCC-14C (14C) was kindly provided by Prof. Dr. T.E. Carey (University of Michigan, USA). All cell lines were cultured in 
DMEM (Life Technologies) supplemented with $10 \%$ fetal calf serum, and $100 \mu \mathrm{g} / \mathrm{mL}$ penicillin/streptomycin (Life Technologies). Cells were cultured at $37{ }^{\circ} \mathrm{C}, 5 \% \mathrm{CO} 2$ in a humidified atmosphere, and split with TrypLE Express (Life Technologies) for $10 \mathrm{~min}$ at $37^{\circ} \mathrm{C}$ once a week.

\section{RNA Collection}

Organoids were cultured as normal. For quantification of EGFR expression, organoids were split to single cells, left to grow five days on organoid medium, and then transferred to organoid medium with physiological EGF concentration $(0.63 \mathrm{ng} / \mathrm{mL})$. Five days later, organoids were collected and washed twice with $10 \mathrm{~mL}+/+/+$. RNA was extracted using RNeasy mini kit (Qiagen) according to protocol. RNA concentrations were measured using Nanodrop.

\section{cDNA Synthesis and Quantitative Polymerase Chain Reaction (PCR)}

For cDNA synthesis, RNA was incubated with $50 \mu \mathrm{g} / \mathrm{mL}$ Oligo(dT) 15 Primer (Promega) in water for $5 \mathrm{~min}$ at $70^{\circ} \mathrm{C}$. Subsequently GoScript Reverse Transcriptase (Promega) was used according to protocol to produce cDNA. qPCR reactions were performed in 384 well format using IQ SYBR green (Bio-Rad) in the presence of $0.67 \mu \mathrm{M} \mathrm{FW}$ and RV primer and cDNA transcribed from $25 \mathrm{ng}$ RNA. For qPCR, samples were incubated for $2 \mathrm{~min}$ at $95^{\circ} \mathrm{C}$ and for 40 cycles at: $15 \mathrm{~s}$ at $98{ }^{\circ} \mathrm{C}, 15 \mathrm{~s}$ at $58^{\circ} \mathrm{C}$ and $15 \mathrm{~s}$ at $72{ }^{\circ} \mathrm{C}$. Results were calculated by using the $\Delta \Delta \mathrm{Ct}$ method. Melt peak analysis was performed to assure that primers had no aspecific binding. The primers used are depicted in Table 1.

Table 1 Primer sequences used for quantitative Polymerase Chain Reaction.

\begin{tabular}{ll}
\hline Primer & Sequence '5 $\rightarrow$ '3 \\
\hline Human EGFR FW & AGGCAGGAGTAACAAGCTCAC \\
Human EGFR RV & ATGAGGACATAACCAGCCACC \\
Human GAPDH FW & GGAGCGAGATCCCTCCAAAAT \\
Human GAPDH RV & GGCTGTTGTCATACTTCTCATCG \\
\hline
\end{tabular}

EGFR: Epidermal Growth Factor Receptor, FW: Forward, RV: Reverse, GAPDH:

Glyceraldehyde 3-phosphate dehydrogenase.

\section{Photosensitizer Conjugation}

Monovalent NB 7D12, binding domain III on EGFR, and biparatopic NB 7D12-9G8, binding domains II and III on EGFR, were produced as previously described [16]. In short, the his-tagged nanobodies were produced in E. coli BL21 and purified from the periplasmic fraction using immobilized metal affinity chromatography (IMAC) with nickel-nitrilotriacetic acid agarose. $\mathrm{mAB}$ cetuximab, binding domain III on EGFR, was purchased at the local hospital pharmacy. As the original ligand of EGFR, EGF also binds domain III; 7D12, 7D12-9G8, and cetuximab all compete with EGF [16]. IRDye ${ }^{\circledR}$ 700DX NHS Ester (LI-COR) was conjugated to cetuximab, 7D12, and 7D12-9G8, as previously described [16], thus with molar ratio for conjugation of 1:4 for all conjugates. Alexa Fluor 647 NHS Ester (Thermo Fisher Scientific) was similarly conjugated to cetuximab. All conjugates were checked by gel electrophoresis after conjugation to determine the percentage of free dye, and to confirm the conjugates were not degraded, they were checked on gel every 3-4 weeks after conjugation. Conjugates were solely used when free dye percentage was less than $10 \%$. Additionally, to confirm that binding 
affinity was not affected after conjugation, new conjugates were tested in a binding assay on A431, as previously described [16].

\section{EGFR Flow Cytometry}

Organoids used for flow cytometry analysis were grown in physiological EGF medium containing $0.63 \mathrm{ng} / \mathrm{mL}$ EGF. Organoids were collected 7 days after splitting and disrupted into single cells using TrypLE. Cells were washed once with $10 \mathrm{~mL}+/+/+$, counted, and subsequently incubated in FACS buffer (PBS with 5\% FCS and 2 mM EDTA) containing $19 \mathrm{nM}$ cetuximab-Alexa647 for one hour on ice (100 $\mu$ L FACS buffer for 100,000 cells). After incubation, cells were washed once and resuspended in $100 \mu$ LACS buffer for analysis. Just before measurements, $1 \mu \mathrm{g} / \mathrm{mL}$ DAPI was added to allow the identification of dead cells that were excluded from the analysis. Unstained controls were taken along for each line and A431, 14C, and Hela served as positive controls. Measurements were carried out on the BD FACSCanto II (BioRad) with standard filter sets, and fluorescence intensity of cetuximab-Alexa647 was measured on the $633 \mathrm{~nm}$ channel. Analysis was performed using FlowJo software, BD Biosciences, Franklin Lakes, NJ, USA).

\section{Immunohistochemistry}

Organoids were cultured for one week on complete medium, followed by one week on physiological EGF medium. Subsequently, organoids were collected, washed twice to remove BME and incubated for at least $2 \mathrm{~h}$ at room temperature in 4\% PFA for fixation. Organoids were subsequently processed for paraffin embedding. Paraffin sections were stained with H\&E and the EGFR antibody (Invitrogen, clone 31G7, dilution 1:40, pretreatment pepsin). All stainings were performed at the pathology department of the University Medical Center Utrecht (UMCU).

\section{In Vitro PDT Assay on HNSCC Organoids}

Organoids were disrupted into single cells using TrypLE and manual shearing using a P1000 pipette. Single cells were subsequently plated in BME and cultured on physiological EGF medium without $\mathrm{N}$-acetyl-cysteine. Two days later, BME drops were mechanically disrupted by pipetting and $1 \mathrm{mg} / \mathrm{mL}$ dispase II (Sigma-Aldrich) was added to the medium to disrupt BME. Culture plates were incubated at $37^{\circ} \mathrm{C}$ for 40 min to digest the BME. Subsequently, organoids were collected, washed with cold $+/+/+$, and filtered using a $70 \mu \mathrm{m}$ nylon cell strainer. Organoids were counted and resuspended in 5\% $\mathrm{BME} /$ phyiological EGF medium without $\mathrm{N}$-acetyl-cysteine. Five hundred organoids were plated in a volume of $40 \mu \mathrm{L}$ in 384 well format using the multi-drop Combi Reagent Dispenser (Thermo Fisher Scientific). Average size at the start of treatment was approximately $50 \mu \mathrm{m}$. PS conjugates were added using HP Tecan D300e Digital Dispenser. Conjugates were dissolved in PBS containing 0.3\% Tween-20. Experiment was performed in technical triplicate. After two hours of incubation with the conjugates, $690 \mathrm{~nm}$ light was applied (Modulight), using a light fluence rate of $5 \mathrm{~mW} / \mathrm{cm} 2$ (measured by an Orion Laser power monitor). A total light dose of $26 \mathrm{~J} / \mathrm{cm} 2$ was given. Twenty-four hours after illumination, cell viability was measured using CellTiter-Glo 3D Reagent (Promega) according to protocol. Luminescence readout was carried out using a Spark multimode microplate reader (Tecan). Viability of wells that only received PBS/Tween for normalization was set at $100 \%$. Viability of organoids exposed to $10 \mu \mathrm{M}$ staurosporin was set at $0 \%$. A separate dark control plate was made, in which for each line the highest concentration of the conjugate was added in triplicate. GraphPad Prism (GraphPad Software Inc.) was used to create kill curves and lines were fitted by means of the nonlinear fit option ' $\log$ (inhibitor) vs. response -variable slope'. Concentration of the PS- 
conjugate was corrected for the DOC of the conjugate in order to plot on the $\mathrm{y}$-axis the total amount of PS, the actual drug.

\section{EGFR Overexpression Construct and Lentivirus Production}

cDNA was obtained from A431 cells. EGFR open reading frame was amplified using PCR (FW primer '5 $\rightarrow$ '3: GCTAGCGCCACCATGGACTACAAGGATGACGATGA CAAGATGCGACCCTCCGGGACGGC, Reverse primer '5 $\rightarrow$ '3: CACGCGTTGCT CCAATAAATTCACTGCTTT. PCR product was purified using gel extraction. Restriction digest using Nhe1 and Mlul allowed ligation of the EGFR open reading frame into the Addgene plasmid \#50661. Production of lentivirus was performed in HEK293T cells, which were transduced with packaging plasmids and the created EGFR construct. Transduction was performed using a mixture of $300 \mu \mathrm{L}$ PEI, $25 \mu \mathrm{g}$ EGFR construct, and $5 \mathrm{~mL}$ Optimem (Thermo-Fisher) that was added to the $15 \mathrm{~mL}$ of DMEM/10\% serum already placed on the HEK293T cells. After $8 \mathrm{~h}$, medium was refreshed. Three days later, supernatant was collected and filtered using a $0.40 \mu \mathrm{m}$ pore filter. Virus was collected by ultracentrifugation $\left(20.000 \times \mathrm{g}, 2 \mathrm{~h}, 4^{\circ} \mathrm{C}\right)$. Virus derived from one $15 \mathrm{~cm}$ dish of HEK293T cells was resuspended in $500 \mu \mathrm{L}$ organoid medium and stored at $-80{ }^{\circ} \mathrm{C}$ until use.

\section{Organoid Infection and Doxycycline-Mediated Induction of EGFR Expression}

The organoids were disrupted into single cells using TrypLE, washed, and incubated with $100 \mu \mathrm{L}$ virus suspension. Virus/cell mixture was incubated for $6 \mathrm{~h}$ in the presence of 1 $\mu \mathrm{g} / \mathrm{mL}$ polybyrene. After incubation, the organoids were washed with $10 \mathrm{~mL}+/+/+$ and plated in BME as usual. Three days later, the organoid medium supplemented with 1 $\mu \mathrm{g} / \mathrm{mL}$ puromycin (InvivoGen) was placed on the organoids. The organoids were kept on puromycin containing medium for two weeks, after which successful infection was validated using doxycycline induction. PDT was performed as previously described, except for the addition of $3 \mu \mathrm{g} / \mathrm{mL}$ doxycycline after splitting of the organoids two days prior to PDT. Doxycycline was also added during the PDT assay. For FACS analysis, organoids were cultured for one week in the presence of doxycycline.

\section{Results}

\section{EGFR Expression Differs between Patient-Derived Organoids from Different Donors and Recapitulates EGFR Levels of Respective Tissues}

Previously characterized HNSCC-derived organoids were cultured as described in [21]. Tumor-derived organoids were named $\mathrm{T}$ (tumor), while organoids obtained from normal tissues were named $\mathrm{N}$ (normal), followed by a number, which was identical to previously used in [21]. Tumor-status of the organoids was previously confirmed by DNA sequencing. This included targeted sequencing of EGFR for assessing amplification and mutations in exons 3, 7, 15, 18 to 21 (for more details on sequencing, see Supplementary Table S1). When available (i.e., in 3 out of 7 cases) organoids were established from tumor-adjacent wildtype epithelium. Wildtype status of these normal epithelium-derived organoids was confirmed by whole exome sequencing $(n=2)$ or Nutlin-3 selection $(n=$ 1). Nutlin-3 is an Mdm2-agonist preventing the growth of TP53 wildtype cells [26]. Hence, Nutlin-3 sensitive lines are TP53 wildtype. Relevant information on the organoids used in this study can be found in Table 2 . 
Table 2 Patient information corresponding to the organoid lines used in this study. From left to right, columns indicate: patient gender, patient age at diagnosis, tumor location, pretreatment, Human Pappiloma Virus (HPV) status of tumor, type of DNA sequencing used to confirm tumor status of the organoid line, and availability of organoids from only tumor $(\mathrm{T})$ or both normal epithelium and tumor (N/T).

\begin{tabular}{cccccccc}
\hline Organoid & Gender & Age & $\begin{array}{c}\text { Tumor } \\
\text { Location }\end{array}$ & $\begin{array}{c}\text { HPV } \\
\text { Status }\end{array}$ & $\begin{array}{c}\text { Sequencing } \\
\text { to Confirm } \\
\text { Tumor } \\
\text { Status }\end{array}$ & $\begin{array}{c}\text { Tumor } \\
\text { Status } \\
\text { Confirmed? }\end{array}$ & N/T \\
\hline 1 & male & 61 & tongue & negative & oncopanel & Yes & $\mathrm{T}$ \\
2 & female & 90 & larynx & negative & oncopanel & Yes & $\mathrm{T}$ \\
3 & female & 83 & larynx & negative & oncopanel & Yes & $\mathrm{T}$ \\
4 & male & 60 & tongue & negative & $\begin{array}{c}\text { oncopanel } \\
\text { exome }\end{array}$ & Yes & N/T \\
5 & male & 80 & $\begin{array}{c}\text { parotid } \\
\text { gland }\end{array}$ & negative & $\begin{array}{c}\text { Yes } \\
\text { sequencing }\end{array}$ & N/T \\
& male & 82 & $\begin{array}{c}\text { oral } \\
\text { cavity }\end{array}$ & negative & oncopanel & Yes & $\mathrm{T}$ \\
8 & female & 70 & gingiva & negative & $\begin{array}{c}\text { exome } \\
\text { sequencing }\end{array}$ & Yes & N/T \\
\hline
\end{tabular}

To assess EGFR expression levels in HNSCC organoids, both quantitative PCR and flow cytometry were performed. EGFR messenger RNA was detectable in all tested organoid lines, although expression varied between organoids derived from different donors (Figure S1a). Interestingly, lowering the level of EGF in organoid culture medium to the levels detected in human serum (hereafter called 'physiological EGF'), resulted in upregulation of EGFR expression in all, but two lines (Figure S1b). This finding was in line with the fact that EGFR protein on organoids could initially not be detected by flow cytometry, whereas it was detectable on control cell lines overexpressing EGFR. Indeed, EGFR protein levels were increased upon culture of organoids in physiological EGF medium (Figure 1a). In line with variable EGFR expression in primary tumors [27], EGFR protein levels varied between organoids derived from different donors (Figure 1b). EGFR protein levels detected on HNSCC-derived organoids were lower than those observed on cell lines expressing high (A431) and moderate (14C) EGFR levels (indicated by '2D cell lines' in Figure 1b). Expression is shown relative to HeLa cells, which have been reported to have physiological levels of EGFR expression. All these cell lines have previously been used to assess efficacy and selectivity of EGFR-targeted PDT in vitro $[16,17]$.

As organoid culture allows for the expansion of corresponding normal tissue in culture, EGFR levels of organoid grown from surrounding normal tissues were also measured. For three donors, EGFR levels on both normal and tumor organoids were compared. In all cases, EGFR protein levels detected on tumor cells were higher than those detected on matched normal organoids (Figure 1b). Importantly, EGFR expression levels in organoids (colored bars) were comparable to EGFR levels of primary patient material samples (black bars indicated with 'primary tissue', Figure 1b). The observed differences in EGFR expression between normal and tumor organoids were confirmed using immunohistochemistry (Figure 1c). 


\section{Organoid Response to EGFR-Targeted PDT Is Donor-Dependent and Tumor- Specific}

Existing PDT protocols of 2D cell lines were adjusted to make them suitable for organoid screens (Figure 2a). All experiments were performed with cetuximab-PS (which is currently tested in the clinic), and the nanobody-PS conjugates 7D12-PS and 7D12-9G8PS, that were used in our previous studies [16]. 7D12 is a monovalent EGF-competing nanobody, whereas 7D12-9G8-PS is a biparatopic EGF-competing nanobody, consisting of two genetically fused monomeric nanobodies targeting two different epitopes on EGFR. In vitro, biparatopic nanobodies have been shown to be more potent than monovalent nanobodies [16], as they can carry more PS per nanobody and can promote receptor clustering, which leads to faster EGFR endocytosis and thus, conjugate internalization [28].

Upon exposure to EGFR-targeted PDT, organoids were killed by concentrations of PS-conjugate that did not result in cell death in the dark (Figure 2b, shown for 7D12-9G8PS, but found for all used conjugates). As expression levels are lower in patient-derived organoids than in the cell lines where efficacy of these nanobody-PS conjugates has been observed before [16], these findings are encouraging and of clinical relevance.

Tumor and wildtype organoids established from the same patient were subjected in parallel to EGFR-targeted PDT. In all pairs tested, tumor organoids were found to be more sensitive to PDT than their wildtype counterparts (Figure 2c). Using area under the curve (AUC) as a quantitative measure of in vitro killing, tumor organoids clustered separately from wildtype organoids, for all PS-conjugates tested (Figure 2d). Although numbers are limited, these findings are encouraging and provide the first indication that cancer cells derived from patient material can be selectively killed by targeted PDT, leaving normal cells unaffected. For all organoids tested, the effect of nanobody-targeted PDT was more pronounced than that of antibody-targeted PDT. A comparison between the two nanobody-PS conjugates points to the treatment with the biparatopic nanobody 7D12-9G8-PS, as this is the most effective in most of the organoids tested. 
(a)
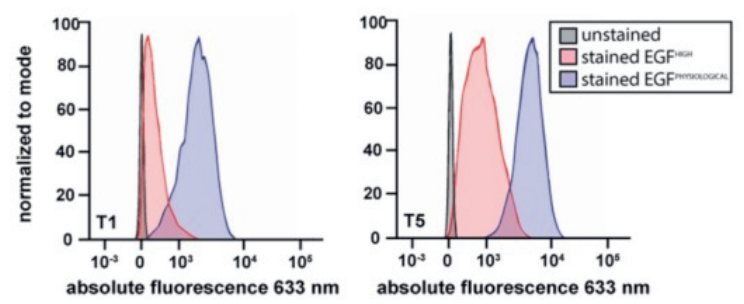

(b)

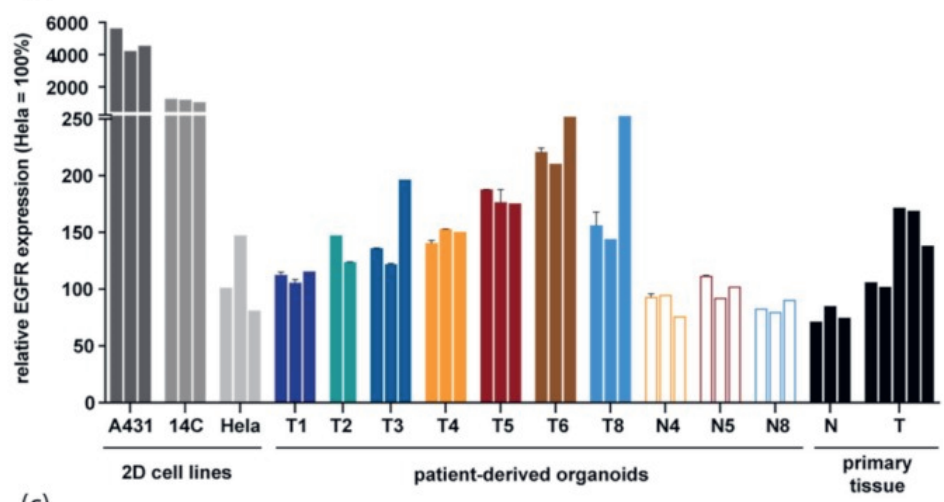

(c)

tissue

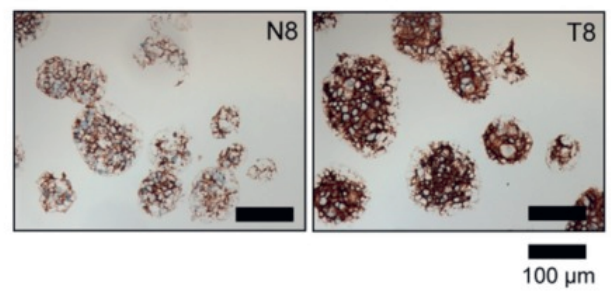

Figure 1 Epidermal Growth Factor Receptor (EGFR) expression differs between patient-derived organoids from different donors and recapitulates EGFR levels of respective tissues. (a) EGFR protein expression detected by flow cytometry. EGFR expression of organoids grown in either physiological Epidermal Growth Factor (EGF) (blue peak) or high EGF (ref peak) medium is shown for two HNSCC organoid lines. An unstained control is shown in black. (b) EGFR protein expression measured by flow cytometry in 2D cell lines commonly used in in vitro EGFR-targeted PDT studies, organoid lines derived from HNSCC patients both normal and tumor, and in primary tissue samples. For organoids, the experiment was performed in technical duplicate (error bars) and biological triplicate (individual bars). EGFR expression was stable over time, as biological replicates were measured two months apart. EGFR protein levels are shown relative to HeLa cells (set at 100\%). Results of tumor organoids are shown in filled bars, results of wildtype organoids are shown in clear, outlined bars. For primary tissues, each bar represents EGFR expression on a tissue sample derived from an individual patient. (c) EGFR immunohistochemical staining performed on N8 and T8 organoids. Scalebar, $100 \mu \mathrm{m}$. 
(a)

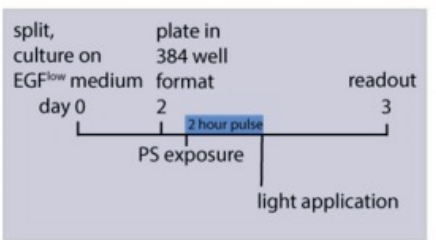

(c)
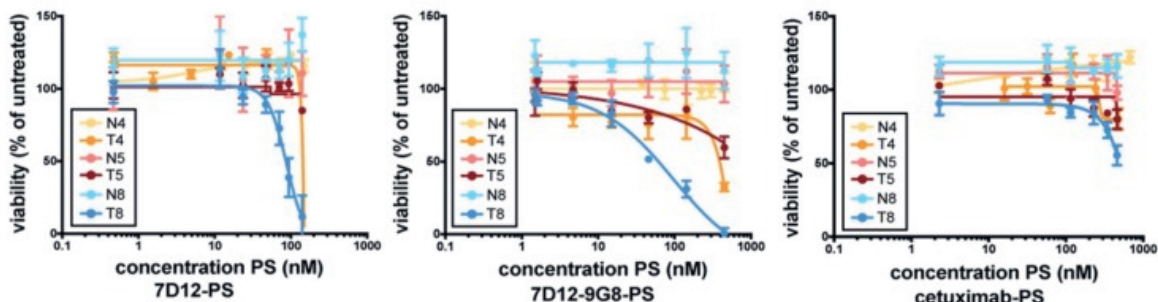

(d)

\begin{tabular}{|c|c|c|c|c|c|c|}
\hline \multirow{2}{*}{ organoid } & \multicolumn{6}{|c|}{ 7D12-PS } \\
\hline & $T 8$ & T4 & T5 & N5 & N8 & N4 \\
\hline \multirow[t]{2}{*}{ AUC } & 8966 & 11490 & 14182 & 15764 & 16718 & \begin{tabular}{|l|}
17657 \\
\end{tabular} \\
\hline & \multicolumn{6}{|c|}{ 7D12-9G8-PS } \\
\hline organoid & T8 & T4 & T5 & N4 & N5 & N8 \\
\hline \multirow[t]{2}{*}{ AUC } & 12167 & 28523 & 34247 & 43985 & 48186 & 53548 \\
\hline & \multicolumn{6}{|c|}{ Cetuximab-PS } \\
\hline organoid & T8 & T5 & T4 & N5 & N8 & N4 \\
\hline AUC & 36296 & 41345 & 45305 & 50064 & 53091 & 66487 \\
\hline
\end{tabular}

Figure 2 Organoid response to in vitro EGFR-targeted PDT is donor-dependent and tumorspecific. (a) Schematic outline of the experimental set-up of organoid in vitro PDT. Organoids are disrupted into single cells, recovered for two days on medium containing physiological EGF, and subsequently filtered, counted, and plated into a 384 well format. A two-hour exposure to EGFR-targeting nanobody-PS or antibody-PS conjugates was followed by a light-dose activating the PS. Twenty-four hours later, cell viability was assessed. (b) EGFR-targeting conjugates used in this study did not show toxicity without activation of the PS. Here, toxicity of 7D12-9G8 in T3 and T4 is shown as an example. (c) Response to EGFR-targeted PDT of matched normal and tumor organoid pairs. Response to 7D12-PS, 7D12-9G8-PS, and cetuximab-PS is shown for N4 and T4 (orange), N5 and T5 (red), and N8 and T8 (blue). Normal organoids are depicted in a lighter shade of the same color than their tumor counterparts. (d) Quantification of organoid response to EGFR-targeted PDT shown in Figure 2c Area under the curve (AUC) is used as a readout for response to EGFR-targeted PDT. Blue indicates low AUC-values (response to therapy), whereas red indicates high AUC values (no response to therapy).

\section{Organoid Response to EGFR-Targeted PDT Correlates with EGFR Expression Levels}

The number of patient-derived tumor organoids was further expanded and their response to targeted PDT assessed (Figure 3). Organoids derived from different patients showed variable responses to EGFR-targeted PDT. Comparison of EGFR protein levels and 
response to therapy revealed a positive correlation between these two variables, confirming results previously obtained in 2D cell lines [16]. Organoids that express higher protein levels of EGFR were more sensitive to EGFR-targeted PDT, regardless of the PS-conjugate used (Figure 3a,b). Since organoids express EGFR at comparable levels to primary patient tissue, these results are clinically relevant, as they suggest that EGFR levels could be a predictor for EGFR-targeting PDT. The response of all individual organoids (both tumor and normal) to the three used PS-conjugates is shown in Figure S2.

(a)

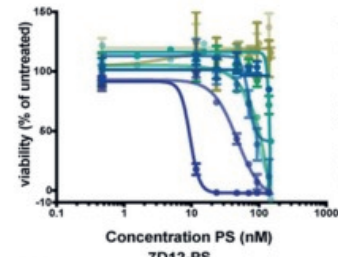

(b)

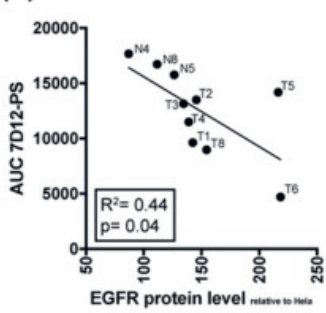

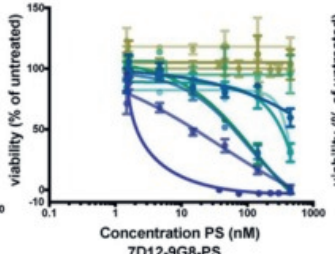

7D12-9G8-PS

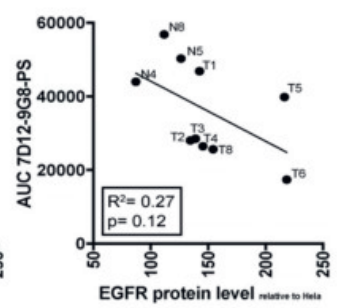

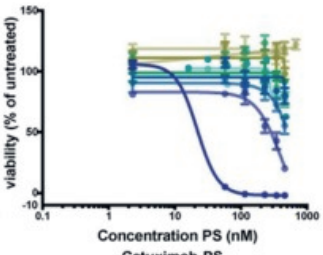

Cetuximab-PS
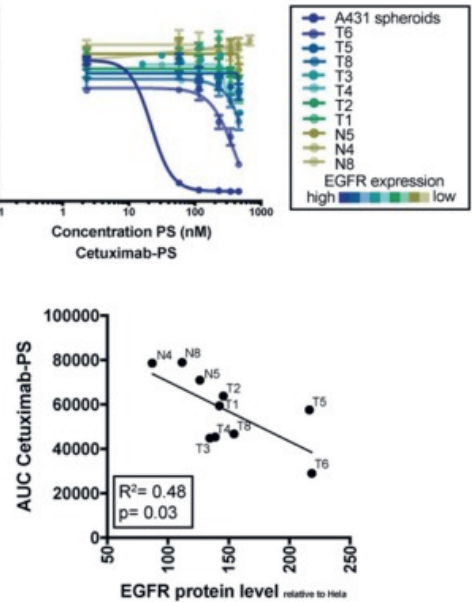

Figure 3 Organoid response to in vitro EGFR-targeted PDT correlates with EGFR expression levels. (a) Sensitivity of HNSCC organoids with variable EGFR expression to PDT using conjugates 7D12-PS (first panel), 7D12-9G8-PS (second panel), and cetuximab-PS (third panel). Color of the lines indicate the relative EGFR expression level, detected by FACS analysis (blue $=$ highest expression, yellow $=$ lowest expression). (b) Correlation plots showing the relation between EGFR expression on the x-axis and response to PDT therapy, as indicated by area under the curve (AUC) on the y-axis. The first panel shows the AUC for 7D12-PS, second panel for 7D12-9G8-PS, and the third panel for cetuximab-PS.

\section{Induction of EGFR Expression Increases Sensitivity to EGFR-Targeted PDT}

Based on the observed correlation between EGFR expression and the targeted PDT response, we set out to verify whether an increase in EGFR expression would result in increased sensitivity to EGFR-targeted PDT. For this, a doxycycline-inducible EGFR expression construct was introduced into two HNSCC organoids, T6 and T8 (Figure 4a). Subsequent doxycycline-induction resulted in GFP expression (A GFP-coding sequence was cloned after EGFR in the used construct), indicating that the induction was effective (Figure 4b). After doxycycline treatment, EGFR protein levels increased in both lines (Figure 4c). Lastly, EGFR overexpression resulted in increased sensitivity to targeted PDT (Figure 4d). This data confirms that EGFR-targeted PDT is most effective in organoids having high expression of EGFR, showing a causal relation between EGFR levels and sensitivity to EGFR-targeted PDT. 
(a)

inducible EGFR-GFP expression

T2A

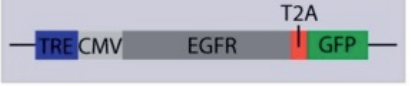

(c)

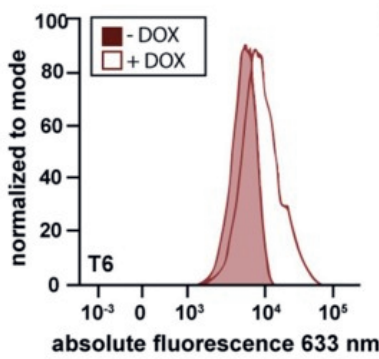

(d)

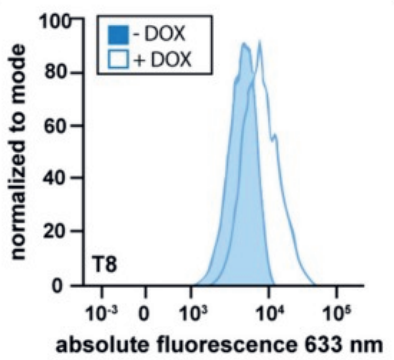

(b)
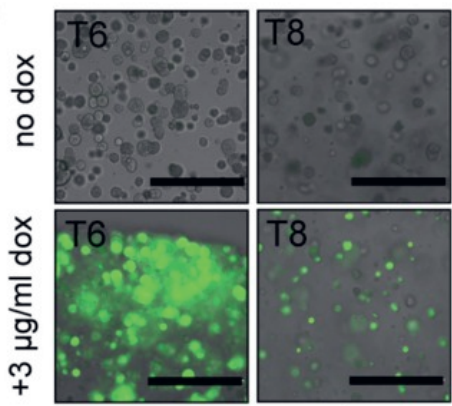

$\overline{400 \mu \mathrm{m}}$

(e)

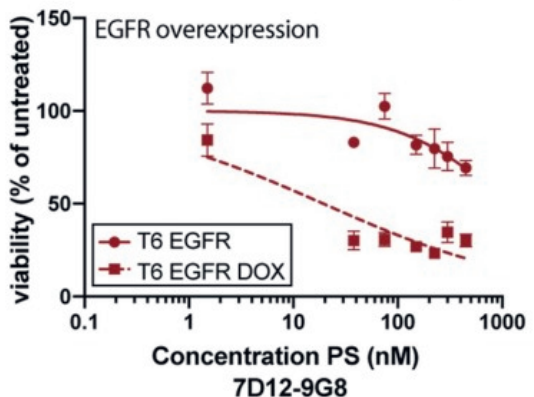

(f)

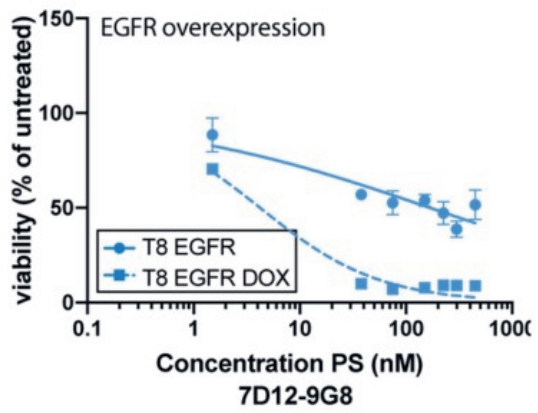

Figure 4 Induced EGFR expression enhances the response to EGFR-targeted PDT. (a) Schematic outline of lentiviral infector used to create organoid lines that can be induced to overexpress EGFR (TRE: tetracycline responsive element, CMV: cytomegalovirus promotor). The TRE and CMV promotor precede the open reading frame encoding EGFR and GFP, separated by a T2A sequencing, assuring generation of separate mRNA molecules for EGFR and GFP. (b) Merged brightfield/immunofluorescence images of organoids after two-day administration of doxycycline. Scalebar $400 \mu \mathrm{m}$. (c) and (d) Effect of doxycycline-mediated EGFR overexpression on EGFR protein levels in organoid lines T6 and T8, respectively. Colored peaks indicate uninduced expression, lined peaks indicate induction of EGFR expression. (e) and (f) EGFRtargeted PDT using nanobody 7D12-9G8-PS in EGFR overexpressing organoid lines T6 and T8. Organoids were either doxycycline-induced (squared symbols, dashed line) or uninduced (round symbols, solid line) and exposed to PDT as previously described. 


\section{Discussion}

The first aim of this study was to characterize EGFR expression in HNSCC patientderived organoids and compare the expression level to that of cell lines used in our previous studies. EGFR levels in organoids were found to recapitulate EGFR expression levels of both tumor and normal patient material samples, even when retained in culture. Notably, EGFR expression levels were found to be lower in patient-derived organoids than in cell lines where efficacy of these nanobody-PS conjugates has been observed before [16]. The EGFR levels detected on organoids were comparable to expression levels detected on HeLa cells, a cell line that has been found to be unaffected by nanobodytargeted PDT under specific conditions [15]. Subsequently, we aimed to investigate EGFR-targeted PDT in this novel 3D model; in the lower levels of EGFR expression, EGFR-targeted PDT was shown to be effective in these patient-derived organoids. Tumor organoids were more sensitive to PDT than their corresponding wildtype counterparts, suggesting that, with appropriate dosing, this therapy is expected to leave the normal epithelium surrounding the tumor unaffected. EGFR levels between organoids from different patients varied, and could be correlated with response to EGFR-targeted PDT. Higher levels of EGFR protein correlated with a better response to this therapy in vitro. Finally, we aimed to verify the correlation between EGFR expression levels and PDT efficacy. For that, inducible EGFR overexpression was employed, which resulted in increased PDT efficacy.

The known clinical need for effective and selective local treatment of HNSCC, and the complexity of conventional PDT protocols used in the clinic encourages the development of more targeted PDT approaches. This study complements others that have shown selective and extensive tumor damage as a result of EGFR-targeted PDT [29-34]. However, here we have taken the unique approach of testing PDT in (matching) patientderived tumor and normal cells. To our knowledge, this is the first report of in vitro PDT in a 3D patient-derived model, although several studies have investigated PDT or targeted PDT in spheroid models grown from 2D cell lines [22-25]. When compared to the 2D cultures of which they were derived, these $3 \mathrm{D}$ spheroids certainly better recapitulate a mass of tumor cells in vivo. However, this study highlights the possible differences in target expression levels between such 3D spheroids and patient-derived organoids, where the expression detected in the latter was found to better recapitulate expression levels detected on primary tissue. Thus, the use of patient-derived models should be encouraged in the development and evaluation of targeted therapies.

Gene amplification of EGFR is reported in $15 \%$ of HNSCC patients [4], although EGFR protein overexpression has been reported in up to $90 \%$ of tumors [5]. Here, tumor cells showed increased EGFR expression levels compared to their wildtype counterparts, despite the fact that none of the tested organoids showed any evidence for EGFR amplification [21]. These findings are in line with previously mentioned statistics and support the hypothesis that EGFR protein overexpression is not only driven by gene amplification. It would be of interest to test EGFR-targeted PDT in organoids derived from a tumor carrying EGFR amplification, to see if responses are comparable to those observed in the organoids reported here.

In this work, we confirmed the correlation that was previously observed between EGFR expression and response to EGFR-targeting PDT [16,17]. Here, we confirm this correlation in patient-derived organoids. These findings suggest that high EGFR expression in tumor tissue might be used as a predictive marker for response to EGFRtargeted PDT. The potential of EGFR expression as a biomarker for other EGFR-targeted therapies was previously investigated [5]. Such studies found that EGFR expression levels could not predict response to the EGFR-blocking antibody cetuximab. This is likely 
explained by commonly found downstream mutations in the EGFR pathway, which will negate the effect of cetuximab competing with EGF for EGFR binding, even in EGFR overexpressing tumors [4]. Since the effect of EGFR-targeted PDT is independent from EGFR downstream signaling and mutations, EGFR expression could potentially be a suitable biomarker for response to EGFR-targeted PDT. EGFR-targeted PDT is expected to be an effective treatment option, even for tumors carrying EGFR-downstream mutations.

Cetuximab-targeted PDT is currently tested in clinical trials (NCT02422979). Initial findings are encouraging and show responses to therapy, with limited side-effects [15]. Here, no inclusion criteria were applied based on EGFR expression levels. It would be of value to see to what extent EGFR levels can explain differential responses to therapy in this patient cohort. To determine if organoids can aid personalized decision-making, it would be interesting to establish organoids from patients receiving EGFR-targeted PDT. As such, it would be possible to compare in vitro organoid responses to corresponding patient responses and see if organoids hold predictive potential in EGFR-targeted PDT.

Even though PDT is a local therapy, evidences have pointed to the potential of it triggering an immune response that can lead to systemic effects and even a memory to protect patients from tumor recurrences [35]. For patients already diagnosed with metastatic disease, a combination of the standard systemic chemotherapy with PDT could be foreseen. Such combinations of chemotherapy and PDT have already been explored in several preclinical and clinical studies (as reviewed in [36]). Overall, the addition of PDT has resulted in increased response rates when compared to chemotherapy alone, even in advanced disease where metastasis are present [36]. Combination treatment often results in more severe side-effects than single agent therapy, thereby hampering clinical applicability. As the use of targeted PDT has shown less side-effects than conventional PDT [15], it is suggested that the introduction of targeted PDT in combination with chemotherapy will be better tolerated than conventional PDT combined with chemotherapy.

The efficiency of organoid culture allows for the establishment of matching organoid pairs, where corresponding normal oral mucosa tissue can be grown in parallel to the adjacent tumor tissue. Here, we tested three matching organoid pairs, and found in all cases that wildtype organoids showed no response to EGFR-targeted PDT. This is likely due to the lower EGFR expression levels detected on wildtype cells, although others have shown that tumor cells also produce more ROS than wildtype cells, which might also influence PDT responses [37,38].

Patient-derived organoids create the opportunity to compare the response of both tumor and matching wildtype cells. These allow to study the specificity of EGFR-targeted PDT better than, for example, transplantation of patient-derived tumor cells in mice, where the species difference can contribute to differential response of (human) tumor and (mouse) wildtype cells. On the contrary, in vivo studies are better suited than in vitro models to evaluate distribution and the effect of bio-availability of PS-conjugates. A possible solution could be transplantation of both the normal and tumor organoid pairs in order to investigate these aspects in vivo [21].

In this study, we used two different nanobodies: monovalent 7D12 and biparatopic 7D12-9G8. In vitro, biparatopic nanobodies have been shown to be more potent than monovalent nanobodies [16], as they can carry more PS per nanobody, and their binding to EGFR results in receptor clustering, leading to faster EGFR (and conjugate) endocytosis [28]. The internalization of nanobody-PS and also of antibody-PS has been correlated with increased cellular damage [39,40]. However, in vivo, only small differences have been observed thus far between these two nanobody formats, where 
7D12-PS has shown more reproducible tumor damage than 7D12-9G8-PS. As 7D12 is smaller than 7D12-9G8, tissue penetration of this nanobody is suggested to be more rapid and more homogenous [41]. Compared to treatment with antibody-PS, nanobody-PS results in less variation in the extent of damage induced and in an increase in tumor damage [17]. These results have been correlated to the larger size of the antibody, hampering a homogenous distribution in vivo. Also, in organoids, cetuximab-PS was found to be less effective than any of the two nanobodies (Figure S2). In contrast to previously described in vivo studies, we found that, in vitro, the biparatopic nanobody more effectively killed HNSCC cells than the monovalent nanobody. This is likely due to the small dimensions of the organoids employed, and the fact that this set up does not reflect systemic distribution. Nevertheless, besides our study focused on targeted PDT, organoids are gaining increasingly more attention as realistic representations of tumors that enable effective drug screens, with a variety of drugs [21,42-52].

While the predictive potential of patient-derived organoids has been shown earlier for cystic fibrosis $[53,54]$, recent data shows that organoids also hold predictive potential in oncological therapeutic context $[21,44-46,48]$. In these studies, a correlation between patient response and the response of matching organoids that were exposed to the same therapy was observed. Although numbers are limited, future studies will show if such a correlation holds true in a larger cohort of patients and for more tumor types.

\section{Conclusions}

Here we present HNSCC-derived organoids as a clinically relevant 3D model for in vitro targeted PDT. We show that EGFR expression levels detected on patient-derived HNSCC organoids closely recapitulate EGFR expression detected in primary tissue. EGFR expression levels were found to correlate to the response to EGFR-targeted PDT. Wildtype organoids grown from tumor-adjacent normal tissues showed lower EGFR expression levels than their tumor counterparts, and were not affected by PDT. Taken together, patient-derived organoids are shown to be a useful model for EGFR-targeted PDT research.

\section{Acknowledgments}

We thank Folkert Morsink and Emma de Ruiter of the Department of Pathology at the UMCU for his help with performing immunohistochemical stainings. We would like to thank Sigrid Kolders for her help with drug screens. We thank Anne Snelting and Anneta Brousali for their help with patient inclusion. We thank Stefan van der Elst for his help with FACS analysis.

\section{References}

1. Squier, C.; Kremer, M. Biology of oral mucosa and esophagus. J. Natl. Cancer Inst. Monogr. 2001, 29, 7-15.

2. Tijink, B.M.; Buter, J.; de Bree, R.; Giaccone, G.; Lang, M.S.; Staab, A.; Leemans, C.R.; van Dongen, G.A.M.S. A phase I dose escalation study with anti-CD44v6 bivatuzumab mertansine in patients with incurable squamous cell carcinoma of the head and neck or esophagus. Clin. Cancer Res. 2006, 12, 6064-6072.

3. Guan, J.; Li, Q.; Zhang, Y.; Xiao, N.; Chen, M.; Zhang, Y.; Li, L.; Chen, L. A meta-analysis 
comparing cisplatin-based to carboplatin-based chemotherapy in moderate to advanced squamous cell carcinoma of head and neck (SCCHN). Oncotarget 2016, 7, 7110-7119.

4. The Cancer Genome Atlas Network Comprehensive genomic characterization of head and neck squamous cell carcinomas. Nature 2015, 517, 576-582.

5. Bossi, P.; Resteghini, C.; Paielli, N.; Licitra, L.; Pilotti, S.; Perrone, F. Prognostic and predictive value of EGFR in head and neck squamous cell carcinoma. Oncotarget 2016, 7, 74362-74379.

6. Mandic, R.; Rodgarkia-Dara, C.J.; Zhu, L.; Folz, B.J.; Bette, M.; Weihe, E.; Neubauer, A.; Werner, J.A. Treatment of HNSCC cell lines with the EGFR-specific inhibitor cetuximab (Erbitux $\left.{ }^{\circledR}\right)$ results in paradox phosphorylation of tyrosine 1173 in the receptor. FEBS Lett. 2006, 580, 4793-4800.

7. Bonner, J. a; Harari, P.M.; Giralt, J.; Azarnia, N.; Shin, D.M.; Cohen, R.B.; Jones, C.U.; Sur, R.; Raben, D.; Jassem, J.; et al. Radiotherapy plus cetuximab for squamous-cell carcinoma of the head and neck. N. Engl. J. Med. 2006, 354, 567-578.

8. Argiris, A.; Karamouzis, M. V.; Raben, D.; Ferris, R.L. Head and neck cancer. Lancet (London, England) 2008, 371, 1695-709.

9. $\quad$ Plaetzer, K.; Krammer, B.; Berlanda, J.; Berr, F.; Kiesslich, T. Photophysics and photochemistry of photodynamic therapy: fundamental aspects. Lasers Med. Sci. 2009, 24, 259-268.

10. Trachootham, D.; Alexandre, J.; Huang, P. Targeting cancer cells by ROS-mediated mechanisms: A radical therapeutic approach? Nat. Rev. Drug Discov. 2009, 8, 579-591.

11. Curtin, J.F.; Donovan, M.; Cotter, T.G. Regulation and measurement of oxidative stress in apoptosis. J. Immunol. Methods 2002, 265, 49-72.

12. Agostinis, P.; Berg, K.; Cengel, K.A.; Foster, T.H.; Girotti, A.W.; Gollnick, S.O.; Hahn, S.M.; Hamblin, M.R.; Juzeniene, A.; Kessel, D. Photodynamic therapy of cancer: an update. CA. Cancer J. Clin. 2011, 61, 250-281.

13. Mew, D.; Wat, C.-K.; Towers, G.H.; Levy, J.G. Photoimmunotherapy: treatment of animal tumors with tumor-specific monoclonal antibody-hematoporphyrin conjugates. J. Immunol. 1983, 130, $1473-1477$.

14. van Dongen, G.A.M.S.; Visser, G.W.M.; Vrouenraets, M.B. Photosensitizer-antibody conjugates for detection and therapy of cancer. Adv. Drug Deliv. Rev. 2004, 56, 31-52.

15. Cognetti, D.; Curry, J.M.; Gillenwater, A.M.; William, W.N.; Kochuparambil, S.T.; McDonald, D.; Fidler, M.; Stenson, K.M.; Vasan, N.R.; Razaq, M.A.; et al. A phase 2a, multicenter, open-label study of RM-1929 photoimmunotherapy in patients with recurrent head and neck cancer. Int. J. Radiat. Oncol. 2018, 100, 1368.

16. Heukers, R.; van Bergen en Henegouwen, P.M.P.; Oliveira, S. Nanobody-photosensitizer conjugates for targeted photodynamic therapy. Nanomedicine Nanotechnology, Biol. Med. 2014, 10, 1441-1451.

17. Van Driel, P.B.A.A.; Boonstra, M.C.; Slooter, M.D.; Heukers, R.; Stammes, M.A.; Snoeks, T.J.A.; De Bruijn, H.S.; Van Diest, P.J.; Vahrmeijer, A.L.; en Henegouwen, P.M.P. van B. EGFR targeted nanobody-photosensitizer conjugates for photodynamic therapy in a pre-clinical model of head and neck cancer. J. Control. Release 2016, 229, 93-105.

18. Hamers-Casterman, C.; Atarhouch, T.; Muyldermans, S.; Robinson, G.; Hammers, C.; Songa, E.B.; Bendahman, N.; Hammers, R. Naturally occurring antibodies devoid of light chains. Nature 1993, $363,446-448$.

19. Carpenter, G.; Cohen, S. Epidermal growth factor. Annu. Rev. Biochem. 1979, 48, 193-216.

20. Kretzschmar, K.; Clevers, H. Organoids: Modeling Development and the Stem Cell Niche in a Dish. Dev. Cell 2016, 38, 590-600.

21. Driehuis, E.; Kolders, S.; Spelier, S.; Lõhmussaar, K.; Willems, S.M.; Devriese, L.A.; de Bree, R.; de Ruiter, E.J.; Korving, J.; Begthel, H.; et al. Oral mucosal organoids as a potential platform for personalized cancer therapy. Cancer Discov. 2019, 9, 852-871.

22. Broekgaarden, M.; Rizvi, I.; Bulin, A.L.; Petrovic, L.; Goldschmidt, R.; Massodi, I.; Celli, J.P.; Hasan, T. Neoadjuvant photodynamic therapy augments immediate and prolonged oxaliplatin efficacy in metastatic pancreatic cancer organoids. Oncotarget 2018, 9, 13009-13022.

23. Zuchowska, A.; Jastrzebska, E.; Chudy, M.; Dybko, A.; Brzozka, Z. 3D lung spheroid cultures for evaluation of photodynamic therapy (PDT) procedures in microfluidic lab-on-a-chip system. Anal. 
Chim. Acta 2017, 990, 110-120.

24. Sato, K.; Hanaoka, H.; Watanabe, R.; Nakajima, T.; Choyke, P.L.; Kobayashi, H. Near infrared photoimmunotherapy in the treatment of disseminated peritoneal ovarian cancer. Mol. Cancer Ther. 2015, 14, 141-150.

25. Millard, M.; Yakavets, I.; Piffoux, M.; Brun, A.; Gazeau, F.; Guigner, J.-M.; Jasniewski, J.; Lassalle, H.-P.; Wilhelm, C.; Bezdetnaya, L. mTHPC-loaded extracellular vesicles outperform liposomal and free mTHPC formulations by an increased stability, drug delivery efficiency and cytotoxic effect in tridimensional model of tumors. Drug Deliv. 2018, 25, 1790-1801.

26. Vassilev, L.T.; Vu, B.T.; Graves, B.; Carvajal, D.; Podlaski, F.; Filipovic, Z.; Kong, N.; Kammlott, U.; Lukacs, C.; Klein, C.; et al. In vivo activation of the $\mathrm{p} 53$ pathway by small-molecule antagonists of MDM2. Science (80-. ). 2004, 303, 844-848.

27. Kalyankrishna, S.; Grandis, J.R. Epidermal growth factor receptor biology in head and neck cancer. J Clin Oncol 2006, 24, 2666-2672.

28. Heukers, R.; Vermeulen, J.F.; Fereidouni, F.; Bader, A.N.; Voortman, J.; Roovers, R.C.; Gerritsen, H.C.; Van Bergen En Henegouwen, P.M.P. Endocytosis of EGFR requires its kinase activity and N-terminal transmembrane dimerization motif. J. Cell Sci. 2013, 126, 4900-4912.

29. Gijsens, A.; Missiaen, L.; Merlevede, W.; De Witte, P. Epidermal growth factor-mediated targeting of chlorin e6 selectively potentiates its photodynamic activity. Cancer Res. 2000, 60, 2197-2202.

30. Hemming, A.W.; Davis, N.L.; Dubois, B.; Quenville, N.F.; Finley, R.J. Photodynamic therapy of squamous cell carcinoma. An evaluation of a new photosensitizing agent, benzoporphyrin derivative and new photoimmunoconjugate. Surg. Oncol. 1993, 2, 187-196.

31. Kameyama, N.; Matsuda, S.; Itano, O.; Ito, A.; Konno, T.; Arai, T.; Ishihara, K.; Ueda, M.; Kitagawa, Y. Photodynamic therapy using an anti-EGF receptor antibody complexed with verteporfin nanoparticles: a proof of concept study. Cancer Biother. Radiopharm. 2011, 26, 697704.

32. Master, A.; Malamas, A.; Solanki, R.; Clausen, D.M.; Eiseman, J.L.; Sen Gupta, A. A cell-targeted photodynamic nanomedicine strategy for head and neck cancers. Mol. Pharm. 2013, 10, 19881997.

33. Springa, B.Q.; Abu-Yousif, A.O.; Palanisami, A.; Rizvi, I.; Zheng, X.; Mai, Z.; Anbil, S.; Sears, R.B.; Mensah, L.B.; Goldschmidt, R.; et al. Selective treatment and monitoring of disseminated cancer micrometastases in vivo using dual-function, activatable immunoconjugates. Proc. Natl. Acad. Sci. U. S. A. 2014, 111, 933-942.

34. Mitsunaga, M.; Ogawa, M.; Kosaka, N.; Rosenblum, L.T.; Choyke, P.L.; Kobayashi, H. Cancer cell-selective in vivo near infrared photoimmunotherapy targeting specific membrane molecules. Nat. Med. 2011, 17, 1685-1691.

35. Maeding, N.; Verwanger, T.; Krammer, B. Boosting Tumor-Specific Immunity Using PDT. Cancers (Basel). 2016, 8, 91.

36. Luo, D.; Carter, K.A.; Miranda, D.; Lovell, J.F. Chemophototherapy: an emerging treatment option for solid tumors. Adv. Sci. 2017, 4, 1600106.

37. Schumacker, P.T. Reactive oxygen species in cancer cells: live by the sword, die by the sword. Cancer Cell 2006, 10, 175-176.

38. Szatrowski, T.P.; Nathan, C.F. Production of large amounts of hydrogen peroxide by human tumor cells. Cancer Res. 1991, 51, 794-798.

39. Carcenac, M.; Dorvillius, M.; Garambois, V.; Glaussel, F.; Larroque, C.; Langlois, R.; Hynes, N.E.; Van Lier, J.E.; Pèlegrin, A. Internalisation enhances photo-induced cytotoxicity of monoclonal antibody-phthalocyanine conjugates. Br. J. Cancer 2001, 85, 1787-1793.

40. Vrouenraets, M.B.; Visser, G.W.M.; Stigter, M.; Oppelaar, H.; Snow, G.B.; Van Dongen, G.A.M.S. Targeting of aluminum (III) phthalocyanine tetrasulfonate by use of internalizing monoclonal antibodies: Improved efficacy in photodynamic therapy. Cancer Res. 2001, 61, 1970-1975.

41. Beltrán Hernández, I.; Rompen, R.; Rossin, R.; Xenaki, K.T.; Katrukha, E.A.; Nicolay, K.; van Bergen en Henegouwen, P.; Grüll, H.; Oliveira, S.; Beltran Hernandez, I. Imaging of tumor spheroids, dual-isotope SPECT, and autoradiographic analysis to assess the tumor uptake and distribution of different nanobodies. Mol. Imaging Biol. 2019.

42. Van De Wetering, M.; Francies, H.E.; Francis, J.M.; Bounova, G.; Iorio, F.; Pronk, A.; Van Houdt, 
W.; Van Gorp, J.; Taylor-Weiner, A.; Kester, L.; et al. Prospective derivation of a living organoid biobank of colorectal cancer patients. Cell 2015, 161, 933-945.

43. Mullenders, J.; de Jongh, E.; Brousali, A.; Roosen, M.; Blom, J.P.A.; Begthel, H.; Korving, J.; Jonges, T.; Kranenburg, O.; Meijer, R.; et al. Mouse and human urothelial cancer organoids: A tool for bladder cancer research. Proc. Natl. Acad. Sci. 2019, 116, 4567-4574.

44. Ooft, S.N.; Weeber, F.; Dijkstra, K.K.; McLean, C.M.; Kaing, S.; van Werkhoven, E.; Schipper, L.; Hoes, L.; Vis, D.J.; van de Haar, J.; et al. Patient-derived organoids can predict response to chemotherapy in metastatic colorectal cancer patients. Sci. Transl. Med. 2019, 11, eaay2574.

45. Sachs, N.; de Ligt, J.; Kopper, O.; Gogola, E.; Bounova, G.; Weeber, F.; Balgobind, A.V.; Wind, K.; Gracanin, A.; Begthel, H.; et al. A living biobank of breast cancer organoids captures disease heterogeneity. Cell 2018, 172, 373-386.

46. Tiriac, H.; Belleau, P.; Engle, D.D.; Plenker, D.; Deschênes, A.; Somerville, T.; Froeling, F.E.M.; Burkhart, R.A.; Denroche, R.E.; Jang, G.-H.; et al. Organoid profiling identifies common responders to chemotherapy in pancreatic cancer. Cancer Discov. 2018, 8, 1112-1129.

47. Kopper, O.; de Witte, C.J.; Lohmussaar, K.; Valle-Inclan, J.E.; Hami, N.; Kester, L.; Balgobind, A.V.; Korving, J.; Proost, N.; Begthel, H.; et al. An organoid platform for ovarian cancer captures intra- and interpatient heterogeneity. Nat. Med. 2019, 25, 838-849.

48. Vlachogiannis, G.; Hedayat, S.; Vatsiou, A.; Jamin, Y.; Fernández-Mateos, J.; Khan, K.; Lampis, A.; Eason, K.; Huntingford, I.; Burke, R.; et al. Patient-derived organoids model treatment response of metastatic gastrointestinal cancers. Science (80-. ). 2018, 926, 920-926.

49. Broutier, L.; Mastrogiovanni, G.; Verstegen, M.M.; Francies, H.E.; Gavarró, L.M.; Bradshaw, C.R.; Allen, G.E.; Arnes-Benito, R.; Sidorova, O.; Gaspersz, M.P.; et al. Human primary liver cancer-derived organoid cultures for disease modeling and drug screening. Nat. Med. 2017, 23, $1424-1435$

50. Yan, H.H.N.; Siu, H.C.; Law, S.; Ho, S.L.; Yue, S.S.K.; Tsui, W.Y.; Chan, D.; Chan, A.S.; Ma, S.; Lam, K.O.; et al. A comprehensive human gastric cancer organoid biobank captures tumor subtype heterogeneity and enables therapeutic screening. Cell Stem Cell 2018, 23, 882-897.

51. Ganesh, K.; Wu, C.; O’Rourke, K.P.; Adileh, M.; Szeglin, B.C.; Wasserman, I.; Marco, M.R.; Shady, M.; Zheng, Y.; Karthaus, W.R.; et al. A rectal cancer model establishes a platform to study individual responses to chemoradiation. bioRxiv 2019, 640193.

52. Lee, S.H.; Hu, W.; Matulay, J.T.; Silva, M. V.; Owczarek, T.B.; Kim, K.; Chua, C.W.; Barlow, L.M.J.; Kandoth, C.; Williams, A.B.; et al. Tumor evolution and drug response in patient-derived organoid models of bladder cancer. Cell 2018, 173, 515-528.

53. Dekkers, J.F.; Wiegerinck, C.L.; de Jonge, H.R.; Bronsveld, I.; Janssens, H.M.; de Winter-de Groot, K.M.; Brandsma, A.M.; de Jong, N.W.M.; Bijvelds, M.J.C.; Scholte, B.J.; et al. A functional CFTR assay using primary cystic fibrosis intestinal organoids. Nat. Med. 2013, 19, 939-945.

54. Berkers, G.; van Mourik, P.; Vonk, A.M.; Kruisselbrink, E.; Dekkers, J.F.; de Winter-de Groot, K.M.; Arets, H.G.M.; Marck-van der Wilt, R.E.P.; Dijkema, J.S.; Vanderschuren, M.M.; et al. Rectal organoids enable personalized treatment of cystic fibrosis. Cell Rep. 2019, 26, 1701-1708. 


\section{Supplementary Material}

(a)

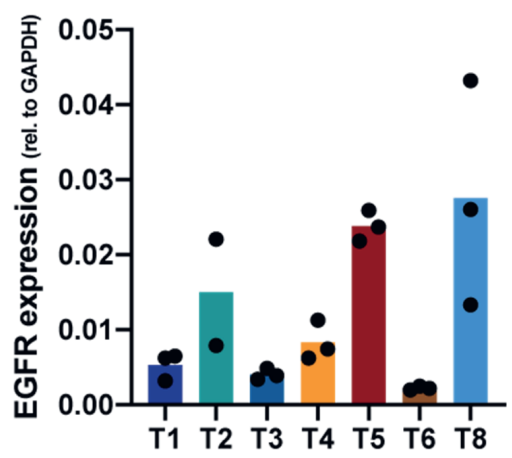

(b)

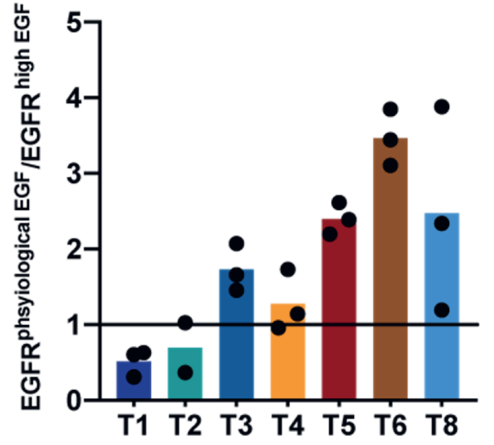

Figure S1 EGFR mRNA expression differs between patient-derived organoid lines and is increased upon medium change to physiological EGF levels. (a), EGFR mRNA expression levels measured in organoid lines derived from different HNSCC patients. Expression is calculated relative to housekeeping gene GAPDH. Experiment was performed in technical triplicate. (b), culturing organoids under physiological EGF resulted in an increase in EGFR mRNA levels in 4/7 lines. EGFR expression is depicted as the ratio of EGFR in medium containing physiological EGF levels, versus EGFR expression in organoid cultured in high EGF medium. When this ratio is $>1$, EGFR expression is increased in response to physiological EGF levels. 

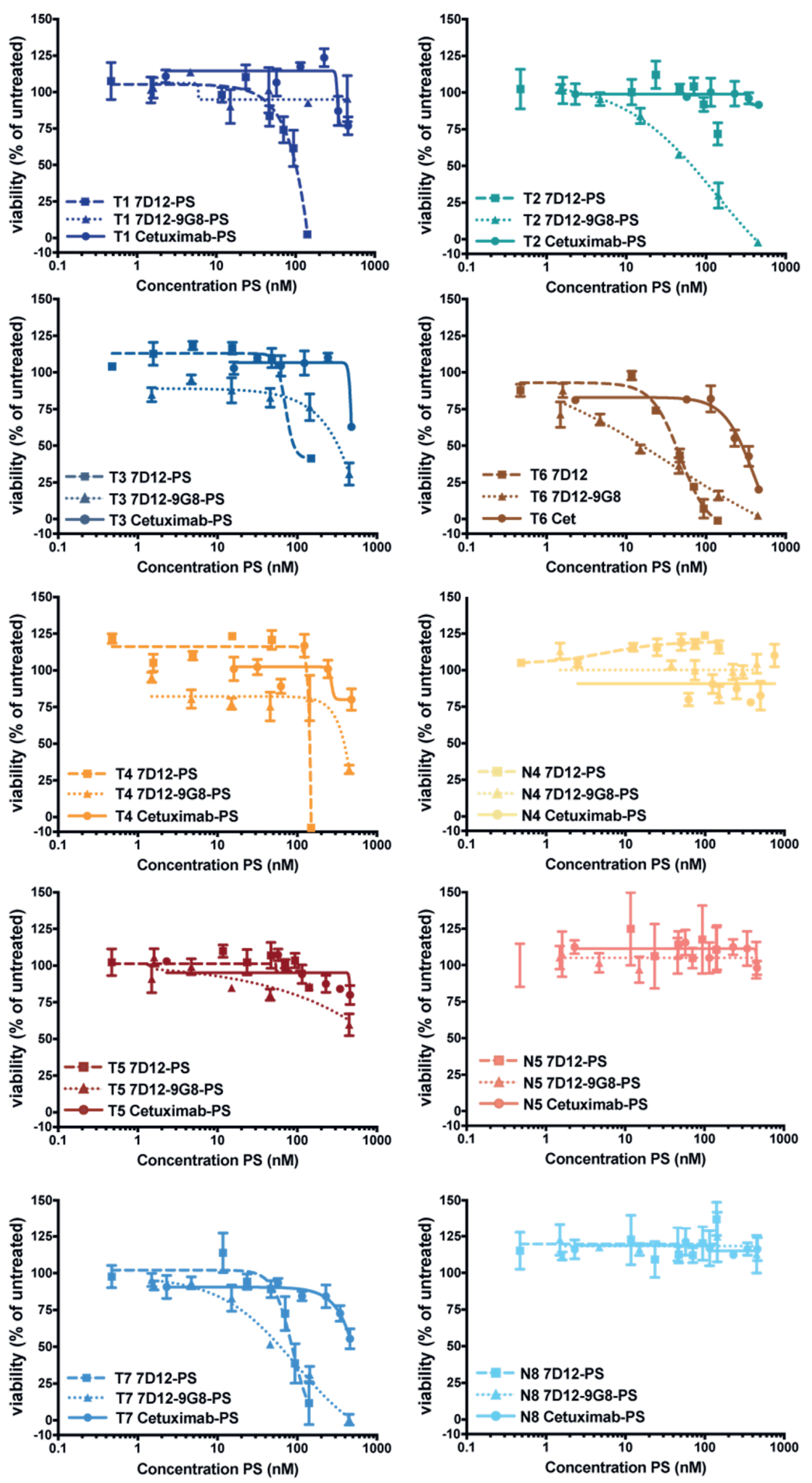
Figure S2 Comparison of different PS carriers in EGFR-targeted PDT. Results of in vitro PDT screens performed with 7D12-PS, 7D12-G98-PS and cetuximab-PS in eight patient-derived HNSCC organoid lines and three matching wildtype organoid lines. Results of 7D12-PS are depicted in dashed lines and squared symbols, results of 7D12-9G8-PS in dotted lines and triangular symbols, and cetuximab-PS in solid lines and circular symbols. Color coding of the different samples is identical to that applied in the main figures.

Supplementary Table S1 Details on targeted sequencing of organoids Used assay: Ion AmpliSeqTM Cancer Hotspot Panel v2+ Next Generation Sequencing Sequencing performed for research purposes in the UMC Utrecht

*this assay only contains hotspot regions and does not provide information on the full sequence of the depicted genes

**this assay has not officially been validated for detecting amplifications

\begin{tabular}{|l|l|}
\hline gene & exons checked by oncopanel \\
\hline ABL1 & $4-7$ \\
\hline AKT1 & 3,6 \\
\hline ALK & $22-25$ \\
\hline APC & 14 \\
\hline ARAF & 6 \\
\hline ATM & $8,9,12,17,26,34,35,36,39,50,54,55,56,59,61,63$ \\
\hline BRAF & 11,15 \\
\hline CALR & 9 \\
\hline CDH1 & $3,8,9$ \\
\hline CDKN2A & 2 \\
\hline CRAF & 6 \\
\hline SDF1R & 7,22 \\
\hline CTNNB1 & 3 \\
\hline EGFR & $3,7,15,18-21$ \\
\hline ERBB2 & $19-24$ \\
\hline ERBB 4 & $3-5,7-9,15,23$ \\
\hline EZH2 & 16 \\
\hline FBXW7 & $5,8-11$ \\
\hline FGFR1 & 4,7 \\
\hline FGFR2 & $5,7,10$ \\
\hline FGFR3 & $7,9,14,16,18$ \\
\hline GNA11 & 5 \\
\hline GNAS & 8,9 \\
\hline GNAQ & 5 \\
\hline HNF1A & 3,4 \\
\hline HRAS & 2,3 \\
\hline IDH1 & 4 \\
\hline & \\
\hline
\end{tabular}

\begin{tabular}{|l|l|}
\hline gene & exons checked by oncopanel \\
\hline IDH2 & 4 \\
\hline JAK2 & 14 \\
\hline JAK3 & $4,13,16$ \\
\hline KDR & $6,7,11,19,21,26,30$ \\
\hline KIT & $2,9-11,13-15,17,18$ \\
\hline KRAS & $2-4$ \\
\hline MDM2 & $6-9$ \\
\hline MET & $2,11,14,16,19$ \\
\hline MLH1 & 12 \\
\hline MPL & 10 \\
\hline MYD88 & 5 \\
\hline NOTCH1 & $25,27,37$ \\
\hline NPM1 & 11 \\
\hline NRAS & $2-4$ \\
\hline PDGFRA & $12,14,15,18$ \\
\hline PIK3CA & $1,4,6,7,9,13,18,20$ \\
\hline PTEN & $3,5-8$ \\
\hline PTPN11 & 3,13 \\
\hline RB1 & $4,6,10,11,14,17$ \\
\hline RET & $10,11,13,15,16$ \\
\hline SMAD4 & $3-6,8-12$ \\
\hline MARCB1 & $2,4,5$ \\
\hline SMO & $3,5,6,9,11$ \\
\hline SRC & 14 \\
\hline STK11 & $1,4,6,8$ \\
\hline TP53 & $2-10$ \\
\hline VHL & $1-3$ \\
\hline & \\
\hline
\end{tabular}





\section{CHAPTER 4}

\section{Molecular targets for anticancer therapies in companion animals and humans: what can we learn from each other?}

Irati Beltrán Hernández ${ }^{1,2,}{ }^{*}$, Jannes Kromhout ${ }^{2,}{ }^{*}$, Erik Teske ${ }^{3}$, Wim E. Hennink ${ }^{1}$, Sebastiaan A. van Nimwegen ${ }^{3}$ and Sabrina Oliveira ${ }^{1,2}$

\footnotetext{
${ }^{1}$ Pharmaceutics, Department of Pharmaceutical Sciences, Faculty of Science, Utrecht University, Utrecht, the Netherlands

${ }^{2}$ Cell Biology, Neurobiology and Biophysics, Department of Biology, Faculty of Science, Utrecht University, Utrecht, the Netherlands

${ }^{3}$ Department of Clinical Sciences, Faculty of Veterinary Medicine, Utrecht University, Utrecht, the Netherlands

* These authors contributed equally to this work
}

Theranostics 2021, in press 


\begin{abstract}
Despite clinical successes in the treatment of some early stage cancers, it is undeniable that novel and innovative approaches are needed to aid in the fight against cancer. Targeted therapies offer the desirable feature of tumor specificity while sparing healthy tissues, thereby minimizing side effects. However, the success rate of translation of these therapies from the preclinical setting to the clinic is dramatically low, highlighting an important point of necessary improvement in the drug development process in the oncology field. The practice of a comparative oncology approach can address some of the current issues, by introducing companion animals with spontaneous tumors in the linear drug development programs. In this way, animals from the veterinary clinic get access to novel/innovative therapies, otherwise inaccessible, while generating robust data to aid therapy refinement and increase translational success. In this review, we present an overview of targetable membrane proteins expressed in the most well-characterized canine and feline solid cancers, greatly resembling the counterpart human malignancies. We identified particular areas in which a closer collaboration between the human and veterinary clinic would benefit both human and veterinary patients. Considerations and challenges to implement comparative oncology in the development of anticancer targeted therapies are also discussed.
\end{abstract}




\section{Introduction}

Cancer is an important cause of death in humans worldwide, accountable for approximately 10 million deaths every year [1]. The use of surgery, chemo- and radiotherapy has certainly led to remarkable clinical outcomes for some early stage cancers [2], but the overall need for improved outcome is undeniable. Differences in anatomical location, aetiology, and molecular biology of cancers stress the necessity to develop novel therapies and combined approaches that more specifically address the cancer in an individual patient. Expanding the therapeutic arsenal to fight cancer, numerous targeted therapies and immunotherapies have emerged in the last decade $[3,4]$. In particular, continuous development in the field of molecular biology and oncogenes raises opportunities for new molecular targeted therapies, e.g. tyrosine kinase inhibitors (TKI), monoclonal antibodies and antibody-drug conjugates targeting cancer cells. These offer selectivity towards cancer cells over normal cells by exploiting genetic alterations in malignant cells, minimizing damage to healthy tissues and improving patient's quality of life [4]. This attractive feature unique to targeted therapies warrants the innovative efforts of researchers to develop new targeted treatments.

In the oncology field, the success rate of translation of a therapy from preclinical to clinical use is as low as $3.4 \%[5,6]$. This is the lowest success rate among therapeutic disciplines and can be partly explained by the use of (immunocompromised) rodent xenograft models as the main experimental tool in the field, which certainly fail to reproduce most aspects of such an intricate disease as cancer. These models, in addition to the obvious biological differences between species, often miss the tissue-specific environment, host immunity, the capacity to metastasize and development of treatment resistance [6]. It is clear that a more clinically relevant setting is needed to bridge the currently existing gap and lead to more successful therapies. Comparative oncology offers this, being a discipline that integrates the study of spontaneous cancers in companion animals into studies of human oncology. Consequently, a win-win scenario is created in which therapeutic options can arise for both humans and veterinary patients. This field of study exemplifies the concept of "One Medicine", which recognises the unity of human and veterinary medicine and that both can advance hand in hand [7]. Dogs and cats with naturally occurring tumors and intact immune systems are of extraordinary translational significance [8]. These veterinary patients present a larger genetic variation than inbred mouse strains and are exposed to environmental factors similar to humans, resulting in less clear-cut results during clinical evaluation that better reflect the human situation. In addition, the shorter life span and lack of therapeutic options for companion animals allow the smooth initiation of therapeutic trials and rapid data collection, while still offering a relatively long-term follow-up [8]. Many cancer types in the veterinary clinic are considered as exceptional models of the human counterpart, exhibiting genetic, molecular and clinical similarities $[9,10]$. With the revealing of the dog genome in 2005 and, more recently, the cat genome in 2014, the study of spontaneous cancers in these animals has become even more interesting for comparative oncology [11,12].

In the context of developing novel targeted therapies, the treatment of veterinary patients with spontaneous tumors can generate robust data and give very valuable insights for treatment refinement, thereby increasing the translational success rate. At the same time, these animals get access to novel and promising therapies, which would otherwise remain inaccessible for these patients (Figure 1). 


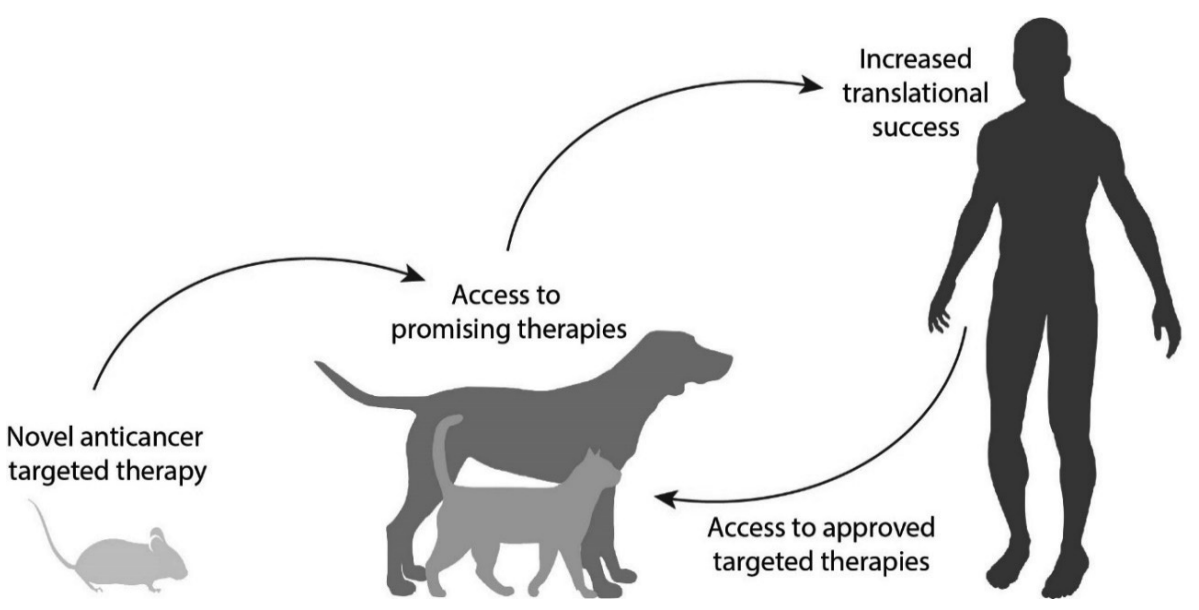

Figure 1 Proposed approach for the development of anticancer targeted therapies. First, encouraging results are obtained in preclinical studies (e.g. rodents) for a targeted therapy utilizing a particular target. The therapy can be evaluated for the treatment of relevant cancers in companion animals expressing the target in question, allowing access to novel and promising therapeutic approaches for these veterinary patients. Robust data are thus acquired in a more relevant setting, to guide the translation of the therapy towards the equivalent human cancer while increasing translational success. At the same time, the use of current successful targeted therapies in the human clinic can be expanded to the veterinary clinic, based on the knowledge of which targets are present in the different canine and feline cancers.

In this review, we present an overview of molecular targets in companion animals and their expression in relevant cancers (i.e. with translation potential). The focus is on targetable membrane proteins with high homology across species present on the most well-characterized solid cancer models: canine and feline oral squamous cell carcinoma, canine bladder cancer, canine and feline mammary carcinoma, canine osteosarcoma and canine melanoma. A brief introduction of each cancer, its human counterpart and available targeted therapies is given in Table 1, together with a collection of comparative studies highlighting the rationale to focus on the selected cancer types. This table evidences the existing gap between human and veterinary medicine, since no targeted therapies are yet approved for the reviewed cancer types in companion animals. From the listed targeted therapies in Table 1, only the approved monoclonal antibodies cetuximab and trastuzumab are being assessed at the preclinical level for the treatment of the veterinary malignancies, and thus these therapies will be addressed further in this review. 
Table 1 Overview of the translational cancer models included in this review, i.e. human and canine/feline counterpart, available targeted therapies and selected reviews describing similarities

\begin{tabular}{|c|c|c|c|}
\hline Cancer & $\%$ of all cancers & $\begin{array}{l}\text { Approved targeted } \\
\text { therapy (target) }\end{array}$ & $\begin{array}{c}\text { Selected } \\
\text { review }\end{array}$ \\
\hline $\begin{array}{c}\text { Human head and neck } \\
\text { squamous cell carcinoma }\end{array}$ & $6 \%[1]$ & Cetuximab (EGFR) & \multirow[b]{2}{*}[13,14]{} \\
\hline $\begin{array}{l}\text { Canine and feline oral } \\
\text { squamous cell carcinoma }\end{array}$ & $\begin{array}{l}8 \% \text { of all feline cancers }[15] \\
2 \% \text { of all canine cancers }[16]\end{array}$ & - & \\
\hline $\begin{array}{l}\text { Human invasive } \\
\text { transitional cell } \\
\text { carcinoma (TCC) }\end{array}$ & $\begin{array}{c}3 \% \text { are bladder cancer, of } \\
\text { which } 20 \% \text { are invasive TCC } \\
{[1]}\end{array}$ & Erdafitinib (FGFR) & \multirow{2}{*}{ [17] } \\
\hline Canine invasive TCC & $\begin{array}{c}2 \% \text { are bladder cancer, of } \\
\text { which }>90 \% \text { are invasive } \\
\text { TCC }[17]\end{array}$ & - & \\
\hline $\begin{array}{l}\text { Human mammary } \\
\text { carcinoma }\end{array}$ & $12 \%(26 \%$ in women $)[1]$ & $\begin{array}{c}\text { Trastuzumab (HER2), } \\
\text { Bevacizumab (VEGF), } \\
\text { others }\end{array}$ & \multirow[t]{2}{*}[10,18]{} \\
\hline $\begin{array}{l}\text { Canine and feline } \\
\text { mammary carcinoma }\end{array}$ & $\begin{array}{c}25 \% \text { in female dogs [18] } \\
17 \% \text { in female cats [19] }\end{array}$ & - & \\
\hline Human osteosarcoma & $\begin{array}{c}3.5 \% \text { of all childhood cancers } \\
<1 \% \text { in adults }[20]\end{array}$ & $\begin{array}{l}\text { Sorafenib (multiple } \\
\text { kinases) }\end{array}$ & \multirow[b]{2}{*}[21]{} \\
\hline Canine osteosarcoma & $\begin{array}{c}2 \% \text { (especially large size } \\
\text { breeds) }[22]\end{array}$ & - & \\
\hline $\begin{array}{l}\text { Human melanoma } \\
\text { (non-UV induced) }\end{array}$ & $\begin{array}{c}6 \% \text { are melanoma, of which } \\
15 \% \text { are non-UV induced } \\
{[23]}\end{array}$ & $\begin{array}{c}\text { Vemurafenib (BRAF), } \\
\text { Trametinib (MEK), } \\
\text { others }\end{array}$ & \multirow{2}{*}{ [24] } \\
\hline Canine melanoma & $\begin{array}{c}7 \% \text { are melanoma, of which } \\
>95 \% \text { are non-UV induced } \\
{[25]}\end{array}$ & - & \\
\hline
\end{tabular}

EGFR: epidermal growth factor receptor; FGFR: fibroblast growth factor receptor; HER2: human epidermal growth factor receptor 2; VEGF: vascular endothelial growth factor; BRAF: B-Raf proto-oncogene; MEK: mitogen-activated protein kinase.

The different membrane targets that are addressed in this review are presented in Table 2 , emphasizing their relevance in the oncology field. Most of these proteins are tyrosine kinase receptors mediating cell proliferation and survival. G protein-coupled receptors and nutrient transporters are also common targets under evaluation in human clinical trials. Table 2 lists some examples of the therapies approved for human use, mostly TKIs (e.g. gefitinib and lapatinib) and the already mentioned monoclonal antibodies (i.e. cetuximab and trastuzumab). Next to these, also other therapies are indicated in Table 2, 
though only those that were found to be tested in companion animals are described further.

Knowing the targets overexpressed in these relevant animal cancers, not only serves to guide the evaluation of targeted therapies in clinically relevant models of the human disease, but also to expand the use of current successful targeted therapies in humans towards the veterinary clinic. The feasibility of the proposed approach is supported by the fact that cancer remains a leading cause of death not only in humans, but also in companion animals. To compare, dogs develop cancer at a similar rate as humans ( 1 in 3 ), while cats are less affected ( 1 in 4 to 5) [26]. About $50 \%$ of dogs and $30 \%$ of cats over age ten die from cancer. Most of the discussed animal cancers are often compared to adult human cancers, since those are rare during childhood and have a higher incidence in geriatric animals, while canine osteosarcoma is considered to closely resemble the pediatric cancer [27] 


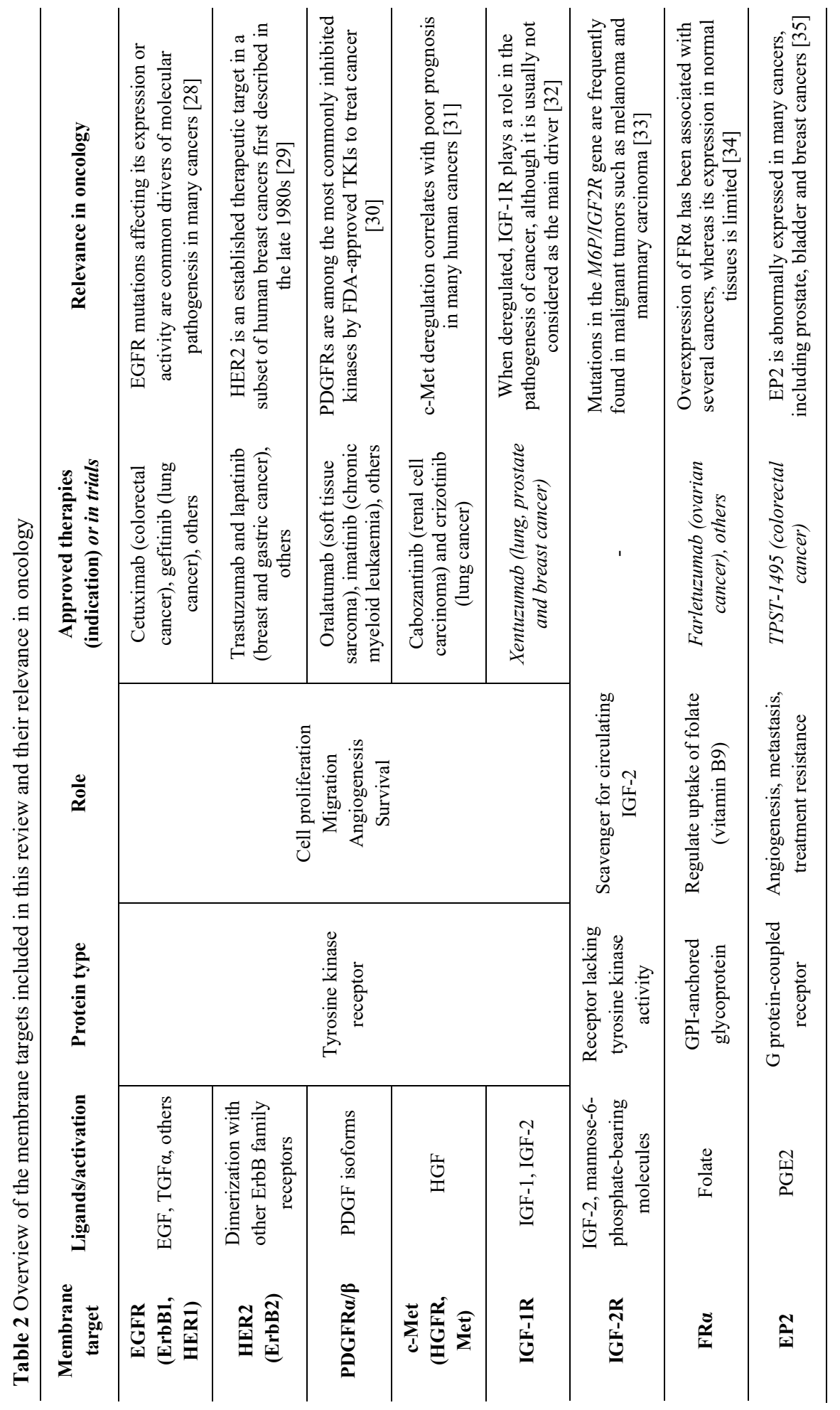




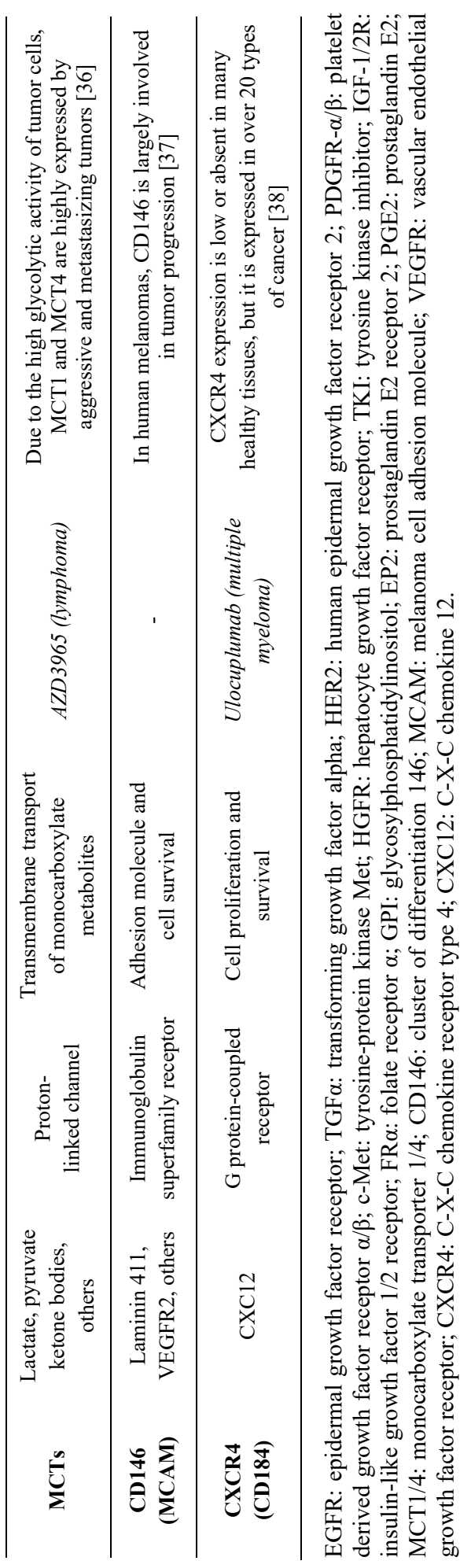




\section{Epidermal growth factor receptor}

\section{Head and neck squamous cell carcinoma}

EGFR overexpression has been reported in up to $90 \%$ of human head and neck squamous cell carcinomas (HNSCC) and also in feline oral squamous cell carcinoma (OSCC), making it an interesting therapeutic target in both species [39-41]. Although several EGFR-targeting small molecule inhibitors and monoclonal antibodies are currently approved and under clinical evaluation for the treatment of human HNSCC $[42,43]$, clinical evaluation of agents targeting EGFR in feline OSCC has not been reported. In vitro studies with a feline OSCC cell line have been conducted to target EGFR with the TKI gefitinib and RNA interference $[44,45]$. While gefitinib caused a reduction in cell proliferation, this was accomplished at a relatively high dose and gefitinib resistance developed. By combining siRNA with gefitinib treatment, it was possible to surpass the acquired resistance. Interestingly, the combination of siRNA targeting of EGFR and radiation therapy showed an additive cytotoxic effect in the feline cell line [44]. These results, although scarce and in an initial stage, support the notion of EGFR targeting to treat feline OSCC.

\section{Invasive transitional cell carcinoma}

EGFR is overexpressed in both human and canine invasive transitional cell carcinoma (TCC) in about $75 \%$ of the cases $[17,46,47]$. Several EGFR-targeting compounds have been or are currently evaluated in clinical trials of human TCC, but so far with mixed results [17]. Recently, a particular 67-gene signature has been associated with sensitivity to EGFR inhibition in human bladder cancer cell lines [48]. Regarding canine invasive TCC, there is only one clinical study involving EGFR-targeting as therapy [49]. In this study, an EGF-conjugated anthrax toxin was used to target EGFR-expressing human and canine TCC cell lines. After encouraging in vitro results, the effects of the EGF-toxin were evaluated in 6 dogs with invasive TCC, resulting in a $30 \%$ reduction of the tumor size in all dogs. These data show promising effects and follow-up studies are warranted. In another recent study, near-infrared photoimmunotherapy was used to target EGFR in canine invasive TCC cell lines [50]. The EGFR-targeting monoclonal antibody can225IgG (caninized cetuximab) was conjugated to a photosensitizer (IRDye700DX) and tested in canine TCC cell lines, showing specificity and cytotoxic potency. Using a xenograft mice model of canine invasive TCC, NIT-PIT treatment led to a statistically significant inhibition of tumor growth and prolonged survival, highlighting thus the promise of the approach.

\section{Osteosarcoma}

EGFR is frequently overexpressed in human osteosarcoma (OSA); however, the exact contribution of EGFR in OSA prognosis and development is still not fully understood [51,52]. EGFR is expressed in canine OSA cell lines and tissues, and a higher expression correlates with a poor prognosis [53,54]. Currently, several ongoing clinical trials evaluate the use of EGFR-targeting agents in human patients with OSA [52]. As for canine OSA, only in vitro data are available so far [54-56]. Three small molecule EGFRtargeting TKIs, i.e. erbstatin analogue, erlotinib and gefitinib, have been tested alone or in combination with different therapeutic approaches. The three drugs showed activity as monotherapy, but combinations yielded better results. In particular, erbstatin analogue was tested in combination with the chemotherapy agent doxorubicin, resulting in 
increased cell death compared to erbstatin monotherapy. Erlotinib was tested in combination with radiation therapy and enhanced sensitivity to radiation was observed. Lastly, the combination of gefitinib and the c-Met inhibitor crizotinib showed an additive effect on cell proliferation inhibition. Altogether, combination strategies involving EGFR targeting show potential for the treatment of canine OSA.

\section{Mammary carcinoma}

EGFR expression is found in $15-45 \%$ of human breast cancer tissues [57]. Accordingly, the EGFR TKIs gefitinib, erlotinib, lapatinib and afatinib are approved for the treatment of some subsets of breast cancer, while the evaluation of more EGFR-targeting drugs is ongoing in clinical trials [58]. In canine mammary carcinoma (CMC) tissues, similar EGFR expression as in the human equivalent cancer has been reported [59]. Similarly, EGFR was found to be significantly overexpressed in cell lines, tissues and xenograft mouse models of feline mammary carcinoma (FMC) [60]. Several studies report cell proliferation inhibition induced by EGFR-targeting in CMC cell lines. Cetuximab and its caninized version (can225IgG), gefitinib and EGFR siRNA have all inhibited cell proliferation as a single agent [61-63]. Only in one of these studies a FMC cell line was included, also showing cell proliferation inhibition after gefitinib treatment [62].

\section{Melanoma}

EGFR has also been of interest as a therapeutic target in human melanomas, and it can play a role in the progression of cutaneous melanomas [64]. Two clinical phase II trials have been conducted with EGFR-targeting agents, but the outcomes were poor in human patients with metastatic melanomas $[65,66]$. Therefore, there are currently no EGFRtargeted therapies approved for human melanomas. In canine melanoma, little is known regarding EGFR expression and its role. One recent study reported the expression of EGFR and other receptors from the EGFR family in canine melanomas, concluding that EGFR overexpression occurs only in $5 \%$ of the oral melanomas and $16 \%$ of the skin melanomas [67]. In addition, an increased proliferative index was associated with a decreased EGFR expression in the skin melanomas. In another recent study, the expression of EGFR was found downregulated in oral melanoma compared to the healthy oral tissue [68]. Therefore, EGFR does not seem to be a promising target in human and canine melanoma, but targeting of this receptor in the canine disease has not been investigated yet.

\section{Human epidermal growth factor receptor 2}

\section{Invasive transitional cell carcinoma}

HER2 overexpression is common in human TCC and it is, therefore, an interesting target [69]. Likewise, significant overexpression of HER2 compared to normal canine bladder has been described in canine invasive TCC tissues at both the RNA and protein level [70 72]. While multiple clinical trials are ongoing to evaluate different HER2-targeting drugs in human patients with TCC [73], the targeting of this protein has not been yet studied in the context of canine TCC. The outcome of the current human clinical trials will certainly shed light on the value of targeting HER2 in this type of bladder cancer and may spur research also in the field of veterinary oncology. 


\section{Osteosarcoma}

The role of HER2 in human OSA has been controversial. Multiple studies report overexpression of HER2, while others describe the opposite [74-76]. As for canine OSA, overexpression of HER2 has been detected in different OSA cell lines and tissues at the RNA level [77]. Despite the controversial findings on HER2 in human OSA, a clinical trial has been conducted with trastuzumab, a HER2-targeting monoclonal antibody, in combination with chemotherapy [78]. The outcome was rather disappointing and HER2 positive patients did not have a significant therapeutic benefit. Nevertheless, HER2 seems to remain an interesting target in these cancers for targeted therapy and, particularly, for immunotherapy [52]. In human patients with HER2-positive OSA, HER2-specific chimeric antigen receptor $T$ cell therapy has shown initial promising results [79]. Furthermore, dogs with HER2-positive OSA were successfully treated with a vaccine consisting of recombinant Listeria bacteria expressing a chimeric human HER2, which induced HER2-specific immunity and reduced the incidence of metastasis [80]. Altogether, it is reasonable to consider immunotherapies involving HER2 targeting as a therapeutic option for both human and canine OSA, but the targeting of this receptor alone has not yet shown promise.

\section{Mammary carcinoma}

HER2 is a major marker for human breast cancer classification and its presence or absence mainly guides the choice of therapeutic strategy. HER2 overexpression is found in $10-40 \%$ of human breast cancers [81]. For the treatment of HER2-positive breast cancer, multiple HER2-targeting agents are already approved, e.g. trastuzumab, pertuzumab and afatinib, and others are being tested in ongoing clinical trials [58]. In CMC and FMC, HER2 overexpression is in the same range as in human breast cancers [82-85]. Nonetheless, studies of HER2-targeting in these veterinary patients have been so far limited to in vitro evaluations. The anti-HER2 antibody trastuzumab was able to inhibit cell proliferation in two CMC cell lines [61]. On the other hand, using other CMC and FMC cell lines, the use of the HER2 inhibitor AG825 or a HER2 siRNA only caused a slight inhibition of cell proliferation [62]. All things considered, the genetic, molecular and clinical similarities between the human and canine/feline disease support the value of targeting HER2 in companion animals with mammary carcinoma.

\section{Melanoma}

The role of HER2 in human melanomas is still under debate, because of contradictory results regarding the overexpression of this target [86-88]. In canine oral and skin melanomas, the role of HER2 is still largely unknown and there is only one study addressing this. HER 2 membranous expression was found in 50 and $43 \%$ of the oral and skin melanomas, respectively, but this was not considered to be overexpressed [67]. HER2 expression in the oral melanomas was associated with tumor progression, because of its correlation with emboli occurrence. So far, no solid conclusion can be drawn regarding the potential of HER2 targeting to treat melanoma.

\section{Platelet-derived growth factor receptors}

\section{Osteosarcoma}

Both PDGFR $\alpha$ and PDGFR $\beta$ are frequently overexpressed in OSA human tissues $[89,90]$. In canine OSA tissue, the expression of both proteins is commonly found and, in canine 
OSA cell lines, overexpression of the two receptors has been reported at the RNA level [91]. However, clinical trials evaluating different PDGFR-targeted therapies in human OSA have not been successful. As a result, it has been suggested to target PDGFRs not as monotherapy, but rather in combination with other therapeutic agents such as check point inhibitors or chemotherapy [90]. There are no studies available in which the targeting of PDGFRs in canine OSA has been investigated, but combination strategies might be the answer as in the case of the human disease.

\section{Melanoma}

In human cutaneous melanomas, overexpression of PDGFR $\alpha$ is associated with decreased cell proliferation, whereas the role of PDGFR $\beta$ in melanomas is, on the other hand, less clear $[92,93]$. Still, several phase II and III clinical trials are being conducted in patients with malignant melanomas evaluating TKIs that target PDGFRs amongst other tyrosine kinase receptors [94]. In canine oral melanomas, the co-expression of PDGFR $\alpha$ and PDGFR $\beta$ has been described in $37.5 \%$ of the cases, which corresponded with a significant shorter overall survival compared to dogs without co-expression [95]. Although the targeting of exclusively PDGFRs has not been investigated yet in companion animals with melanoma, the encouraging results from human clinical trials may instigate researchers in this direction.

\section{Hepatocyte growth factor receptor}

\section{Osteosarcoma}

c-Met is frequently found overexpressed in human and canine OSA tissues and cell lines [54,96-98]. Nevertheless, to date, there are no clinical data available of c-Met-targeting agents to treat OSA. The two approved multi-TKI cabozantinib and crizotinib, which target c-Met amongst others, have been tested in human OSA cell lines and xenograft mouse models with promising results $[99,100]$. Liao et al. tested a c-Met-targeting drug called PF2362376 in canine OSA cell lines, which inhibited c-Met and induced cell death at a high dose [101]. Another drug that targets c-Met, called PHA-665752, and a c-Met shRNA have also been evaluated as single agents in two canine OSA cell lines. Although the two agents did not affect cell proliferation, the motility of the cell lines was decreased [98]. Although not clear yet whether targeting c-Met alone can be of value, there are indications that c-Met inhibition can be a promising strategy to treat OSA when also other oncogenic pathways are being targeted.

\section{Insulin-like growth factor-1 receptor}

\section{Osteosarcoma}

The role of IGF-1 and IGF-1R has been investigated in several human cancers, including OSA [32]. A significant overexpression of IGF-1R has been found in human OSA tissues compared to healthy bone tissue [102]. In canine OSA cell lines and tissues, high expression of IGF-1R is frequently observed at both the RNA and protein level $[103,104]$. One clinical study assessing the efficacy of an IGF-1R-targeting drug has shown poor results in humans with OSA [105]. Nonetheless, more IGF-1R-targeting drugs are being investigated in clinical trials to treat human OSA [106], while no data are available 
concerning canine OSA. The results of the ongoing human clinical trials, when promising, may guide the use of similar therapeutic strategies in dogs with OSA.

\section{Mammary carcinoma}

Expression of IGF-1R is frequently found in human breast cancer with varying percentages in the different cancer subtypes [107]. Therefore, IGF-1R has been an interesting target in breast cancer and multiple clinical trials have taken place with IGF$1 \mathrm{R}$-targeting agents [108]. However, these clinical trials showed poor outcomes, resulting in no approved IGF-1R-targeting drugs in human breast cancer so far. In multiple studies with tissue samples of canine mammary gland carcinomas and invasive $\mathrm{CMC}$, high expression of IGF-1R was commonly found, but contradictory findings have been reported regarding its correlation with prognosis in CMC [109-111]. The role and expression of IGF-1R has not been studied in FMC, and no therapeutic strategies targeting this receptor have been assessed in CMC or FMC. Clearly, while human data discourages IGF-1R targeting in breast cancer, more research is needed to get a better understanding of the potential of this approach in companion animals with mammary carcinoma.

\section{Melanoma}

IGF-1R often plays a role in the different types of human melanomas and, therefore, it has been considered an interesting drug target for this disease [112,113]. Among multiple tyrosine kinase receptors, IGF-1R has been found to be the most abundant in canine malignant melanoma cell lines, and its expression was subsequently confirmed in the majority of canine melanoma tissues [114]. There are several preclinical studies showing promising effects of IGF-1R targeting in combination with other therapeutic agents for the treatment of human melanoma [115]. Nevertheless, the use of IGF-1R-targeting agents has not been evaluated in canine melanomas, neither in vitro nor in vivo. It is reasonable to consider IGF-1R a good target in canine melanoma, based on its high expression and the so far encouraging results in human melanoma.

\section{Insulin-like growth factor-2 receptor}

\section{Osteosarcoma}

In contrast to IGF-1R, research conducted with IGF-2R as a therapeutic target is still in the initial phases. In human primary OSA cell lines, IGF-2R has been found to be significantly overexpressed [96]. Monoclonal antibodies targeting IGF-2R conjugated to different cytotoxic radioisotopes, for radioimmunotherapy, have been evaluated in human OSA cell lines as well as patient-derived xenograft mouse models [116,117]. The authors showed specificity of the radiolabelled antibody and the ability to supress tumor growth. Furthermore, the same authors described IGF-2R expression in canine OSA tissue, making it an interesting target for further research [116].

\section{Folate receptor $\alpha$}

\section{Invasive transitional cell carcinoma}

Dhawan et al. investigated the expression of FRs in human and canine invasive TCC tissues, which was found in 78 and $76 \%$ of the cases, respectively [118]. In the same study, the FR-targeting drug folate-vinblastine conjugate was intravenously administered 
to treat 9 dogs with FR-positive invasive TCC. The drug led to partial remission in $56 \%$ of the dogs and stable disease in $44 \%$. The same research group continued this line of investigation and treated 28 dogs with invasive TCC with a different FR-targeting drug, i.e. folate-tubulysin conjugate. This time, clinical benefit was observed in $71 \%$ of the dogs, including partial remission in $11 \%$ and stable disease in $60 \%$ [119]. The encouraging results justify the assessment of FR-targeting agents to treat invasive TCC in the human clinic as well.

\section{Prostaglandin E2 receptor 2}

\section{Invasive transitional cell carcinoma}

The expression levels of EP2 in human urothelial cancer tissues have been shown to be significantly upregulated compared to normal urothelium [120]. Interestingly, a decreased EP2 expression in the cytoplasm and nucleus in bladder cancer tissues compared to normal bladder has also been reported [121]. When comparing the gene expression patterns of invasive canine TCC tissues and normal bladder, the gene encoding for EP2, PTGER2, was the most upregulated gene in the TCC samples [72]. After that finding, EP2 protein expression was detected in the tumor cells of 11 out of 15 canine TCC tissues, whereas EP2 was absent in normal epithelial cells. Altogether, the overexpression of EP2 in human and canine TCC makes it a promising target, but the efficacy of targeting agents has yet to be investigated.

\section{Monocarboxylate transporters}

\section{Head and neck squamous cell carcinoma}

Increased expression of MCT1 and MCT4 in human oral SCC has been correlated with a poor survival prognosis and, therefore, MCT1 and MCT4 were proposed as therapeutic targets in HNSCC $[122,123]$. More recently, high expression of MCT1 in feline OSCC cell lines and tissues was reported, whereas MCT4 expression was relatively low [124]. Accordingly, the action of a dual MCT1- and MCT4-inhibiting drug, MD-1, was tested in feline OSCC and human HNSCC cell lines as well as in an orthoptic feline OSCC xenograft mouse model. The targeting agent induced cell death in the human and feline cell lines and inhibited tumor growth in the orthoptic mouse model, highlighting the promise of MCTs as therapeutic targets in both species.

\section{CD146}

\section{Melanoma}

CD146 is highly expressed in human primary and metastatic melanomas and is a marker of poor prognosis in these patients [37]. Multiple antibodies have been developed to target human CD146 and tested in preclinical settings with promising results $[37,125,126]$. In canine tissues of oral and skin melanoma, CD146 is frequently highly expressed as well, especially in oral melanomas [127,128]. However, at present no studies have been published that investigated CD146 targeting in canine melanoma. Clinical trials to be conducted in the near future will give insights into the value of CD146 as a therapeutic target in the clinical setting. 


\section{C-X-C chemokine receptor type 4}

\section{Mammary carcinoma}

Targeting CXCR4 in human breast cancer patients is currently under evaluation in clinical trials [129]. In FMC tissues, CXCR4 is frequently highly expressed, whereas CXCR4 expression is not detected in healthy mammary tissues of cats $[130,131]$. Moreover, CXCR4 has been described as one of the top upstream regulators in canine mammary tumors [132]. Further research in companion animals may be encouraged by the outcome of the current clinical studies in human patients [129].

\section{Multi-kinase inhibitors}

Many targetable proteins are tyrosine kinase receptors on the cell membrane. Upon their activation (e.g. ligand binding), intracellular signaling occurs, inducing a multitude of cellular processes, including cell proliferation, differentiation and survival. Alteration in expression and/or activation of tyrosine kinase receptors is common in many human cancers and, therefore, these proteins have been of interest in the field of targeted therapeutics [133].

Sunitinib (Sutent $\left.{ }^{\circledR}\right)$ and toceranib phosphate (Palladia $\left.{ }^{\circledR}\right)$ are two drugs targeting multiple of these tyrosine kinase receptors simultaneously, including the vascular endothelial growth factor receptor (VEGFR), PDGFR, c-Kit, colony stimulating factor 1 receptor and fms-like tyrosine kinase 3 [134-136]. Sunitinib and toceranib phosphate are actually structural analogues, used for the treatment of cancer in the human and veterinary clinic, respectively. Sunitinib has been approved for the treatment of different cancers, such as renal cell carcinoma and gastrointestinal stromal tumors. Toceranib phosphate was the first drug approved for targeted cancer treatment in companion animals, indicated for canine mast cell tumors.

Another TKI with a broad range of targets is dasatinib (Sprycel, Dasanix), which targets several tyrosine kinase receptors such as c-Kit and PDGFRs, as well as the SRC kinase family of non-receptor signaling proteins. This drug has already been approved by the FDA for treatment of two types of human leukaemia [137], and its use in the veterinary clinic is gaining interest. Masitinib mesylate (Kinavet, Masivet) is another multi-TKI mainly inhibiting c-Kit and, to a lesser extent, other tyrosine kinase receptors such as PDGFRs and fibroblast growth factor receptor 3 (FGFR3) [138]. Masitinib is approved for the treatment of canine mast cell tumors, while its evaluation in humans with solid tumors, such as pancreatic and colorectal cancer, is ongoing. An overview of the evaluation of toceranib phosphate, dasatinib and masitinib mesylate in the veterinary clinic (for the cancer models here reviewed) is shown in Table 3.

Table 3 Overview of the use of toceranib phosphate, dasatinib and masitinib mesylate in veterinary clinical studies and in vitro.

\begin{tabular}{c|cccc}
\hline Cancer & Toceranib phosphate & Dasatinib & Masitinib mesylate & Ref. \\
\hline HNSCC & Dogs \& cats & & Canine \& feline cells & {$[134,135,139]$} \\
TCC & Dogs & & & {$[140]$} \\
OSA & Dogs & Dogs & & {$[135,141,142]$} \\
MC & Dogs & & & {$[143,144]$} \\
MM & Dogs & & Dogs & {$[144,145]$} \\
\hline
\end{tabular}

HNSCC: head and neck squamous cell carcinoma; TCC: transitional cell carcinoma; OSA: osteosarcoma; MC: mammary carcinoma; MM: malignant melanoma. 


\section{Head and neck squamous cell carcinoma}

The use of sunitinib has been evaluated in phase II clinical trials in recurrent or metastatic HNSCC, but resulted in poor outcomes due to low efficacy [146,147]. Since then, toceranib phosphate has been tested in different cancers in dogs and cats, including canine and feline OSCC $[134,135,148]$. London et al. treated 8 dogs with carcinoma in the head and neck region with toceranib phosphate, and 6 of these dogs experienced clinical benefit [135]. In a retrospective study of cats with OSCC, toceranib phosphate treatment significantly extended the lifespan compared to untreated cats [134]. Here the treatment was combined with the administration of non-steroidal anti-inflammatory drugs (NSAIDs) and the beneficial effect of toceranib phosphate alone was not demonstrated. Nevertheless, overall, promising results have been observed so far for the use of toceranib phosphate in the treatment of OSCC in companion animals.

The use of masitinib, which has the highest affinity for c-Kit, has been evaluated in feline and canine OSCC cell lines. Overexpression and activation of c-Kit was found in human HNSCC [149], and similar expression levels have been detected in feline and canine OSCC cell lines [139]. Masitinib inhibits the downstream pathway of c-Kit in these cell lines, but also induces expression of cyclooxygenase 2 (COX2), an enzyme involved in cell proliferation. Consequently, co-treatment with piroxicam (a COX2 inhibitor) resulted in the best inhibition of cell proliferation for the different SCC cell lines [139]. Clearly, further research is still needed to elucidate the promise of these combination strategies.

\section{Invasive transitional cell carcinoma}

Sunitinib has been evaluated in several phase II clinical trials of human patients with noninvasive or metastatic TCC. The general outcome of those studies was unsatisfactory, despite clinical benefit in some patients [150-152]. The use of toceranib phosphate has been investigated for the treatment of canine TCC. The expression of PDGFR $\beta$, VEGFR2 and c-Kit, all targets of toceranib phosphate, has been described in canine invasive TCC, although only PDGFR- $\beta$ was found significantly overexpressed in comparison to normal bladder tissue [153]. Owing to these findings, a retrospective study was recently performed regarding toceranib phosphate treatment in dogs with TCC, showing clinical benefit in $86.7 \%$ of the dogs [140]. The study had some limitations, such as the coadministration of NSAIDs which can positively affect overall survival and the presumptive diagnosis of 12 out of 27 dogs. Nonetheless, the results are encouraging for the use of toceranib phosphate to treat canine TCC.

\section{Osteosarcoma}

Sunitinib has shown promising effects in human OSA cell lines and xenograft mouse models [154], but no clinical data are available to date. The use of toceranib phosphate in canine OSA has been evaluated in the last couple of years. First, a retrospective study showed the first preliminary evidence of clinical benefits in canine OSA [135]. However, follow-up studies failed to demonstrate a significant clinical benefit of toceranib phosphate as monotherapy or in combination with chemotherapy or other therapeutic agents [155-158]. In a recent paper by Sánchez-Cespedes et al., toceranib phosphate was investigated in canine OSA cell lines and in two orthotopic xenograft canine OSA mouse models, resulting in decreased tumor growth only in one of the two mouse models [159]. The OSA cell line used for this particular xenograft had high expression of PDGFR, cKit and VEGFR2 and, as a result, it has been suggested that toceranib phosphate is more effective in tumors with high expression of these receptors. 
Dasatinib has been tested in human clinical trials for the treatment of different sarcomas, including OSA, but there was insufficient activity as a single agent [160]. In a single-case study of a dog with OSA, dasatinib was used as a treatment [141]. The treated dog in the study first underwent surgery, by which primary tumor cells were obtained and cultured. Together with other canine OSA cell lines, the primary cells were screened for 86 small molecule inhibitors. Here dasatinib showed the most promising result and, consequently, was used to treat the dog after receiving surgery and chemotherapy. Eight months after completion of the treatment, the tumor had not recurred. In line with these observations, Marley et al. treated 4 dogs with OSA with dasatinib after surgery and chemotherapy, leading to a prolonged survival time and indicating the potential benefit of this drug for the treatment of canine OSA [142].

\section{Mammary carcinoma}

Multiple phase III clinical trials of sunitinib treatment in patients with advanced breast cancer, either as monotherapy or in combination with chemotherapy, have not shown satisfactory results. Therefore, sunitinib has not been approved as treatment in human breast cancer [161-163]. Evaluation of toceranib phosphate in a clinical setting to treat dogs bearing mammary carcinomas has been limited. Five dogs with mammary carcinoma were included and 2 of these had a partial response [144]. Eighteen dogs with inflammatory mammary carcinoma were treated with toceranib phosphate in combination with piroxicam and thalidomide. Of those 18 dogs, 4 received additional radiation therapy, which led to partial response and significant increased survival time compared to the dogs which did not receive radiotherapy. For the 14 dogs not treated with radiation therapy, the combination treatment of toceranib phosphate, piroxicam and thalidomide caused a partial response in 3 dogs and 6 dogs experienced stable disease [143]. Altogether, toceranib phosphate may be effective against canine mammary carcinoma in a multimodal approach, but the effectiveness as a single agent remains to be elucidated.

\section{Melanoma}

Sunitinib has been evaluated in human clinical trials to treat cutaneous, mucosal and uveal melanomas. Nevertheless, sunitinib has shown limited activity in those trials and it has been suggested that sunitinib could be more beneficial in combination with other drugs for the treatment of melanoma [164,165]. In two canine clinical studies evaluating toceranib phosphate, a total of 4 dogs with melanoma were included, from which 3 dogs achieved a stable disease $[144,145]$. Although the first results are encouraging, the benefit of toceranib phosphate to treat canine melanomas needs to be addressed in larger studies.

The use of masitinib mesylate has also been evaluated in dogs with melanoma, strongly driven by the fact that c-Kit is a therapeutic target in human melanomas, especially in advanced melanomas with a mutated c-Kit gene [166]. Different c-Kit inhibitors have already been tested in human clinical trials and imatinib is currently recommended for second-line treatment of c-Kit-mutated melanoma [167]. In canine cutaneous and mucosal melanomas, only a few studies have investigated the expression, mutations and role of c-Kit, and the precise role of c-Kit is still not completely understood in these melanomas [168-171]. In a recent study by Giuliano \& Dobson, masitinib mesylate was evaluated as a treatment in 17 dogs with advanced malignant melanomas. Only 2 dogs with mucosal melanomas had a partial response, while 7 dogs achieved stable disease. The researchers concluded that masitinib mesylate treatment is not efficient, at least as monotherapy, for advanced malignant melanoma in dogs [172]. 


\section{Considerations for the development of targeted therapies in comparative oncology}

The use of targeted therapies to treat human cancers has been significantly more implemented than in the veterinary clinic [173]. Nevertheless, with the latest developments in molecular biology and the veterinary approval of the two multi-TKIs toceranib and masitinib, targeted therapies in veterinary oncology are certainly becoming a more important focus of cancer research. Dogs with spontaneous cancers have been more of interest in comparative oncology than their feline comrades [10,174]. However, for some cancer types, cats can be more relevant for translational research; for instance, as a model for human HNSCC and metastatic breast cancer [10]. Most advances in targeted therapies in the veterinary landscape are directed towards canine lymphomas and mast cell tumors. In this review, we have focused on the translation of targeted therapies for solid tumors, by presenting an overview of molecular targets in human cancers and the counterpart malignancy in companion animals, as well as the outcomes of the so far evaluated therapies. To enable relevant and translational research, we focused on targetable membrane proteins with high homology degree between species and their presence in five well-characterized solid cancer types, all considered exceptional models of the respective human disease. Table 4 presents a summary of the reviewed targets, their homology between human and dog or cat, the targeted therapies, and the stage of testing (i.e. cell lines, xenografts in mice, or companion animals). While multi-TKIs are more commonly evaluated in veterinary patients, the targeting of individual proteins is mainly restricted to preclinical research. So far, only the targeting of EGFR and FR $\alpha$ has been assessed in the clinic, to treat dogs with invasive TCC. A schematic representation of the most promising and advanced targets in the five different cancer types is depicted in Figure 2.

As evidenced by this review, EGFR-targeting receives large attention not only in human oncology, but also in veterinary research. Canine and feline EGFR share high homology with the human receptor (92\%), making it an interesting target for translational research. In many human cancers, EGFR has proven an invaluable therapeutic target [28]. Multiple EGFR-targeting monoclonal antibodies (e.g. cetuximab and panitumumab) and small molecule inhibitors (e.g. gefitinib and erlotinib) have been approved for the treatment of different human cancers, including HNSCC, colorectal and lung cancer $[28,43]$. In this regard, agents targeting EGFR have also been extensively investigated in canine and feline cell lines of OSCC, OSA, TCC and mammary carcinoma, but only the use of EGF-anthrax toxin has been reported in the clinical setting, for the treatment of canine invasive TCC. This illustrates the large gap between the human and veterinary clinic and points at EGFR targeting as an area where closer collaboration

The existing distance between human and veterinary oncology is also evident in the treatment of mammary carcinoma. A plethora of anti-HER2 agents are used or are under investigation to treat this human neoplasm; on the contrary, published related research remains at the in vitro phase for canine and feline mammary carcinoma. On a positive note, this opens up avenues for the translation of currently successful and HER2-targeting agents from the human to the veterinary clinic, or even of more affordable generics when available. It is striking to see the lack of research regarding targeting of PDGFRs in canine melanoma, HER2 in canine invasive TCC, CXCR4 in mammary carcinoma and IGF-1R in canine osteosarcoma, all highly expressed molecular targets whose targeting is being evaluated in the human clinic for the respective malignancies. The results of the ongoing human trials, when encouraging, will hopefully spur research in the veterinary setting. In the opposite direction, from the veterinary to the human setting, while targeting of folate 
receptors has shown great promise in the treatment of dogs with invasive TCC, no reports have been found in human TCC patients and all seems to indicate that also these patients could benefit from the approach.

Table 4 Overview of the molecular targets and targeted therapy drugs for canine and feline cancers discussed in this review.

\begin{tabular}{|c|c|c|c|c|c|c|}
\hline $\begin{array}{l}\text { Molecular } \\
\text { target }\end{array}$ & $\begin{array}{l}\text { Homology } \\
\operatorname{dog}(\%)^{*}\end{array}$ & $\begin{array}{l}\text { Homology } \\
\text { cat }(\%) *\end{array}$ & Cancer & Targeted agent & Tested in & Ref. \\
\hline \multirow[t]{12}{*}{ EGFR } & 91.5 & 91.7 & HNSCC & Gefitinib & Feline cells & {$[44,45]$} \\
\hline & & & & siRNA & Feline cells & [44] \\
\hline & & & TCC & EGF-anthrax & Dogs & [49] \\
\hline & & & & $\begin{array}{l}\text { toxin } \\
\text { Can225IgG- } \\
\text { IRDye700DX }\end{array}$ & $\begin{array}{l}\text { Canine xenograft } \\
\text { mouse }\end{array}$ & {$[50]$} \\
\hline & & & OSA & Gefitinib & Canine cells & {$[54]$} \\
\hline & & & & Erlotinib & Canine cells & [55] \\
\hline & & & & Erbstatin & Canine cells & {$[56]$} \\
\hline & & & $\mathrm{MC}$ & Cetuximab & Canine cells & {$[61]$} \\
\hline & & & & Gefitinib & Canine/feline cells & {$[62]$} \\
\hline & & & & Can225IgG & Canine cells & {$[63]$} \\
\hline & & & & siRNA & Canine cells & [62] \\
\hline & & & MM & - & - & [67] \\
\hline \multirow[t]{6}{*}{ HER2 } & 93.7 & 92.9 & TCC & - & - & {$[70-72]$} \\
\hline & & & OSA & - & - & {$[77]$} \\
\hline & & & $\mathrm{MC}$ & Trastuzumab & Canine cells & [61] \\
\hline & & & & AG825 & Canine/feline cells & [62] \\
\hline & & & & siRNA & Canine/feline cells & {$[62]$} \\
\hline & & & $\mathrm{MM}$ & - & - & [67] \\
\hline \multirow[t]{2}{*}{ PDGFR $\alpha / \beta$} & $95.9 / 90.2$ & $95.4 / 89.8$ & OSA & - & - & [91] \\
\hline & & & MM & - & - & [95] \\
\hline \multirow[t]{3}{*}{ c-Met } & 89.9 & 90.0 & OSA & shRNA & Canine cells & [98] \\
\hline & & & & РНA-665752 & Canine cells & [98] \\
\hline & & & & PF2362376 & Canine cells & [101] \\
\hline \multirow[t]{2}{*}{ IGF-1R } & 98.2 & 98.1 & OSA & - & - & {$[103,104]$} \\
\hline & & & $\mathrm{MM}$ & - & - & [109-111] \\
\hline IGF-2R & 85.6 & 85.7 & OSA & - & - & [116] \\
\hline \multirow[t]{2}{*}{$\mathrm{FR} \alpha$} & 80.5 & 75.7 & TCC & EC0905 & Dogs & {$[118]$} \\
\hline & & & & EC0531 & Dogs & [119] \\
\hline EP2 & 89.0 & 92.1 & TCC & - & - & [72] \\
\hline MCT1/4 & $88.6 / 87.6$ & $88.4 / 88.0$ & HNSCC & MD-1 & $\begin{array}{l}\text { Feline xenograft } \\
\text { mouse }\end{array}$ & [124] \\
\hline CD146 & 81.9 & 83.6 & MM & - & - & {$[127,128]$} \\
\hline CXCR4 & 95.8 & 94.9 & $\mathrm{MC}$ & - & - & {$[130-132]$} \\
\hline
\end{tabular}

*The protein sequences of the human, canine and feline molecular targets were found in the Protein NCBI database and aligned with Protein BLAST having human as the reference sequence. EGFR: epidermal growth factor receptor; HER2: human epidermal growth factor receptor 2; PDGFR- $\alpha / \beta$ : platelet derived growth factor receptor $\alpha / \beta ;$ c-Met: tyrosine-protein kinase Met; IGF1/2R: insulin-like growth factor $1 / 2$ receptor; FR $\alpha$ : folate receptor $\alpha$; EP2: prostaglandin E2 receptor 2; MCT1/4: monocarboxylate transporter 1/4; CD146: cluster of differentiation 146; CXCR4: C-X-C chemokine receptor type 4; HNSCC: head and neck squamous cell carcinoma; TCC: transitional cell carcinoma; OSA: osteosarcoma; MC: mammary carcinoma; MM: malignant melanoma. 


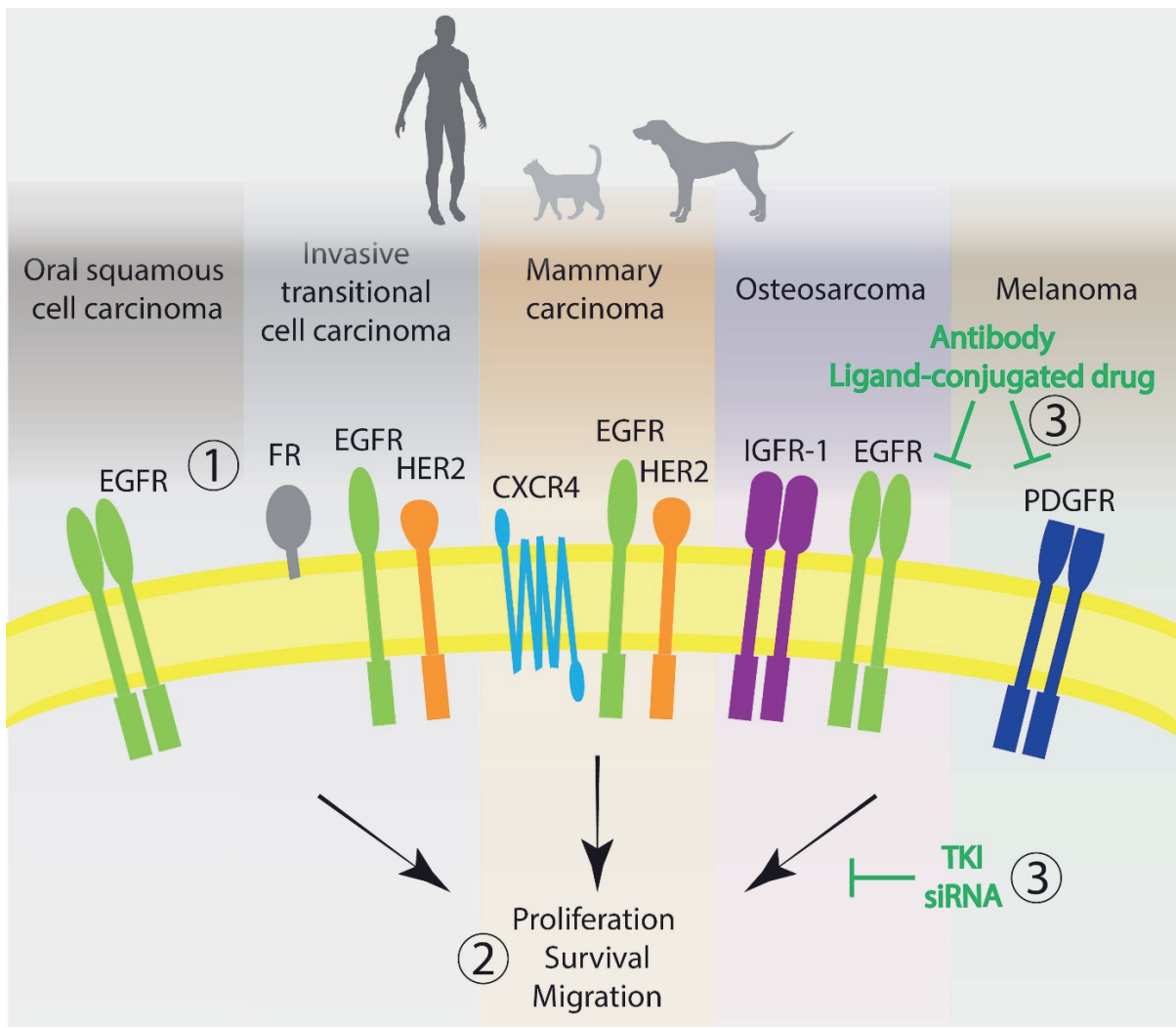

Figure 2 Promising molecular targets for the development of anticancer targeted therapies in humans and companion animals. 1. Most advanced and utilized targets with high homology across species for the five most well-characterized cancer types: EGFR and HER2. Other very promising targets: FR, CXCR4, IGFR-1 and PDGFR. 2. Signal deregulation of these membrane proteins, mostly tyrosine kinase receptors, leads to cell proliferation, survival, migration and tumorigenesis. 3. Categories of therapies most often used to interfere with the targets extracellularly or intracellularly.

EGFR: epidermal growth factor receptor; HER2: human epidermal growth factor receptor 2; PDGFR: platelet derived growth factor receptor; IGF-1R: insulin-like growth factor 1 receptor; FR: folate receptor; CXCR4: C-X-C chemokine receptor type 4. TKI: tyrosine kinase inhibitor.

It is well known that tumor heterogeneity plays a crucial role in the development of drug resistance and patients can clearly benefit from multimodal approaches affecting multiple protumoral pathways. Drawing conclusions from the reviewed data in humans and companion animals, combinations should eventually be considered for the treatment of OSA (including EGFR or PDGFR targeting), HNSCC (with c-Kit inhibition), and mammary carcinoma (using multi-TKIs). As evidenced by this review, the majority of targeted therapies investigated in the veterinary clinic consist of monoclonal antibodies and TKIs. Nowadays, an increasing number of other targeting agents (such as inhibitors of the proteasome and heat shock proteins) are also being assessed, mainly in dogs with hematologic cancers [173]. Advances in the human oncology field and the growing interest in comparative oncology will certainly expand the exploration of more and novel targeted therapies in veterinary patients. 
The benefits that can arise from an intricate relationship between human and veterinary clinic are nicely exemplified by the successful story of the drug toceranib phosphate, the first agent approved for anticancer targeted therapy in dogs. The evaluation of toceranib phosphate in dogs with different spontaneous cancers showed the first evidence for the use of an oral TKI as cancer treatment. These findings helped the development of its structural analogue, sunitinib, which is currently approved as a treatment for several human cancers [9]. Interestingly, the analogue intended for human use received approval in 2006, 3 years before toceranib phosphate was approved in the veterinary clinic, which evidences again the reasonably greater interest in human medicine. Overall, this is an example of successful implementation of comparative oncology in the field of targeted therapy, beneficial for both human and veterinary patients. Within this field, other multi-TKI are also starting to show this benefit across species. Dasatinib, already approved in the human clinic, is now being evaluated in veterinary patients [137]. In the opposite direction, masitinib is currently being evaluated in humans, but was first approved to treat companion animals [138]. Another good example of this comparative approach is the case of NHS-IL12, an antibody-cytokine conjugate. This immunotherapy was first evaluated in dogs with melanoma to study its safety, antitumor activity and pharmacological properties, which informed the design of the first-in-human clinical trials [175].

The limited development of targeted therapies in veterinary oncology can be ascribed to several factors: from biological concerns such as the lack of epitope homology between the human and canine/feline target or the hypersensitive reaction to species antibodies, to economic reasons such as a less attractive veterinary oncology market for the industry or the few opportunities for funding [176]. Clinical trials in companion animals allow for data to be collected relatively fast in comparison to human studies. Still, completion of a study may take over a year and carries associated costs, particularly regarding large scale drug production and the clinical services, facilities and instrumentation. Another difficulty is the recruitment of cases for a veterinary trial, which will be dependent on the incidence of the particular cancer and the relatively early diagnosis to allow for treatment benefit. In this respect, a close collaboration with regional veterinary clinics can definitely be of value. One should not forget that treatment-related toxicities are less acceptable in veterinary medicine, a reason why many successful medicines in the human clinic are not being evaluated in veterinary patients. Opportunities may arise here for targeted therapies, which are known to be tumorselective to some extent, and thus provide a safer setting. To minimize adverse effects, not only is important to verify the presence of the target at the tumor, but also overall target expression and the interactions of the drug with the species target. Although not common, surface expression pattern between species can differ significantly. This led, for instance, to the TGN1412 catastrophe in 2006 [177]. In this respect, theranostic strategies that combine targeted detection before therapy seem a safe approach. Also important is the realization that similar expression pattern does not necessarily translate to identical biological behaviour across species. While most studies in the field rely on immunohistochemistry and PCR techniques to assess expression of a handful of targets, only recently more sophisticated techniques are starting to be introduced that allow for unbiased cross-species analysis, such as wide-genome characterization of the cancer [178] and RNA-sequencing of subpopulations of stroma and neoplastic cells $[14,179]$. Such unbiased analysis enables to investigate similarities between species and to identify new therapeutic targets.

Altogether, when relevant and feasible, breaking the current linearity of drug development programs (mainly from rodents to humans) by incorporating veterinary 
patients can guide the successful implementation of therapeutics in humans, or aid in identifying failure earlier.

\section{Conclusion}

The use of comparative oncology for translational research of targeted therapies is of benefit for both human and veterinary patients. While improving the translational success rate of these therapies from preclinical to clinical studies, dogs and cats get access to otherwise inaccessible novel therapies. Equally important, targeting therapeutic agents approved for human use can be a potential treatment for dogs and cats with tumors expressing the molecular target in question. Clearly, researchers in the field of veterinary and human oncology could help each other and, more importantly, their patients by working closely together.

\section{References}

1. Bray, F.; Ferlay, J.; Soerjomataram, I.; Siegel, R.L.; Torre, L.A.; Jemal, A. Global cancer statistics 2018: GLOBOCAN estimates of incidence and mortality worldwide for 36 cancers in 185 countries. CA. Cancer J. Clin. 2018, 68, 394-424.

2. Miller, K.D.; Nogueira, L.; Mariotto, A.B.; Rowland, J.H.; Yabroff, K.R.; Alfano, C.M.; Jemal, A.; Kramer, J.L.; Siegel, R.L. Cancer treatment and survivorship statistics, 2019. CA. Cancer J. Clin. 2019, 69, 363-385.

3. Wolchok, J. Putting the Immunologic Brakes on Cancer. Cell 2018, 175, 1452-1454.

4. Lee, Y.T.; Tan, Y.J.; Oon, C.E. Molecular targeted therapy: Treating cancer with specificity. Eur. J. Pharmacol. 2018, 834, 188-196.

5. Heem Wong, C.; Wei Siah, K.; Lo, A.W. Estimation of clinical trial success rates and related parameters. Biostatistics 2019, 20, 273-286.

6. Mak, I.W.Y.; Evaniew, N.; Ghert, M. Lost in translation: animal models and clinical trials in cancer treatment. Am. J. Transl. Res. 2014, 6, 114-118.

7. Garden, O.A.; Volk, S.W.; Mason, N.J.; Perry, J.A. Companion animals in comparative oncology : One Medicine in action. Vet. J. 2018, 240, 6-13.

8. Riccardo, F.; Aurisicchio, L.; Impellizeri, J.A.; Cavallo, F. The importance of comparative oncology in translational medicine. Cancer Immunol. Immunother. 2015, 64, 137-148.

9. Regan, D.; Garcia, K.; Thamm, D. Clinical, pathological, and ethical considerations for the conduct of clinical trials in dogs with naturally occurring cancer: a comparative approach to accelerate translational drug development. ILAR J. 2018, 59, 99-110.

10. Cannon, C.M. Cats, Cancer and Comparative Oncology. Vet. Sci. 2015, 2, 111-126.

11. Lindblad-Toh, K.; Wade, C.M.; Mikkelsen, T.S.; Karlsson, E.K.; Jaffe, D.B.; Kamal, M.; Clamp, M.; Chang, J.L.; Kulbokas, E.J.; Zody, M.C.; et al. Genome sequence, comparative analysis and haplotype structure of the domestic dog. Nature 2005, 438, 803-819.

12. Montague, M.J.; Li, G.; Gandolfi, B.; Khan, R.; Aken, B.L.; Searle, S.M.J.; Minx, P.; Hillier, L.W.; Koboldt, D.C.; Davis, B.W.; et al. Comparative analysis of the domestic cat genome reveals genetic signatures underlying feline biology and domestication. Proc. Natl. Acad. Sci. 2014, 111, 17230 LP - 17235.

13. Supsavhad, W.; Dirksen, W.P.; Martin, C.K.; Rosol, T.J. Animal models of head and neck squamous cell carcinoma. Vet. J. 2016, 210, 7-16.

14. Guscetti, F.; Nassiri, S.; Beebe, E.; Brandao, I.R.; Graf, R.; Markkanen, E. Molecular homology between canine spontaneous oral squamous cell carcinomas and human head-and- neck squamous cell carcinomas reveals disease drivers and therapeutic vulnerabilities. Neoplasia 2020, 22, 778788 . 
15. Wypij, J.M. A naturally occurring feline model of head and neck squamous cell carcinoma. Patholog. Res. Int. 2013, 2013, 502197.

16. Argyle, D.J. The Webinar Vet Available online: https://www.thewebinarvet.com/pages/cancershead-neck/.

17. Knapp, D.W.; Dhawan, D.; Ramos-Vara, J.A.; Ratliff, T.L.; Cresswell, G.M.; Utturkar, S.; Sommer, B.C.; Fulkerson, C.M.; Hahn, N.M. Naturally-Occurring Invasive Urothelial Carcinoma in Dogs, a Unique Model to Drive Advances in Managing Muscle Invasive Bladder Cancer in Humans. Front. Oncol. 2020, 9, 1493.

18. Gray, M.; Meehan, J.; Martínez-Pérez, C.; Kay, C.; Turnbull, A.K.; Morrison, L.R.; Pang, L.Y.; Argyle, D. Naturally-Occurring Canine Mammary Tumors as a Translational Model for Human Breast Cancer. Front. Oncol. 2020, 10, 1-17.

19. Hassan, B.B.; Elshafae, S.M.; Supsavhad, W.; Simmons, J.K.; Dirksen, W.P.; Sokkar, S.M.; Rosol, T.J. Feline mammary cancer: novel nude mouse model and molecular characterization of invasion and metastasis genes. Vet. Pathol. 2016, 54, 32-43.

20. Mirabello, L.; Troisi, R.J.; Savage, S.A. International osteosarcoma incidence patterns in children and adolescents, middle ages, and elderly persons. Int. J. Cancer 2009, 125, 229-234.

21. Simpson, S.; Dunning, M.D.; de Brot, S.; Grau-Roma, L.; Mongan, N.P.; Rutland, C.S. Comparative review of human and canine osteosarcoma: morphology, epidemiology, prognosis, treatment and genetics. Acta Vet. Scand. 2017, 59.

22. Gustafson, D.L.; Duval, D.L.; Regan, D.P.; Thamm, D.H. Canine sarcomas as a surrogate for the human disease. Pharmacol Ther 2018, 188, 80-96.

23. Skin Cancer Foundation Available online: https://www.skincancer.org/skin-cancerinformation/skin-cancer-facts/.

24. Prouteau, A.; André, C. Canine Melanomas as Models for Human Melanomas: Clinical, Histological, and Genetic Comparison. Genes (Basel). 2019, 10.

25. Gillard, M.; Cadieu, E.; Brito, C. De; Abadie, J.; Vergier, B.; Devauchelle, P.; Degorce, F.; Dréano, S.; Primot, A.; Dorso, L.; et al. Naturally occurring melanomas in dogs as models for non-UV pathways of human melanomas. Pigment Cell Melanoma Res. 2014, 27, 90-102.

26. Pang, L.; Argyle, D. Veterinary oncology: biology, big data and precision medicine. Vet. J. 2016, 213, 38-45.

27. FETCH a Cure Available online: https://fetchacure.org/resource-library/facts

28. Jue Xu, M.; Johnson, D.E.; Grandis, J.R. EGFR-Targeted Therapies in the Post-Genomic Era. Cancer Metastasis Rev. 2017, 36, 463-473.

29. Ernberg, I.T. Oncogenes and tumor growth factors in breast cancer. A minireview. Acta Oncol. (Madr). 1990, 29, 331-334

30. Pietras, K.; Sjöblom, T.; Rubin, K.; Heldin, C.; Östman, A. PDGF receptors as cancer drug targets. Cancer Cell 2003, 3, 439-443.

31. Zhang, Y.; Xia, M.; Jin, K.; Wang, S.; Wei, H.; Fan, C.; Wu, Y.; Li, X.; Li, X.; Li, G.; et al. Function of the c-Met receptor tyrosine kinase in carcinogenesis and associated therapeutic opportunities. Mol. Cancer 2018, 17.

32. Osher, E.; Macaulay, V.M. Therapeutic targeting of the IGF axis. Cells 2019, 8, 895

33. Martin-Kleiner, I.; Gall Troselj, K. Mannose-6-phosphate/insulin-like growth factor 2 receptor (M6P/IGF2R) in carcinogenesis. Cancer Lett. 2010, 289, 11-22.

34. Scaranti, M.; Cojocaru, E.; Banerjee, S.; Banerji, U. Exploiting the folate receptor $\alpha$ in oncology. Nat. Rev. Clin. Oncol. 2020.

35. Sun, X.; Li, Q. Prostaglandin EP2 receptor: Novel therapeutic target for human cancers (Review). Int. J. Mol. Med. 2018, 42, 1203-1214.

36. Halestrap, A.P. The SLC16 gene family - Structure, role and regulation in health and disease. Mol. Aspects Med. 2013, 34, 337-349.

37. Lei, X.; Guan, C.-W.; Song, Y.; Wang, H. The multifaceted role of CD146/MCAM in the promotion of melanoma progression. Cancer Cell Int. 2015, 15, 3.

38. Zhou, W.; Guo, S.; Liu, M.; Burow, M.E.; Wang, G. Targeting CXCL12/CXCR4 axis in tumor 
immunotherapy. Curr. Med. Chem. 2019, 26, 3026-3041.

39. Looper, J.S.; Malarkey, D.E.; Ruslander, D.; Proulx, D.; Thrall, D.E. Epidermal growth factor receptor expression in feline oral squamous cell carcinomas. Vet. Comp. Oncol. 2006, 4, 33-41.

40. Bergkvist, G.T.; Argyle, D.J.; Morrison, L.; MacIntyre, N.; Hayes, A.; Yool, D.A. Expression of epidermal growth factor receptor (EGFR) and Ki67 in feline oral squamous cell carcinomas (FOSCC). Vet. Comp. Oncol. 2011, 9, 106-117.

41. Bossi, P.; Resteghini, C.; Paielli, N.; Licitra, L.; Pilotti, S.; Perrone, F. Prognostic and predictive value of EGFR in head and neck squamous cell carcinoma. Oncotarget 2016, 7, 74362-74379.

42. Kozakiewicz, P.; Grzybowska-Szatkowska, L. Application of molecular targeted therapies in the treatment of head and neck squamous cell carcinoma. Oncol. Lett. 2018, 15, 7497-7505.

43. Cohen, M.H.; Chen, H.; Shord, S.; Fuchs, C.; He, K.; Zhao, H.; Sickafuse, S.; Keegan, P.; Pazdur, R. Approval summary: Cetuximab in combination with cisplatin or carboplatin and 5-fluorouracil for the first-line treatment of patients with recurrent locoregional or metastatic squamous cell head and neck cancer. Oncologist 2013, 18, 460-466.

44. Bergkvist, G.T.; Argyle, D.J.; Pang, L.Y.; Muirhead, R.; Yool, D.A. Studies on the inhibition of feline EGFR in squamous cell carcinoma: Enhancement of radiosensitivity and rescue of resistance to small molecule inhibitors. Cancer Biol. Ther. 2011, 11, 927-937.

45. Pang, L.Y.; Bergkvist, G.T.; Cervantes-Arias, A.; Yool, D.A.; Muirhead, R.; Argyle, D.J. Identification of tumour initiating cells in feline head and neck squamous cell carcinoma and evidence for gefitinib induced epithelial to mesenchymal transition. Vet. J. 2012, 193, 46-52.

46. Hanazono, K.; Fukumoto, S.; Kawamura, Y.; Endo, Y.; Kadosawa, T.; Iwano, H.; Uchide, T. Epidermal growth factor receptor expression in canine transitional cell carcinoma. J. Vet. Med. Sci. 2015, 77, 1-6.

47. Wright, C.; Mellon, K.; Johnston, P.; Lane, D.P.; Harris, A.L.; Horne, C.H.; Neal, D.E. Expression of mutant p53, c-erbB-2 and the epidermal growth factor receptor in transitional cell carcinoma of the human urinary bladder. Br. J. Cancer 1991, 63, 967-970.

48. Goodspeed, A.; Jean, A.; Theodorescu, D.; Costello, J.C. A gene expression signature predicts bladder cancer cell line sensitivity to EGFR inhibition. Bl. cancer (Amsterdam, Netherlands) 2018, 4, 269-282.

49. Jack, S.; Madhivanan, K.; Ramadesikan, S.; Subramanian, S.; Edwards II, D.F.; Elzey, B.D.; Dhawan, D.; McCluskey, A.; Kischuk, E.M.; Loftis, A.R.; et al. A novel, safe, fast and efficient treatment for Her2-positive and negative bladder cancer utilizing an EGF-anthrax toxin chimera. Int. J. Cancer 2020, 146, 449-460.

50. Nagaya, T.; Okuyama, S.; Ogata, F.; Maruoka, Y.; Knapp, D.W.; Karagiannis, S.N.; FazekasSinger, J.; Choyke, P.L.; LeBlanc, A.K.; Jensen-Jarolim, E.; et al. Near infrared photoimmunotherapy targeting bladder cancer with a canine anti-epidermal growth factor receptor (EGFR) antibody. Oncotarget 2018, 9, 19026-19038

51. Wen, Y.H.; Koeppen, H.; Garcia, R.; Chiriboga, L.; Tarlow, B.D.; Peters, B.A.; Eigenbrot, C.; Yee, H.; Steiner, G.; Greco, M.A. Epidermal growth factor receptor in osteosarcoma: expression and mutational analysis. Hum. Pathol. 2007, 38, 1184-1191.

52. Wang, W.; Zhao, H.; Yao, T.; Gong, H. Advanced development of ErbB family-targeted therapies in osteosarcoma treatment. Invest. New Drugs 2019, 37, 175-183.

53. Selvarajah, G.T.; Verheije, M.H.; Kik, M.; Slob, A.; Rottier, P.J.M.; Mol, J.A.; Kirpensteijn, J. Expression of epidermal growth factor receptor in canine osteosarcoma: Association with clinicopathological parameters and prognosis. Vet. J. 2012, 193, 412-419.

54. McCleese, J.K.; Bear, M.D.; Kulp, S.K.; Mazcko, C.; Khanna, C.; London, C.A. Met interacts with EGFR and Ron in canine osteosarcoma. Vet. Comp. Oncol. 2013, 11, 124-139.

55. Mantovani, F.B.; Morrison, J.A.; Mutsaers, A.J. Effects of epidermal growth factor receptor kinase inhibition on radiation response in canine osteosarcoma cells. BMC Vet. Res. 2016, 12, 82.

56. Mauchle, U.; Selvarajah, G.T.; Mol, J.A.; Kirpensteijn, J.; Verheije, M.H. Identification of antiproliferative kinase inhibitors as potential therapeutic agents to treat canine osteosarcoma. Vet. J. 2015, 205, 281-287.

57. Rimawi, M.F.; Shetty, P.B.; Weiss, H.L.; Schiff, R.; Osborne, C.K.; Chamness, G.C.; Elledge, R.M. Epidermal growth factor receptor expression in breast cancer association with biologic phenotype 
and clinical outcomes. Cancer 2010, 116, 1234-1242.

58. Maennling, A.E.; Tur, M.K.; Niebert, M.; Klockenbring, T.; Zeppernick, F.; Gattenlöhner, S.; Meinhold-Heerlein, I.; Hussain, A.F. Molecular targeting therapy against EGFR family in breast cancer: progress and future potentials. Cancers (Basel). 2019, 11, 1826.

59. Gama, A.; Gärtner, F.; Alves, A.; Schmitt, F. Immunohistochemical expression of Epidermal Growth Factor Receptor (EGFR) in canine mammary tissues. Res. Vet. Sci. 2009, 87, 432-437.

60. Hassan, B.B.; Elshafae, S.M.; Supsavhad, W.; Simmons, J.K.; Dirksen, W.P.; Sokkar, S.M.; Rosol, T.J. Feline mammary cancer. Vet. Pathol. 2017, 54, 32-43.

61. Singer, J.; Weichselbaumer, M.; Stockner, T.; Mechtcheriakova, D.; Sobanov, Y.; Bajna, E.; Wrba, F.; Horvat, R.; Thalhammer, J.G.; Willmann, M.; et al. Comparative oncology: ErbB-1 and ErbB2 homologues in canine cancer are susceptible to cetuximab and trastuzumab targeting. Mol. Immunol. 2012, 50, 200-209.

62. Gray, M.E.; Lee, S.; McDowell, A.L.; Erskine, M.; Loh, Q.T.M.; Grice, O.; Argyle, D.J.; Bergkvist, G.T. Dual targeting of EGFR and ERBB2 pathways produces a synergistic effect on cancer cell proliferation and migration in vitro. Vet. Comp. Oncol. 2017, 15, 890-909.

63. Singer, J.; Fazekas, J.; Wang, W.; Weichselbaumer, M.; Matz, M.; Mader, A.; Steinfellner, W.; Meitz, S.; Mechtcheriakova, D.; Sobanov, Y.; et al. Generation of a canine anti-EGFR (ErbB-1) antibody for passive immunotherapy in dog cancer patients. Mol. Cancer Ther. 2014, 13, 17771790 .

64. Boone, B.; Jacobs, K.; Ferdinande, L.; Taildeman, J.; Lambert, J.; Peeters, M.; Bracke, M.; Pauwels, P.; Brochez, L. EGFR in melanoma: clinical significance and potential therapeutic target. J. Cutan. Pathol. 2011, 38, 492-502.

65. Patel, S.P.; Kim, K.B.; Papadopoulos, N.E.; Hwu, W.-J.; Hwu, P.; Prieto, V.G.; Bar-Eli, M.; Zigler, M.; Dobroff, A.; Bronstein, Y.; et al. A phase II study of gefitinib in patients with metastatic melanoma. Melanoma Res. 2011, 21, 357-363.

66. Mudigonda, T. V; Wyman, K.; Spigel, D.R.; Dahlman, K.B.; Greco, F.A.; Puzanov, I.; Kelley, M.C.; Hainsworth, J.D.; Sosman, J.A.; Johnson, D.B. A phase II trial of erlotinib and bevacizumab for patients with metastatic melanoma. Pigment Cell Melanoma Res. 2016, 29, 101-103.

67. Veloso, E.S.; Gonçalves, I.N.N.; Silveira, T.L.; Oliveira, F.S.; Vieira, D.S.; Cassali, G.D.; Del Puerto, H.L.; Ferreira, E. Diverse roles of epidermal growth factors receptors in oral and cutaneous canine melanomas. BMC Vet. Res. 2020, 16, 24.

68. Rahman, M.M.; Lai, Y.-C.; Husna, A.A.; Chen, H.-W.; Tanaka, Y.; Kawaguchi, H.; Hatai, H.; Miyoshi, N.; Nakagawa, T.; Fukushima, R.; et al. Transcriptome analysis of dog oral melanoma and its oncogenic analogy with human melanoma. Oncol. Rep. 2020, 43, 16-30.

69. Coogan, C.L.; Estrada, C.R.; Kapur, S.; Bloom, K.J. HER-2/neu protein overexpression and gene amplification in human transitional cell carcinoma of the bladder. Urology 2004, 63, 786-790.

70. Millanta, F.; Impellizeri, J.; McSherry, L.; Rocchigiani, G.; Aurisicchio, L.; Lubas, G. Overexpression of HER-2 via immunohistochemistry in canine urinary bladder transitional cell carcinoma - A marker of malignancy and possible therapeutic target. Vet. Comp. Oncol. 2018, 16, 297-300.

71. Tsuboi, M.; Sakai, K.; Maeda, S.; Chambers, J.K.; Yonezawa, T.; Matsuki, N.; Uchida, K.; Nakayama, H. Assessment of HER2 expression in canine urothelial carcinoma of the urinary bladder. Vet. Pathol. 2019, 56, 369-376.

72. Maeda, S.; Tomiyasu, H.; Tsuboi, M.; Inoue, A.; Ishihara, G.; Uchikai, T.; Chambers, J.K.; Uchida, K.; Yonezawa, T.; Matsuki, N. Comprehensive gene expression analysis of canine invasive urothelial bladder carcinoma by RNA-Seq. BMC Cancer 2018, 18, 472.

73. Koshkin, V.S.; O’Donnell, P.; Yu, E.Y.; Grivas, P. Systematic review: Targeting HER2 in bladder cancer. Bl. Cancer 2019, 5, 1-12.

74. Tabak, S.A.; Khalifa, S.E.; Fathy, Y. HER-2 immunohistochemical expression in bone sarcomas: a new hope for osteosarcoma patients. Open access Maced. J. Med. Sci. 2018, 6, 1555-1560.

75. Thomas, D.G.; Giordano, T.J.; Sanders, D.; Biermann, J.S.; Baker, L. Absence of HER2/neu gene expression in osteosarcoma and skeletal Ewings sarcoma. Clin. Cancer Res. 2002, 8, 788-793.

76. Kilpatrick, S.E.; Geisinger, K.R.; King, T.S.; Sciarrotta, J.; Ward, W.G.; Gold, S.H.; Bos, G.D. Clinicopathologic analysis of HER-2/neu immunoexpression among various histologic subtypes 
and grades of osteosarcoma. Mod. Pathol. 2001, 14, 1277-1283.

77. Flint, A.F.; U'Ren, L.; Legare, M.E.; Withrow, S.J.; Dernell, W.; Hanneman, W.H. Overexpression of the erbb-2 proto-oncogene in canine osteosarcoma cell lines and tumors. Vet. Pathol. 2004, 41, 291-296.

78. Ebb, D.; Meyers, P.; Grier, H.; Bernstein, M.; Gorlick, R.; Lipshultz, S.E.; Krailo, M.; Devidas, M.; Barkauskas, D.A.; Siegal, G.P.; et al. Phase II trial of trastuzumab in combination with cytotoxic chemotherapy for treatment of metastatic osteosarcoma with human epidermal growth factor receptor 2 overexpression: a report from the children's oncology group. J. Clin. Oncol. 2012, 30, 2545-2551.

79. Ahmed, N.; Brawley, V.S.; Hegde, M.; Robertson, C.; Ghazi, A.; Gerken, C.; Liu, E.; Dakhova, O.; Ashoori, A.; Corder, A.; et al. Human epidermal growth factor receptor 2 (HER2) -specific chimeric antigen receptor-modified T Cells for the immunotherapy of HER2-positive sarcoma. $J$. Clin. Oncol. 2015, 33, 1688-1696.

80. Mason, N.J.; Gnanandarajah, J.S.; Engiles, J.B.; Gray, F.; Laughlin, D.; Gaurnier-Hausser, A.; Wallecha, A.; Huebner, M.; Paterson, Y. Immunotherapy with a HER2-Targeting Listeria Induces HER2-Specific Immunity and Demonstrates Potential Therapeutic Effects in a Phase I Trial in Canine Osteosarcoma. Clin. Cancer Res. 2016, 22, 4380 LP - 4390.

81. Ménard, S.; Tagliabue, E.; Campiglio, M.; Pupa, S.M. Role of HER2 gene overexpression in breast carcinoma. J. Cell. Physiol. 2000, 182, 150-162.

82. Marques, C.; Correia, J.; Ferreira, F. HER2-positive feline mammary carcinoma. Aging (Albany. NY). 2016, 8, 1574-1575.

83. De Maria, R.; Olivero, M.; Iussich, S.; Nakaichi, M.; Murata, T.; Biolatti, B.; Di Renzo, M.F. Spontaneous feline mammary carcinoma is a model of HER 2 overexpressing poor prognosis human breast cancer. Cancer Res. 2005, 65, 907 LP - 912.

84. Ressel, L.; Puleio, R.; Loria, G.R.; Vannozzi, I.; Millanta, F.; Caracappa, S.; Poli, A. HER-2 expression in canine morphologically normal, hyperplastic and neoplastic mammary tissues and its correlation with the clinical outcome. Res. Vet. Sci. 2013, 94, 299-305.

85. Hsu, W.-L.; Huang, H.-M.; Liao, J.-W.; Wong, M.-L.; Chang, S.-C. Increased survival in dogs with malignant mammary tumours overexpressing HER-2 protein and detection of a silent single nucleotide polymorphism in the canine HER-2 gene. Vet. J. 2009, 180, 116-123.

86. Kluger, H.M.; DiVito, K.; Berger, A.J.; Halaban, R.; Ariyan, S.; Camp, R.L.; Rimm, D.L. Her2/neu is not a commonly expressed therapeutic target in melanoma - a large cohort tissue microarray study. Melanoma Res. 2004, 14.

87. Bodey, B.; Bodey, J.B.; Gröger, A.M.; Luck, J. V; Siegel, S.E.; Taylor, C.R.; Kaiser, H.E. Clinical and prognostic significance of the expression of the c-erbB-2 and c-erbB-3 oncoproteins in primary and metastatic malignant melanomas and breast carcinomas. Anticancer Res. 1997, 17, 1319-1330.

88. Ma, J.; Han, H.; Liu, D.; Li, W.; Feng, H.; Xue, X.; Wu, X.; Niu, G.; Zhang, G.; Zhao, Y.; et al. HER2 as a promising target for cytotoxicity T cells in human melanoma therapy. PLoS One 2013, 8, e73261-e73261.

89. Kubo, T.; Piperdi, S.; Rosenblum, J.; Antonescu, C.R.; Chen, W.; Kim, H.-S.; Huvos, A.G.; Sowers, R.; Meyers, P.A.; Healey, J.H.; et al. Platelet-derived growth factor receptor as a prognostic marker and a therapeutic target for imatinib mesylate therapy in osteosarcoma. Cancer 2008, 112, 2119-2129.

90. Xu, J.; Xie, L.; Guo, W. PDGF/PDGFR effects in osteosarcoma and the "add-on" strategy. Clin. Sarcoma Res. 2018, 8, 15.

91. Maniscalco, L.; Iussich, S.; Morello, E.; Martano, M.; Biolatti, B.; Riondato, F.; Salda, L. Della; Romanucci, M.; Malatesta, D.; Bongiovanni, L.; et al. PDGFs and PDGFRs in canine osteosarcoma: New targets for innovative therapeutic strategies in comparative oncology. Vet. J. 2013, 195, 41-47.

92. D’Arcangelo, D.; Facchiano, F.; Nassa, G.; Stancato, A.; Antonini, A.; Rossi, S.; Senatore, C.; Cordella, M.; Tabolacci, C.; Salvati, A.; et al. PDGFR-alpha inhibits melanoma growth via CXCL10/IP-10: a multi-omics approach. Oncotarget 2016, 7, 77257-77275.

93. Barnhill, R.L.; Xiao, M.; Graves, D.; Antoniades, H.N. Expression of platelet-derived growth factor (PDGF)-A, PDGF-B and the PDGF-alpha receptor, but not the PDGF-beta receptor, in human 
malignant melanoma in vivo. Br. J. Dermatol. 1996, 135, 898-904.

94. Papadopoulos, N.; Lennartsson, J. The PDGF/PDGFR pathway as a drug target. Mol. Aspects Med. 2018, 62, 75-88.

95. Iussich, S.; Maniscalco, L.; Di Sciuva, A.; Iotti, B.; Morello, E.; Martano, M.; Gattino, F.; Buracco, P.; De Maria, R. PDGFRs expression in dogs affected by malignant oral melanomas: correlation with prognosis. Vet. Comp. Oncol. 2017, 15, 462-469.

96. Hassan, S.E.; Bekarev, M.; Kim, M.Y.; Lin, J.; Piperdi, S.; Gorlick, R.; Geller, D.S. Cell surface receptor expression patterns in osteosarcoma. Cancer 2012, 118, 740-749.

97. Scotlandi, K.; Baldini, N.; Oliviero, M.; Di Renzo, M.F.; Martano, M.; Serra, M.; Manara, M.C.; Comoglio, P.M.; Ferracini, R. Expression of Met/hepatocyte growth factor receptor gene and malignant behavior of musculoskeletal tumors. Am. J. Pathol. 1996, 149, 1209-1219.

98. De Maria, R.; Miretti, S.; Iussich, S.; Olivero, M.; Morello, E.; Bertotti, A.; Christensen, J.G.; Biolatti, B.; Levine, R.A.; Buracco, P.; et al. met oncogene activation qualifies spontaneous canine osteosarcoma as a suitable pre-clinical model of human osteosarcoma. J. Pathol. 2009, 218, 399408.

99. Fioramonti, M.; Fausti, V.; Pantano, F.; Iuliani, M.; Ribelli, G.; Lotti, F.; Pignochino, Y.; Grignani, G.; Santini, D.; Tonini, G.; et al. Cabozantinib affects osteosarcoma growth through a direct effect on tumor cells and modifications in bone microenvironment. Sci. Rep. 2018, 8, 4177.

100. Sampson, E.R.; Martin, B.A.; Morris, A.E.; Xie, C.; Schwarz, E.M.; O’Keefe, R.J.; Rosier, R.N. The orally bioavailable met inhibitor PF-2341066 inhibits osteosarcoma growth and osteolysis/matrix production in a xenograft model. J. Bone Miner. Res. 2011, 26, 1283-1294.

101. Liao, A.T.; McCleese, J.; Kamerling, S.; Christensen, J.; London, C.A. A novel small molecule Met inhibitor, PF2362376, exhibits biological activity against osteosarcoma. Vet. Comp. Oncol. 2007, 5, 177-196.

102. Wang, Y.-H.; Han, X.-D.; Qiu, Y.; Xiong, J.; Yu, Y.; Wang, B.; Zhu, Z.-Z.; Qian, B.-P.; Chen, Y.X.; Wang, S.-F.; et al. Increased expression of insulin-like growth factor-1 receptor is correlated with tumor metastasis and prognosis in patients with osteosarcoma. J. Surg. Oncol. 2012, 105, 235243.

103. MacEwen, E.G.; Pastor, J.; Kutzke, J.; Tsan, R.; Kurzman, I.D.; Thamm, D.H.; Wilson, M.; Radinsky, R. IGF-1 receptor contributes to the malignant phenotype in human and canine osteosarcoma. J. Cell. Biochem. 2004, 92, 77-91.

104. Maniscalco, L.; Iussich, S.; Morello, E.; Martano, M.; Gattino, F.; Miretti, S.; Biolatti, B.; Accornero, P.; Martignani, E.; Sánchez-Céspedes, R.; et al. Increased expression of insulin-like growth factor-1 receptor is correlated with worse survival in canine appendicular osteosarcoma. Vet. J. 2015, 205, 272-280.

105. Pappo, A.S.; Vassal, G.; Crowley, J.J.; Bolejack, V.; Hogendoorn, P.C.W.; Chugh, R.; Ladanyi, M.; Grippo, J.F.; Dall, G.; Staddon, A.P.; et al. A phase 2 trial of R1507, a monoclonal antibody to the insulin-like growth factor-1 receptor (IGF-1R), in patients with recurrent or refractory rhabdomyosarcoma, osteosarcoma, synovial sarcoma, and other soft tissue sarcomas: results of a Sarcoma Alliance. Cancer 2014, 120, 2448-2456.

106. Li, Y.; Liu, Q.; He, H.; Luo, W. The possible role of insulin-like growth factor-1 in osteosarcoma. Curr. Probl. Cancer 2019, 43, 228-235.

107. Yerushalmi, R.; Gelmon, K.A.; Leung, S.; Gao, D.; Cheang, M.; Pollak, M.; Turashvili, G.; Gilks, B.C.; Kennecke, H. Insulin-like growth factor receptor (IGF-1R) in breast cancer subtypes. Breast Cancer Res. Treat. 2012, 132, 131-142.

108. Ekyalongo, R.C.; Yee, D. Revisiting the IGF-1R as a breast cancer target. NPJ Precis. Oncol. 2017, 1,14 .

109. Jaillardon, L.; Abadie, J.; Godard, T.; Campone, M.; Loussouarn, D.; Siliart, B.; Nguyen, F. The $\mathrm{dog}$ as a naturally-occurring model for insulin-like growth factor type 1 receptor-overexpressing breast cancer: an observational cohort study. BMC Cancer 2015, 15, 664.

110. Dolka, I.; Motyl, T.; Malicka, E.; Sapierzyński, R.; Fabisiak, M. Relationship between receptors for insulin-like growth factor-I, steroid hormones and apoptosis-associated proteins in canine mammary tumors. Pol. J. Vet. Sci. 2011, 14, 245-251.

111. Klopfleisch, R.; Hvid, H.; Klose, P.; Da Costa, A.; Gruber, A.D. Insulin receptor is expressed in 
normal canine mammary gland and benign adenomas but decreased in metastatic canine mammary carcinomas similar to human breast cancer. Vet. Comp. Oncol. 2010, 8, 293-301.

112. Kanter-Lewensohn, L.; Dricu, A.; Girnita, L.; Wejde, J.; Larsson, O. Expression of insulin-like growth factor-1 receptor (IGF-1R) and $\mathrm{p} 27 \mathrm{Kip} 1$ in melanocyte tumors: a potential regulatory role of IGF-1 pathway in distribution of p27Kip1 between different cyclins. Growth Factors 2000, 17, 193-202.

113. Capoluongo, E. Insulin-like growth factor system and sporadic malignant melanoma. Am. J. Pathol. 2011, 178, 26-31.

114. Thamm, D.H.; Huelsmeyer, M.K.; Mitzey, A.M.; Qurollo, B.; Rose, B.J.; Kurzman, I.D. RT-PCRbased tyrosine kinase display profiling of canine melanoma: IGF-1 receptor as a potential therapeutic target. Melanoma Res. 2010, 20.

115. Simpson, A.; Petnga, W.; Macaulay, V.M.; Weyer-Czernilofsky, U.; Bogenrieder, T. Insulin-like growth factor (IGF) pathway targeting in cancer: Role of the IGF axis and opportunities for future combination studies. Target. Oncol. 2017, 12, 571-597.

116. Karkare, S.; Allen, K.J.H.; Jiao, R.; Malo, M.E.; Dawicki, W.; Helal, M.; Godson, D.L.; Dickinson, R.; MacDonald-Dickinson, V.; Yang, R.; et al. Detection and targeting insulin growth factor receptor type 2 (IGF2R) in osteosarcoma PDX in mouse models and in canine osteosarcoma tumors. Sci. Rep. 2019, 9, 11476.

117. Geller, D.S.; Morris, J.; Revskaya, E.; Kahn, M.; Zhang, W.; Piperdi, S.; Park, A.; Koirala, P.; Guzik, H.; Hall, C.; et al. Targeted therapy of osteosarcoma with radiolabeled monoclonal antibody to an insulin-like growth factor-2 receptor (IGF2R). Nucl. Med. Biol. 2016, 43, 812-817.

118. Dhawan, D.; Ramos-Vara, J.A.; Naughton, J.F.; Cheng, L.; Low, P.S.; Rothenbuhler, R.; Leamon, C.P.; Parker, N.; Klein, P.J.; Vlahov, I.R.; et al. Targeting folate receptors to treat invasive urinary bladder cancer. Cancer Res. 2013, 73, 875 LP - 884.

119. Szigetvari, N.M.; Dhawan, D.; Ramos-Vara, J.A.; Leamon, C.P.; Klein, P.J.; Ruple, A.A.; Heng, H.G.; Pugh, M.R.; Rao, S.; Vlahov, I.R.; et al. Phase I/II clinical trial of the targeted chemotherapeutic drug, folate-tubulysin, in dogs with naturally-occurring invasive urothelial carcinoma. Oncotarget 2018, 9, 37042-37053.

120. Kashiwagi, E.; Inoue, S.; Mizushima, T.; Chen, J.; Ide, H.; Kawahara, T.; Reis, L.O.; Baras, A.S.; Netto, G.J.; Miyamoto, H. Prostaglandin receptors induce urothelial tumourigenesis as well as bladder cancer progression and cisplatin resistance presumably via modulating PTEN expression. Br. J. Cancer 2018, 118, 213-223.

121. von der Emde, L.; Goltz, D.; Latz, S.; Müller, S.C.; Kristiansen, G.; Ellinger, J.; Syring, I. Prostaglandin receptors EP1-4 as a potential marker for clinical outcome in urothelial bladder cancer. Am. J. Cancer Res. 2014, 4, 952-962.

122. Simões-Sousa, S.; Granja, S.; Pinheiro, C.; Fernandes, D.; Longatto-Filho, A.; Laus, A.C.; Alves, C.D.C.; Suárez-Peñaranda, J.M.; Pérez-Sayáns, M.; Lopes Carvalho, A.; et al. Prognostic significance of monocarboxylate transporter expression in oral cavity tumors. Cell Cycle 2016, 15, 1865-1873.

123. Curry, J.M.; Tuluc, M.; Whitaker-Menezes, D.; Ames, J.A.; Anantharaman, A.; Butera, A.; Leiby, B.; Cognetti, D.M.; Sotgia, F.; Lisanti, M.P.; et al. Cancer metabolism, stemness and tumor recurrence: MCT1 and MCT4 are functional biomarkers of metabolic symbiosis in head and neck cancer. Cell Cycle 2013, 12, 1371-1384.

124. Khammanivong, A.; Saha, J.; Spartz, A.K.; Sorenson, B.S.; Bush, A.G.; Korpela, D.M.; Gopalakrishnan, R.; Jonnalagadda, S.; Mereddy, V.R.; O’Brien, T.D.; et al. A novel MCT1 and MCT4 dual inhibitor reduces mitochondrial metabolism and inhibits tumour growth of feline oral squamous cell carcinoma. Vet. Comp. Oncol. 2019, 18.

125. Pearl, R.A.; Pacifico, M.D.; Richman, P.I.; Wilson, G.D.; Grover, R. Stratification of patients by melanoma cell adhesion molecule (MCAM) expression on the basis of risk: implications for sentinel lymph node biopsy. J. Plast. Reconstr. Aesthetic Surg. 2008, 61, 265-271.

126. Wei, W.; Jiang, D.; Ehlerding, E.B.; Barnhart, T.E.; Yang, Y.; Engle, J.W.; Luo, Q.-Y.; Huang, P.; Cai, W. CD146-targeted multimodal image-guided photoimmunotherapy of melanoma. Adv. Sci. 2019, 6, 1801237.

127. Abou Asa, S. Immunohistochemical expression of MCAM/CD146 in canine melanoma. J. Comp. Pathol. 2017, 157, 27-33. 
128. Schmid, F.; Brodesser, D.; Reifinger, M.; Forte, S.; Semp, P.; Eberspächer-Schweda, M.C.; Wolschek, M.; Brandt, S.; Kleiter, M.; Pratscher, B. Canine oral primary melanoma cells exhibit shift to mesenchymal phenotype and phagocytic behaviour. Vet. Comp. Oncol. 2019, 17, 211-220.

129. Pernas, S.; Martin, M.; Kaufman, P.A.; Gil-Martin, M.; Gomez Pardo, P.; Lopez-Tarruella, S.; Manso, L.; Ciruelos, E.; Perez-Fidalgo, J.A.; Hernando, C.; et al. Balixafortide plus eribulin in HER2-negative metastatic breast cancer: a phase 1, single-arm, dose-escalation trial. Lancet Oncol. 2018, 19, 812-824.

130. Marques, C.S.; Santos, A.R.; Gameiro, A.; Correia, J.; Ferreira, F. CXCR4 and its ligand CXCL12 display opposite expression profiles in feline mammary metastatic disease, with the exception of HER2-overexpressing tumors. BMC Cancer 2018, 18, 741 .

131. Ferrari, A.; Petterino, C.; Ratto, A.; Campanella, C.; Wurth, R.; Thellung, S.; Vito, G.; Barbieri, F.; Florio, T. CXCR4 expression in feline mammary carcinoma cells: evidence of a proliferative role for the SDF-1/CXCR4 axis. BMC Vet. Res. 2012, 8, 27.

132. Hussain, S.; Saxena, S.; Shrivastava, S.; Mohanty, A.K.; Kumar, S.; Singh, R.J.; Kumar, A.; Wani, S.A.; Gandham, R.K.; Kumar, N.; et al. Gene expression profiling of spontaneously occurring canine mammary tumours: Insight into gene networks and pathways linked to cancer pathogenesis. PLoS One 2018, 13, e0208656-e0208656.

133. Gschwind, A.; Fischer, O.M.; Ullrich, A. The discovery of receptor tyrosine kinases: targets for cancer therapy. Nat. Rev. Cancer 2004, 4, 361-370.

134. Wiles, V.; Hohenhaus, A.; Lamb, K.; Zaidi, B.; Camps-Palau, M.; Leibman, N. Retrospective evaluation of toceranib phosphate (Palladia) in cats with oral squamous cell carcinoma. J. Feline Med. Surg. 2016, 19, 185-193.

135. London, C.; Mathie, T.; Stingle, N.; Clifford, C.; Haney, S.; Klein, M.K.; Beaver, L.; Vickery, K.; Vail, D.M.; Hershey, B.; et al. Preliminary evidence for biologic activity of toceranib phosphate (Palladia(®)) in solid tumours. Vet. Comp. Oncol. 2012, 10, 194-205.

136. Papaetis, G.S.; Syrigos, K.N. Sunitinib. BioDrugs 2009, 23, 377-389.

137. Montero, J.C.; Seoane, S.; Ocaña, A.; Pandiella, A. Inhibition of Src family kinases and receptor tyrosine kinases by dasatinib: possible combinations in solid tumors. Clin. Cancer Res. 2011, 17, 5546 LP - 5552.

138. Dubreuil, P.; Letard, S.; Ciufolini, M.; Gros, L.; Humbert, M.; Castéran, N.; Borge, L.; Hajem, B.; Lermet, A.; Sippl, W.; et al. Masitinib (AB1010), a potent and selective tyrosine kinase inhibitor targeting KIT. PLoS One 2009, 4, e7258-e7258.

139. Rathore, K.; Alexander, M.; Cekanova, M. Piroxicam inhibits Masitinib-induced cyclooxygenase 2 expression in oral squamous cell carcinoma cells in vitro. Transl. Res. 2014, 164, 158-168.

140. Gustafson, T.L.; Biller, B. Use of toceranib phosphate in the treatment of canine bladder tumors: 37 cases. J. Am. Anim. Hosp. Assoc. 2019, 55, 243-248.

141. Davis, L.E.; Hofmann, N.E.; Li, G.; Huang, E.T.; Loriaux, M.M.; Bracha, S.; Helfand, S.C.; Mata, J.E.; Marley, K.; Mansoor, A.; et al. A case study of personalized therapy for osteosarcoma. Pediatr. Blood Cancer 2013, 60, 1313-1319.

142. Marley, K.; Gullaba, J.; Seguin, B.; Gelberg, H.B.; Helfand, S.C. Dasatinib modulates invasive and migratory properties of canine osteosarcoma and has therapeutic potential in affected dogs. Transl. Oncol. 2015, 8, 231-238.

143. Rossi, F.; Sabattini, S.; Vascellari, M.; Marconato, L. The impact of toceranib, piroxicam and thalidomide with or without hypofractionated radiation therapy on clinical outcome in dogs with inflammatory mammary carcinoma. Vet. Comp. Oncol. 2018, 16, 497-504.

144. London, C.A.; Hannah, A.L.; Zadovoskaya, R.; Chien, M.B.; Kollias-Baker, C.; Rosenberg, M.; Downing, S.; Post, G.; Boucher, J.; Shenoy, N.; et al. Phase I dose-escalating study of SU11654, a small molecule receptor tyrosine kinase inhibitor, in dogs with spontaneous malignancies. Clin. Cancer Res. 2003, 9, 2755-2768.

145. Wouda, R.M.; Hocker, S.E.; Higginbotham, M.L. Safety evaluation of combination carboplatin and toceranib phosphate (Palladia) in tumour-bearing dogs: A phase I dose finding study. Vet. Comp. Oncol. 2018, 16, E52-E60.

146. Choong, N.W.; Kozloff, M.; Taber, D.; Hu, H.S.; Wade, J.; Ivy, P.; Karrison, T.G.; Dekker, A.; Vokes, E.E.; Cohen, E.E.W. Phase II study of sunitinib malate in head and neck squamous cell 
carcinoma. Invest. New Drugs 2010, 28, 677-683.

147. Machiels, J.-P.H.; Henry, S.; Zanetta, S.; Kaminsky, M.-C.; Michoux, N.; Rommel, D.; Schmitz, S.; Bompas, E.; Dillies, A.-F.; Faivre, S.; et al. Phase II study of sunitinib in recurrent or metastatic squamous cell carcinoma of the head and neck: GORTEC 2006-01. J. Clin. Oncol. 2009, 28, 2128.

148. Zehnder, A.; Graham, J.; Antonissen, G. Update on cancer treatment in exotics. Vet. Clin. North Am. Exot. Anim. Pract. 2018, 21, 465-509.

149. Ongkeko, W.M.; Altuna, X.; Weisman, R.A.; Wang-Rodriguez, J. Expression of protein tyrosine kinases in head and neck squamous cell carcinomas. Am. J. Clin. Pathol. 2005, 124, 71-76.

150. Zahoor, H.; Mir, M.C.; Barata, P.C.; Stephenson, A.J.; Campbell, S.C.; Fergany, A.; Dreicer, R.; Garcia, J.A. Phase II trial of continuous treatment with sunitinib in patients with high-risk (BCGrefractory) non-muscle invasive bladder cancer. Invest. New Drugs 2019, 37, 1231-1238.

151. Grivas, P.D.; Daignault, S.; Tagawa, S.T.; Nanus, D.M.; Stadler, W.M.; Dreicer, R.; Kohli, M.; Petrylak, D.P.; Vaughn, D.J.; Bylow, K.A.; et al. Double-blind, randomized, phase 2 trial of maintenance sunitinib versus placebo after response to chemotherapy in patients with advanced urothelial carcinoma. Cancer 2014, 120, 692-701.

152. Bellmunt, J.; González-Larriba, J.L.; Prior, C.; Maroto, P.; Carles, J.; Castellano, D.; Mellado, B.; Gallardo, E.; Perez-Gracia, J.L.; Aguilar, G.; et al. Phase II study of sunitinib as first-line treatment of urothelial cancer patients ineligible to receive cisplatin-based chemotherapy: baseline interleukin- 8 and tumor contrast enhancement as potential predictive factors of activity. Ann. Oncol. 2011, 22, 2646-2653.

153. Walters, L.; Martin, O.; Price, J.; Sula, M.M. Expression of receptor tyrosine kinase targets PDGFR- $\beta$, VEGFR2 and KIT in canine transitional cell carcinoma. Vet. Comp. Oncol. 2018, 16, E117-E122.

154. Kumar, R.M.R.; Arlt, M.J.; Kuzmanov, A.; Born, W.; Fuchs, B. Sunitinib malate (SU-11248) reduces tumour burden and lung metastasis in an intratibial human xenograft osteosarcoma mouse model. Am. J. Cancer Res. 2015, 5, 2156-2168.

155. Gieger, T.L.; Nettifee-Osborne, J.; Hallman, B.; Johannes, C.; Clarke, D.; Nolan, M.W.; Williams, L.E. The impact of carboplatin and toceranib phosphate on serum vascular endothelial growth factor (VEGF) and metalloproteinase-9 (MMP-9) levels and survival in canine osteosarcoma. Can. J. Vet. Res. 2017, 81, 199-205.

156. Kim, C.; Matsuyama, A.; Mutsaers, A.J.; Woods, J.P. Retrospective evaluation of toceranib (Palladia) treatment for canine metastatic appendicular osteosarcoma. Can. Vet. J. $=$ La Rev. Vet. Can. 2017, 58, 1059-1064.

157. Laver, T.; London, C.A.; Vail, D.M.; Biller, B.J.; Coy, J.; Thamm, D.H. Prospective evaluation of toceranib phosphate in metastatic canine osteosarcoma. Vet. Comp. Oncol. 2018, 16, E23-E29.

158. London, C.A.; Gardner, H.L.; Mathie, T.; Stingle, N.; Portela, R.; Pennell, M.L.; Clifford, C.A.; Rosenberg, M.P.; Vail, D.M.; Williams, L.E.; et al. Impact of toceranib/piroxicam/cyclophosphamide maintenance therapy on outcome of dogs with appendicular osteosarcoma following amputation and carboplatin chemotherapy: a multiinstitutional study. PLoS One 2015, 10, e0124889-e0124889.

159. Sánchez-Céspedes, R.; Accornero, P.; Miretti, S.; Martignani, E.; Gattino, F.; Maniscalco, L.; Gola, C.; Iussich, S.; Martano, M.; Morello, E.; et al. In vitro and in vivo effects of toceranib phosphate on canine osteosarcoma cell lines and xenograft orthotopic models. Vet. Comp. Oncol. 2020, 18, $117-127$.

160. Schuetze, S.M.; Wathen, J.K.; Lucas, D.R.; Choy, E.; Samuels, B.L.; Staddon, A.P.; Ganjoo, K.N.; von Mehren, M.; Chow, W.A.; Loeb, D.M.; et al. SARC009: Phase 2 study of dasatinib in patients with previously treated, high-grade, advanced sarcoma. Cancer 2016, 122, 868-874.

161. Crown, J.P.; Diéras, V.; Staroslawska, E.; Yardley, D.A.; Bachelot, T.; Davidson, N.; Wildiers, H.; Fasching, P.A.; Capitain, O.; Ramos, M.; et al. Phase III trial of sunitinib in combination with capecitabine versus capecitabine monotherapy for the treatment of patients with pretreated metastatic breast cancer. J. Clin. Oncol. 2013, 31, 2870-2878.

162. Barrios, C.H.; Liu, M.-C.; Lee, S.C.; Vanlemmens, L.; Ferrero, J.-M.; Tabei, T.; Pivot, X.; Iwata, H.; Aogi, K.; Lugo-Quintana, R.; et al. Phase III randomized trial of sunitinib versus capecitabine in patients with previously treated HER2-negative advanced breast cancer. Breast Cancer Res. 
Treat. 2010, 121, 121-131.

163. Bergh, J.; Bondarenko, I.M.; Lichinitser, M.R.; Liljegren, A.; Greil, R.; Voytko, N.L.; Makhson, A.N.; Cortes, J.; Lortholary, A.; Bischoff, J.; et al. First-line treatment of advanced breast cancer with sunitinib in combination with docetaxel versus docetaxel alone: results of a prospective, randomized phase III study. J. Clin. Oncol. 2012, 30, 921-929.

164. Buchbinder, E.I.; Sosman, J.A.; Lawrence, D.P.; McDermott, D.F.; Ramaiya, N.H.; Van den Abbeele, A.D.; Linette, G.P.; Giobbie-Hurder, A.; Hodi, F.S. Phase 2 study of sunitinib in patients with metastatic mucosal or acral melanoma. Cancer 2015, 121, 4007-4015.

165. Decoster, L.; Broek, I.; Neyns, B.; Majois, F.; Baurain, J.F.; Rottey, S.; Rorive, A.; Anckaert, E.; De Mey, J.; De Brakeleer, S.; et al. Biomarker analysis in a phase II study of sunitinib in patients with advanced melanoma. Anticancer Res. 2015, 35, 6893-6899.

166. Gong, H.Z.; Zheng, H.Y.; Li, J. The clinical significance of KIT mutations in melanoma: a metaanalysis. Melanoma Res. 2018, 28, 259-270.

167. Meng, D.; Carvajal, R.D. KIT as an oncogenic driver in melanoma: an update on clinical development. Am. J. Clin. Dermatol. 2019, 20, 315-323.

168. Murakami, A.; Mori, T.; Sakai, H.; Murakami, M.; Yanai, T.; Hoshino, Y.; Maruo, K. Analysis of KIT expression and KIT exon 11 mutations in canine oral malignant melanomas. Vet. Comp. Oncol. 2011, 9, 219-224.

169. Gomes, J.; Queiroga, F.L.; Prada, J.; Pires, I. Study of c-kit immunoexpression in canine cutaneous melanocytic tumors. Melanoma Res. 2012, 22, 195-201.

170. Chu, P.-Y.; Pan, S.-L.; Liu, C.-H.; Lee, J.; Yeh, L.-S.; Liao, A.T. KIT gene exon 11 mutations in canine malignant melanoma. Vet. J. 2013, 196, 226-230.

171. Newman, S.J.; Jankovsky, J.M.; Rohrbach, B.W.; LeBlanc, A.K. c-kit expression in canine mucosal melanomas. Vet. Pathol. 2011, 49, 760-765.

172. Giuliano, A.; Dobson, J. Prospective clinical trial of masitinib mesylate treatment for advanced stage III and IV canine malignant melanoma. J. Small Anim. Pract. 2020, 61, 190-194.

173. Londhe, P.; Gutwillig, M.; London, C. Targeted therapies in veterinary oncology. Vet. Clin. North Am. Small Anim. Pract. 2019, 49, 917-931.

174. Cekanova, M.; Rathore, K. Animal models and therapeutic molecular targets of cancer: utility and limitations. Drug Des. Devel. Ther. 2014, 8, 1911-1921.

175. Paoloni, M.; Mazcko, C.; Selting, K.; Lana, S.; Barber, L.; Phillips, J.; Skorupski, K.; Vail, D.; Wilson, H.; Biller, B.; et al. Defining the pharmacodynamic profile and therapeutic index of NHSIL12 immunocytokine in dogs with malignant melanoma. PLoS One 2015, 10, e0129954.

176. Fürdös, I.; Fazekas, J.; Singer, J.; Jensen-Jarolim, E. Translating clinical trials from human to veterinary oncology and back. J. Transl. Med. 2015, 13, 265-271.

177. Hünig, T. The storm has cleared: Lessons from the CD28 superagonist TGN1412 trial. Nat. Rev. Immunol. 2012, 12, 317-318.

178. Liu, D.; Xiong, H.; Ellis, A.E.; Northrup, N.C.; Dobbin, K.K.; Shin, D.M.; Zhao, S. Canine spontaneous head and neck squamous cell carcinomas represent their human counterparts at the molecular level. PLoS Genet. 2015, 11, e1005277.

179. Amini, P.; Nassiri, S.; Malbon, A.; Markkanen, E. Differential stromal reprogramming in benign and malignant naturally occurring canine mammary tumours identifies disease-modulating stromal components. Sci. Rep. 2020, 10, 5506. 



\section{CHAPTER 5}

\section{Nanobody-targeted photodynamic therapy for the treatment of feline oral carcinoma: a step towards translation to the veterinary clinic}

Irati Beltrán Hernández ${ }^{1,2}$, Guillaume C.M. Grinwis ${ }^{3}$, Paul M.P. van Bergen en Henegouwen ${ }^{2}$, Wim E. Hennink ${ }^{1}$, Erik Teske ${ }^{4}$, Jan W. Hesselink ${ }^{4}$, Sebastiaan A. van Nimwegen ${ }^{4}$, Jan A. Mol ${ }^{4}$ and Sabrina Oliveira ${ }^{1,2}$

\footnotetext{
${ }^{1}$ Pharmaceutics, Department of Pharmaceutical Sciences, Faculty of Science, Utrecht University, Utrecht, the Netherlands

${ }^{2}$ Cell Biology, Neurobiology and Biophysics, Department of Biology, Faculty of Science, Utrecht University, Utrecht, the Netherlands

${ }^{3}$ Department of Biomedical Health Sciences, Faculty of Veterinary Medicine, Utrecht University, Utrecht, the Netherlands

${ }^{4}$ Department of Clinical Sciences, Faculty of Veterinary Medicine, Utrecht University, Utrecht, the Netherlands
} 


\begin{abstract}
Nanobody-targeted photodynamic therapy (NB-PDT) has been developed as a potent and tumor-selective treatment, using nanobodies (NBs) to deliver a photosensitizer (PS) specifically to cancer cells. Upon local light application, reactive oxygen species are formed and consequent cell death occurs. NB-PDT has preclinically shown evident success and we now aim to treat cats with oral squamous cell carcinoma (OSCC), which has very limited curative options so far and is regarded as a natural model of human head and neck SCC. Immunohistochemistry of feline OSCC tissue confirmed that the epidermal growth factor receptor (EGFR) is a relevant target with expression in cancer cells and not in the surrounding stroma. Three feline OSCC cell lines were employed together with a well-characterized human cancer cell line (HeLa), all with similar EGFR expression, and a low EGFR-expressing human cell line (MCF7), mirroring the EGFR expression level in the surrounding mucosal stroma. $\mathrm{NB}_{\mathrm{A}}$ was identified as a NB binding human and feline EGFR with comparable high affinity. This NB was developed into $\mathrm{NiBh}$, a NB-PS conjugate with high PS payload able to effectively kill feline OSCC and HeLa cell lines, after illumination. Importantly, the specificity of NB-PDT was confirmed in co-cultures where only the feline OSCC cells were killed while surrounding MCF7 cells were unaffected. Altogether, NiBh can be used for NB-PDT to treat feline OSCC and further advance NB-PDT towards the human clinic.
\end{abstract}




\section{Introduction}

Nanobody-targeted photodynamic therapy (NB-PDT) has been developed in the last 6 years as a highly tumor-selective treatment [1-8]. This strategy makes use of nanobodies (NBs) to deliver a photosensitizer (PS) in the form of NB-PS conjugates specifically to tumor cells. NBs are the smallest naturally-derived antigen-binding fragments $(\sim 16 \mathrm{kDa})$, which constitute the variable domain of heavy-chain antibodies occurring in Camelids and cartilaginous fish [9]. Photosensitizers are light-activatable compounds that, upon accumulation in the tumor, can be excited by light of a particular wavelength locally applied at the tumor site, leading to the formation of reactive oxygen species and consequent cell death $[10,11]$. In this respect, two levels of specificity, i.e. tumor targeting via NBs and local PS activation, make it possible to effectively kill cancer cells, while sparing surrounding healthy tissue.

NB-PDT has proven to be very specific in the in vitro setting for a wide range of membrane receptors, including epidermal growth factor receptor (EGFR) [1,2], human epidermal growth factor receptor 2 (HER2) [3], US28 G protein-coupled receptor (GPCR) [4] and the hepatocyte growth factor receptor (c-Met) [5]. By taking advantage of the overexpression of such membrane proteins in cancer cells (or their absence in normal cells, as for US28), the greater association of PS with cancer cells combined with local illumination leads to cancer-specific cytotoxicity. In vivo, NB-PDT induces extensive damage to the tumor [2] and causes significant tumor regression after one single treatment session [3]. Permanent vascular effects including vasoconstriction, reduced perfusion and leakage have also been observed in the tumor area after NB-PDT [6]. Furthermore, the first indications of immunogenic cell death induced by NB-PDT have been recently reported [7], which suggests that antitumor immunity can be triggered [12].These three antitumor mechanisms, i.e. direct tumor cell killing, tumor-associated vasculature effects and antitumor immunity, have been described for both conventional PDT [10] and antibody-targeted PDT [13]. Nevertheless, preclinical data indicate that NB-PDT offers advantages over these two PDT approaches.

Conventional PDT is nowadays used for many oncological indications, such as basal cell carcinoma, oesophageal cancer and head and neck squamous cell carcinoma (HNSCC) [10]. Despite good outcomes, the passive accumulation of the (usually hydrophobic) PS in the tumor generally leads to light application 24-72 hours after injection, and the slow tissue clearance of the PS causes skin photosensitivity for days/weeks after treatment $[10,11]$. Active targeting is achieved with antibody-targeted PDT, a strategy under evaluation in a phase III clinical trial for the treatment of HNSCC (NCT03769506). Here, the anti-EGFR antibody cetuximab is conjugated to the watersoluble PS IRDye700DX. However, the use of such a relatively large antibody with long circulation times still leaves several points of improvement, especially regarding tumor distribution of the conjugate and its slow clearance. The benefits of NB-PDT, which also uses IRDye700DX as PS, are the small size and high affinity of NBs which now guide the pharmacokinetics of the PS [2,3]. These properties are responsible for the observed rapid and homogeneous accumulation of the conjugate in the tumor. Thus, illumination shortly after injection is feasible, which substantially improves the logistics of this treatment modality. Moreover, due to their small size, these conjugates are rapidly cleared from circulation by the kidneys, which potentially reduces the skin phototoxicity period after treatment. Altogether, the promise of NB-PDT is evident, and this prompts the next logical step of further translation to the clinic.

In view of clinical translation, EGFR-targeted NB-PDT has already been proven to be effective and selective using patient-derived HNSCC organoids and corresponding normal tissue organoids derived from the same patient [8]. Importantly, low/moderate 
EGFR levels were found on this patient cancer material, in comparison to common HNSCC cell lines. Although this led to a less potent effect of NB-PDT, tumor organoids were still killed, while normal organoids were unaffected. This highlights the importance of assessing treatment efficacy in the context of clinically relevant target expression. As the next step, we have directed ourselves to the possible application in oncological animal patients aiming to treat spontaneous tumors with high biological relevance; in particular, cats with oral squamous cells carcinoma (OSCC). From all feline tumors, 10\% of these occur in the oral cavity, being SCC the most abundant among these oral malignant tumors [14]. OSCC are fast-growing tumors and locally invasive, but they have a low metastatic potential. Limited success in the treatment of such tumors has been achieved so far by radiotherapy, chemotherapy, surgery or a combination of these [15,16]. For most therapies, the medium survival time after diagnosis is around 3 months, where euthanasia is usually the only option due to the poor quality of life and limited options for a successful treatment [17]. The poor response of feline OSCC to current treatments, the superficial nature of these lesions and infrequent metastasis account for the decision to investigate NB-PDT as a new treatment for these patients. In addition, feline OSCC shares similar pathogenesis, tumor biology and molecular markers with human head and neck squamous cell carcinoma (HNSCC) [18], making it a very relevant choice to facilitate and accelerate translation to human patients. The fact that conventional PDT is already part of the arsenal to combat HNSCC in the clinic, reinforces the choice to treat feline oral cancer with (targeted) PDT [10]. As a molecular target to be used by NB-PDT in feline OSCC, EGFR is a relevant choice due to its overexpression in HNSCC [19] as well as feline OSCC [20,21]. Furthermore, the high homology of this protein between both species ( $92 \%$, NCBI BLAST) broadens the possibilities to find cross-reactive NBs to the human and feline receptor to allow a smoother transition to the human clinic.

In this study, we describe the in vitro characterization of a species cross-reactive NB and its use for EGFR-targeted NB-PDT on a panel of feline OSCC cell lines, i.e. SCCF1, SCCF2 and SCCF3 cells. Bearing in mind the translation to the human clinic, a human cancer cell line (HeLa) with clinically relevant target expression was taken along, with EGFR levels in the range of human head and neck cancers [8]. With the goal to demonstrate the specificity of this approach, a major advantage of NB-PDT, a human low EGFR-expressing cell line was included in the study (MCF7) as representation of the stroma cells of the normal oral mucosa [22,23]. The fact that moderate membrane EGFR levels are expected in the clinical setting [8,23], together with the successful results obtained so far in clinical trials with antibody-PS conjugates bearing high PS to antibody ratios (NCT02422979) [24,25], led us to develop a NB-PS conjugate with high payload (average of 2.5 PS molecules per NB), named NiBh. This attractive conjugate maintained high affinity across species and target-specific potency when used for NB-PDT, highlighting its potential to treat cats with oral carcinoma and further advance the application of NB-PDT in the clinic.

\section{Materials and Methods}

\section{Cell lines and culture}

The human cervical adenocarcinoma cell line HeLa and the human mammary adenocarcinoma cell line MCF7 were purchased from ATCC (ATCC CCL-2 and HTB22). The feline oral squamous cell carcinoma cell lines SCCF1, SCCF2 and SCCF3 were kindly provided by Dr. Rosol (Ohio University). SCCF1 derives from a laryngeal SCC, SCCF2 from a bone-invasive gingival SCC and SCCF3 from a lingual SCC [26,27]. All 
cells were cultured in Dulbecco's Modified Eagle's Medium (DMEM) with high glucose and ultraglutamine 1 (Lonza, Basel, Switzerland) supplemented with 10\% foetal bovine serum (FBS) (Sigma-Aldrich, Zwijndrecht, the Netherlands), $100 \mathrm{U} / \mathrm{mL}$ penicillin and $100 \mu \mathrm{g} / \mathrm{mL}$ streptomycin (Sigma-Aldrich). Cells were cultured at $37{ }^{\circ} \mathrm{C}$ and $5 \% \mathrm{CO}_{2}$.

\section{Nanobody and conjugation to fluorophore/photosensitizer}

$\mathrm{NB}_{\mathrm{A}}$ was identified from a previous panel of NBs selected against the extracellular domain of human EGFR described in [28], particularly from a screen of NBs that inhibit EGF binding to EGFR. NBA was produced and purified from the periplasmic fraction of E. Coli as previously described [29].

For fluorescence detection, NBA was conjugated to the fluorophore Alexa Fluor 647 NHS ester (Invitrogen, Carlsbad, CA, USA) following the manufacturer's protocol, yielding a degree of conjugation (DOC), i.e. PS to NB ratio, of 1 . Briefly, $\mathrm{NB}_{\mathrm{A}}$ in PBS was incubated with the fluorophore for 2 hours at room temperature, in a molar ratio of 1 to 4 . Thereafter, free fluorophore was removed by size exclusion chromatography using three consecutive Zeba Spin Desalting Columns (Thermo Fisher Scientific, Perbio Science Nederland, Etten-Leur, the Netherlands).

For NB-PDT assays, NBA was conjugated to the PS IRDye700DX (LI-COR, Biosciences, Lincoln, NE, USA). For this, the provider's protocol was followed, controlling the conjugation conditions to achieve NB-PS conjugates with DOC $0.5,1$ and 2.5, the latter referred to as NiBh. Free PS was removed using four consecutive Zeba Spin Desalting Columns. Further characterization of the NB-PS conjugates was performed as described in the Supplementary Materials.

\section{Immunohistochemistry}

Paraffin-embedded tissue blocks of feline OSCC were obtained from the archive of the Pathology division of the Veterinary Faculty at Utrecht University. Ten cases which contained OSCC as well as normal oral epithelium within the same tissue block were selected. For immunohistochemistry, $4 \mu \mathrm{m}$-thick tissue sections were mounted onto slides, deparaffinized and rehydrated. Antigen retrieval took place for 4 minutes at $37^{\circ} \mathrm{C}$ with proteinase K (DAKO, Amstelveen, the Netherlands, cat no. S3020). Endogenous peroxidase was blocked with dual endogenous enzyme block (DAKO, S2003) for 10 minutes at room temperature and tissue was then blocked with PBS $+0.1 \%$ Tween $(\mathrm{PBST})+10 \%$ BSA for 1 hour at room temperature. Tissue was incubated overnight at $4{ }^{\circ} \mathrm{C}$ with mouse anti-EGFR antibody (Thermo Fisher Scientific, MA5-13269) diluted $1: 100$ in PBST $+1 \%$ BSA. This primary antibody is a species cross-reactive antibody known to bind EGFR in dog, human, mouse, sheep, non-human primate and cat. Its detection was performed with the kit Envision + System-HRP anti-mouse (DAKO, K4401) for 30 minutes at room temperature. Staining was visualized by applying DAB chromogen (DAKO, K3468) and reaction stopped after 4 minutes. Slides were counterstained with hematoxylin. Canine skin was used as positive control tissue. As negative control, normal mouse IgG (Santa Cruz Biotechnology, Heidelberg, Germany, cat no. sc-2025) was used instead of the primary antibody. Immunoreactivity was evaluated as the product of percentage of positive tumor cells $(1=<10 \%, 2=10-30 \%$, $3=31-60 \%$, or $4=>60 \%)$ and staining intensity $(1=$ low, $2=$ moderate, $3=$ high $)$, as in previous publications $[20,30]$. Scores $\geq 2$ were considered positive. To quantify staining intensity, ImageJ was used for deconvolution of the DAB color spectra and the optical density recorded in the regions of interest (tumor nests or basal layer of normal epithelium). Outcome was verified by an experienced veterinary pathologist (Guillaume C.M. Grinwis). 
For immunohistochemistry with fluorescence detection, slides were subjected to the same steps as explained above. However, after incubation with the primary antibody, goat anti-mouse Alexa 555 (Invitrogen, A21424) was used as the secondary antibody $(1: 200$ in PBST $+1 \%$ BSA) for 1 hour at room temperature. Alternatively, to detect EGFR with $\mathrm{NB}_{\mathrm{A}}$ instead, $10 \mathrm{nM}$ of directly labeled $\mathrm{NB}_{\mathrm{A}}$-Alexa 647 were incubated overnight at $4{ }^{\circ} \mathrm{C}$. Slides were counterstained with DAPI (Roche, Basel, Switzerland). Images were taken with a Confocal Laser Scanning Microscope (Carl Zeiss Microscopy GmbH, Germany, LSM700) using a plan-apochromat 63x/1.40 Oil DIC and 20x/0.8 M27 objectives.

\section{Binding assay}

To assess the apparent binding affinity $\left(\mathrm{K}_{\mathrm{D}}\right)$ of NBs on EGFR-expressing human and feline cells, 10.000 cells per well were seeded in 96 -well plates and incubated at $37^{\circ} \mathrm{C}$. The next day, cells were incubated for 2 hours at $4{ }^{\circ} \mathrm{C}$ with a concentration range of NB $(0.2-100 \mathrm{nM})$ in binding medium (DMEM without phenol red, supplemented with 25 $\mathrm{mM}$ HEPES and $1 \% \mathrm{BSA}, \mathrm{pH}$ 7.2). Unbound NB was washed off and cells fixed with $4 \%$ PFA (Merck, Haarlem, the Netherlands) for 10 minutes at room temperature. Bound NB was detected by incubating with rabbit anti-VHH antibody (QVQ, Utrecht, the Netherlands, cat no. QE19) for 1 hour at room temperature (1:1000 in PBS + 1\% BSA) followed by goat anti-rabbit IRDye $800 \mathrm{CW}$ for 1 hour at room temperature $11: 2000$ in PBS $+1 \%$ BSA). Fluorescence at $800 \mathrm{~nm}$ was detected with an Odyssey infrared scanner (LI-COR). To assess the apparent binding affinity of the conjugates $\mathrm{NB}_{\mathrm{A}}-\mathrm{PS}$ and $\mathrm{NiBh}$, the same procedure was followed, but plates were directly scanned at $700 \mathrm{~nm}$ after washing off the unbound conjugate. Data was analyzed with GraphPad Prism software (GraphPad, La Jolla, CA, USA) and $\mathrm{K}_{\mathrm{D}}$ values determined using a non-linear fit with onesite specific binding.

The binding of NiBh was also assessed in the presence of EGF. For this, $10 \mathrm{nM}$ of $\mathrm{NiBh}$ was incubated with the cells in the absence or presence of an equimolar concentration of EGF or a 10x molar excess of EGF (R\&D Systems, Minneapolis, MN, USA, cat no. 236-EG).

\section{Flow cytometry}

Cells were added in a U-bottom 96 well-plate $\left(10^{5}\right.$ cells per well), washed once with PBS $+1 \%$ BSA and incubated for 45 minutes at $4{ }^{\circ} \mathrm{C}$ with $20 \mathrm{nM}$ of mouse anti-EGFR antibody. After washing, secondary antibody goat anti-mouse Alexa 488 diluted 1:200 was added and incubated with the cells for 30 minutes at $4{ }^{\circ} \mathrm{C}$. Alternatively, to detect EGFR with $\mathrm{NB}_{\mathrm{A}}$ instead, $40 \mathrm{nM}$ of labeled $\mathrm{NB}_{\mathrm{A}}$-Alexa 647 was incubated with the cells for 30 minutes at $4{ }^{\circ} \mathrm{C}$. Unstained controls and samples stained with secondary antibody only were taken along for each cell line. Measurements were performed with a FACS Canto II (BD Biosciences, San Jose, CA, USA) and further analyzed with FlowLogic software (Inivai Technologies). The fluorescence intensity of both fluorophores was normalized to compare both in a single graph.

\section{Immunofluorescence on cells}

Ten thousand cells per well were seeded in 16 wells Lab-Tek Chamber Slides (Thermo Fisher Scientific, 178599) and incubated at $37^{\circ} \mathrm{C}$. The next day, cells were washed with binding medium and co-stained with $20 \mathrm{nM}$ mouse anti-EGFR antibody and $40 \mathrm{nM} \mathrm{NB}_{\mathrm{A}}-$ Alexa 647 in binding medium. Incubation took place for 1.5 hours at $4{ }^{\circ} \mathrm{C}$ and, subsequently, the cells were fixed with $4 \%$ PFA for 10 minutes at room temperature. The secondary antibody goat anti-mouse Alexa 488 (Invitrogen, A11029) was added for 1 
hour at room temperature (1:200 in PBS $+1 \%$ BSA). Cells were stained with DAPI and imaged with a confocal microscope.

\section{Nanobody-targeted photodynamic therapy}

NB-PDT was performed as previously described [1]. Briefly, cells were seeded in 96well plates $\left(10.000\right.$ cells/well) one day before the assay and incubated at $37^{\circ} \mathrm{C}$. The next day, cells were incubated with a concentration range of NB-PS conjugate $(0.78-100 \mathrm{nM})$ for 30 minutes at $37{ }^{\circ} \mathrm{C}$ in PDT medium, i.e. DMEM without phenol red and L-glutamine (Lonza) supplemented with 10\% FBS and antibiotics. Unbound conjugate was washed off and the plate was scanned with the Odyssey scanner at $700 \mathrm{~nm}$ to detect association of the conjugate with cells. Thereafter, cells were illuminated with $7 \mathrm{~mW} / \mathrm{cm}^{2}$ for 59 minutes $\left(25 \mathrm{~J} / \mathrm{cm}^{2}\right)$ using a $690 \mathrm{~nm}$ laser (Modulight ML7700, Tampere, Finland). Fluence rate was monitored with an Orion/PD optometer (Ophir Optronics, Jerusalem, Israel). After illumination, the plates were placed back at $37^{\circ} \mathrm{C}$.

NB-PDT was also performed with an excess of $\mathrm{NB}_{\mathrm{A}}$, i.e. under competing conditions, with some minor adjustments of the above-mentioned protocol. Briefly, before adding $\mathrm{NiBh}(50 \mathrm{nM})$, cells were pre-incubated for 10 minutes at $37^{\circ} \mathrm{C}$ with $10 \mathrm{x}$ or 50x molar excess of $\mathrm{NB}_{\mathrm{A}}$. The unconjugated $\mathrm{NB}$ was present as well during the incubation with $\mathrm{NiBh}$. In a different experiment, NB-PDT was performed in the presence of $25 \mathrm{mM}$ or $50 \mathrm{mM}$ sodium azide, as a quencher of singlet oxygen [31]. In this case, sodium azide was added to the cells only during the illumination time.

\section{Cell viability and cell death after NB-PDT}

One day after NB-PDT, cells were incubated with Alamar Blue reagent (Bio-Rad, Hercules, CA, USA) to assess viability, according to the protocol of the manufacturer. Fluorescence was measured with a FLUOstar Optima microplate reader (BMG Labtech, Ortenberg, Germany) and results expressed as cell viability in percentage relative to untreated cells. The median lethal dose (LD50), i.e. concentration of conjugate to achieve $50 \%$ of cell death, was determined using GraphPad Prism software with a log (inhibitor) vs normalized response fit.

Alternatively, 2 and 24 hours after NB-PDT, live and dead cells were distinguished by staining with calcein AM (Invitrogen) and propidium iodide (PI) (Invitrogen) at a final dilution of 1:2000 and 1:1000, respectively, for 10 minutes at $37^{\circ} \mathrm{C}$. Cells were imaged with an EVOS microscope (Thermo Fisher Scientific) using transmitted light, a GFP cube for calcein AM, and a RFP cube for PI.

\section{Co-cultures and specificity assays}

SCCF2 and MCF7 cells were brought in suspension and labeled with the cell tracking dyes ViaFluor 405 and ViaFluor 488 (Biotium, Amsterdam, the Netherlands, cat no. 30068 and 30086), respectively, according to the provider's protocol. Both cell lines were mixed in a ratio 1:1, seeded in 16 wells Lab-Tek Chamber Slides (Thermo Fisher Scientific) at a density of 10.000 cells per well and cultured at $37^{\circ} \mathrm{C}$. Cells were used the next day for further assays.

The co-cultures were incubated with $50 \mathrm{nM}$ of $\mathrm{NB}_{\mathrm{A}}$-Alexa 647 in culture medium for 30 minutes at $37^{\circ} \mathrm{C}$. Alternatively, $\mathrm{NB}_{\mathrm{A}}$-Alexa 647 was incubated for 1 hour at $4{ }^{\circ} \mathrm{C}$ in binding medium. Thereafter, cells were fixed, stained with DAPI and imaged with a confocal microscope.

In a different assay, co-cultures were treated with NB-PDT using $50 \mathrm{nM}$ of NiBh. Cells were placed back in the incubator and, 2 hours after illumination, cells were stained with PI (1:1000) and directly imaged with a confocal microscope. 


\section{Results}

\section{EGFR is expressed in feline oral squamous cell carcinoma and in normal oral epithelium}

As EGFR has been described to be overexpressed in feline OSCC [20,21], immunohistochemistry was performed on 10 cases of feline OSCC to investigate the expression of this protein in neoplastic cells and to elucidate its presence in cells of the surrounding normal oral mucosa (epithelium and stroma). EGFR presence, with predominant cell membrane localization, was detected in neoplastic cells forming nests and trabecula, and not found to be expressed in the surrounding stroma cells (Figure 1a, right). On the other hand, membranous EGFR expression was also detected in the normal adjacent oral epithelium, more prominently in the basal cell layer and decreasing towards the outer, more differentiated epithelial layers (Figure 1a, left). Interestingly, a similar pattern of differentiation and loss of EGFR expression was observed towards the core of large neoplastic nests which typically contain more differentiated neoplastic cells that can even show keratinization. For all 10 studied cases, EGFR positivity in the basal epithelial layer of preexisting normal epithelium was in the same range as in the neoplastic nests (Figure 1b). Accordingly, nine of the ten investigated cases expressed intermediate to high EGFR levels in the neoplastic nests, but EGFR was not overexpressed compared to the expression observed in the basal layer of the epithelium (Figure 1c). Altogether, these observations support that EGFR is a relevant target due to its expression in tumor cells and its absence in stroma.

(a)

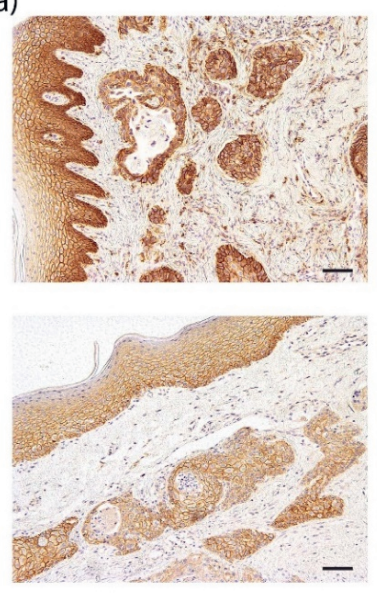

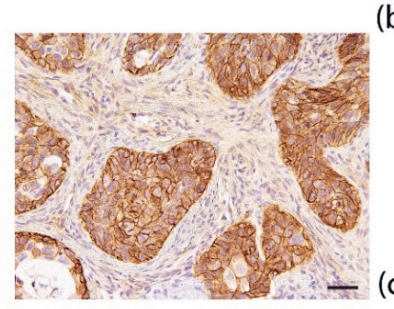

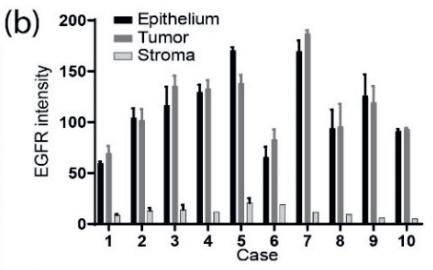

(c)

\begin{tabular}{|c|c|c|c|}
\hline \multirow{2}{*}{ Case } & \multicolumn{2}{|c|}{$\begin{array}{c}\text { Immunoreactivity } \\
\text { score (0-12) }\end{array}$} & \multirow{2}{*}{$\begin{array}{c}\text { EGFR } \\
\text { expression }\end{array}$} \\
\cline { 2 - 3 } & Tumor & Epithelium & \\
\hline $\mathbf{1}$ & 4 & 4 & weak \\
$\mathbf{2}$ & 8 & 8 & intermediate \\
$\mathbf{3}$ & 12 & 12 & strong \\
$\mathbf{4}$ & 12 & 12 & strong \\
$\mathbf{5}$ & 12 & 12 & strong \\
$\mathbf{6}$ & 8 & 8 & intermediate \\
$\mathbf{7}$ & 12 & 12 & strong \\
$\mathbf{8}$ & 8 & 8 & intermediate \\
$\mathbf{9}$ & 8 & 8 & intermediate \\
$\mathbf{1 0}$ & 8 & 8 & intermediate \\
\hline
\end{tabular}

Figure 1 EGFR expression in feline OSCC and surrounding tissue. Immunohistochemistry was performed in 10 cases of OSCC with normal adjacent oral epithelium. (a) Representative images are shown of neoplastic nests compared to normal neighbouring epithelium (left) and neoplastic nests surrounded by stroma (right), for two cases with strong (top) and intermediate (bottom) EGFR expression. Scale bar, $100 \mu \mathrm{m}$ (left images) and $50 \mu \mathrm{m}$ (right images). (b) Quantification of the EGFR signal in neoplastic nests (Tumor), mucosal stroma (Stroma) and basal layer of the adjacent oral epithelium (Epithelium) per case. (c) Immunoreactivity score and classification of each case based on EGFR intensity calculated in $\mathrm{B}$ and $\%$ of positive tumor cells. 


\section{$\mathrm{NB}_{\mathrm{A}}$ is a species cross-reactive NB targeting EGFR in human and feline cells}

Having identified EGFR as a promising molecular target in feline oral carcinoma, we first confirmed the presence of membrane EGFR on three feline OSCC cell lines (SCCF1, SCCF2 and SCCF3), in comparison to two well-characterized human cancer cell lines (HeLa and MCF7) (Figure S1). This was performed with flow cytometry using a commercial EGFR-targeting, species cross-reactive antibody. Thereafter, the binding affinity of a panel of NBs originally selected against human EGFR (with binding affinities, $\mathrm{K}_{\mathrm{D}},<10 \mathrm{nM}$ ) was assessed on SCCF1 cells. From this NB panel, NBA was identified as the most promising candidate due to its high binding affinity $\left(\mathrm{K}_{\mathrm{D}} \sim 0.44 \mathrm{nM}\right)$ for feline cells (Figure 2a). To further investigate the species cross-reactivity of $\mathrm{NB}_{\mathrm{A}}$ and its EGFR specificity, its binding to the three feline OSCC cell lines and the two human cancer cell lines was evaluated using flow cytometry and immunofluorescence. As a reference, the commercial EGFR-targeting antibody was used. $\mathrm{NB}_{\mathrm{A}}$ was first conjugated to the fluorophore Alexa 647 (with binding affinity comparable to $\mathrm{NB}_{\mathrm{A}}$, data not shown) to enable direct detection in the assays. The three feline OSCC cell lines had EGFR levels in the range of HeLa cells, while the fluorescence signal was minimal on the low EGFRexpressing MCF7 cells (Figure 2b, 2c). The same EGFR expression trend and pattern on the different cell lines was detected by the reference antibody, indicating the capability of $\mathrm{NB}_{\mathrm{A}}$ to bind both human and feline EGFR (Figure 2b, 2c). Further supporting this, EGFR was also detected by $\mathrm{NB}_{\mathrm{A}}$ (and the commercial antibody) on feline OSCC tissue (Figure 2d), predominantly at the basal epithelium and neoplastic nests.

\section{$\mathrm{NiBh}$, a $\mathrm{NB}_{\mathrm{A}}-\mathrm{PS}$ conjugate with high payload, is a potent and specific agent for EGFR-targeted PDT}

$\mathrm{NB}_{\mathrm{A}}$ was conjugated to the PS aiming to obtain conjugates with a different degree of conjugation (DOC), i.e. PS to NB ratios, in order to increase the PS density on tumor cells and, thus, cytotoxicity upon illumination. Accordingly, three conjugates were synthesized with $0.5,1$ or 2.5 PS molecules per NB molecule, named $\mathrm{NB}_{\mathrm{A}-\mathrm{PS}}(0.5), \mathrm{NB}_{\mathrm{A}}-\mathrm{PS}(1)$ and $\mathrm{NiBh}$, respectively. The conjugates were characterized in terms of purity by SDS-PAGE and the PS absorbance spectra was acquired, showing that the absorbance properties of the PS were not affected when conjugated to the NB, regardless of the DOC (Figure S2). Although the apparent binding affinities of the conjugates to both human and feline cells were slightly affected with an increasing degree of PS modification, the affinities still remained in the low nanomolar range (Figure 3a). Furthermore, the specificity of the conjugates for (feline) EGFR was verified by using EGFR knockdown SCCF1 cells, which resulted in a considerably reduced binding of the conjugate (Figure S3). When employing these conjugates for NB-PDT, it is evident that the use of NiBh resulted in the highest fluorescence (or density) of PS associated with the cells (Figure 3b) and, after illumination, $\mathrm{NiBh}$ was the only conjugate that induced significant cytotoxicity to the feline cells (Figure 3c). 
(a)

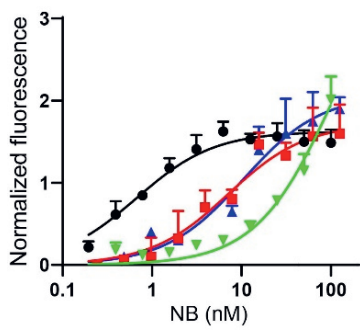

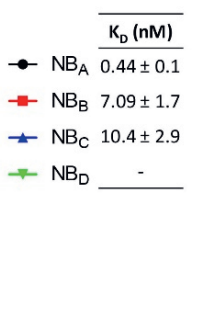

(b)

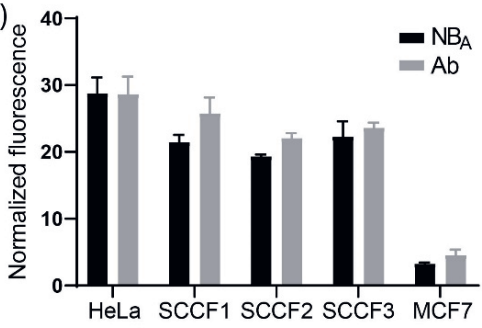

(c)
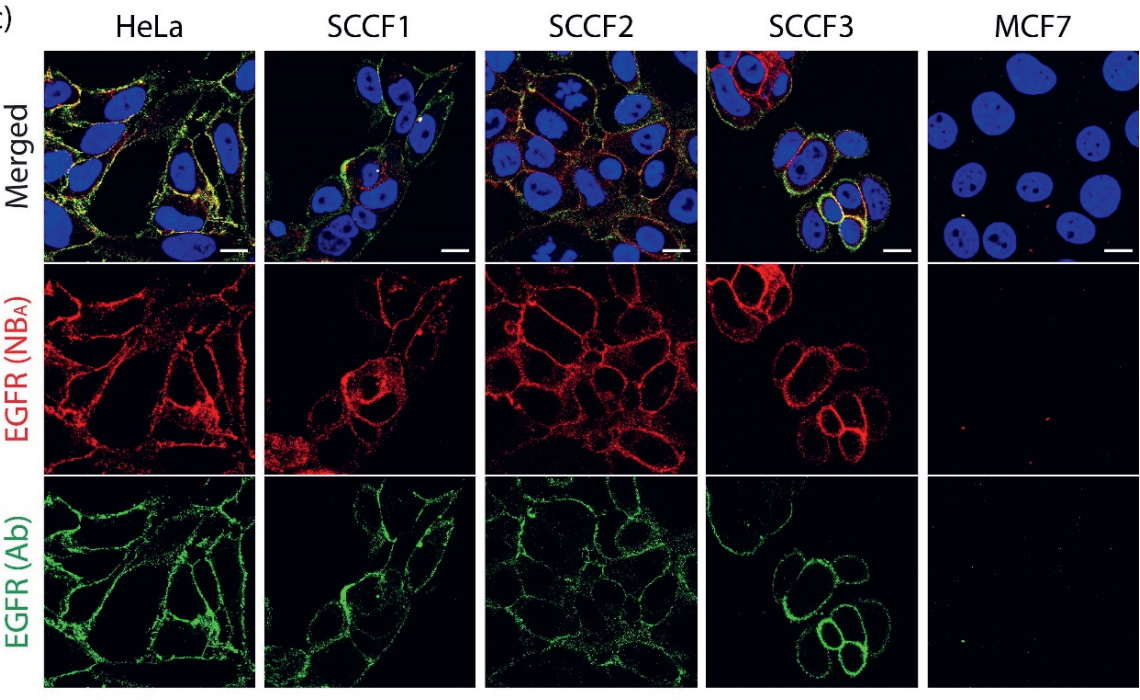

(d)
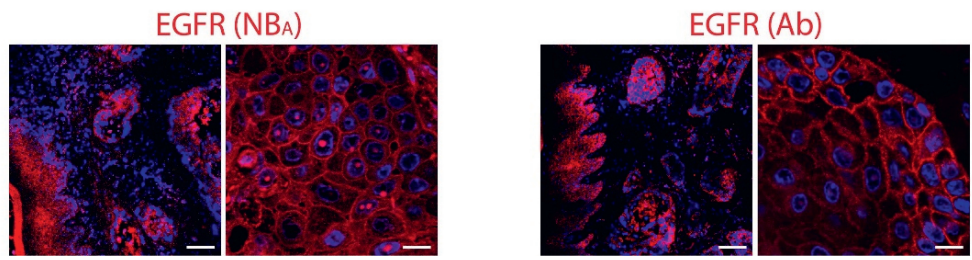

Figure 2 EGFR expression on human and feline cells and tissue detected by $\mathrm{NB}_{\mathrm{A}}$. (a) A binding assay was performed with representative NBs, previously selected against human EGFR, using feline SCCF 1 cells. The graph displays the binding curves of several NBs to SCCF1 cells. The apparent binding affinities $\left(\mathrm{K}_{\mathrm{D}}\right)$ of the different NBs are shown. (b) and (c) Membrane EGFR was detected on three feline OSCC cell lines (SCCF1, SCCF2 and SCCF3) and two human cancer cell lines (HeLa and MCF7). (b) Membrane EGFR levels measured by flow cytometry using a commercial anti-EGFR antibody or $\mathrm{NB}_{\mathrm{A}}$-Alexa 647 , expressed as median fluorescence intensity (MFI) and normalized. (c) Confocal microscope images of each cell line co-stained for membrane EGFR using a commercial species cross-reactive antibody (green, middle panel) and $\mathrm{NB}_{\mathrm{A}}$-Alexa 647 (red, bottom panel). Nuclei were stained with DAPI (blue). Merged images are shown on the top panels. Scale bar, $15 \mu \mathrm{m}$. (d) Feline OSCC tissue sections stained with $\mathrm{NB}_{\mathrm{A}}$-Alexa 647 (left) or a commercial anti-EGFR antibody (right). Images show an overview of the OSCC tissue including adjacent normal oral epithelium, and a close-up of the tumor cells. Scale bar, $100 \mu \mathrm{m}$ (left images) and $15 \mu \mathrm{m}$ (right images). 
(a)
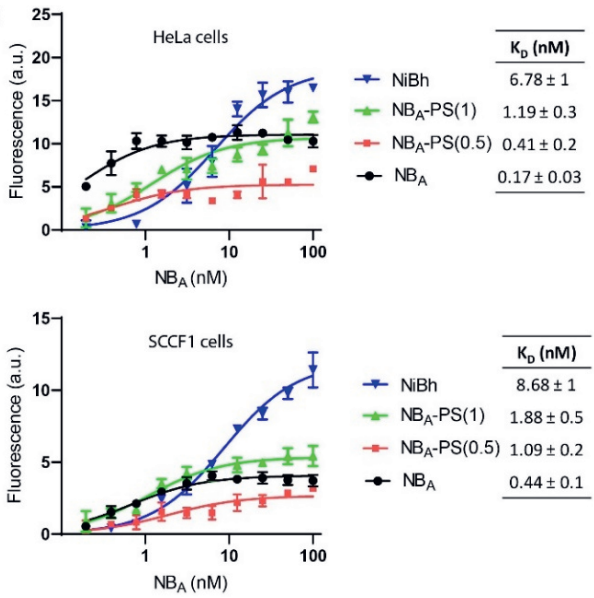

(b)

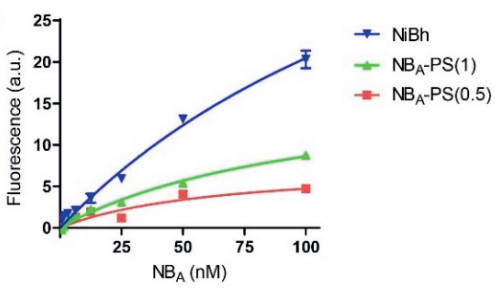

(c)

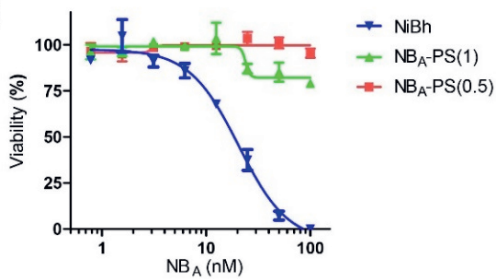

Figure 3 Binding affinity and potency of different $\mathrm{NB}_{\mathrm{A}}-\mathrm{PS}$ conjugates. (a) A binding assay was performed with $\mathrm{NB}_{\mathrm{A}}, \mathrm{NB}_{\mathrm{A}}-\mathrm{PS}(0.5), \mathrm{NB}_{\mathrm{A}}-\mathrm{PS}(1)$ and $\mathrm{NiBh}$ using HeLa (top) and SCCF1 cells (bottom). The graphs display the saturation binding curves of each targeting molecule per cell line. Apparent binding affinity $\left(\mathrm{K}_{\mathrm{D})}\right.$ values of each $\mathrm{NB}$ and conjugate are shown. (b) To perform NB-PDT, a concentration range of $\mathrm{NB}_{\mathrm{A}}-\mathrm{PS}(0.5), \mathrm{NB}_{\mathrm{A}}-\mathrm{PS}(1)$ or $\mathrm{NiBh}$ was incubated with SCCF2 cells for 30 minutes at $37^{\circ} \mathrm{C}$. The graph shows the PS signal on cells detected after washing off unbound conjugate, right before illumination. (c) The viability of the cells was assessed 24 hours after illumination and expressed in percentage relative to non-treated cells.

$\mathrm{NiBh}$, the $\mathrm{NB}_{\mathrm{A}}-\mathrm{PS}$ conjugate with highest payload, was selected as the best agent for EGFR-targeting NB-PDT on feline OSCC cells. The special feature of this conjugate is its high binding affinity to both human and feline cells $\left(\mathrm{K}_{\mathrm{D}} \sim 7-10 \mathrm{nM}\right)$ while having DOC 2.5 (Figure 4a). In addition, the specific binding of NiBh to feline EGFR was maintained, as evidenced by a reduced NiBh binding in the presence of EGF in a concentration dependent manner (Figure 4b). The potency of NiBh as an agent for NB-PDT was evaluated using the panel of human and feline cell lines (Figure 4c). HeLa, SCCF2 and SCCF3 cells were effectively killed after treatment with comparable low nanomolar LD50 values (HeLa, $15.9 \pm 3.7 \mathrm{nM}$; SCCF2, $17.8 \pm 2.2 \mathrm{nM}$; SCCF3, $26 \pm 3.5 \mathrm{nM})$. In line with the very low EGFR expression on MCF7 cells, these cells were only slightly affected at the highest concentrations of the conjugate. On the other hand, the use of $\mathrm{NiBh}$ for NB-PDT on SCCF1 cells resulted in only slight cytotoxicity, contrary to the other cell lines with comparable EGFR expression. To further investigate whether the induced cytotoxicity relies on the specific binding of NiBh to EGFR, NB-PDT was performed in the presence of an excess of unconjugated $\mathrm{NB}_{\mathrm{A}}$. Accordingly, the cytotoxicity decreased under these competing conditions (Figure 4d). Moreover, the NB-PDT effect could be inhibited in the presence of sodium azide $\left(\mathrm{NaN}_{3}\right)$, a singlet oxygen quencher [31] (Figure 4d). To visualize the distinct NB-PDT effect on moderate and low EGFR-expressing cells, SCCF2 and MCF7 cells were stained with fluorescent dyes denoting live/dead cells after NB-PDT. Already early after NB-PDT (2 hours), dead SCCF2 cells were clearly distinguishable and their number increased over time, while only a very small number of dead MCF7 cells could be detected one day after treatment (Figure 4e). 

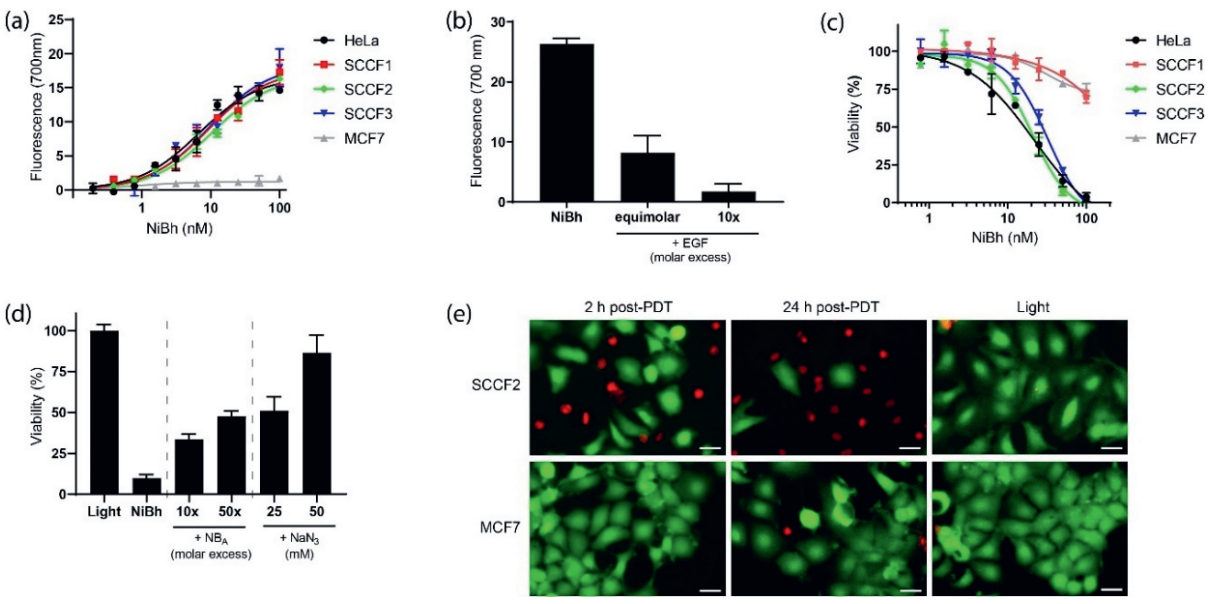

Figure 4 Specific binding and potency of NiBh. (a) A binding assay was performed with $\mathrm{NiBh}$ using the panel of human and feline cell lines (HeLa, SCCF1, SCCF2, SCCF3 and MCF7 cells). The graph displays the saturation binding curves of $\mathrm{NiBh}$ to each cell line from which $\mathrm{K}_{\mathrm{D}}$ values were calculated. (b) Binding of NiBh $(10 \mathrm{nM})$ to SCCF2 cells in the absence or presence of equimolar concentration and 10x molar excess of EGF. The fluorescence corresponding to bound $\mathrm{NiBh}$ under each condition is displayed in the graph. (c) NB-PDT using NiBh was performed on the panel of cell lines and, one day later, viability was assessed. The graph shows viability curves for each cell line, in percentage relative to untreated cells. (d) NB-PDT (50 nM NiBh) was performed on SCCF2 cells in the absence or presence of a molar excess of unconjugated $\mathrm{NB}_{\mathrm{A}}$. NB-PDT was also performed in the presence of sodium azide $\left(\mathrm{NaN}_{3}\right)$. A control consisting of cells exposed to light, but no conjugate, was included. Cell viability was assessed the next day and represented as percentage relative to untreated cells. (e) NB-PDT (50 nM NiBh) was performed on SCCF2 or MCF7 cells and cells stained with calcein and propidium iodide to visualize live (green) and dead (red) cells, respectively. A control in which light was applied, but no conjugate, was taken along. Images were taken 2 and 24 hours after NB-PDT. Scale bar, 20 $\mu \mathrm{m}$.

\section{NB-PDT using NiBh results in the specific killing of feline OSCC cells while leaving surrounding low EGFR-expressing cells unaffected}

To investigate the specificity of NiBh for NB-PDT in a representative and biologically relevant setting, co-cultures were set up consisting of feline OSCC cells (SCCF2), as neoplastic nests, and low EGFR-expressing cells (MCF7) mimicking the surrounding stroma of the oral mucosa. First, NBA-Alexa 647 was employed to visualize its differential binding $\left(4^{\circ} \mathrm{C}\right)$ to both cell lines. As anticipated, NBA was found on the membrane of the feline carcinoma cells, while the detected signal was minimal on the surrounding low EGFR-expressing cells (Figure 5a, top). The next step was to address the accumulation of NBA-Alexa 647 in cells after an incubation time mimicking the incubation with NB-PS conjugate used for the NB-PDT studies $\left(30\right.$ minutes at $37{ }^{\circ} \mathrm{C}$ ). This revealed a predominant accumulation of the NB in the feline tumor cells both membrane-bound and internalized (Figure 5a, bottom). Lastly, NB-PDT using NiBh was performed on the co-cultures and dead cells were visualized via PI staining. Most feline neoplastic cells were killed shortly after treatment ( 2 hours), whereas neighboring low EGFR-expressing cells were left unaffected (Figure 5b). 
(a)
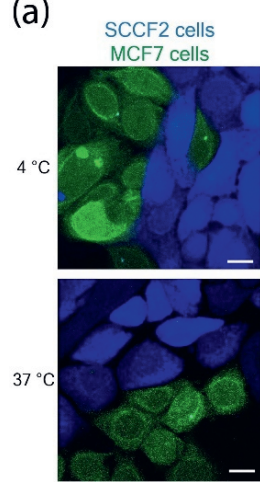
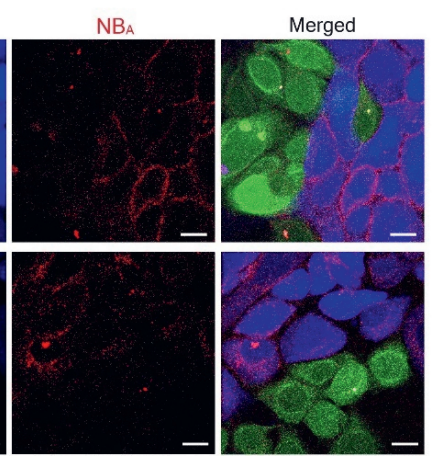

(b)

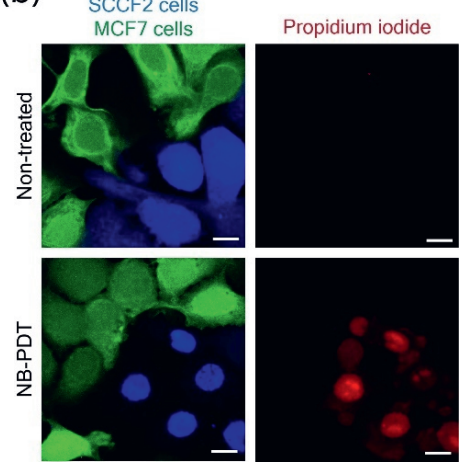

Figure 5 Specificity of $\mathrm{NB}_{\mathrm{A}}$ and $\mathrm{NiBh}$ in co-cultures. SCCF2 cells were labeled with ViaFluor 405 (blue), MCF7 cells with ViaFluor 488 (green), and co-cultured in a 1:1 ratio. (a) Confocal images of cells incubated with $50 \mathrm{nM}$ of $\mathrm{NB}_{\mathrm{A}}$-Alexa 647 (red) for 1 hour at $4{ }^{\circ} \mathrm{C}$ (top) or 30 minutes at $37^{\circ} \mathrm{C}$ (bottom). Scale bar, $15 \mu \mathrm{m}$. (b) Confocal images of cells 2 hours after NB-PDT with $50 \mathrm{nM}$ of NiBh. Cells were stained with propidium iodide to distinguish dead cells (red). Scale bar, $15 \mu \mathrm{m}$.

\section{Discussion}

NB-PDT has emerged as a potent and selective treatment modality for cancer able to effectively kill cancer cells while sparing surrounding healthy tissue. In particular, the use of NB-PDT to treat HNSCC has been demonstrated both in vitro [1] and in the preclinical setting $[2,6]$ with great success. Furthermore, in view of clinical translation, NB-PDT has shown its value using patient-derived HNSCC organoids [8]. This prompted us to advance NB-PDT further and the next logical step was to bring this treatment closer to the human clinic. For this, we first opted to develop NB-PDT for treating companion animals suffering from spontaneous tumors in the veterinary clinic. In particular, we aim to treat cats with OSCC, a tumor type greatly resembling human HNSCC, with no current effective therapeutic options. In the present study, we describe in vitro work that paves the way to the in vivo application of NB-PDT in cats with OSCC. EGFR was selected as a target for feline OSCC and, accordingly, $\mathrm{NB}_{\mathrm{A}}$ was characterized as an EGFR-targeting $\mathrm{NB}$ binding both the human and the feline protein. Next, $\mathrm{NB}_{\mathrm{A}}$ was conjugated to IRDye700DX to yield NiBh, a NB-PS conjugate with high payload (average of 2.5 molecules of PS per NB molecule) which retains high binding affinity to both species. $\mathrm{NiBh}$ was subsequently investigated for NB-PDT to specifically kill feline OSCC cells with EGFR levels comparable to those of neoplastic cells in vivo, whereas neighbouring low EGFR-expressing cells were left unharmed.

Expression of EGFR in feline OSCC has been reported and suggested as therapeutic target [20,21]. Looper et al. found moderate/high EGFR expression in 8 of 13 cases [20], while we found it in 9 out of 10 cases (Figure 1). Despite being a small subset of samples in both instances, it is clear that EGFR is present in feline OSCC to a significant extent. EGFR was confined to the cancer cells arranged in neoplastic nests and absent in the surrounding stroma, which, in the context of NB-PDT, will most likely result in no damage to the structural component of the oral mucosa. Nonetheless, we compared EGFR expression in the adjacent normal oral epithelium and observed that the intensity in the basal layer of this epithelium was similar to the neoplastic cells, in line with the observations in HNSCC $[23,32]$. Intermediate and high EGFR levels have been reported 
in feline normal oral mucosa/tongue [20], but never compared side by side with OSCC tissue. These comparable EGFR levels would render the epithelial cells of the nonlesional mucosa susceptible to EGFR-targeted PDT, but this is not regarded as a concern due to the local nature of PDT and rapid regeneration of the feline oral mucosa [33]. Furthermore, conventional PDT has already been applied in cats for the treatment of SCC and only transient and acceptable local adverse effects were observed with this nontargeted approach [34-37], thus NB-PDT would further minimize these undesired effects.

We consider EGFR the best target with regard to NB-PDT for treatment of feline OSCC. Next to EGFR, only soluble molecules (e.g. VEGF and COX-2) have been reported as highly expressed in feline OSCC [18], which cannot be utilized for NB-PDT. More is known about markers in human HNSCC, where HER2 and HER3 have gained attention. Nevertheless, while EGFR is expressed in more than $90 \%$ of HNSCC and is a clinically validated therapeutic target in HNSCC [38], the other two family members are less commonly expressed [39] and its targeting has resulted in modest clinical success so far [40]. Novel biomarkers for HNSCC that could be used for NB-PDT are emerging, such as CD44, c-Met and PD-L1 [38,41], but further studies are warranted and their presence in feline OSCC is unknown.

It has been described that HNSCC cell lines express higher EGFR levels than what is generally found in the cancer tissue [23]. Therefore, our aim was to ensure that we work with cells expressing clinically relevant EGFR levels, to assess the extent of cytotoxicity induced by NB-PDT that can be expected in the clinic. All feline OSCC cell lines presented membrane EGFR levels comparable to HeLa cells. This is in agreement with our results obtained with patient-derived HNSCC organoids, for which EGFR expression levels were also in the range of HeLa cells [8]. With the idea of clinical relevance in mind, MCF7 cells served as very low EGFR-expressing cells [22], representing the adjacent stroma present around the neoplastic nests in feline OSCC. The first proof of the ability of $\mathrm{NB}_{\mathrm{A}}$ (originally selected against human EGFR) to recognize human and feline EGFR was denoting these differences in EGFR expression between the cell lines, with a comparable trend to a commercial EGFR-targeting, species cross-reactive antibody (Figure 2). This makes $\mathrm{NB}_{\mathrm{A}}$ the first reported NB to bind a feline target, thus joining the small group of NBs developed against targets in companion animals, next to the canine HER2-targeting NBs [42].

In order to use $\mathrm{NB}_{\mathrm{A}}$ for NB-PDT, the critical step of conjugation to the PS was carefully considered. NB-PDT makes use of NB-PS conjugates with good pharmacokinetics for PDT due to the small size of NBs. At the same time, however, this small size allows for only a modest amount of payload (0.5 - 1.5 molecules per NB) without affecting the overall binding affinity of the NB [43]. On the other hand, one antibody can easily carry 3 - 4 drug molecules $[24,44]$. So far, NB-PDT has proven potent using monomeric and dimeric NB-PS conjugates with DOC $0.5-1.5$, using moderate and high expressing cell lines [2,3]. Dimeric NBs (e.g. internalizing biparatopic NBs) facilitate the incorporation of a higher number of PS molecules and have proven to be very potent in vitro [1], but their penetration in a solid tumor is limited by their larger molecular size $[2,45,46]$, and thus not the first choice for the clinic in the context of NBPDT. To ensure a potent NB-PDT effect in a spontaneous tumor and because clinical success has been obtained with antibody-PS conjugates with high DOC [24,25], we developed $\mathrm{NB}_{\mathrm{A}}$ into $\mathrm{NiBh}$, a monomeric NB-PS conjugate with high DOC (2.5). NiBh is, to our knowledge, the first functional monomeric NB with such a high number of payload (e.g. fluorophore or drug) [47].

Importantly, NiBh retained high affinity to both human and feline EGFR, even with high DOC (Figure 3a). This is, so far, a unique feature of $\mathrm{NB}_{\mathrm{A}}$ since other species cross- 
reactive NBs lack this comparable affinity across species $[42,48]$. NiBh could effectively kill the different cell lines with clinically relevant EGFR expression (LD50 in low nanomolar range), while cytotoxicity was not induced with NB-PS conjugates with lower DOC (Figure 3c, 4c). This can be explained by the concept of the threshold dose, which describes that cellular damage is induced only above a certain concentration of reactive oxygen species [49]. The NB-PDT effect induced by NiBh was further characterized as dependent on the binding to EGFR and mediated by the formation of singlet oxygen (Figure 3d), as reported for antibody-targeted PDT employing the same PS [50]. The induced cytotoxicity occurred in an EGFR-dependent manner, a correlation that has been described for NB-PDT with other conjugates $[1,8]$. Only SCCF1 cells did not behave as anticipated, since only a moderate NB-PDT effect was observed, while a more pronounced effect was expected based on the EGFR levels of this cell line. Differences in PDT-induced cytotoxicity have been attributed to variations in the level of antioxidant molecules and enzymes expressed by cancer cells, but this was proved not to be the case for the feline OSCC cell lines (Figure S4). Many other cellular mechanisms play a role in the degree of PDT cytotoxicity [51] and further investigation would be needed to clarify the observed differences in response. Of note, it is clear that the cytotoxicity of EGFRtargeted PDT is independent of EGFR downstream mutations and the presence of membrane EGFR is the main driver. Another important aspect of NB-PDT that differentiates it from conventional PDT is its ability to leave illuminated surrounding normal tissues unharmed. This was indeed the case with $\mathrm{NiBh}$ in co-cultures representing feline OSCC surrounded by low EGFR-expressing stroma cells (Figure 5).

Conventional PDT has already been employed in the veterinary clinic to treat various cancers, such as oral, bladder and prostate carcinomas in dogs and cats [52-55]. Targeted PDT, however, has not yet been applied in companion animals. Thus far, antibody-targeted PDT has shown efficacy in a xenograft mouse model of canine bladder cancer [56], but follow-up studies have not yet been reported. For the treatment of cutaneous, nasal and facial SCC in cats, conventional PDT has yielded variable responses depending on stage and tumor location [34-37]; but, as far as we know, there are no reports concerning treatment of OSCC. We believe that NB-PDT differentiates itself from the efforts made so far with conventional PDT to treat feline SCC. Potential benefits associated to NB-PDT are a more rapid and homogeneous tumor accumulation of the PS, milder local effects due to selective cell killing, increased light penetration through tissues due to the near infrared wavelengths required to activate the PS IRDye700DX, combination of imaging and treatment due to the versatility of IRDye700DX to act as a fluorophore, minimal photosensitivity after treatment, and treatment protocol performed within one day. These are all strong points to support the use of NiBh to treat feline OSCC in the clinic and, consequently, our next efforts will be pointed in this direction. In particular, we will seek to apply NB-PDT as an experimental treatment in client-owned cats with OSCC, having confirmed moderate/high EGFR expression.

In conclusion, $\mathrm{NiBh}$ can be used in vitro to kill feline OSCC cells that express clinically relevant EGFR levels, while sparing surrounding low expressing cells. Therefore, this study presents the use of NiBh for NB-PDT as an attractive therapeutic modality for the treatment of feline OSCC in the veterinary clinic. The species crossreactivity of NiBh and the similarities of feline OSCC with HNSCC make NB-PDT a promising treatment option for human patients and position the translation of NB-PDT to the human clinic one step closer. In the long term, NiBh could be employed against other EGFR-expressing cancers and with a potential use in other species as well. 


\section{Conclusions}

Our results show the development and characterization of a NB-PS conjugate with high payload able to bind both human and feline EGFR, and its use for NB-PDT to kill feline OSCC cells in a specific manner. This paves the way for the application of NB-PDT in the veterinary clinic to treat cats with OSCC. The species cross-reactivity of the conjugate and similarities between feline OSCC and human HNSCC open possibilities to further advance NB-PDT towards the human clinic.

\section{Acknowledgements}

Authors thank Elpetra Timmermans, Adri Slob and Athanasia Papadopoulou for their help and support during the initial phase of this study.

\section{References}

1. Heukers, R.; van Bergen en Henegouwen, P.M.P.; Oliveira, S. Nanobody-photosensitizer conjugates for targeted photodynamic therapy. Nanomedicine Nanotechnology, Biol. Med. 2014, 10, 1441-1451.

2. Van Driel, P.B.A.A.; Boonstra, M.C.; Slooter, M.D.; Heukers, R.; Stammes, M.A.; Snoeks, T.J.A.; De Bruijn, H.S.; Van Diest, P.J.; Vahrmeijer, A.L.; Van Bergen En Henegouwen, P.M.P.; et al. EGFR targeted nanobody-photosensitizer conjugates for photodynamic therapy in a pre-clinical model of head and neck cancer. J. Control. Release 2016, 229, 93-105.

3. Deken, M.M.; Kijanka, M.M.; Beltrán Hernández, I.; Slooter, M.D.; de Bruijn, H.S.; van Diest, P.J.; van Bergen en Henegouwen, P.M.; Lowik, C.W.; Robinson, D.J.; Vahrmeijer, A.L.; et al. Nanobody-targeted photodynamic therapy induces significant tumor regression of trastuzumabresistant HER2-positive breast cancer, after a single treatment session. J. Control. Release 2020, 323, 269-281.

4. De Groof, T.W.M.; Mashayekhi, V.; Shu Fan, T.; Bergkamp, N.D.; Sastre Torano, J.; Van Senten, J.R.; Heukers, R.; Smit, M.J.; Oliveira, S. Nanobody-Targeted Photodynamic Therapy Selectively Kills Viral GPCR-Expressing Glioblastoma Cells. Mol. Pharm. 2019, 16, 3145-3156.

5. Heukers, R.; Mashayekhi, V.; Ramirez-Escudero, M.; de Haard, H.; Verrips, T.C.; van Bergen en Henegouwen, P.M.P.; Oliveira, S. VHH-Photosensitizer Conjugates for Targeted Photodynamic Therapy of Met-Overexpressing Tumor Cells. Antibodies (Basel) 2019, 8, 26.

6. de Bruijn, H.S.; Mashayekhi, V.; Schreurs, T.J.L.; van Driel, P.B.A.A.; Strijkers, G.J.; van Diest, P.J.; Lowik, C.W.G.M.; Seynhaeve, A.L.B.; Hagen, T.L.M. ten; Prompers, J.J.; et al. Acute cellular and vascular responses to photodynamic therapy using EGFR-targeted nanobody-photosensitizer conjugates studied with intravital optical imaging and magnetic resonance imaging. Theranostics 2020, 10, 2436-2452.

7. Beltrán Hernández, I.; Angelier, M.L.; Del Buono D’Ondes, T.; Di Maggio, A.; Yu, Y.; Oliveira, S. The Potential of Nanobody-Targeted Photodynamic Therapy to Trigger Immune Responses. Cancers (Basel) 2020, 12, 978.

8. Driehuis, E.; Spelier, S.; Beltrán Hernández, I.; De Bree, R.; Willems, S.M.; Clevers, H.; Oliveira, S. Patient-Derived Head and Neck Cancer Organoids Recapitulate EGFR Expression Levels of Respective Tissues and Are Responsive to EGFR-Targeted Photodynamic Therapy. J. Clin. Med. 2019, 8, 1880.

9. Oliveira, S.; Heukers, R.; Sornkom, J.; Kok, R.J.; van Bergen En Henegouwen, P.M.P. Targeting tumors with nanobodies for cancer imaging and therapy. J. Control. Release 2013, 172, 607-17.

10. van Straten, D.; Mashayekhi, V.; de Bruijn, H.S.; Oliveira, S.; Robinson, D.J. Oncologic photodynamic therapy: Basic principles, current clinical status and future directions. Cancers 
(Basel). 2017, 9, 1-54.

11. Dolmans, D.E.J.G.J.; Fukumura, D.; Jain, R.K. Photodynamic therapy for cancer. Nat Rev Cancer 2003, 3, 380-387.

12. Beltrán Hernández, I.; Yu, Y.; Ossendorp, F.; Korbelik, M.; Oliveira, S. Preclinical and Clinical Evidence of Immune Responses Triggered in Oncologic Photodynamic Therapy: Clinical Recommendations. J. Clin. Med. 2020, 9, 333.

13. Kobayashi, H.; Choyke, P.L. Near-Infrared Photoimmunotherapy of Cancer. Acc. Chem. Res. 2019, 52, 2332-2339.

14. Stebbins, K.E.; Morse, C.C.; Goldschmidt, M.H. Feline Oral Neoplasia - a 10-Year Survey. Vet. Pathol. 1989, 26, 121-128.

15. van Nimwegen, S.A.; Bakker, R.C.; Kirpensteijn, J.; van Es, R.J.J.; Koole, R.; Lam, M.G.E.H.; Hesselink, J.W.; Nijsen, J.F.W. Intratumoral injection of radioactive holmium ( 166 Ho) microspheres for treatment of oral squamous cell carcinoma in cats. Vet. Comp. Oncol. 2017, 16, 114-124.

16. Bilgic, O.; Duda, L.; Sánchez, M.D.; Lewis, J.R. Feline oral Squamous cell carcinoma: Clinical manifestations and literature review. J. Vet. Dent. 2015, 32, 30-40.

17. Withrow, S. Tumors of the gastrointestinal tract. A. Cancer in the oral cavity. In: Small animal clinical oncology. In Withrow and MacEwen's Small Animal Clinical Oncology; WB Saunders Co: Philadelphia, 2001; pp. 305-316.

18. Wypij, J.M. A naturally occurring feline model of head and neck squamous cell carcinoma. Patholog. Res. Int. 2013, 2013.

19. Feldman, R.; Gatalica, Z.; Knezetic, J.; Reddy, S.; Nathan, C.; Javadi, N.; Teknos, T. Molecular profiling of head and neck squamous cell carcinoma. Head Neck 2016, 38, 1625-1638.

20. Looper, J.S.; Malarkey, D.E.; Ruslander, D.; Proulx, D.; Thrall, D.E. Epidermal growth factor receptor expression in feline oral squamous cell carcinomas. Vet. Comp. Oncol. 2006, 4, 33-41.

21. Bergkvist, G.T.; Argyle, D.J.; Morrison, L.; MacIntyre, N.; Hayes, A.; Yool, D.A. Expression of epidermal growth factor receptor ( EGFR) and Ki67 in feline oral squamous cell carcinomas ( FOSCC ). Vet. Comp. Oncol. 2010, 9, 106-117.

22. Subik, K.; Lee, J.-F.; Baxter, L.; Strzepek, T.; Costello, D.; Crowley, P.; Xing, L.; Hung, M.; Bonfiglio, T.; Hicks, D.G.; et al. The Expression Patterns of ER, PR, HER2 , CK5 / 6 , EGFR , Ki-67 and AR by Immunohistochemical Analysis in Breast Cancer Cell Lines. Breast Cancer Basic Clin. Res. 2010, 4, 35-41.

23. Khaznadar, S.S.; Khan, M.; Schmid, E.; Gebhart, S.; Becker, E.; Krahn, T.; Ahsen, O. Von EGFR overexpression is not common in patients with head and neck cancer. Cell lines are not representative for the clinical situation in this indication. Oncotarget 2018, 9, 28965-28975.

24. Mitsunaga, M.; Ogawa, M.; Kosaka, N.; Rosenblum, L.T.; Choyke, P.L.; Kobayashi, H. Cancer Cell-Selective In Vivo Near Infrared Photoimmunotherapy Targeting Specific Membrane Molecules. Nat. Med. 2011, 17, 1685-1691.

25. Cognetti, D.; Curry, J.M.; Gillenwater, A.M.; William, W.N.; Kochuparambil, S.T.; McDonald, D.; Fidler, M.; Stenson, K.M.; Vasan, N.R.; Razaq, M.A.; et al. A Phase 2a, Multicenter, OpenLabel Study of RM-1929 Photoimmunotherapy in Patients With Recurrent Head And Neck Cancer. Int. J. Radiat. Oncol. 2018, 100, 1368.

26. Tannehill-Gregg, S.; Kergosien, E.; Rosol, T.J. Feline head and neck squamous cell carcinoma cell line: characterization, production of parathyroid hormone-related protein, and regulation by transforming growth factor- $\beta$. Vitr. Cell Dev Biol Anim 2001, 37, 676-683.

27. Martin, C.K.; Dirksen, W.P.; Shu, S.T.; Werbeck, J.L.; Thudi, N.K.; Yamaguchi, M.; Wolfe, T.D.; Heller, K.N.; Rosol, T.J. Characterization of Bone Resorption in Novel In Vitro and In Vivo Models of Oral Squamous Cell Carcinoma. Oral Oncol. 2012, 48, 491-499.

28. Roovers, R.C.; Laeremans, T.; Huang, L.; De Taeye, S.; Verkleij, A.J.; Revets, H.; De Haard, H.J.; van Bergen En Henegouwen, P.M.P. Efficient inhibition of EGFR signalling and of tumour growth by antagonistic anti-EGFR Nanobodies. Cancer Immunol. Immunother. 2007, 56, 303-317.

29. Oliveira, S.; Van Dongen, G. a M.S.; Stigter-Van Walsum, M.; Roovers, R.C.; Stam, J.C.; Mali, 
W.; Van Diest, P.J.; Van Bergen En Henegouwen, P.M.P. Rapid visualization of human tumor xenografts through optical imaging with a near-infrared fluorescent anti-epidermal growth factor receptor nanobody. Mol. Imaging 2012, 11, 33-46.

30. Sabattini, S.; Marconato, L.; Zoff, A.; Morini, M.; Scarpa, F.; Capitani, O.; Bettini, G. Epidermal growth factor receptor expression is predictive of poor prognosis in feline cutaneous squamous cell carcinoma. J. Feline Med. Surg. 2010, 12, 760-768.

31. Sinksb, B.W.P.L.E.; Breitenbacha, T.; Schacka, N.B.; Vinogradovb, S.A.; Ogilby, P.R. Single Cell Responses to Spatially-Controlled Photosensitized Production of Extracellular Singlet Oxygen. Photochem. Photobiol. 2011, 87, 1077-1091.

32. Kriegs, M.; Clauditz, T.S.; Hoffer, K.; Bartels, J.; Buhs, S.; Gerull, H.; Zech, H.B.; Bußmann, L.; Struve, N.; Rieckmann, T.; et al. Analyzing expression and phosphorylation of the EGF receptor in HNSCC. Sci. Rep. 2019, 9, 1-8.

33. Jubb, K.V.F.; Kennedy, P.C.; Palmer, N. Pathology of Domestic Animals. Volume 2; Jubb, K.V.F., Kennedy, P.C., Palmer, N., Eds.; Fourth.; Academic Press, 2012;

34. Bexfield, N.H.; Stell, A.J.; Gear, R.N.; Dobson, J.M. Photodynamic Therapy of Superficial Nasal Planum SquamousCell Carcinomas in Cats: 55 Cases. J Vet Intern Med 2008, 22, 1385-1389.

35. Corrêa, T.P.; Bagnato, V.S.; Ferreira, I.; Rahal, S.C.; Rocha, N.S.; Carvalho, Y.K.; Gouveia, A.H. Hematoporphyrin-based photodynamic therapy for cutaneous squamous cell carcinoma in cats. Vet. Dermatol. 2009, 20, 174-178.

36. Magne, M.L.; Rodriguez, C.O.; Autry, S.A.; Edwards, B.F.; Theon, A.P.; Madewell, B.R. Photodynamic therapy of facial squamous cell carcinoma in cats using a new photosensitizer. Lasers Surg. Med. 1997, 20, 202-209.

37. Friniberger, A.E.; Moore, A.S.; Cincotta, L.; Cotter, S.M.; Foley, J.W. Photodynamic Therapy of Naturally Occurring Tumors in Animals Using a Novel Benzophenothiazine Photosensitizer. Clin. Cancer Res. 1998, 4, 2207-2218.

38. Hsieh, J.C.; Wang, H.-M.; Wu, M.-H.; Chang, K.-P.; Chang, P.-H.; Liao, C.-T.; Liau, C.-T. Review of emerging biomarkers in head and neck squamous cell carcinoma in the era of immunotherapy and targeted therapy. Head Neck 2019, 41, 19-45.

39. Khan, A.J.; King, B.L.; Smith, B.D.; Smith, G.L.; Digiovanna, M.P.; Carter, D.; Haffty, B.G. Characterization of the HER-2 / neu Oncogene by Immunohistochemical and Fluorescence in Situ Hybridization Analysis in Oral and Oropharyngeal Squamous Cell Carcinoma. Clin. Cancer Res. 2002, 8, 540-548.

40. Fayette, J.; Wirth, L.; Oprean, C.; Udrea, A.; Jimeno, A.; Rischin, D.; Nutting, C.; Harari, P.M.; Csoszi, T.; Cernea, D.; et al. Randomized Phase II Study of Cetuximab in Squamous Cell Carcinoma of the Head and Neck ( MEHGAN Study ). Front. Oncol. 2016, 6, 232.

41. Zheng, A.; Li, F.E.N.; Chen, F.; Zuo, J.; Wang, L.E.I.; Wang, Y.; Chen, S.; Xiao, B.; Tao, Z. PD L1 promotes head and neck squamous cell carcinoma cell growth through mTOR signaling. Oncol. Rep. 2019, 41, 2833-2843.

42. Mazzega, E.; De Marco, A. Engineered cross-reacting nanobodies simplify comparative oncology between humans and dogs. Vet. Comp. Oncol. 2018, 16, 202-206.

43. Schumacher, D.; Helma, J.; Schneider, A.F.L.; Leonhardt, H.; Hackenberger, C.P.R. Nanobodies : Chemical Functionalization Strategies and Intracellular Applications. Angew Chem Int Ed Engl 2018, 57, 2314-2333.

44. Sun, X.; Ponte, J.F.; Yoder, N.C.; Laleau, R.; Coccia, J.; Lanieri, L.; Qiu, Q.; Wu, R.; Hong, E.; Bogalhas, M.; et al. Effects of Drug - Antibody Ratio on Pharmacokinetics, Biodistribution, Efficacy, and Tolerability of Antibody - Maytansinoid Conjugates. Bioconjug. Chem. 2017, 28, 1371-1381.

45. Beltrán Hernández, I.; Rompen, R.; Rossin, R.; Xenaki, K.T.; Katrukha, E.A.; Nicolay, K.; van Bergen en Henegouwen, P.; Grüll, H.; Oliveira, S. Imaging of Tumor Spheroids, Dual-Isotope SPECT, and Autoradiographic Analysis to Assess the Tumor Uptake and Distribution of Different Nanobodies. Mol. Imaging Biol. 2019, 21, 1079-1088.

46. Krasniqi, A.; Bialkowska, M.; Xavier, C.; Jeught, K. Van Der; Muyldermans, S.; Devoogdt, N.; 
Huyvetter, M.D. Pharmacokinetics of radiolabeled dimeric sdAbs constructs targeting human CD20. N. Biotechnol. 2018, 45, 69-79.

47. Schumacher, D.; Helma, J.; Schneider, A.F.L.; Leonhardt, H.; Hackenberger, C.P.R. Nanobodies : Chemical Functionalization Strategies and Intracellular Applications Angewandte. 2314-2333.

48. Prantner, A.M.; Yin, C.; Kamat, K.; Sharma, K.; Lowenthal, A.C.; Madrid, P.B.; Scholler, N. Molecular Imaging of Mesothelin-Expressing Ovarian Cancer with a Human and Mouse CrossReactive Nanobody. Mol. Pharm. 2018, 15, 1403-1411.

49. Trachootham, D.; Alexandre, J.; Huang, P. Targeting cancer cells by ROS-mediated mechanisms: A radical therapeutic approach? Nat. Rev. Drug Discov. 2009, 8, 579-591.

50. Peng, W.; Bruijn, H.S. De; Hagen, T.L.M.; Berg, K.; Roodenburg, J.L.N.; van Dam, G.M.; Witjes, M.J.H.; Robinson, D.J. In-vivo optical monitoring of the efficacy of epidermal growth factor receptor targeted photodynamic therapy: The effect of fluence rate. Cancers (Basel) 2020, 12.

51. Agostinis, P.; Berg, K.; Cengel, K..; Foster, T..; Girotti, A..; Gollnick, S..; Hahn, S..; Hamblin, M..; Juzeniene, A.; Kessel, D.; et al. Photodynamic Therapy of cancer: an update. CA Cancer J Clin. 2011, 61, 250-281.

52. McCaw, D.L.; Pope, E.R.; Payne, J.T.; West, M.K.; Tompson, R. V; Tate, D. Treatment of canine oral squamous cell carcinomas with photodynamic therapy. Br. J. Cancer 2000, 82, 1297-1299.

53. Lucroy, M.D.; Ridgway, T.D.; Peavy, G.M.; Krasieva, T.B.; Higbee, R.G.; Campbell, G.A.; Blaik, M.A. Preclinical evaluation of 5-aminolevulinic acid-based photodynamic therapy for canine transitional cell carcinoma. Vet. Comp. Oncol. 2003, 1, 76-85.

54. Xiao, Z.; Owen, R.J.; Liu, W.; Tulip, J.; Brown, K.; Woo, T.; Moore, R.B. Lipophilic photosensitizer administration via the prostate arteries for photodynamic therapy of the canine prostate. Photodiagnosis Photodyn. Ther. 2010, 7, 106-114.

55. L'Eplattenier, H.F.; Klem, B.; Teske, E.; van Sluijs, F.J.; van Nimwegen, S.A.; Kirpensteijn, J. Preliminary results of intraoperative photodynamic therapy with 5-aminolevulinic acid in dogs with prostate carcinoma. Vet. J. 2008, 178, 202-207.

56. Nagaya, T.; Okuyama, S.; Ogata, F.; Maruoka, Y.; Knapp, D.W.; Karagiannis, S.N.; FazekasSinger, J.; Choyke, P.L.; LeBlanc, A.K.; Jensen-Jarolim, E.; et al. Near infrared photoimmunotherapy targeting bladder cancer with a canine anti-epidermal growth factor receptor (EGFR) antibody. Oncotarget 2018, 9, 19026-19038. 



\section{CHAPTER 6}

\section{Preclinical and clinical evidence of immune responses triggered in oncologic photodynamic therapy: clinical recommendations}

Irati Beltrán Hernández ${ }^{1,2, *}$, Yingxin $\mathrm{Yu}^{2,{ }^{*}}$, Ferry Ossendorp ${ }^{3}$, Mladen Korbelik ${ }^{4}$ and Sabrina Oliveira ${ }^{1,2}$

\footnotetext{
1 Pharmaceutics, Department of Pharmaceutical Sciences, Faculty of Science, Utrecht University, Utrecht, the Netherlands

${ }^{2}$ Cell Biology, Neurobiology and Biophysics, Department of Biology, Faculty of Science, Utrecht University, Utrecht, the Netherlands

${ }^{3}$ Department of Immunohematology and Blood Transfusion, Leiden University Medical Center, Leiden, the Netherlands

${ }^{4}$ Department of Integrative Oncology, BC Cancer, Vancouver, Canada

* These authors contributed equally to this work
}

Journal of Clinical Medicine 2020, 9, 333 


\section{Abstract}

Photodynamic therapy (PDT) is an anticancer strategy utilizing light-mediated activation of a photosensitizer (PS) which has accumulated in tumor and/or surrounding vasculature. Upon activation, the PS mediates tumor destruction through the generation of reactive oxygen species and tumor-associated vasculature damage, generally resulting in high tumor cure rates. In addition, a PDT-induced immune response against the tumor has been documented in several studies. However, some contradictory results have been reported as well. With the aim of improving the understanding and awareness of the immunological events triggered by PDT, this review focuses on the immunological effects post-PDT, described in preclinical and clinical studies. The reviewed preclinical evidence indicates that PDT is able to elicit a local inflammatory response in the treated site, which can develop into systemic antitumor immunity, providing long-term tumor growth control. Nevertheless, this aspect of PDT has barely been explored in clinical studies. It is clear that further understanding of these events can impact the design of more potent PDT treatments. Based on the available preclinical knowledge, recommendations are given to guide future clinical research to gain valuable information on the immune response induced by PDT. Such insights directly obtained from cancer patients can only improve the success of PDT treatment, either alone or in combination with immunomodulatory approaches. 


\section{Introduction}

Photodynamic therapy (PDT) is a therapeutic procedure that has proven successful for the treatment of cancer in the clinic [1], as well as non-oncologic indications [2,3]. PDT is approved for the treatment of some skin and organ cancers, such as bladder cancer, squamous cell carcinoma of the head and neck, and esophageal cancer. In addition, numerous clinical trials continue evaluating the use of oncologic PDT [1,4], highlighting the promise of this therapy.

PDT involves the administration of a light-activatable molecule or photosensitizer (PS), which accumulates at the tumor area, driven by the enhanced permeability and retention effect (EPR), and subsequent local illumination of the tumor tissue to excite the PS. This triggers photochemical reactions that generate numerous highly reactive oxygen species (ROS), eventually leading to direct cytotoxic effects on the tumor cells. Of note, PDT protocols involving a short time interval between PS administration and light application mainly target the tumor vasculature and, in this context, the tumor cell death is largely attributed to vascular occlusion, an approach known as vascular PDT [5]. In either case, damage to surrounding normal tissues is limited and this strategy is thus considered to be selective to the treated tumor site [6], especially when compared with other conventional anticancer strategies.

Preclinical studies describe three different but interrelated mechanisms that contribute to the tumor growth control and occasional complete tumor destruction observed after PDT treatment [7,8] (Figure 1). First, PDT-generated ROS directly kill malignant cells at the primary site, via mainly apoptosis, necrosis and/or autophagy. Secondly, PDT damages the endothelial cells of tumor-associated vasculature, resulting in a significant decrease in blood flow that can lead to tumor death due to starvation. These two mechanisms are responsible for the initial tumor ablation, which appears to trigger an early localized inflammatory response that constitutes the third antitumor mechanism. This early response can activate the immune system and thereby facilitate the clearance of remaining tumor cells in the treated site. In a later phase, an adaptive immune memory may develop as well, leading to a systemic response, capable of preventing tumor recurrence and the formation of tumor metastases, in the long term. Immunostimulation in this context appears to be dependent on the two other antitumor mechanisms, i.e., the type of tumor cell death mediated by ROS and the extent of the tumor-associated vasculature damage.

Tumor cells benefit from a dysfunctional immune environment incapable of eliciting an antitumor response partly due to the absence of factors that can stimulate innate immune cells [9]. PDT seems to overcome this dysfunction by induction of immunogenic tumor cell death pathways, mainly immunogenic apoptosis and necrosis. An innate immune response can be triggered by the exposure or release of danger signals from dying and damaged cells, so-called damage-associated molecular patterns (DAMPs) $[9,10]$. These DAMPS alone or in association with tumor antigens can be recognized by antigen presenting cells (APCs), which may result in the development of an adaptive immune response against the tumor [10-12] (Figure 1).

PDT is now established as a clinical treatment for some cancers and non-malignant diseases, but it is still underutilized in the clinic and it has not yet reached its full potential. Although antitumor immunity after PDT has been reported in animal tumor models, the role of the immune system in the therapeutic outcome of clinical PDT is still unclear. Of note, studies use a wide range of PDT protocols that include different PS, doses, tumor models, and illumination conditions. All these factors can affect the development of 
antitumor immunity and treatment outcome, making it challenging to compare studies side by side and draw solid conclusions.

In this review, substantial evidence of the immunostimulatory effects of PDT is provided. For this, we begin by describing the possible mechanism of how PDT induces immune responses, followed by the evidence of immune responses post-PDT, from preclinical studies (particularly using the PS aminolevulinic acid (ALA) or Photofrin), to the available clinical studies reporting on these effects. Special attention is also given to combined strategies described at the preclinical level to exploit the immunobiology of PDT for more potent and prolonged responses. Lastly, recommendations are given for future clinical trials to collect additional information on the evidence of immune responses, and critical points for the translation of the available preclinical knowledge into the clinic are also discussed here. Importantly, when the recommendations given are implemented in new clinical trials, significant and unique information will be obtained, which is expected to contribute to a better understanding of PDT effects and to improve the success of treatment.

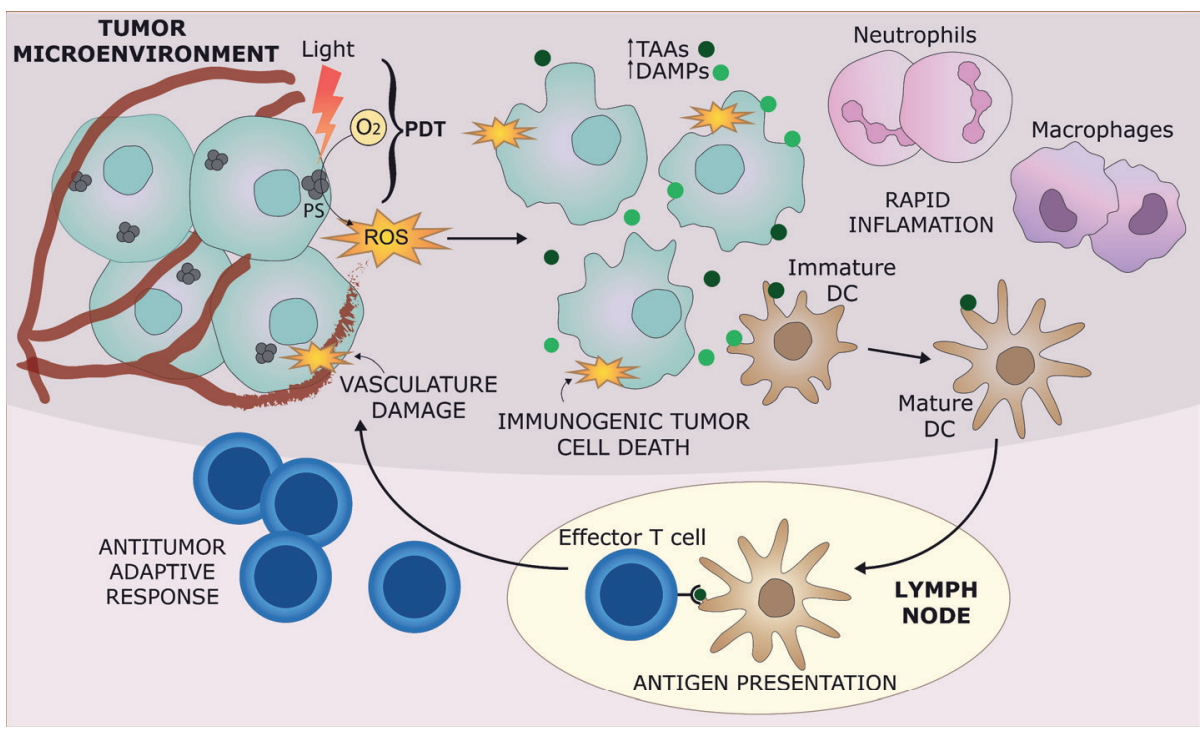

Figure 1 Overview of the antitumor mechanisms of PDT. PDT combines light, oxygen, and a photosensitizer (PS) resulting in the generation of reactive oxygen species (ROS) within tumor cells and tumor-associated vasculature. This leads to a direct cytotoxic effect in tumor cells as well as vasculature shutdown, which in turn results in tumor death due to starvation. This initial tumor destruction triggers a rapid localized inflammation at the tumor site consisting mostly of neutrophils and macrophages. ROS induce an immunogenic tumor cell death (mainly apoptosis and necrosis) that involves exposure/release of damage-associated molecular patterns (DAMPs) from dying cells. Antigen presenting cells (mainly dendritic cells) will be stimulated by these DAMPs, engulf tumor associated antigens (TAAs), and present antigenic peptides to effector T cells, thereby orchestrating an antitumor adaptive response, which could provide systemic tumor immune control in the long term.

\section{How PDT Induces Immune Responses against the Treated Tumor}

The critical event for the development of PDT-induced antitumor response is the engagement of cellular stress signaling networks caused by the infliction of oxidative 
stress in targeted cancer cells, that creates a threat of proteostasis impairment [13]. These evolutionarily well-preserved canonic protection mechanisms are based on the elaborate and universal stress response signaling mediated by a network of harmonized signal transduction pathways [14]. The means for controlling tissue homeostasis at the PDTtreated site includes molecular repair mechanisms for recovery of stressed cells, local inflammatory responses, elimination of impaired tissue components and of damaged cells. The latter encompasses not only programmed cell death mechanisms (apoptosis, necroptosis, pyroptosis, autophagy, and others), but also immune rejection [13].

Stress signal transduction pathways are usually triggered in cells of PDT-treated tumors by sensors (kinases or transcription elements) released from repressed state by the presence of stressors, mostly proteins becoming unfolded/misfolded due to the photooxidative damage. Such sensors reportedly involved in response to PDT include inositol-requiring element-1 (IRE1), activating factor 6 (ATF6), protein kinase R-like ER kinase (PERK), heme regulator inhibitor kinase (HRI) from unfolded protein response (UPR), and integrated stress response (ISR) pathways [13]. These sensor kinases phosphorylate, upon activation, their downstream responders, which are nuclear transcription factors that can now translocate from cell cytoplasm into nucleus and bind to stress response elements located in the promoters of targeted genes [15]. This leads to the expression of certain genes encoding stress-relieving proteins dedicated to the maintenance of proteostasis, which range from chaperons, other proteins that become DAMPs, antioxidant proteins, to factors promoting cell death [13]. Stress signaling mediated by PERK and IRE1 controls the activities of NF- $\kappa B$ and Toll-like receptors (TLRs) that are recognized as critically important in PDT-induced host responses $[11,16,17]$. Various stress signaling pathways interact at multiple points with signals regulating innate and adaptive immune activity and immunogenic cell death development, as well as controlling the activity of regulatory immune cells $[13,17]$. Another important circumstance is that stressed cells become highly immunogenic [18]. This is mainly due to cryptic translation events triggered by stress signaling-mediated accrual of alternate initiation factors capable of translating normally untranslated RNA regions [19], which results in the expression of neoantigens that are not controlled by immunotolerance mechanisms. In such a way, PDT can elicit a strong immune response against treated tumors, which appears to share features with other rapid tumor ablating modalities induced cellular stress response, including those based on thermal effects, high hydrostatic pressure treatment, and electric effect $[20,21]$.

\section{Preclinical Evidence of Antitumor Immunity Induced by PDT}

Here, we first describe the effect of PDT on the innate immunity, followed by that on adaptive immunity, particularly focusing on the PS Photofrin and ALA. This choice of PSs is based on the fact that long-established PDT protocols are used for these PSs, hereby minimizing the influence that the PDT protocol can have on the subsequent immunomodulation. Furthermore, both PSs are two of the few extensively investigated in animal studies and the ones mostly investigated in cancer patients (even if in few studies in total), thus easily enabling correlation of data and translation of knowledge into the clinic. A summary of the main characteristics and aspects concerning Photofrin and ALA are presented in Table 1. 
Table 1 Principal characteristics of the photosensitizers Photofrin and ALA

\begin{tabular}{|c|c|c|}
\hline Photosensitizer (PS) & Porfimer Sodium & ALA \\
\hline Trade name & Photofrin & Levulan \\
\hline Composition & $\begin{array}{c}\text { Mixture of hematoporphyrin } \\
\text { derivatives }\end{array}$ & $\begin{array}{c}\text { Heme precursor (prodrug) } \\
\text { converted to Protoporphyrin IX }\end{array}$ \\
\hline Maximum absorption & $630 \mathrm{~nm}$ & $630-635 \mathrm{~nm}$ \\
\hline $\begin{array}{c}\text { Adsorption at } \\
\text { maximum wavelength }\end{array}$ & $3000 \mathrm{M}^{-1} \mathrm{~cm}^{-1}$ (weak) & $5000 \mathrm{M}^{-1} \mathrm{~cm}^{-1}$ (weak) \\
\hline Administration & Systemic (i.v.) & Systemic (i.v.), oral, topical \\
\hline $\begin{array}{l}\text { Time of illumination } \\
\text { after PS administration }\end{array}$ & $40-50 \mathrm{~h}$ & Within $24 \mathrm{~h}$ \\
\hline Clinical dose & $1 \mathrm{mg} / \mathrm{kg}$ & $10-20 \%$ ALA emulsion (topical) \\
\hline Illumination conditions & $\begin{array}{l}80-260 \mathrm{~J} / \mathrm{cm}^{2} \\
\leq 150 \mathrm{~mW} / \mathrm{cm}^{2}\end{array}$ & $\begin{array}{r}75-260 \mathrm{~J} / \mathrm{cm}^{2} \\
\leq 150 \mathrm{~mW} / \mathrm{cm}^{2} \\
\end{array}$ \\
\hline $\begin{array}{l}\text { Singlet oxygen } \\
\text { quantum yield }\end{array}$ & 0.89 (high) & 0.56 (moderate) \\
\hline PS localization & Mitochondria & $\begin{array}{l}\text { Cell membrane, mitochondria, } \\
\text { lysosome }\end{array}$ \\
\hline Induced cell death & Apoptosis (mainly) $*$ & Apoptosis (mainly) $*$ \\
\hline Disadvantages & $\begin{array}{l}\text { Limited tissue penetration } \\
\text { Skin photosensitization (6 } \\
\text { weeks) }\end{array}$ & $\begin{array}{l}\text { Limited tissue penetration } \\
\text { Moderate pain if skin treatment }\end{array}$ \\
\hline Approved indications & $\begin{array}{l}\text { Bladder, esophageal, skin, and } \\
\text { non-small cell lung and cancer }\end{array}$ & $\begin{array}{l}\text { Actinic keratosis and other non- } \\
\text { oncologic indications }\end{array}$ \\
\hline Ongoing clinical trials & $\begin{array}{c}\text { Brain, cervical, breast and } \\
\text { head and neck cancer, among } \\
\text { others }\end{array}$ & $\begin{array}{l}\text { Basal cell carcinoma, cervical } \\
\text { neoplasia, and head and neck } \\
\text { cancer, among others }\end{array}$ \\
\hline
\end{tabular}

* Apoptosis is the main mechanism reported in vitro, although necrosis is often described in vivo.

\section{PDT and the Innate Immune System}

\section{Neutrophils}

Being the most numerous leukocytes in human body, neutrophils are capable of causing damage at inflammatory sites, and influencing other immune cell functions. Rapid and massive increase of neutrophils is one of the manifestations in PDT-mediated acute inflammation. However, this notion is mainly derived from studies using Photofrin. Within $5 \mathrm{~min}$, high numbers of neutrophils can already be seen at the treated tumor site [22], and this lasts until $24 \mathrm{~h}$ after PDT. More recently, a noninvasive manner has been developed to monitor neutrophil activation at the tumor site using in vivo imaging of luminol chemiluminescence [23]. Of note, pronounced neutrophilia in blood was also observed in mice treated with Photofrin-PDT, which is composed of an early wave of elevation in blood neutrophil levels during the initial hours $(3-4 \mathrm{~h})$, followed by an even more pronounced increase around 8-10 h after PDT [24-26]. Such surge is presumably an accelerated generation and mobilization of neutrophils from the bone marrow [26], promoted by IL-1 and Granulocyte colony-stimulating factor (G-CSF) that are known to 
be induced by PDT [27]. In ALA-PDT, these two waves of neutrophilia have not been characterized. A dramatic increase of neutrophils in blood and at the tumor site in rats has been described after systemic ALA-PDT [28].

The infiltration of neutrophils into the tumor sites is thought to be mediated by the intercellular adhesion molecule of ICAM-1 [29,30] and L-selectin [25,31] expressed on the tumor vascular endothelium, as well as ICAM-1 ligands (CD11b/CD18 and $\mathrm{CD} 11 \mathrm{c} / \mathrm{CD} 18)$ that are upregulated on neutrophils following Photofrin-PDT [30]. Although KC (mouse analog of human CXCL1) and CXCL2 are reported as major chemoattractants for the tumor infiltration of neutrophils after HPPH (2-[1hexyloxyethyl]-2-devinyl pyropheophorbide-a)-PDT [32], these molecules have not been described in Photofrin-PDT. Besides, vasodilatation and increased vascular permeability were observed after ALA-PDT [33], which may contribute to the increased infiltration of immune cells into the tumor.

Generation of neutrophil responses has been suggested to be particularly important in the case of Photofrin-PDT. This has been reported using immunocompetent and neutrophemic rats, where the success of Photofrin-PDT depends on the number of circulating neutrophils [34]. In addition, in a recent study using a mouse model, stronger activation of neutrophils at the tumor site was shown to be prognostic for a complete response after Photofrin-PDT in the long term. However, the role of neutrophils in ALAPDT seems less crucial than in Photofrin-PDT. Using neutrophemic rats, it has been demonstrated that depletion of neutrophils did not significantly change the cure rates of ALA-PDT [28]. It is important to note that these two studies had the same tumor animal model, PS administration route, and time interval between PS administration and light application $[28,34]$; however, they differ in size of the treated tumors, induction method of neutropenia, and illumination regimens, which may, at least partly, explain this discrepancy.

\section{Complement}

Since no characterization of the complement response in animals has yet been provided in the case of ALA-PDT, this section mainly discusses studies using Photofrin. The complement system is involved in the initial recognition of tumor damage, recruitment of immune cells to the tumor site, removal of damaged/dead cells, and direct tumor lysis [35]. In fact, systemic and local complement activation has been observed following Photofrin-PDT treatment of solid tumors. Complement protein C3 has been identified as an essential chemoattractant for the advanced inflammatory infiltration $[25,35]$ and, to a much lesser extent, for the early phase as well [25]. Blockage of C3a receptor significantly inhibits the advanced phase of neutrophilia (which appears $8 \mathrm{~h}$ after PDT) [24], illustrating the role of $\mathrm{C} 3$ in propagating the advanced neutrophilia. The rise of C3 protein in serum, however, occurs at the post-PDT time period when the neutrophilia is largely resolved [26]. Actually, C3 serum levels even temporarily drop shortly after PDT, but reach their peak at 24-72 h, and remain elevated within the first week [26,36]. The initial decline can, most likely, be explained by the elevated consumption of $\mathrm{C} 3$. Indeed, the activation of complement, as indicated by erythrocyte hemolysis, correlates with the time kinetics of PDT-induced neutrophilia. In addition, upregulation of C3a receptor on peripheral neutrophils and monocytes was observed at $8 \mathrm{~h}$ after PDT. The complement system can be activated by antibody complexes, so called classical pathway, or by damaged tissue and altered cell surfaces via the alternative pathway [35]. PDT-induced complement activation has been suggested to occur via the alternative pathway, since neutrophilia is also observed in severe combined immunodeficient (SCID) mice after PDT, which are deficient in T and B cells. 
The membrane attack complex (MAC), assembled by the complement proteins C5b-9, has been observed on the cell membrane of both tumor and endothelial cells as early as $30 \mathrm{~min}$ post-PDT [29]. Interestingly, this lytic pore can also act as leukocyte chemoattractant. Furthermore, tumor cells after PDT are more vulnerable to complement attack, partly because of the downregulated expression of membrane-bound complement regulatory proteins (mCRPs), which prevent complement attack, on the surface of PDTtreated tumor cells [37].

\section{Immunogenic Cell Death}

Physiological apoptosis is regarded as a tolerogenic mode of cell death in immunological terms. More recently, two morphologically equivalent but immunologically distinct subclasses of apoptosis, i.e., immunogenic and non-immunogenic apoptosis, were described giving rise to the new concept of immunogenic cell death (ICD) [38,39]. It is a form of cell death eliciting activation of the immune response in a manner similar to pathogen-infected cells [40]. ICD is characterized by exposure or release of DAMPs, such as calreticulin (CRT), high mobility group box 1 (HMGB1), heat shock protein (HSP)70/90, and ATP. Through binding to pattern-recognition receptors (PRRs), e.g., TLRs, these DAMPs elicit activation of immune responses as endogenous analogs to pathogenassociated molecular patterns (PAMPs). For instance, ICD induces a chemokine release profile capable of recruiting neutrophils, which can eliminate residual viable cells, in a similar fashion as upon pathogenic incursion [40]. ICD is not unique to apoptosis [41]. Cell death through necrosis, necroptosis, pyroptosis, or autophagy can also induce immunogenicity. It has been shown that ICD depends on the generation of ROS and the phosphorylation of eukaryotic initiation factor $\alpha$ (eIF2 $\alpha$ ), which is one of the three arms of the ER stress response [41,42]. PDT can induce large amount of ROS production inside the cancer cells, thereby causing oxidative stress-based cell death. In other words, PDT may turn "cold" tumors into "hot" tumors by inducing ICD. Being known as a strong inducer of ROS-based ER stress, Hypercin-PDT is the first PDT identified as being an ICD inducer [43]. In fact, the capacity of Photofrin- or ALA-PDT to induce tumor ICD has also been explored. In particular, Photofrin-PDT treated tumors exhibit a marked increase of the surface HSP70, as well as a slight increase of chaperone GPR78, at $16 \mathrm{~h}$ post-PDT [11]. In another study, Korbelik et al. showed that, shortly after Photofrin-PDT (at $1 \mathrm{~h}$ ), cell surface expression of CRT already increases on tumors, and HMGB1 rises in serum [44]. Application of topical ALA-PDT increases expression of several other DAMPs (e.g., CRT, HSP70, and HMGB1) in tumors within the first $9 \mathrm{~h}$, leading to subsequent maturation of dendritic cells [45].

It is important to note that ICD is a prerequisite for induction of effective immune responses against cancer [46]. A "gold standard" protocol for the in vivo assessment of ICD in mice relies on vaccination with treated cancer cells, followed by re-challenge with living cancer of the same type in syngeneic immunocompetent animals [47]. Gollnick et al. were the first to demonstrate that Photofrin-PDT-generated tumor cell lysates are immunogenic and can be used as an anticancer vaccine. Moreover, these PDT-based cancer vaccines are significantly more protective than other ways of generating cancer vaccines (UV, freeze/thaw, or ionizing irradiation) [48]. Vaccination with Photofrin-PDT treated tumor cells induces DC maturation, increases IFN- $\gamma$ and TNF- $\alpha$ secretion by splenocytes, as well as their cytolytic activity $[48,49]$, and is effective against poorly immunogenic tumors [50]. The systemic tumor-specific immune response has also been reported in the case of ALA [45], where a complete and strong tumor protection is seen in challenged mice. Likewise, vaccination with DCs stimulated by ALA-PDT treated tumor cells is more potent and effective than the one obtained with DCs co-cultured with 
tumor cells treated with freeze/thaw [12]. Together, these data provide evidence for the capacity of PDT being a strong ICD inducer and a promising strategy for the use of PDT in the generation of cancer vaccines.

\section{Dendritic Cells}

Being a major subpopulation of antigen presenting cells, dendritic cells (DC) phagocytize damaged tumor cells and process their antigens and migrate to local lymph nodes, where in optimal environment they could present antigens to naïve $\mathrm{T}$ cells, leading to subsequent $\mathrm{T}$ cell proliferation and activation. PDT treatment influences this process in many ways. For instance, efficient phagocytosis of dead tumor cells by DC after Photofrin-PDT [10] increased number of IL-12 expressing CD11c+ DCs in the tumor draining lymph nodes at $24 \mathrm{~h}$ after Photofrin-PDT [51], and their enhanced capacity to stimulate $\mathrm{T}$ cell proliferation and IFN-y release [51]. Similarly, accumulation of DCs was also observed at the tumor site at $24 \mathrm{~h}$ after ALA-PDT [52]. Of note, immature DC are poor T-cell stimulators, which rather tend to induce specific immune tolerance [53]. Hence, DC maturation is a critical step in the induction of the immune response. In fact, accumulating evidence from Photofrin- or ALA-PDT supports that PDT-treated tumor lysates enhance DC maturation in vitro, as indicated by their enhanced expression of surface CD86, CD80, and MHCII, as well as IL-12 release $[45,48,54]$. This is most likely induced by the accumulation of DAMPs at the tumor site, yet further studies are needed to elucidate the exact mechanism.

In addition, other innate components such as mast cells and macrophages have been addressed in the PDT-induced innate immune response, although to a lesser extent. Increased numbers of these cells were seen at the tumor site [22] or peritoneally [24] within $24 \mathrm{~h}$ after Photofrin-PDT. Remarkably, it appears that NK cells contribute largely to the cure rates of tumor-bearing SCID mice after Photofrin-PDT [55], but such effect was not observed in the immunocompetent host. In conclusion, preclinical studies have provided consistent and detailed insights into the innate immune responses induced by Photofrin-PDT, such as neutrophilia, complement activation, exposure of DAMPs and increased DC activity (a number of these studies have been summarized in Table 2). Characterization of these responses is however less defined in ALA-PDT that is often applied topically for treating superficial cancer. Distinct microcirculatory effects have been described between systemic and topical application of ALA [33]. However, it is unknown if the application route could influence the host immune responses, since data were not provided in these studies [33,52]. 


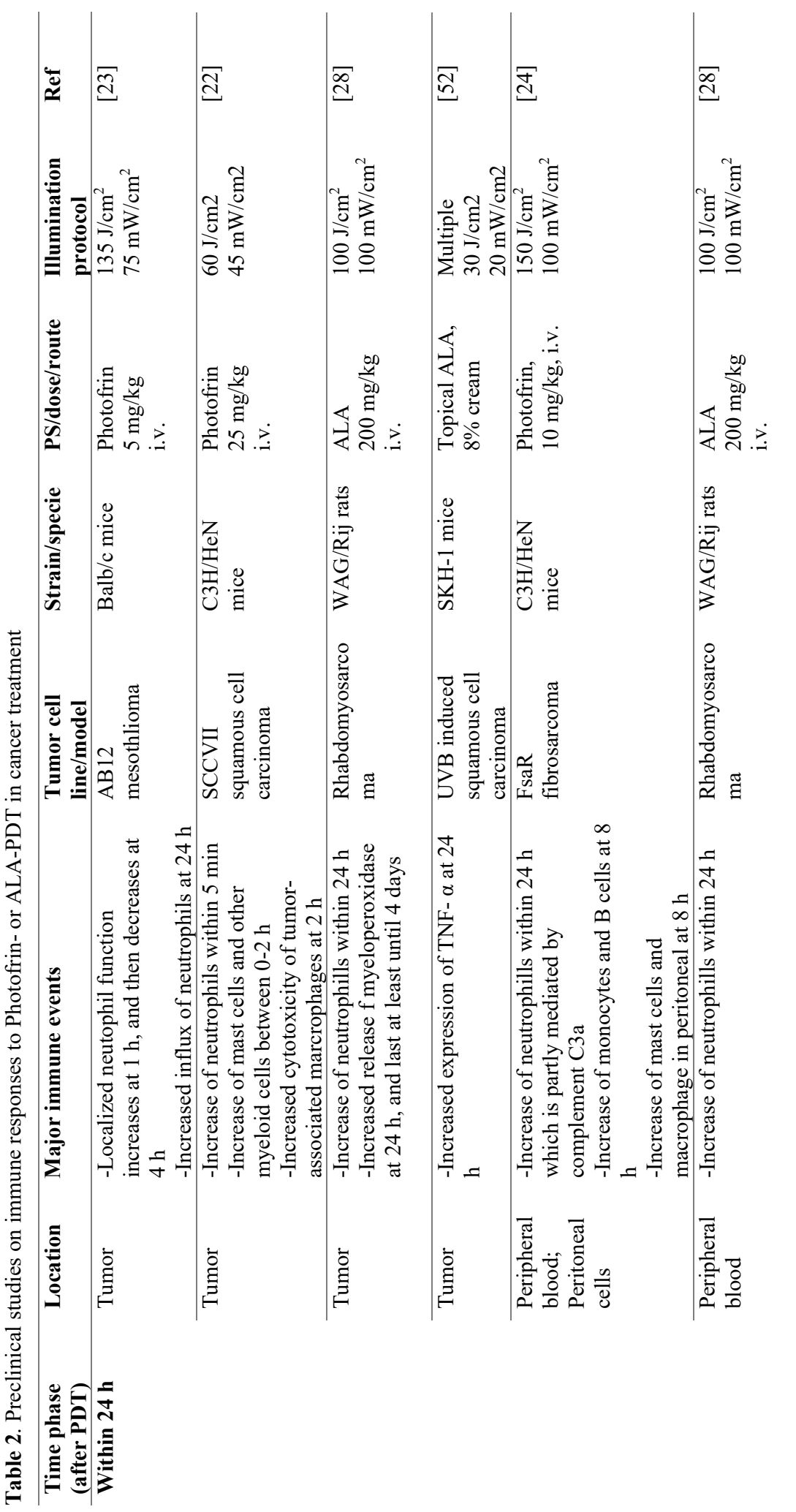




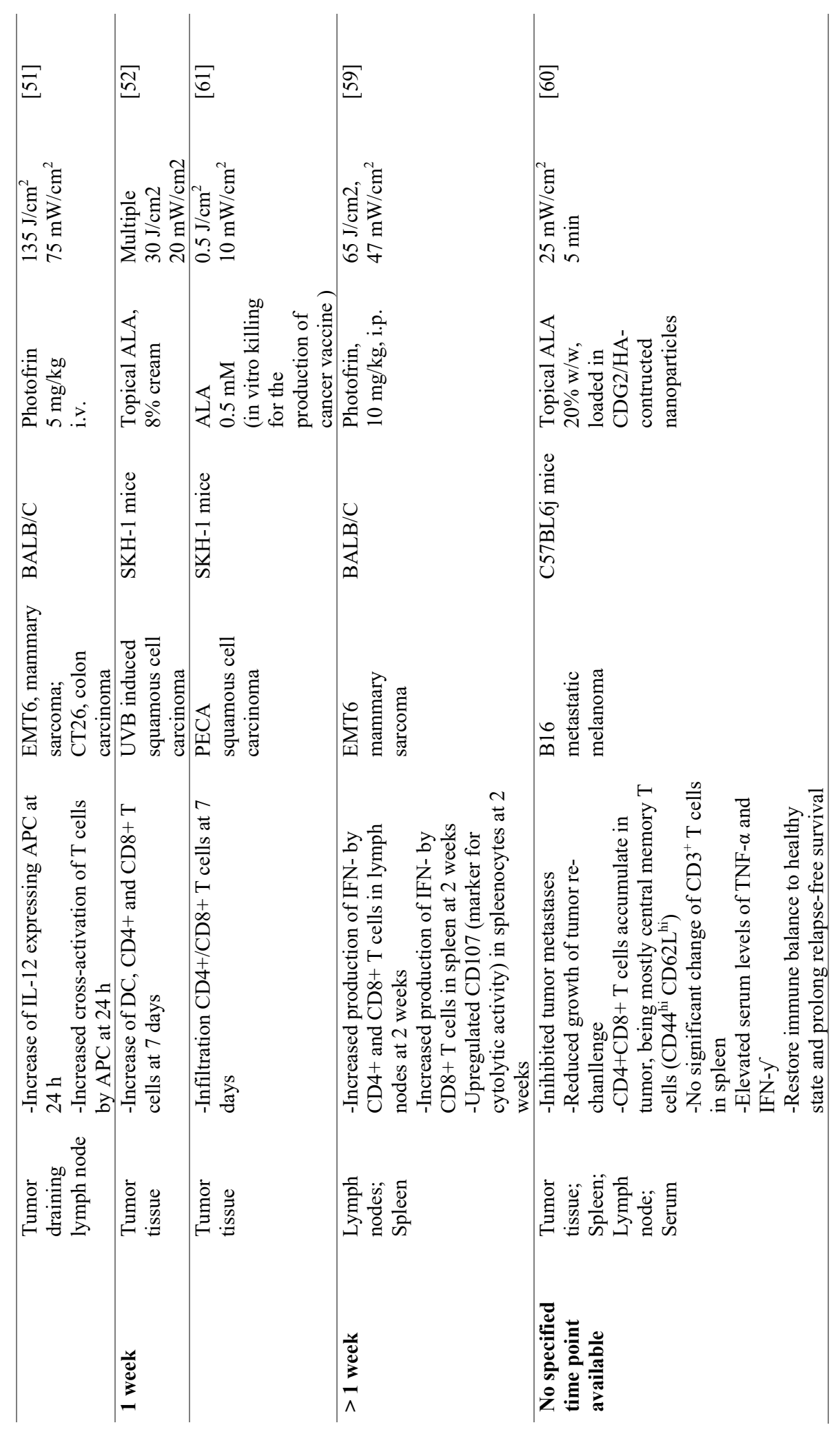




\section{PDT and the Adaptive Immune System}

The key role of adaptive immunity in PDT-induced antitumor immunity was first addressed by Korbelik et al., who described that Photofrin-PDT can initiate tumor cell killing in both immunocompetent and immunodeficient mice (SCID or nude mice), whereas no long-term cure of tumors was observed in the latter [56]. The same group further demonstrated that adoptive transfer of splenocytes from the immunocompetent mice cured by PDT into SCID mice fully restored the curative effect of PDT. These adoptive splenocytes are antigen-specific, since the tumor rejection occurs only when mice were inoculated with tumors of the same origin [55]. Interestingly, such adoptive transfer obtained from tumor-bearing mice cured using X-rays were much less effective [55], suggesting a stronger immunogenic impact of tumor cell death triggered by PDT.

Evidence points to CD8+ T lymphocytes as the main player in PDT-mediated antitumor immune response. This is manifested by the significantly reduced or even abrogated curative effect of PDT upon selective CD8+ T cell depletion [55,57]. In some cases, the induced antitumor immune response is systemic, and therefore it can also be potent outside the primary tumor area. Kabingu et al. [58] described regression of distant lung tumors after local treatment of subcutaneous tumors with Photofrin-PDT. Such systemic control of tumors was accompanied by an increased cytotoxicity of splenocytes against the tumor and infiltration of CD8+ T lymphocytes in the untreated tumor. CD8+ depletion abolished the systemic effect of PDT to control distant tumors outside the primary tumor area. Interestingly, the effect of CD8+ cells appears to be dependent on the presence of NK cells, rather than CD4+ T lymphocytes [58]. In addition, a significant increase of memory $\mathrm{T}$ lymphocytes (CD44hiCD45RBlow) was detected in the lymph nodes of mice treated with Photofrin-PDT [31], indicating the development of immune memory. Wachowska et al. showed an increased production of IFN- $\gamma$ in both CD4+ and CD8+ T lymphocytes isolated from the lymph nodes, as well as splenic CD8+ T lymphocytes, of mice treated with Photofrin-PDT. Moreover, these CD8+ T lymphocytes appear to be highly cytolytic, as indicated by their increased expression of the cytolytic marker CD107 [59]. In contrast, not much information about the ALA-PDT induced adaptive immunity is available. A study has shown that memory $\mathrm{T}$ cells (CD44hiCD62Lhi) accumulate in the tumor and spleen of ALA-PDT treated mice [60].

In conclusion, the systemic and long-term immune effect of PDT relies largely on adaptive immunity, and data have shed light on the role of $\mathrm{T}$ lymphocytes, with a focus on CD8+ $\mathrm{T}$ cells (as exemplified in Table 2). The importance of humoral adaptive immunity has, however, not been thoroughly demonstrated, although a study has shown that expression of $\mathrm{C} 3 \mathrm{a}$ receptor increased on B lymphocytes after Photofrin-PDT, while its expression remained unchanged on T lymphocytes [24]. Since CD8+ T lymphocytes play an essential role in antitumor responses, the mechanism of how PDT induces activation of these cells would therefore be of prime interest in future research.

\section{Clinical Evidence of Antitumor Immunity Induced by PDT}

Although Photofrin and ALA have been used in numerous clinical trials, only a small number of these have investigated the immune responses of PDT. Most clinical data derive from studies applying topical ALA-PDT to patients with basal cell carcinoma (BCC), while fewer studies investigate this aspect of PDT in the case of MAL (the methyl ester of ALA), Photofrin, or Temoporfin (Table 3). 


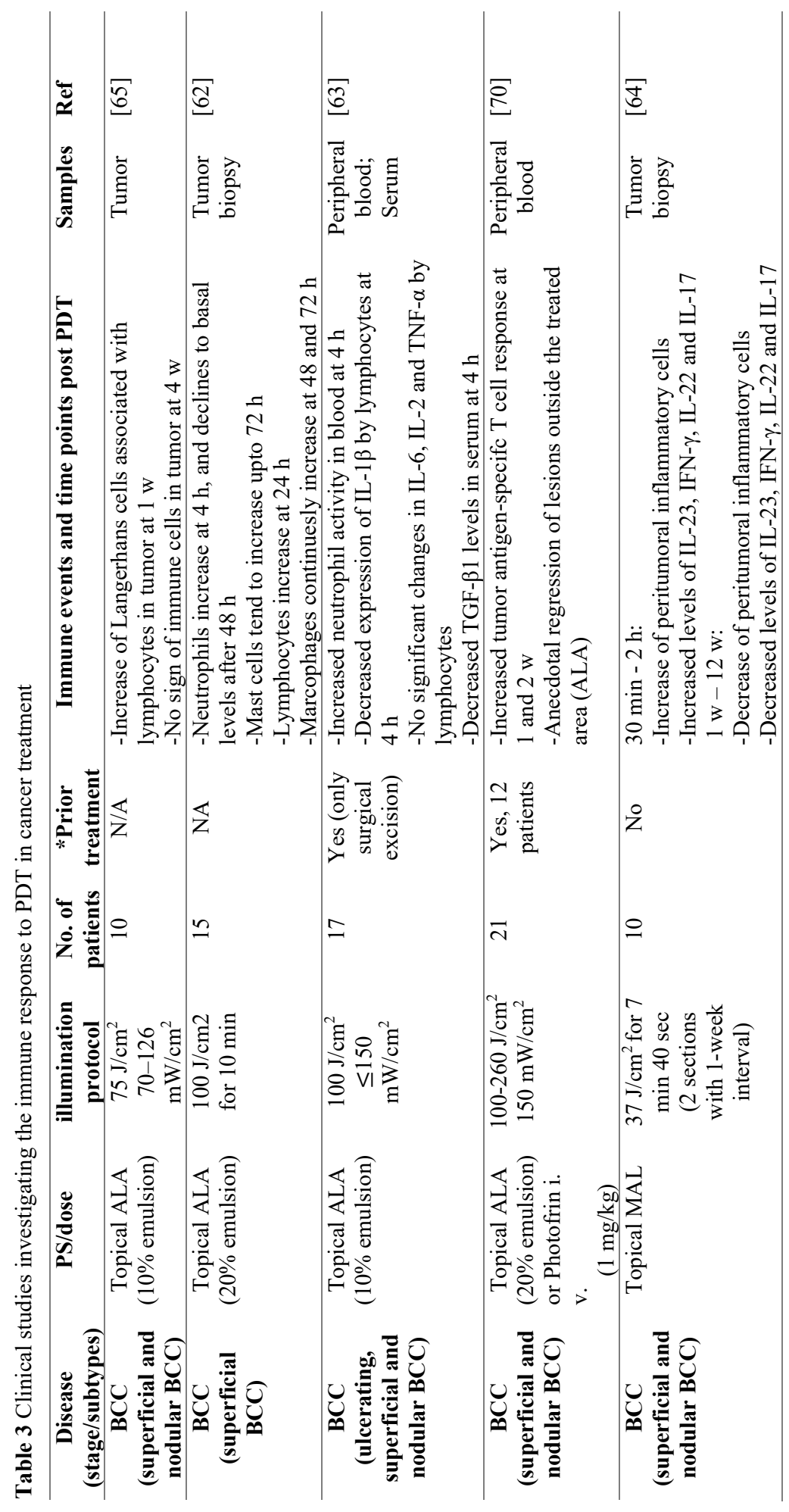




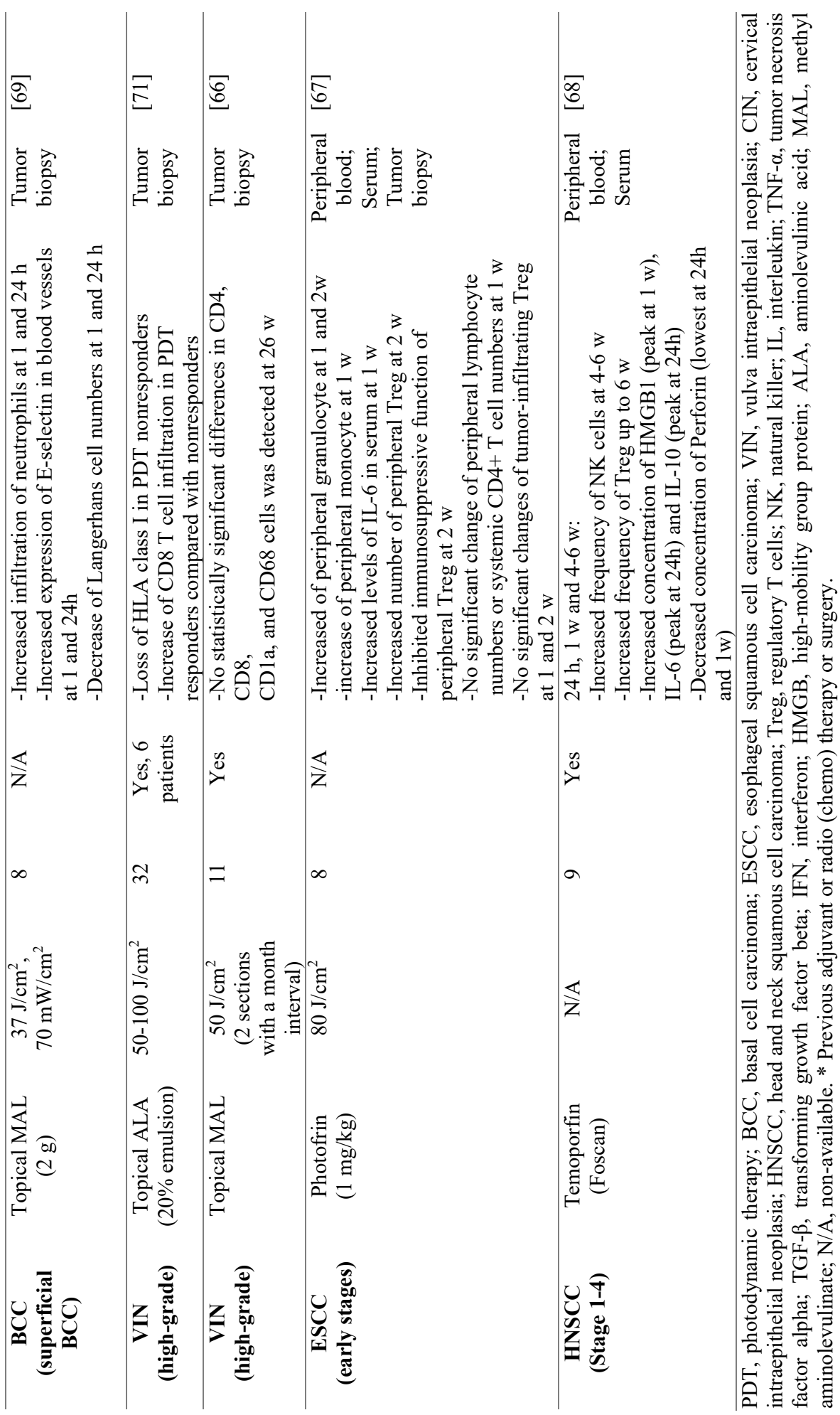




\section{Acute Immune Response}

Acute immune responses are confirmed in BCC patients treated with topical ALA- or MAL-PDT. Within $1 \mathrm{~h}$ after PDT, increased infiltration of neutrophils is already visible at the tumor site, reaching its peak at $4 \mathrm{~h}$, and declining to basal level after $48 \mathrm{~h}$ [62]. Interestingly, activity of neutrophils, as indicated by chemiluminescence, was found upregulated in peripheral blood $4 \mathrm{~h}$ after PDT [63], indicating this acute immune response might not be restricted to the treated lesion. In addition, after 48-72 h myeloid cells such as macrophages and mast cells accumulate at the treated site [62]. Increased expression of immunostimulatory IL-23, IL-22, IL-17, and IFN- $\gamma$ was found in peritumoral inflammatory cells shortly after PDT [64]. Moreover, serum levels of a immunosuppressive cytokine TGF- $\beta$ were shown to decline at $4 \mathrm{~h}$ after PDT [63]. Together, these clinical data support the stimulatory effect of PDT on acute immune responses, which is consistent with the observations from preclinical studies [22$24,51,52]$. However, these responses seem to maintain within a week, after which they tend to decline, and become non-detectable after one month [64-66], suggesting a transient inflammatory response following PDT.

Likewise, increase of inflammation was also detected shortly after Photofrin-PDT, as indicated by elevated levels of serum IL-6, a pro-inflammatory cytokine, in patients with esophageal squamous cell carcinoma (ESCC) at one week after treatment, but this declined soon after two weeks [67]. In addition, a slight increase of peripheral neutrophils and monocytes was observed in these patients at one week. However, the late time point chosen in this study might not provide clear evidence of the initial rapid inflammation. Another interesting study involved Temoporfin-PDT in head and neck squamous cell carcinoma (HNSCC), where increased serum levels of IL-6 were detected with a peak at $24 \mathrm{~h}$, and HMGB1 with a peak at one week after treatment [68], further supporting PDT as a potent inducer of acute inflammation.

\section{Adaptive Immune Response}

Being potent APCs, DCs act as a central link with the adaptive immune response. Clinical data on DCs in PDT-induced immune responses are scarce, and mainly come from studies of BCC patients, where Langerhans cells were analyzed. Longo et al. found increase of Langerhans cells, accompanied with lymphocyte-like cells, at the tumor site one week after ALA-PDT [65]. In contrast, Evangelou et al. reported decrease of Langerhans cells at the treated site at 1 and $24 \mathrm{~h}$ after MAL-PDT [69]. This discrepancy could be explained by the difference in time point, treatment protocol, or way of measurement chosen in the study. On the other hand, the decrease of Langerhans within $24 \mathrm{~h}$ might be due to their migration from the tumor site to lymphoid organs, where they present antigens and subsequently activate $\mathrm{T}$ cell responses. Obviously, further study is warranted to elucidate this proposed mechanism.

The involvement of lymphocytes is supported by studies from BCC patients receiving ALA- or Photofrin-PDT. Increased lymphocytes at the tumor site were detected $24 \mathrm{~h}$ after ALA-PDT, and remained elevated for at least $72 \mathrm{~h}$, being CD4+ more abundant than CD8+ lymphocytes [62]. In another study, activity of peripheral lymphocyte activity was analyzed at $4 \mathrm{~h}$ after PDT, in which cellular expression of IL-1 $\beta$, IL-6, IL-2, and TNF- $\alpha$ was measured [63]. Nevertheless, only a slight decrease of IL-1 $\beta$ was detected, suggesting $4 \mathrm{~h}$ might not be the optimal time point to measure lymphocytes in peripheral blood. Of note, both Photofrin- and ALA-PDT enhanced lymphocyte recognition of a TAA hedgehog-interacting protein (Hip1) expressed in BCC [70]. Antigen recognition was significantly greater in patients whose lesions were treated with PDT, in comparison 
to surgical removal. The antigen-specific immune response described in this study is, most likely, mediated by CD8+ T cells since Hip1 peptide forms complexes with MHCI molecules. In supporting this, a study from patients with vulva intraepithelial neoplasia $(\mathrm{VIN})(\mathrm{n}=32)$ treated with ALA-PDT showed that VIN that display loss of MHC class I $(n=9)$ failed to respond to the treatment, whereas the responders exhibited significantly higher CD8+ $\mathrm{T}$ cell infiltration than non-responders [71]. In addition to $\mathrm{T}$ helper and cytotoxic lymphocytes, increasing number of regulatory T lymphocytes (Treg) were also observed in peripheral blood of patients receiving PDT treatments $[67,68]$.

\section{Systemic Immune Response}

Even though PDT is a treatment applied locally in cancer patients, available clinical data suggest its potential to trigger systemic immune responses, and in some cases even an abscopal effect. For instance, remission of tumors outside the treated area has been reported in several cases of BCC [70] or angiosarcoma [72], following the local treatment with ALA- or Fotolon-PDT, respectively. In the former study, the authors described that such effect was accompanied by an increased cytolytic activity of splenocytes and infiltration of CD8+ lymphocytes in untreated tumors [70]. Besides, supporting evidence also includes enhanced activity of immune cells in peripheral blood after local treatments of PDT, such as neutrophil [63] and lymphocyte activity [62,70] (see Section 3.1.1 and Section 3.1.2). In addition, NK cell numbers were found increased in peripheral blood of HNSCC after Temoporfin-PDT [68]. Treg isolated from peripheral blood exhibited reduced immunosuppressive activities in ESCC patients after Photofrin-PDT [67]. These clinical data are however scarce. As such, obtaining more evidence will contribute to a better understanding for such potential of PDT, and to ultimately being able to use the information for improving therapeutic outcomes.

\section{Potentiating PDT with Immune Modulation}

Despite much evidence showing immune stimulation after PDT, the generation of robust antitumor immune responses triggered by PDT is, however, not often the case [73]. This could be, at least partly, explained by the fact that tumors are heterogenous and exhibit different immunogenicity reflected by more or less immune cell infiltrates (also referred to as "hot" versus "cold" tumors). Another hurdle are loads of immunosuppressive factors present locally at the tumor site or systemically [74], which occurs often in advanced cancer patients [75]. Strategies by combining agents that boost the immune system and/or reverse the immunosuppression would, therefore, enhance the occurrence of effective and long-lasting immune responses against cancer, at the same time as PDT destroys the actual tumor. These include, but not limited to, various immunostimulants, blocking or depleting immunosuppressive (cellular) factors, inducing tumor antigens and immunepotentiating vaccines such as DC-based vaccines.

\section{Immunostimulants}

Being widely used as adjuvants for enhancing cancer vaccines, TLR agonists, such as Bacillus Calmette-Guérin (BCG, TLR-2/4), imiquimod (TLR-7), and CpG oligodeoxynucleotide (CpG ODN, TLR-9), are potent immune stimulants [76]. Through binding to PRRs on immune cells, they can improve antigen delivery, processing, and presentation by APCs, or induce immunomodulatory cytokines production [76]. It has been shown that administration of BCG increased the number of tumor-free mice after PDT, regardless of the type of PS employed, including Photofrin, benzoporphyrin 
derivative, Temoporfin, mono-L-aspartyl-chlorin e6, lutetium texaphyrin, or zinc phthalocyanine [31]. Interestingly, the ratio of memory T lymphocyte subsets is further increased at tumor lymph nodes in the combination with BCG, compared to PhotofrinPDT alone. The use of CpG ODN in conjunction with PDT has also been successfully demonstrated. For instance, the co-injection of $\mathrm{CpG}$ with Radachlorin-PDT-generated tumor lysates elicited a strong antitumor immune response, resulting in increased production of tumor-specific antibodies and cytotoxic $\mathrm{T}$ cell responses [77]. Besides, Verteporfin-PDT in combination with $\mathrm{CpG}$ demonstrated decreased tumor sizes and better survivals, compared to either treatment alone [78]. Topical PDT, generally applied to treat cancer limited to the skin surface, when co-applied with imiquimod cream, has been proved effective as well for invasive squamous cell carcinoma, in both mice models and humans [79]. Zymosan, being known as a TLR-2 agonist, has been shown to augment the cure rate of tumor after Photofrin-PDT, as well as the levels of C3 complement [80]. Other TLR agonists such as mycobacterium cell wall extract (MCWE) also demonstrated significant synergetic effects on cancer treatment with PDT using different PSs [81]. Recently, there has emerged a new class of immunoadjuvants, e.g., Ndihydrogalactochitosan - a semisynthetic cationic carbohydrate polymer and derivative of chitin (an abundant natural polysaccharide) exhibiting complex advantageous properties encompassing both immunostimulatory effectiveness and amplifying PDTmediated direct lethal cell kill [82].

A major sign for tumor ICD is the release of DAMPs, such as CRT, HSP70, HMGB1, or ATP, which through binding to PRRs can induce immune maturation and activation at the local site (as discussed in Section 3.1.3). In fact, recombinant CRT, upon peritumoral injection, has been shown to boost the therapeutic effect of PDT and/or PDTgenerated cancer vaccines [83]. In addition, immunostimulants such as glycated chitosan [84], vitamin D3-binding protein-derived macrophage-activating factor (DBPMAF) [85], neutrophil promoting factor G-CSF [34,86], and Schizophillan (SPG) [87] have shown beneficial effects in the case of Photofrin-PDT, and CCL8 (monocyte chemoattractant protein-2) in the case of ALA-PDT [88].

\section{Blocking or Depleting Immunosuppressive (Cellular) Factors}

Tumors that are resistant to first-line therapy (surgery, radiotherapy, and chemotherapy), or in advanced stages, often develop severe immunosuppressive burden. This is featured by upregulation of inhibitory molecules that restrain immune activation, signals that promote tumor growth and/or accumulation of immunosuppressive cells. Therefore, approaches that reduce/inhibit the immunosuppressive factors and restore the immune responses would, at large, improve the therapeutic outcomes of PDT in these cancers. An excellent example of this is the addition of immune checkpoint inhibitors (e.g., anti-PDL1 and anti-CTLA-4), which have revolutionized the treatment of various cancers. The blockade of PD-L1 or CTLA-4 restores the tumoricidal activity of lymphocytes, and, more recently, has also been shown to synergize the therapeutic effect of PDT, where abscopal effects were observed [89-92]. Interestingly, this synergistic effect is also observed with third-generation photosensitizers, i.e., antibody-photosensitizer conjugates for targeted PDT, resulting in increased tumor infiltration of mature DCs and T lymphocytes, abscopal antitumor effects, and immunologic memory [93,94]. Another possible mechanism of PDT resistance is the accumulation of immunosuppressive cells at the tumor sites, including Tregs and myeloid derived suppressive cells (MDSCs). These cells, through heterogenous mechanisms, exhibit a potent ability to inhibit several components and phases of immune responses $[95,96]$. Upon selective depletion of Tregs with low dose cyclophosphamide prior to PDT, a dramatically decreased tumor size and 
increased survival was observed [97]. In addition, depletion of MDSCs by using GR1 blocking antibody improved tumor cure rates of PDT. Such beneficial effect, nevertheless, disappeared when anti-GR1 was injected immediately, instead of $1 \mathrm{~h}$, after PDT illumination [98]. The abrogated therapeutic effect could be caused by the unwanted depletion of neutrophils, with anti-GR1 at the acute phase of PDT (i.e., within an hour), where neutrophils play an essential role in stimulating immune responses. In addition to cellular fractions, soluble mediators such as TGF- $\beta$ and PGE2 also contribute to the immunosuppressive tumor microenvironment [99]. As such, agents that inhibit these factors would also be beneficial to enhance the anti-tumor immune responses induced by PDT.

\section{Importance of Tumor Antigens}

Tumor antigens can be unique tumor-specific antigens [100], antigens that can be found in both normal and tumor tissues but are overexpressed in tumors [101], or antigens of viral etiology [102]. Recognition of tumor antigens is the first step for establishing longterm immune memory in cancer immunotherapy. As such, the type and degree of tumor antigen expression plays an essential role in the immune effects of PDT. It has been suggested that PDT-mediated ER stress and ROS production are likely to increase the expression and liberation of antigens [73], but it has never been investigated. In reality, most clinical tumors, however, exhibit weak immunogenicity and therefore limit the longterm effects of PDT. In addition, tumor cells may escape immune surveillance by losing the expression of tumor antigen or downregulation of MHC I molecules. Restoring or inducing antigen expression or presentation by the tumor cells is, therefore, key to tackling this type of tumors. Attempts have been made to induce expression of a silenced tumor antigen P1A with 5-Aza-2'-deoxycytidine (a methyltransferase inhibitor) in four different tumor models (Lewis lung carcinoma, 4T1 mammary carcinoma, CT26 colon carcinoma, and EMT6 mammary carcinoma) that are treated with Photofrin-PDT. Of particular interest, the combination strategy leads to complete tumor cures and long-term survival in CT26 and EMT6 models, and even resistance to the re-challenge with the same tumor cells [59]. Furthermore, these effects were largely dependent on CD8+ T lymphocytes.

\section{Immune Potentiating Therapeutic Vaccines}

Therapeutic vaccines against cancer are getting more attention since (neo)antigen identification has become more technically feasible in a time frame that allows detailed molecular analysis of the specific peptide sequences [103]. Several types of vaccines have been explored as well as combined with PDT. The use of DC-based vaccines for cancer has been extensively investigated, with more than 200 complete clinical trials to date [104]. Preclinical evidence demonstrate that PDT-treated tumors enhance DC recruitment, maturation, and cytokine secretion [45,54,105-107]. Indeed, using PDTtreated tumor cells as adjuvant for DC-based vaccine (PDT-DC vaccine) has been proved to trigger stronger protection against tumors $[12,108,109]$, and enhanced $\mathrm{T}$ lymphocyte responses [109], compared to using freeze/thaw-treated tumor cells. Furthermore, local PDT followed by intratumoral injection of DC $[10,110]$ has shown decreased tumor sizes, better survival, and higher cytotoxicity mediated by CD8+ T lymphocytes and NK cells [10], compared to either group alone. Using CT26 colorectal carcinoma and B16 melanoma mice models, Saji at al. showed this strategy significantly enhanced tumorcured rates in mice and prolonged the survival of mice of which the tumors were not cured [61]. Remarkably, this strategy induced regression of tumor at distant sites including lung 
metastases, implying the systemic antitumor effect of PDT could be greatly enhanced by such strategy.

Molecularly defined therapeutic peptide vaccination has been successfully combined with chlorin e6-based PDT in mouse models and convincingly shows synergistic clearance of primary tumors. Abscopal CD8 T cell-mediated effects were shown by reduction of secondary tumors [111]. These vaccines have been even further improved by conjugation to a defined DC-stimulatory TLR2 ligand, which showed strong anti-tumor effects when combined with PDT [112].

In conclusion, a better understanding of the tumor microenvironment and the development of current immunotherapies have provided a wealth of opportunities for devising combination strategies to trigger robust immune responses after PDT. For instance, approaches that reverse hypoxia at the tumor site [113] would likely enhance the PDT-induced immune responses, since severe hypoxia contributes to the tumor immunosuppression, and also adequate oxygen levels are critical for PDT efficacy. However, human bodies are far more complicated and heterogenous than laboratory models. Therefore, efforts are ongoing towards a better preclinical model as close as possible to patients, such as humanized mouse models engrafted with patient-derived cancer organoids and immune system. Besides, the PDT parameters such as choice of PS, doses of both PS and illumination, fluence rate, and drug-light interval are also important in optimizing the immune responses.

\section{Future Directions for Clinical Research}

Preclinical studies point at PDT as very effective in breaking the immunotolerance in treated tumors, overturning the immunosuppressive tumor microenvironment, and instigating the development of a strong adaptive immune response against these lesions. However, scarce information has been provided concerning these events in the clinical setting (see Table 3 for an overview of the reviewed clinical studies). Analysis of the literature in this case is limited due to the low number of clinical studies. With this in mind, suggestions are given in this section that could contribute to the collection of additional and valuable information from future clinical studies, as well as promote further understanding and exploration of the implication of the immune system for the greater success of the PDT treatment.

\section{Recommendations for Immune Monitoring}

Proper immune monitoring of the initial innate effects, as well as the downstream effects on the adaptive response, is key to designing successful combination strategies. Numerous clinical trials focus on the efficacy of the PDT treatment, but little can be found on its effects on the immune system. In general, the available clinical evidence seems to correlate with preclinical data, but this evidence is limited, and further study is needed to safely claim correlation. If we are to learn from preclinical studies, most significant changes in cells and mediators occur during the first day (innate response) and first week (adaptive response) after PDT. Based on the reviewed preclinical and clinical data, a timeline is shown in Figure 2 as an indication of the main immunological events developed post-PDT. This timeline might serve as guidance when designing new clinical studies aiming to further characterize the immune response after PDT. 


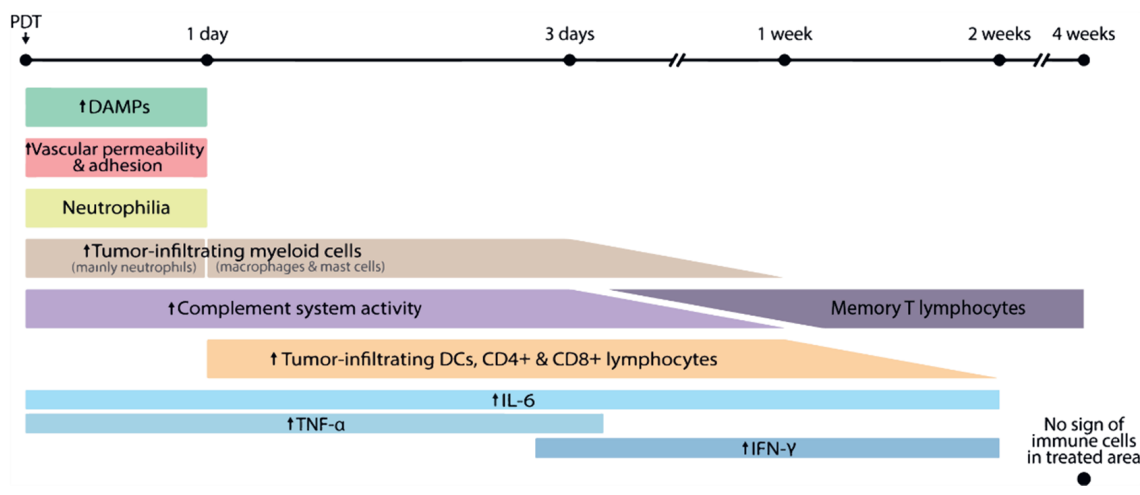

Figure 2 Indicative timeline of the main immunological events developed after PDT. Shortly after PDT, an initial inflammatory response arises due to the exposure of DAMPs and complement activation, and is facilitated by the increased vascular adhesion and permeabilization at the tumor site during the first $24 \mathrm{~h}$ post-PDT. A pronounced neutrophilia occurs, which is followed by increased numbers of tumor-infiltrating neutrophils during the first $24 \mathrm{~h}$, and infiltrating macrophages and mast cells at least during the first $72 \mathrm{~h}$. One day post-PDT, DCs and lymphocytes start accumulating at the tumor site. This triggered adaptive response might last for two weeks and is accompanied by high levels of IL- 6 , TNF- $\alpha$, and IFN- $\gamma$, as well as elevated complement activity. The initial immunological response is transient and four weeks post-PDT immune cells are no longer detected in the treated area. After this initial response, tumor-specific memory effector $\mathrm{T}$ cells can be expected to be present in circulation or in the tumor-draining lymphoid organs.

Human studies could first focus on the confirmation of the most determinant immune events observed in animals. Certain cytokines, i.e., IFN- $\gamma$, TNF- $\alpha$, and IL-6, have been found to play important roles in preclinical studies, particularly during the initial inflammation and the transition to a $\mathrm{T}$ cell-mediated response during the first week postPDT [29,51]. Special attention should be paid to IL-6 due to its tumor promoting role observed in mice under particular PDT regimens [114,115]. In fact, high levels of IL-6 have been reported within the first two weeks after clinical PDT [67]. As for the cellular components mediating the immune response, a marked neutrophilia [34], maturation of DCs [51], elevated numbers of T cells [116], and increased cytotoxic activity of CD8+ T cells [59] are features observed after preclinical PDT. Therefore, detection and study of these cell types would be a logical starting point in patients.

The effects of PDT on the adaptive response, especially tumor-specific $\mathrm{T}$ cell expansions which are observed in preclinical models in lymphoid organs and in blood, allows monitoring of patients by collecting blood samples before and after therapy. Sustainment of tumor-specific effector memory $\mathrm{T}$ cells is important for cures and systemic control of recurrences; thus, follow-up blood sampling is recommended. Furthermore, as cancer type related epitope knowledge and identification of new tumorspecific $T$ cell specificities will be increasing in the near future, monitoring of tumorspecific $\mathrm{T}$ cell responses by high-end flow cytometry will become feasible. Modern technologies to identify patient-specific neo-epitopes by exome sequencing and HLAbinding algorithms will become more rapid and applicable for individual patient monitoring. Moreover, quantitative analysis of the levels of circulating specific T cells but also the monitoring of the quality of the $\mathrm{T}$ cell response by analysis of cytokine profiles and cell surface markers for effector, memory, or regulatory $\mathrm{T}$ cell subpopulations are essential. This information could decisively influence further $\mathrm{T}$ cell 
directed treatment options to be combined with PDT (e.g., specific stimulation with agonistic antibodies or stimulatory vaccines). In addition, following levels of other immune cell types which have remained relatively unexplored in animals, such as NK cells, B cells, and Treg cells, would allow further characterization of the response. Once the critical immune elements in humans have been characterized, and when necessary, immunomodulatory agents can be specifically and wisely applied in combination with PDT to maximize efficacy of the treatment.

While preclinical studies allow a broad use of techniques to modify the animal in order to characterize a particular process in detail, this is limited in the case of humans. Non-invasive methods in the clinic for detection and measurement of the immune response after PDT are of extreme value, and development of such techniques would greatly add to the understanding of this process. The obvious current choice is the collection of blood for systemic analysis, as highlighted above, and some studies point at reflectance confocal microscopy for examining the tumor site in the case of superficial skin cancers [65]. This imaging tool allows monitoring of the tumor response as well as detection of immune cells at the tumor over time. The activity of the different immune cells isolated from patients can be evaluated ex vivo and correlated with changes in other cellular or soluble components, as well as with the clinical outcome in order to be able to make predictions based on correlations. It is also of interest to study the response of cancer lesions outside the treated area. This is an unexplored event in the clinic (with exception of case reports), relatively easy to monitor and of great clinical value. Study of the immune response as here described can be performed as secondary endpoints in clinical trials focused on PDT safety and efficacy.

\section{Recommendations for Optimizing PDT Outcome}

PDT is now firmly stablished as able to render an anti-tumor immune response; however, this does not necessarily equal to tumor cure. To achieve this, it has to be ensured that the elicited immune response remains vigorous and durable. Future preclinical and clinical research has to optimize means of preventing that the elicited immune response remains incomplete and stalls before the immune destruction of both local and distant tumor deposits is complete. To meet this goal, we review here the use of a series of PDT adjuvants and joint agents based on their potential for preventing immunoregulatory mechanisms known to hinder the effectiveness of PDT. We strongly believe that further research along these lines can certainly boost the potential of PDT to its maximum. While considerable advances were made in developing and characterizing PDT-generated cancer vaccines in preclinical studies, very little effort has been made in translating this into the clinic and establishing the benefits of PDT vaccines with cancer patients. It seems likely that clinical results can turn out to be even more positive than predicated with fast growing animal models, especially with (thus far untested) full scale multiple vaccination regimens and combination with adjuvants preventing development of immunosuppressive microenvironment.

Preclinical as well as clinical evidence indicates that the illumination conditions have an important impact on the activation of the immune system and overall success of the PDT therapy [70,117-119]. It has been suggested that high fluence rates deplete oxygen levels at the tumor site in patients with BCC [119], resulting in a possibly inefficient treatment although still effective due to the extensive vascular and tumor damage. Moreover, high fluences seem to have a negative impact on immune reactivity towards tumor antigens in patients with BCC [70], supporting the fact that high fluences may not be ideal for the development of an immune response, likely due to a potent vascular shutdown that prevents immune cells from reaching the tumor area. On the other 
hand, preclinical studies point to the fact that low fluences with low fluence rates inflict diffuse damage and facilitate the enhancement of an inflammatory response, but may yield lower cure rates [120].

The illumination protocols commonly used in the clinic are, in general, devised to induce extensive damage to the primary tumor, while the immunobiology of PDT is overlooked. Based on the reviewed clinical studies, immunostimulatory PDT regimens consist of moderate to high fluences, low fluence rates, and treatment of small surface areas. These illumination regimens might be especially beneficial when combined in the clinic with immunostimulatory strategies. Interestingly, a two-fold fractionated illumination scheme (low fluence followed by high fluence) has shown superior efficacy than single illumination to treat patients with superficial BCC [121]. This suggests great clinical benefit can arise from the design of two-step PDT programs consisting of a first immune-stimulating illumination regimen, followed by a regimen that mediates potent tumor destruction, which has proven favorable in the preclinical setting [117].

All things considered, further preclinical exploration of the different PDT regimens is still essential. Due to the critical role of the illumination parameters, we would like to underline the importance of these being always specified in published studies. Likewise, we also highlight the importance of stratifying patients based on previous received treatments, such as radio-, chemo- and adjuvant therapy. These, upon repeated use, can have a large effect on the immune system, leading to exhausted immune responses or immunosuppression. To our surprise, four out of the 10 reviewed clinical studies have not addressed this aspect, and only three of these 10 gave additional details regarding prior treatments.

\section{Conclusions}

Many studies here reviewed document the activation of the immune system postoncologic PDT. Preclinical studies point at PDT as very effective in breaking the immunotolerance in treated tumors, disturbing the immunosuppressive tumor microenvironment and developing a strong and systemic adaptive immune response against even distant tumor lesions. Clinical evidence, however, is unfortunately very scarce. We believe that collecting more of this evidence in the clinical setting will contribute to the full understanding of these events in humans. Consequently, this would enable the design of new trials in which the immunological potential of PDT is explored in its full, for more effective treatment of many cancer patients.

\section{References}

1. van Straten, D.; Mashayekhi, V.; de Bruijn, H.S.; Oliveira, S.; Robinson, D.J. Oncologic photodynamic therapy: Basic principles, current clinical status and future directions. Cancers (Basel). 2017, 9, 1-54.

2. Velez-montoya, R.; Oliver, S.C.N.; Olson, J.L.; Fine, S.L.; Mandava, N.; Quiroz-mercado, H. Current knowledge and trends in age-related macular degeneration: today's and future treatments. Retina 2013, 33, 1487-1502.

3. Reinhard, A.; Sandborn, W.; Melhem, H.; Bolotine, L.; Chamaillard, M.; Peyrin-Biroulet, L. Photodynamic therapy as a new treatment modality for inflammatory and infectious conditions. Expert Rev. Clin. Immunol. 2015, 11, 637-657.

4. U.S. National Institutes of Health ClinicalTrials.gov Available online: https:/clinicaltrials.gov/ (accessed on May 1, 2020). 
5. Chen, B.; Pogue, B.; Hoopes, P.; Hasan, T. Vascular and cellular targeting for photodynamic therapy. Crit. Rev. Eukaryot. Gene Expr. 2006, 16, 279-305.

6. Agostinis, P.; Berg, K.; Cengel, K..; Foster, T..; Girotti, A..; Gollnick, S..; Hahn, S..; Hamblin, M..; Juzeniene, A.; Kessel, D.; et al. Photodynamic Therapy of cancer: an update. CA Cancer J Clin. 2011, 61, 250-281.

7. Castano, A.P.; Demidova, T.N.; Hamblin, M.R. Mechanisms in photodynamic therapy: Part threePhotosensitizer pharmacokinetics, biodistribution, tumor localization and modes of tumor destruction. Photodiagnosis Photodyn. Ther. 2005, 2, 91-106.

8. Garg, A.D.; Nowis, D.; Golab, J.; Agostinis, P. Photodynamic therapy: Illuminating the road from cell death towards anti-tumour immunity. Apoptosis 2010, 15, 1050-1071.

9. Garg, A.D.; Agostinis, P. ER stress, autophagy and immunogenic cell death in photodynamic therapy-induced anti-cancer immune responses. Photochem. Photobiol. Sci. 2014, 13, 474- 487

10. Jalili, A.; Makowski, M.; Switaj, T.; Nowis, D.; Wilczek, E.; Chora, M. Effective Photoimmunotherapy of Murine Colon Carcinoma Induced by the Combination of Photodynamic Therapy and Dendritic Cells. Clin. Cancer Res. 2004, 10, 4498-4508.

11. Korbelik, M.; Sun, J.; Cecic, I. Photodynamic Therapy - Induced Cell Surface Expression and Release of Heat Shock Proteins : Relevance for Tumor Response. Cancer Res. 2005, 65, 1018 1026.

12. Ji, J.; Fan, Z.; Zhou, F.; Wang, X.; Shi, L.; Zhang, H.; Wang, P.; Yang, D.; Zhang, L.; Chen, W.R.; et al. Improvement of DC vaccine with ALA-PDT induced immunogenic apoptotic cells for skin squamous cell carcinoma. Oncotarget 2015, 6, 17135-17146.

13. Korbelik, M. Role of cell stress signaling networks in cancer cell death and antitumor immune response following proteotoxic injury inflicted by photodynamic therapy. Lasers Surg. Med. 2018, $50,491-498$.

14. Díaz-Villanueva, J.F.; Díaz-Molina, R.; García-González, V. Protein folding and mechanisms of proteostasis. Int. J. Mol. Sci. 2015, 16, 17193-230.

15. Hetz, C. The unfolded protein response: Controlling cell fate decisions under ER stress and beyond. Nat. Rev. Mol. Cell Biol. 2012, 13, 89-102.

16. van Vliet, A.R.; Martin, S.; Garg, A.D.; Agostinis, P. The PERKs of damage-associated molecular patterns mediating cancer immunogenicity: From sensor to the plasma membrane and beyond. Semin. Cancer Biol. 2015, 33, 74-85.

17. Janssens, S.; Pulendran, B.; Lambrecht, B.N. Emerging functions of the unfolded protein response in immunity. Nat. Immunol. 2014, 15, 910-919.

18. Starck, S.R.; Tsai, J.C.; Chen, K.; Shodiya, M.; Wang, L.; Yahiro, K.; Martins-Green, M.; Shastri, N.; Walter, P. Translation from the 5' untranslated region shapes the integrated stress response. Science. 2016.

19. Starck, S.R.; Shastri, N. Nowhere to hide: unconventional translation yields cryptic peptides for immune surveillance. Immunol. Rev. 2016, 272, 8-16.

20. Keisari, Y. Tumor abolition and antitumor immunostimulation by physico-chemical tumor ablation. Front. Biosci. Landmark 2017, 22, 310-347.

21. Seitz, C.; Rückert, M.; Deloch, L.; Weiss, E.-M.; Utz, S.; Izydor, M.; Ebel, N.; Schlücker, E.; Fietkau, R.; Gaipl, U.S.; et al. Tumor Cell-Based Vaccine Generated With High Hydrostatic Pressure Synergizes With Radiotherapy by Generating a Favorable Anti-tumor Immune Microenvironment. Front. Oncol. 2019, 9, 805.

22. Krosl, G.; Korbelik, M.; Dougherty, G.J. Induction of immune cell infiltration into murine SCCVII tumour by photofrin-based photodynamic therapy. Br. J. Cancer 1995, 71, 549-555.

23. Davis, R.W.; Snyder, E.; Miller, J.; Carter, S.; Houser, C.; Klampatsa, A.; Albelda, S.M.; Cengel, K.A.; Busch, T.M. Luminol Chemiluminescence Reports Photodynamic Therapy-Generated Neutrophil Activity In Vivo and Serves as a Biomarker of Therapeutic Efficacy. Photochem. Photobiol. 2019, 95, 430-438.

24. Sun, J.; Korbelik, M. Role of Complement Anaphylatoxin C3a in Photodynamic Therapy-elicited Engagement of Host Neutrophils and Other Immune Cells. Photochem. Photobiol. 2006, 82, 558- 
562.

25. Cecic, I.; Parkins, C.S.; Korbelik, M.; Cecic, I.; Parkins, C.S.; Korbelik, M. Induction of Systemic Neutrophil Response in Mice by Photodynamic Therapy of Solid Tumors. Photochem. Photobiol. 2001, 74, 712-720.

26. Cecic, I.; Stott, B.; Korbelik, M. Acute phase response-associated systemic neutrophil mobilization in mice bearing tumors treated by photodynamic therapy. Int. Immunopharmacol. 2006, 6, 1259 1266.

27. Vree, W.J. a De; Essers, M.C.; Koster, J.F.; Koster, F. Role of Interleukin 1 and Granulocyte Colony-Stimulating Factor in Photofrin-based Photodynamic Therapy of Rat Rhabdomyosarcoma Tumors. Cancer Res. 1997, 57, 2555-2558.

28. de Bruijn, H.S.; Sluiter, W.; van der Ploeg-van den Heuvel, A.; Sterenborg, H.J.C.M.; Robinson, D.J. Evidence for a bystander role of neutrophils in the response to systemic 5-aminolevulinic acidbased photodynamic therapy. Photodermatol. Photoimmunol. Photomed. 2006, 22, 238-246.

29. Cecic, I.; Korbelik, M. Mediators of peripheral blood neutrophilia induced by photodynamic therapy of solid tumors. Cancer Lett. 2002, 183, 43-51.

30. Sun, J.; Cecic, I.; Parkins, C.S.; Korbelik, M. Neutrophils as inflammatory and immune effectors in photodynamic therapy-treated mouse SCCVII tumours. Photochem. Photobiol. Sci. 2002, 1, 690-695.

31. Korbelik, M.; Sun, J.; Posakony, J.J. Interaction between photodynamic therapy and BCG immunotherapy responsible for the reduced recurrence of treated mouse tumors. Photochem. Photobiol. 2001, 73, 403-409.

32. Gollnick, S.O.; Evans, S.S.; Baumann, H.; Owczarczak, B.; Maier, P.; Vaughan, L.; Wang, W.C.; Unger, E.; Henderson, B.W. Role of cytokines in photodynamic therapy-induced local and systemic inflammation. Br. J. Cancer 2003, 88, 1772-1779.

33. Schacht, V.; Szeimies, R.-M.; Abels, C. Photodynamic therapy with 5-aminolevulinic acid induces distinct microcirculatory effects following systemic or topical application. Photochem. Photobiol. Sci. 2006, 5, 452-458.

34. Vree, W.J. a De; Essers, M.C.; Bruijn, H.S. De; Star, W.M.; Koster, J.F.; Sluiter, W. Evidence for an Important Role of Neutrophils in the Efficacy of Photodynamic Therapy in Vivo. Cancer Res. 1996, 56, 2908-2911.

35. Mamidi, S.; Höne, S.; Kirschfink, M. The complement system in cancer: Ambivalence between tumour destruction and promotion. Immunobiology 2017, 222, 45-54.

36. Cecic, I.; Serrano, K.; Gyongyossy-issa, M.; Korbelik, M. Characteristics of complement activation in mice bearing Lewis lung carcinomas treated by photodynamic therapy. Cancer Lett. 2005, 225, 215-223.

37. Korbelik, M.; Cecic, I. Complement activation cascade and its regulation: Relevance for the response of solid tumors to photodynamic therapy. J. Photochem. Photobiol. B Biol. 2008, 93, 5359.

38. Casares, N.; Pequignot, M.O.; Tesniere, A.; Ghiringhelli, F.; Roux, S.; Chaput, N.; Schmitt, E.; Hamai, A.; Hervas-Stubbs, S.; Obeid, M.; et al. Caspase-dependent immunogenicity of doxorubicin-induced tumor cell death. J. Exp. Med. 2005, 202, 1691-1701.

39. Obeid, M.; Tesniere, A.; Ghiringhelli, F.; Fimia, G.M.; Apetoh, L.; Perfettini, J.L.; Castedo, M.; Mignot, G.; Panaretakis, T.; Casares, N.; et al. Calreticulin exposure dictates the immunogenicity of cancer cell death. Nat. Med. 2007, 13, 54-61.

40. Garg, A.D.; Vandenberk, L.; Fang, S.; Fasche, T.; Van Eygen, S.; Maes, J.; Van Woensel, M.; Koks, C.; Vanthillo, N.; Graf, N.; et al. Pathogen response-like recruitment and activation of neutrophils by sterile immunogenic dying cells drives neutrophil-mediated residual cell killing. Cell Death Differ. 2017, 24, 832-843.

41. Adkins, I.; Fucikova, J.; Garg, A.D.; Agostinis, P.; Špíšek, R. Physical modalities inducing immunogenic tumor cell death for cancer immunotherapy. Oncoimmunology 2014, 3.

42. Bezu, L.; Sauvat, A.; Humeau, J.; Leduc, M.; Kepp, O.; Kroemer, G. eIF2 $\alpha$ phosphorylation: A hallmark of immunogenic cell death. Oncoimmunology 2018, 7, 1-3. 
43. Garg, A.D.; Dudek, A.M.; Agostinis, P. Cancer immunogenicity, danger signals, and DAMPs: What, when, and how? Biofactors 2013, 39, 355-367.

44. Korbelik, M.; Zhang, W.; Merchant, S. Involvement of damage-associated molecular patterns in tumor response to photodynamic therapy: Surface expression of calreticulin and high-mobility group box-1 release. Cancer Immunol. Immunother. 2011, 60, 1431-1437.

45. Wang, X.; Ji, J.; Zhang, H.; Fan, Z.; Zhang, L.; Shi, L.; Zhou, F.; Chen, W.R.; Wang, H.; Wang, $\mathrm{X}$. Stimulation of dendritic cells by DAMPs in ALA-PDT treated SCC tumor cells. Oncotarget 2015, 6, 44688-44702.

46. Kroemer, G.; Galluzzi, L.; Kepp, O.; Zitvogel, L. Immunogenic Cell Death in Cancer Therapy. Annu. Rev. Immunol. 2013.

47. Humeau, J.; Lévesque, S.; Kroemer, G.; Pol, J.G. Gold standard assessment of immunogenic cell death in oncological mouse models. In Methods in Molecular Biology; 2019.

48. Gollnick, S.O.; Vaughan, L.; Henderson, B.W. Generation of Effective Antitumor Vaccines Using Photodynamic Therapy. Cancer Res. 2002, 62, 1604-1608.

49. Zhou, F.; Xing, D.; Chen, W.R. Regulation of HSP70 on activating macrophages using PDTinduced apoptotic cells. Int. J. Cancer 2009, 125, 1380-1389.

50. Korbelik, M. Cancer vaccines generated by photodynamic therapy. Photochem. Photobiol. Sci. 2011.

51. Gollnick, S.O.; Owczarczak, B.; Maier, P. Photodynamic therapy and anti-tumor immunity. Lasers Surg. Med. 2006, 38, 509-515.

52. Wang, H.; Li, J.; Lv, T.; Tu, Q.; Huang, Z.; Wang, X. Therapeutic and immune effects of 5aminolevulinic acid photodynamic therapy on UVB-induced squamous cell carcinomas in hairless mice. Exp. Dermatol. 2013, 22, 358-379.

53. Audiger, C.; Rahman, M.J.; Yun, T.J.; Tarbell, K.V.; Lesage, S. The Importance of Dendritic Cells in Maintaining Immune Tolerance. J. Immunol. 2017.

54. Etminan, N.; Peters, C.; Lakbir, D.; Bünemann, E.; Börger, V.; Sabel, M.C.; Hänggi, D.; Steiger, H.J.; Stummer, W.; Sorg, R.V. Heat-shock protein 70-dependent dendritic cell activation by 5aminolevulinic acid-mediated photodynamic treatment of human glioblastoma spheroids in vitro. Br. J. Cancer 2011, 105, 961-969.

55. Korbelik, M.; Dougherty, G.J. Photodynamic therapy-mediated immune response against subcutaneous mouse tumors. Cancer Res. 1999, 59, 1941-1946.

56. Korbelik, M.; Krosl, G.; Krosl, J.; Dougherty, G.J. The role of host lymphoid populations in the response of mouse EMT6 tumor to photodynamic therapy. Cancer Res. 1996, 56, 5647-5652.

57. Korbelik, M.; Cecic, I. Contribution of myeloid and lymphoid host cells to the curative outcome of mouse sarcoma treatment by photodynamic therapy. Cancer Lett. 1999, 137, 91-98.

58. Kabingu, E.; Vaughan, L.; Owczarczak, B.; Ramsey, K.; Gollnick, S. CD8+ T cell-mediated control of distant tumours following local photodynamic therapy is independent of CD4+ T cells and dependent on natural killer cells. Br. J. Cancer 2007, 96, 1839-1848.

59. Wachowska, M.; Gabrysiak, M.; Muchowicz, A.; Bednarek, W.; Barankiewicz, J.; Rygiel, T.; Boon, L.; Mroz, P.; Hamblin, M.R.; Golab, J. 5-Aza-2'-deoxycytidine potentiates antitumour immune response induced by photodynamic therapy. Eur. J. Cancer 2014, 50, 1370-1381.

60. Kabingu, E.; Vaughan, L.; Owczarczak, B.; Ramsey, K.; Gollnick, S. CD8+ T cell-mediated control of distant tumours following local photodynamic therapy is independent of CD4+ T cells and dependent on natural killer cells. Br. J. Cancer 2007, 96, 1839-1848.

61. Li, Z.; Wang, C.; Deng, H.; Wu, J.; Huang, H.; Sun, R.; Zhang, H.; Xiong, X.; Feng, M. Robust Photodynamic Therapy Using 5-ALA-Incorporated Nanocomplexes Cures Metastatic Melanoma through Priming of CD4 + CD8 + Double Positive T Cells. Adv. Sci. 2019.

62. Zhang, H.; Wang, P.; Wang, X.; Shi, L.; Fan, Z.; Zhang, G.; Yang, D.; Bahavar, C.F.; Zhou, F.; Chen, W.R.; et al. Antitumor effects of DC vaccine with ALA-PDT-induced immunogenic apoptotic cells for skin squamous cell carcinoma in mice. Technol. Cancer Res. Treat. 2018, 17, 110. 
63. Prignano, F.; Lotti, T.; Spallanzani, A.; Berti, S.; De Giorgi, V.; Moretti, S. Sequential effects of photodynamic treatment of basal cell carcinoma. J. Cutan. Pathol. 2009, 36, 409-416.

64. Adamek, M.; Kawczyk-Krupka, A.; Mostowy, A.; Czuba, Z.; Krol, W.; Kasperczyk, S.; Jakobisiak, M.; Golab, J.; Sieron, A. Topical ALA-PDT modifies neutrophils' chemiluminescence, lymphocytes' interleukin-1beta secretion and serum level of transforming growth factor beta1 in patients with nonmelanoma skin malignancies: A clinical study. Photodiagnosis Photodyn. Ther. 2005, 2, 65-72.

65. Pellegrini, C.; Orlandi, A.; Costanza, G.; Di Stefani, A.; Piccioni, A.; Di Cesare, A.; Chiricozzi, A.; Ferlosio, A.; Peris, K.; Fargnoli, M.C. Expression of IL-23/Th17-related cytokines in basal cell carcinoma and in the response to medical treatments. PLoS One 2017.

66. Longo, C.; Casari, A.; Pepe, P.; Moscarella, E.; Zalaudek, I.; Argenziano, G.; Pellacani, G. Confocal microscopy insights into the treatment and cellular immune response of Basal cell carcinoma to photodynamic therapy. Dermatology 2012, 225, 264-270.

67. Winters, U.; Daayana, S.; Lear, J.T.; Tomlinson, A.E.; Elkord, E.; Stern, P.L.; Kitchener, H.C. Clinical and immunologic results of a phase II trial of sequential imiquimod and photodynamic therapy for vulval intraepithelial neoplasia. Clin. Cancer Res. 2008.

68. Reginato, E.; Lindenmann, J.; Langner, C.; Schweintzger, N.; Bambach, I.; Smolle-Jüttner, F.; Wolf, P. Photodynamic therapy downregulates the function of regulatory T cells in patients with esophageal squamous cell carcinoma. Photochem. Photobiol. Sci. 2014, 13, 1281-1289.

69. Theodoraki, M.N.; Lorenz, K.; Lotfi, R.; Fürst, D.; Tsamadou, C.; Jaekle, S.; Mytilineos, J.; Brunner, C.; Theodorakis, J.; Hoffmann, T.K.; et al. Influence of photodynamic therapy on peripheral immune cell populations and cytokine concentrations in head and neck cancer. Photodiagnosis Photodyn. Ther. 2017, 19, 194-201.

70. Evangelou, G.; Farrar, M.D.; Cotterell, L.; Andrew, S.; Tosca, a. D.; Watson, R.E.B.; Rhodes, L.E. Topical photodynamic therapy significantly reduces epidermal Langerhans cells during clinical treatment of basal cell carcinoma. Br. J. Dermatol. 2012, 166, 1112-1115.

71. Kabingu, E.; Oseroff, A.; Wilding, G.; Gollnick, S. Enhanced systemic immune reactivity to a Basal cell carcinoma associated antigen following photodynamic therapy. Clin. cancer Res. 2009, 15, 4460-4466.

72. Abdel-Hady, E.S.; Martin-Hirsch, P.; Duggan-Keen, M.; Stern, P.L.; Moore, J. V.; Corbitt, G.; Kitchener, H.C.; Hampson, I.N. Immunological and viral factors associated with the response of vulval intraepithelial neoplasia to photodynamic therapy. Cancer Res. 2001

73. Thong, P.S.P.; Ong, K.W.; Goh, N.S.G.; Kho, K.W.; Manivasager, V.; Bhuvaneswari, R.; Olivo, M.; Soo, K.C. Photodynamic-therapy-activated immune response against distant untreated tumours in recurrent angiosarcoma. Lancet Oncol. 2007.

74. Anzengruber, F.; Avci, P.; De Freitas, L.F.; Hamblin, M.R. T-cell mediated anti-tumor immunity after photodynamic therapy: Why does it not always work and how can we improve it? Photochem. Photobiol. Sci. 2015.

75. Chen, D.S.; Mellman, I. Oncology meets immunology: The cancer-immunity cycle. Immunity 2013.

76. Kalathil, S.G.; Thanavala, Y. High immunosuppressive burden in cancer patients: a major hurdle for cancer immunotherapy. Cancer Immunol. Immunother. 2016.

77. Circelli, L.; Tornesello, M.; Buonaguro, F.M.; Buonaguro, L. Use of adjuvants for immunotherapy. Hum. Vaccines Immunother. 2017.

78. Bae, S.M.; Kim, Y.W.; Kwak, S.Y.; Kim, Y.W.; Ro, D.Y.; Shin, J.C.; Park, C.H.; Han, S.J.; Oh, C.H.; Kim, C.K.; et al. Photodynamic therapy-generated tumor cell lysates with CpGoligodeoxynucleotide enhance immunotherapy efficacy in human papillomavirus 16 (E6/E7) immortalized tumor cells. Cancer Sci. 2007.

79. Xia, Y.; Gupta, G.K.; Castano, A.P.; Mroz, P.; Avci, P.; Hamblin, M.R. CpG oligodeoxynucleotide as immune adjuvant enhances photodynamic therapy response in murine metastatic breast cancer. J. Biophotonics 2014.

80. Bhatta, A.K.; Wang, P.; Keyal, U.; Zhao, Z.; Ji, J.; Zhu, L.; Wang, X.; Zhang, G. Therapeutic effect 
of Imiquimod enhanced ALA-PDT on cutaneous squamous cell carcinoma. Photodiagnosis Photodyn. Ther. 2018.

81. Korbelik, M.; Sun, J.; Cecic, I.; Serrano, K. Adjuvant treatment for complement activation increases the effectiveness of photodynamic therapy of solid tumors. Photochem. Photobiol. Sci. 2004, 3, 812-816.

82. Korbelik, M.; Cecic, I. Enhancement of tumour response to photodynamic therapy by adjuvant mycobacterium cell-wall treatment. J. Photochem. Photobiol. B Biol. 1998, 44, 151-158.

83. Korbelik, M.; Banáth, J.; Zhang, W.; Gallagher, P.; Hode, T.; Lam, S.S.K.; Chen, W.R. Ndihydrogalactochitosan as immune and direct antitumor agent amplifying the effects of photodynamic therapy and photodynamic therapy-generated vaccines. Int. Immunopharmacol. 2019.

84. Korbelik, M.; Banáth, J.; Saw, K.M.; Zhang, W.; Čiplys, E. Calreticulin as cancer treatment adjuvant: Combination with photodynamic therapy and photodynamic therapy-generated vaccines. Front. Oncol. 2015.

85. Chen, W.R.; Korbelik, M.; Bartels, K.E.; Liu, H.; Sun, J.; Nordquist, R.E. Enhancement of Laser Cancer Treatment by a Chitosan-derived Immunoadjuvant 1 . Photochem. Photobiol. 2005.

86. Korbelik, M.; Naraparaju, V.R.; Yamamoto, N. Macrophage-directed immunotherapy as adjuvant to photodynamic therapy of cancer. Br. J. Cancer 1997.

87. Gołab, J.; Wilczyński, G.; Zagozdzon, R.; Stokłosa, T.; Dabrowska, A.; Rybczyńska, J.; Wasik, M.; Machaj, E.; Ołda, T.; Kozar, K.; et al. Potentiation of the anti-tumour effects of Photofrin-based photodynamic therapy by localized treatment with G-CSF. Br. J. Cancer 2000, 82, 1485-1491.

88. Krosl, G.; Korbelik, M. Potentiation of photodynamic therapy by immunotherapy: the effect of schizophyllan (SPG). Cancer Lett. 1994, 84, 43-49.

89. Ji, J.; Wang, P.; Zhou, Q.; Zhu, L.; Zhang, H.; Zhang, Y.; Zheng, Z.; Bhatta, A.K.; Zhang, G.; Wang, X. CCL8 enhances sensitivity of cutaneous squamous cell carcinoma to photodynamic therapy by recruiting M1 macrophages. Photodiagnosis Photodyn. Ther. 2019.

90. Duan, X.; Chan, C.; Guo, N.; Han, W.; Weichselbaum, R.R.; Lin, W. Photodynamic Therapy Mediated by Nontoxic Core-Shell Nanoparticles Synergizes with Immune Checkpoint Blockade To Elicit Antitumor Immunity and Antimetastatic Effect on Breast Cancer. J. Am. Chem. Soc. 2016.

91. O’Shaughnessy, M.J.; Murray, K.S.; La Rosa, S.P.; Budhu, S.; Merghoub, T.; Somma, A.; Monette, S.; Kim, K.; Corradi, R.B.; Scherz, A.; et al. Systemic antitumor immunity by PD-1/PD-L1 inhibition is potentiated by vascular-targeted photodynamic therapy of primary tumors. Clin. Cancer Res. 2018.

92. Kleinovink, J.W.; Fransen, M.F.; Löwik, C.W.; Ossendorp, F. Photodynamic-immune checkpoint therapy eradicates local and distant tumors by CD8+ T cells. Cancer Immunol. Res. 2017.

93. Muchowicz, A.; Wachowska, M.; Stachura, J.; Tonecka, K.; Gabrysiak, M.; Wołosz, D.; Pilch, Z.; Kilarski, W.W.; Boon, L.; Klaus, T.J.; et al. Inhibition of lymphangiogenesis impairs antitumour effects of photodynamic therapy and checkpoint inhibitors in mice. Eur. J. Cancer 2017.

94. Bao, R.; Wang, Y.; Lai, J.; Zhu, H.; Zhao, Y.; Li, S.; Li, N.; Huang, J.; Yang, Z.; Wang, F.; et al. Enhancing Anti-PD-1/PD-L1 Immune Checkpoint Inhibitory Cancer Therapy by CD276-Targeted Photodynamic Ablation of Tumor Cells and Tumor Vasculature. Mol. Pharm. 2019, 16, 339-348.

95. Nagaya, T.; Friedman, J.; Maruoka, Y.; Ogata, F.; Okuyama, S.; Clavijo, P.E.; Choyke, P.L.; Allen, C.; Kobayashi, H. Host immunity following near-infrared photoimmunotherapy is enhanced with PD-1 checkpoint blockade to eradicate established antigenic tumors. Cancer Immunol. Res. 2019, 7, 401-413.

96. Monu, N.R.; Frey, A.B. Myeloid-derived suppressor cells and anti-tumor T cells: A complex relationship. Immunol. Invest. 2012.

97. Schmidt, A.; Oberle, N.; Krammer, P.H. Molecular mechanisms oftreg-mediatedt cell suppression. Front. Immunol. 2012.

98. Reginato, E.; Mroz, P.; Chung, H.; Kawakubo, M.; Wolf, P.; Hamblin, M.R. Photodynamic therapy plus regulatory T-cell depletion produces immunity against a mouse tumour that expresses a selfantigen. Br. J. Cancer 2013, 109, 2167-2174. 
99. Korbelik, M.; Banáth, J.; Zhang, W. Mreg activity in tumor response to photodynamic therapy and photodynamic therapy-generated cancer vaccines. Cancers (Basel). 2016.

100. Kumai, T.; Oikawa, K.; Aoki, N.; Kimura, S.; Harabuchi, Y.; Celis, E.; Kobayashi, H. Tumorderived TGF- $\beta$ and prostaglandin E2 attenuate anti-tumor immune responses in head and neck squamous cell carcinoma treated with EGFR inhibitor. J. Transl. Med. 2014.

101. Robbins, P.F.; El-Gamil, M.; Li, Y.F.; Kawakami, Y.; Loftus, D.; Appella, E.; Rosenberg, S.A. A mutated $\beta$-catenin gene encodes a melanoma-specific antigen recognized by tumor infiltrating lymphocytes. J. Exp. Med. 1996.

102. Boon, T.; Old, L. Cancer Tumor antigens. Curr. Opin. Immunol. 1997.

103. Wang, X.G.; Revskaya, E.; Bryan, R.A.; Strickler, H.D.; Burk, R.D.; Casadevall, A.; Dadachova, E. Treating cancer as an infectious disease-viral antigens as novel targets for treatment and potential prevention of tumors of viral etiology. PLoS One 2007.

104. Melief, C.J.M.; Van Hall, T.; Arens, R.; Ossendorp, F.; Van Der Burg, S.H. Therapeutic cancer vaccines. J. Clin. Invest. 2015.

105. Wculek, S.K.; Cueto, F.J.; Mujal, A.M.; Melero, I.; Krummel, M.F.; Sancho, D. Dendritic cells in cancer immunology and immunotherapy. Nat. Rev. Immunol. 2019.

106. Kushibiki, T.; Tajiri, T.; Tomioka, Y.; Awazu, K. Photodynamic therapy induces interleukin secretion from dendritic cells. Int. J. Clin. Exp. Med. 2010, 3, 110-114.

107. Zhang, F.; Zhu, Y.; Fan, G.; Hu, S. Photodynamic therapy reduces the inhibitory effect of osteosarcoma cells on dendritic cells by upregulating HSP70. Oncol. Lett. 2018.

108. Yang, W.; Zhu, G.; Wang, S.; Yu, G.; Yang, Z.; Lin, L.; Zhou, Z.; Liu, Y.; Dai, Y.; Zhang, F.; et al. In Situ Dendritic Cell Vaccine for Effective Cancer Immunotherapy. ACS Nano 2019.

109. Zheng, Y.; Yin, G.; Le, V.; Zhang, A.; Chen, S.; Liang, X.; Liu, J. Photodynamic-therapy Activates Immune Response by disrupting Immunity Homeostasis of Tumor Cells, which Generates Vaccine for Cancer Therapy. Int. J. Biol. Sci. 2016, 12, 120-132.

110. Jung, N.C.; Kim, H.J.; Kang, M.S.; Lee, J.H.; Song, J.Y.; Seo, H.G.; Bae, Y.S.; Lim, D.S. Photodynamic therapy-mediated DC immunotherapy is highly effective for the inhibition of established solid tumors. Cancer Lett. 2012.

111. Zhang, N.; Bai, S.; Cai, X.; Li, L. Inhibitory and immunological effects induced by the combination of photodynamic therapy and dendritic cells on mouse transplanted hepatoma. Photodiagnosis Photodyn. Ther. 2015, 8-11.

112. Kleinovink, J.W.; van Driel, P.B.; Snoeks, T.J.; Prokopi, N.; Fransen, M.F.; Cruz, L.J.; Mezzanotte, L.; Chan, a.; Lowik, C.W.; Ossendorp, F. Combination of Photodynamic Therapy and Specific Immunotherapy Efficiently Eradicates Established Tumors. Clin. Cancer Res. 2015, 1-11.

113. Zom, G.G.; Willems, M.M.J.H.P.; Khan, S.; Van Der Sluis, T.C.; Kleinovink, J.W.; Camps, M.G.M.; Van Der Marel, G.A.; Filippov, D. V.; Melief, C.J.M.; Ossendorp, F. Novel TLR2-binding adjuvant induces enhanced $\mathrm{T}$ cell responses and tumor eradication. J. Immunother. Cancer 2018.

114. Yang, G.; Xu, L.; Xu, J.; Zhang, R.; Song, G.; Chao, Y.; Feng, L.; Han, F.; Dong, Z.; Li, B.; et al. Smart Nanoreactors for $\mathrm{pH}-$ Responsive Tumor Homing, Mitochondria-Targeting, and Enhanced Photodynamic-Immunotherapy of Cancer. Nano Lett. 2018.

115. Kammerer, R.; Buchner, A.; Palluch, P.; Pongratz, T.; Oboukhovskij, K.; Beyer, W.; Johansson, A.; Stepp, H.; Baumgartner, R.; Zimmermann, W. Induction of immune mediators in glioma and prostate cancer cells by non-lethal photodynamic therapy. PLoS One 2011, 6 .

116. Brackett, C.M.; Owczarczak, B.; Ramsey, K.; Maier, P.G.; Gollnick, S.O. IL-6 potentiates tumor resistance to photodynamic therapy (PDT). Lasers Surg. Med. 2011, 43, 676-685.

117. Skivka, L.M.; Gorobets, O.B.; Kutsenok, V. V; Lozinsky, M.O.; Borisevich, A.N.; Fedorchuk, A.G.; Kholin, V. V; Gamaleya, N.F. 5-aminolevulinic acid mediated photodynamic therapy of Lewis lung carcinoma: a role of tumor infiltration with different cells of immune system. Exp. Oncol. 2004, 26, 312-5.

118. Shams, M.; Owczarczak, B.; Manderscheid-Kern, P.; Bellnier, D. a.; Gollnick, S.O. Development of photodynamic therapy regimens that control primary tumor growth and inhibit secondary disease. Cancer Immunol. Immunother. 2015, 64, 287-297. 
119. Sitnik, T.M.; Hampton, J. a; Henderson, B.W. Reduction of tumour oxygenation during and after photodynamic therapy in vivo: effects of fluence rate. Br. J. Cancer 1998, 77, 1386-94.

120. Henderson, B.W.; Busch, T.M.; Vaughan, L. a.; Frawley, N.P.; Babich, D.; Sosa, T. a.; Zollo, J.D.; Dee, A.S.; Cooper, M.T.; Bellnier, D. a.; et al. Photofrin photodynamic therapy can significantly deplete or preserve oxygenation in human basal cell carcinomas during treatment, depending on fluence rate. Cancer Res. 2000, 60, 525-529.

121. Henderson, B.W.; Gollnick, S.O.; Snyder, J.W.; Busch, T.M.; Kousis, P.C.; Cheney, R.T.; Morgan, J. Choice of Oxygen-Conserving Treatment Regimen Determines the Inflammatory Response and Outcome of Photodynamic Therapy of Tumors. Cancer Res. 2004, 64, 2120-2126.

122. De Vijlder, H.C.; Sterenborg, H.J.C.M.; Martino Neumann, H.A.; Robinson, D.J.; De Haas, E.R.M. Light fractionation significantly improves the response of superficial basal cell carcinoma to aminolaevulinic acid photodynamic therapy: Five-year follow-up of a randomized, prospective trial. Acta Derm. Venereol. 2012, 92, 641-647. 



\section{CHAPTER 7}

\section{The potential of nanobody-targeted photodynamic therapy to} trigger immune responses

Irati Beltrán Hernández ${ }^{1,2}$, Mathieu L. Angelier ${ }^{2}$, Tommaso Del Buono D’Ondes ${ }^{1}$, Alessia Di Maggio², Yingxin $\mathrm{Yu}^{2}$ and Sabrina Oliveira ${ }^{1,2}$

\footnotetext{
${ }^{1}$ Pharmaceutics, Department of Pharmaceutical Sciences, Faculty of Science, Utrecht University, Utrecht, the Netherlands

${ }^{2}$ Cell Biology, Neurobiology and Biophysics, Department of Biology, Faculty of Science, Utrecht University, Utrecht, the Netherlands
}

Cancers 2020, 12, 978 


\begin{abstract}
Nanobody-targeted photodynamic therapy (NB-PDT) has been recently developed as a more tumor-selective approach rather than conventional photodynamic therapy (PDT). NB-PDT uses nanobodies that bind to tumor cells with high affinity, to selectively deliver a photosensitizer, i.e., a chemical which becomes cytotoxic when excited with light of a particular wavelength. Conventional PDT has been reported to be able to induce immunogenic cell death, characterized by the exposure/release of damage-associated molecular patterns (DAMPs) from dying cells, which can lead to antitumor immunity. We explored this aspect in the context of NB-PDT, targeting the epidermal growth factor receptor (EGFR), using high and moderate EGFR-expressing cells. Here we report that, after NB-PDT, the cytoplasmic DAMP HSP70 was detected on the cell membrane of tumor cells and the nuclear DAMP HMGB1 was found in the cell cytoplasm. Furthermore, it was shown that NB-PDT induced the release of the DAMPs HSP70 and ATP, as well as the pro- inflammatory cytokines IL- $1 \beta$ and IL-6. Conditioned medium from high EGFR-expressing tumor cells treated with NB-PDT led to the maturation of human dendritic cells, as indicated by the upregulation of CD86 and MHC II on their cell surface, and the increased release of IL-12p40 and IL-1 $\beta$. Subsequently, these dendritic cells induced CD4+ T cell proliferation, accompanied by IFN $\gamma$ release. Altogether, the initial steps reported here point towards the potential of NB-PDT to stimulate the immune system, thus giving this selective-local therapy a systemic reach.
\end{abstract}




\section{Introduction}

Photodynamic therapy (PDT) was first approved in 1993 for the treatment of bladder cancer and, since then, its use has expanded to many other oncological indications, such as lung, brain, esophagus, skin, and head and neck cancer [1]. This treatment relies on the action of a photosensitizer (PS), i.e., a light activatable compound that accumulates into cells and becomes cytotoxic when excited with light of a particular wavelength. Upon PS activation, reactive oxygen species are generated that ultimately lead to cell death $[1,2]$. Besides direct cytotoxicity, damage to the tumor vasculature and the potential to stimulate an antitumor immune response have been reported [2-4]. This last aspect is of particular interest since a local treatment such as PDT, making use of locally applied light at the tumor site, could develop systemic effects via the activation of the immune system.

Despite being a selective and controllable treatment against tumor cells, therapies known to be considerably aggressive towards all tissues (i.e., chemo- and radiotherapy) are still the mainstream clinical practice, when it comes to combat cancer [5]. Some current barriers for the establishment of PDT in routine practice are the required technology, complex dosimetry, limited tissue penetration of light and/or PS, lack of tumor specificity and prolonged skin photosensitivity [1,5]. Efforts have been put into improving the selectivity of PDT towards the cancer cells by using antibodies, an approach which is now in phase II clinical trial (NCT02422979) [6]. Conjugation of a water-soluble PS (IRDye700DX) to an antibody allows the PS to be specifically delivered to cancer cells overexpressing a certain antigen on the cell membrane. In this manner, two levels of specificity are established: the delivery of PS to target-expressing cells and the local application of light at the tumor site.

Another strategy to render PDT more tumor-selective is nanobody-targeted PDT (NB-PDT), which also utilizes the near-infrared PS IRDye700DX. In this case, however, a nanobody is used to provide the first level of specificity $[7,8]$. NBs are the smallest binding domains found in nature $(\sim 15 \mathrm{kDa})$ and consist uniquely of the variable domain of heavy chain antibodies present in Camelids [9]. The use of a NB for PDT brings a series of advantages over antibody-targeted PDT. Their small size enables rapid tumor accumulation, with a homogeneous distribution, and a rapid clearance from circulation when unbound [9-11]. In the context of PDT, this allows the application of the light shortly after conjugate administration, ensures more extensive tumor damage, and reduces the chances of phototoxicity present in both PDT and antibody-targeted PDT protocols. In vitro, NB-PDT has been reported to be a very specific, selective, and potent approach to kill cancer cells expressing a variety of membrane receptors, such as epidermal growth factor receptor (EGFR) [7,8], human epidermal growth factor receptor 2 (HER2) [12], hepatocyte growth factor receptor (c-Met) [13], and G protein- coupled receptor (GPCR) [14]. Furthermore, NB-PDT has proven very effective in inducing tumor necrosis in an orthotopic mouse model of head and neck cancer expressing EGFR [7], and to induce significant tumor regression in an orthotopic mouse model of breast cancer expressing HER2 [12]. Additionally, similar to the observations with conventional PDT, damage to the tumor vasculature induced by NB-PDT has also been reported [15].

Subsequently, we aimed to explore the third component of responses triggered by PDT, i.e., whether NB-PDT can also trigger the immune system, possibly leading to an antitumor response, which would greatly add to the potential that NB-PDT has shown so far. This ability of PDT to stimulate immune cells has been linked to the fact that PDT is able to induce an immunogenic cell death (ICD). This type of cell death is accompanied by the release and exposure of several molecules from the dying cells, such as endogenous cytokines and damage-associated molecular patterns (DAMPs), which can be recognized 
by various activating receptors (e.g., pattern recognition receptors) on immune cells and, thereby, stimulate subsequent immune responses [16]. For instance, heat shock protein 70 (HSP70), high mobility group box 1 protein (HMGB1), calreticulin, and ATP can all act as DAMPs described to be exposed/released after PDT and associated with activation of innate immunity. These can trigger the local innate immune system (e.g., dendritic cells, DCs), thus paving the way towards the development of an adaptive memory immune response $[3,17]$, which is indispensable for preventing tumor recurrence and the formation of tumor metastasis in the long term. Importantly, the successful development of an anti-tumor response post-PDT relies largely on the initial induction of ICD [18].

In this study, we address the immunogenic potential of NB-PDT for the first time, from the initially induced tumor cell death mechanism, to the last step of the immune reaction involving activation of $\mathrm{T}$ cells. The EGFR-targeting NB-PS conjugate named 7D12-PS was employed for NB-PDT, a conjugate that has already shown its potency for the selective killing of EGFR-expressing cells [8]. Due to its slow internalization, after binding to EGFR, 7D12-PS is mostly present on the cell membrane when light is applied, thereby inducing membrane damage and subsequent cell death [8]. In vivo, this nanobody has been described to present rapid tumor accumulation and homogenous distribution $[10,11]$ and, upon illumination, induces extensive tumor damage, as well as vascular effects $[7,15]$. In order to take into account possible inter-tumor heterogeneity of target expression, two different tumor cell lines were included: the A431 cell line that overexpresses EGFR and the scc- U8 cell line expressing moderate EGFR levels, which more closely resemble the in vivo levels $[19,20]$. With this in vivo mirroring in mind, a highly cytotoxic NB-PDT (LD100) and a mild treatment (LD50) are also compared here, since uneven light penetration and tumor heterogeneity would likely lead to different degrees of cytotoxicity in vivo. Our results show that NB-PDT induces rapid necrosis accompanied by exposure/release of major DAMPs and cytokines from the dying cells. This release of immunogenic factors leads to the maturation of DCs which subsequently activate CD4+ T cells, thereby demonstrating the immunogenicity triggered by NB-PDT.

\section{Materials and Methods}

\section{Cell Lines and Nanobody-Photosensitizer Conjugates}

The human epidermoid carcinoma cell line A431 was purchased from ATCC, and the human head and neck carcinoma cell line scc-U8 was kindly provided by Dr. Robinson (Erasmus MC, Rotterdam, The Netherlands). A431 cells are used as a reference cell line which overexpresses EGFR $\left(0.5-3.5 \times 10^{6}\right.$ receptors per cell), while scc-U8 cells are a more representative tumor cell line with $36 \%$ EGFR expression relative to A431 cells $[19,49]$. Both cell lines were cultured in Dulbecco's Modified Eagle's Medium (DMEM) (Lonza) supplemented with 10\% foetal bovine serum (FBS) (Sigma-Aldrich), $100 \mathrm{U} / \mathrm{mL}$ penicillin and $100 \mu \mathrm{g} / \mathrm{mL}$ streptomycin (Sigma-Aldrich). Cells were cultured at $37{ }^{\circ} \mathrm{C}$ and $5 \% \mathrm{CO}_{2}$.

The EGFR-targeting monomeric NB 7D12 and the biparatopic NB 7D12-9G8, as well as their conjugation to the PS IRDye700DX N-hydroxysuccinimidine ester (LICOR), have been previously described [7].

\section{Nanobody-Targeted Photodynamic Therapy}

A431 or scc-U8 cells were seeded in 96-well plates (10.000 and 15.000 cells/well, respectively) one day before the assay. The next day, cells were washed once with PDT 
medium, i.e., DMEM without phenol red (Lonza) supplemented with $10 \%$ FBS and antibiotics, and the concentration of conjugate to achieve $50 \%$ (LD50) or $100 \%$ (LD100) of cell death was added ( 1 and $25 \mathrm{nM}$ for A431 cells, 10 and $100 \mathrm{nM}$ for scc-U8 cells) (see Figure S1). Cells were incubated with the conjugates for $30 \mathrm{~min}$ at $37^{\circ} \mathrm{C}$. Thereafter, cells were washed twice with PDT medium and illuminated with $5 \mathrm{~mW} / \mathrm{cm} 2$ for $33 \mathrm{~min}$ in the case of A431 cells, or $7 \mathrm{~mW} / \mathrm{cm}^{2}$ for $59 \mathrm{~min}$ in the case of scc-U8 cells (equal to 10 and $25 \mathrm{~J} / \mathrm{cm}^{2}$, respectively). Fluence rate was measured with an Orion/PD optometer (Ophir Optronics) and light applied using a $690 \mathrm{~nm}$ laser (Modulight, ML7700). Cells were always seeded in two separate 96-well plates: one plate receiving light including conditions without conjugate (only light control), and LD50 and LD100 concentrations (NB-PDT); and another plate not illuminated and featuring the controls of LD100 (only conjugate control) and untreated cells.

\section{Mechanism of Cell Death}

Right after NB-PDT, CellEvent caspase 3/7 green detection reagent (caspase 3/7) (Invitrogen) and propidium iodide (PI) (Invitrogen) were added to the cells in a final dilution of 1:10 and 1:1000, respectively. Well plates were placed back in the incubator for $2 \mathrm{~h}$ and, hereafter, cells were imaged with an EVOS microscope (Thermo Fisher Scientific) using transmitted light, the GFP light cube for caspase 3/7, and the RFP light cube for PI (20x objective). Plates were returned to the incubator and imaged again $18 \mathrm{~h}$ after NB-PDT.

\section{Cellular Localization of HSP70 and HMGB1 on Tumor Cells}

NB-PDT was performed as described above, with the only difference being that cells were seeded on 16 wells Lab-Tek Chamber Slides (Thermo Fisher Scientific, 178599). For HSP70 detection, chamber slides were returned to the incubator after NB-PDT for 4 $\mathrm{h}$, followed by $10 \mathrm{~min}$ at room temperature and $10 \mathrm{~min}$ at $4{ }^{\circ} \mathrm{C}$. Cells were washed with medium (DMEM without phenol red supplemented with $25 \mathrm{mM}$ HEPES and 2\% BSA, pH 7.2) and the primary antibody Mouse anti-HSP70 (Thermo Fisher Scientific, MA3009) was added for $1 \mathrm{~h}$ at $4{ }^{\circ} \mathrm{C}(1: 100$ in medium). Cells were fixed with $4 \%$ PFA (Merck) for $15 \mathrm{~min}$ at room temperature and the secondary antibody Goat anti-Mouse Alexa555 (Molecular probes, A21424) was added for $1 \mathrm{~h}$ at room temperature (1:200 in PBS $+2 \%$ BSA). Cells were stained with DAPI (Roche) and imaged with a Confocal Laser Scanning Microscope (Carl Zeiss Microscopy GmbH, LSM700) using a plan-apochromat 63x/1.40 Oil DIC objective.

For HMGB1 detection, chamber slides were returned to the incubator after NBPDT for $4 \mathrm{~h}$, followed by $10 \mathrm{~min}$ at room temperature. Cells were fixed with $4 \%$ PFA and permeabilized with $0.2 \%$ Triton X-100 (Sigma-Aldrich) for $15 \mathrm{~min}$ at room temperature. The primary antibody Rabbit anti- HMGB1 (Invitrogen, PA1-16926) was added for $1 \mathrm{~h}$ at room temperature (1:50 in in PBS $+2 \%$ BSA), followed by staining with the secondary antibody Donkey anti-Rabbit Alexa488 (Invitrogen, A21206) for $1 \mathrm{~h}$ at room temperature (1:500 in PBS $+2 \%$ BSA).

\section{Detection of ATP in the Supernatant of Tumor Cells}

NB-PDT was performed, plates were placed back in the incubator and supernatants were collected $4 \mathrm{~h}$ later. Cell debris was removed by spinning down, supernatants were added to a white 96-well plate (Greiner Bio-One) and mixed with CellTiter-Glo 3D (Promega, G9681) in a 1:1 ratio. Plates were incubated for $5 \mathrm{~min}$ on a horizontal orbital microplate shaker followed by an incubation of $25 \mathrm{~min}$ at room temperature, protected from light. 
Luminescence was measured with a GloMax 96 ${ }^{\mathrm{TM}}$ microplate luminometer (Promega). Assay performance was verified each time by including ATP standards (Promega).

\section{Detection of HSP70 in the Supernatant of Tumor Cells}

NB-PDT was performed as described above, plates were placed back in the incubator and the supernatants were collected $24 \mathrm{~h}$ later. Cell debris was removed and a Human HSP70 ELISA kit (Invitrogen, BMS2087) was used with the supernatants, according to the manufacturer's protocol.

\section{Detection of IL-1 $\beta$, IL-6, and IL-8 in the Supernatant of Tumor Cells}

A431 cells or scc-U8 cells were seeded on $6 \mathrm{~cm}$ petri dishes $(650.000$ and 700.000 cells/dish, respectively). The next day, NB-PDT was performed as described above and, afterwards, dishes were placed back in the incubator for $24 \mathrm{~h}$. Supernatants were collected and concentrated using 3K Amicon Ultra Centrifugal Filters (Merck). IL-6, IL-8, and IL$1 \beta$ were then detected in the concentrated supernatants using a Human Magnetic Luminex Assay (R\&D Systems), following the protocol of the manufacturer.

\section{Generation, Stimulation and Analysis of Dendritic Cells Maturation}

Human peripheral blood mononuclear cells (PBMCs) were isolated from buffy coats of healthy donors by means of density gradient centrifugation using LeucoSep tubes (Greiner Bio-One), followed by CD14+ monocyte isolation using magnetic negative selection with a Monocyte Isolation Kit (Miltenyi Biotec). Monocytes were cultured for 7 days in Roswell Park Memorial Institute (RPMI) 1640 with L-glutamine (Thermo Fisher Scientific) supplemented with 10\% fetal calf serum (Bodinco), $100 \mathrm{U} / \mathrm{mL}$ penicillin/streptomycin (Thermo Fisher Scientific), $100 \mathrm{ng} / \mathrm{mL}$ IL-4 (ProSpec), and 60 $\mathrm{ng} / \mathrm{mL}$ GM-CSF (ProSpec). Immature moDCs were harvested at day 8 and seeded into a 96-well plate at a density of $1 \times 105$ cells/well in culture medium without IL-4 and GMCSF. Immature moDCs were either left unstimulated, stimulated with $100 \mathrm{ng} / \mathrm{mL}$ LPS (InvivoGen, E. coli 055:B5) or with tumor conditioned media from different NB-PDT conditions. On average, $1 \times 105$ moDCs were incubated with supernatant of $3 \times 104$ tumor cells. After $24 \mathrm{~h}$ of stimulation, moDCs were collected, stained for surface markers and analyzed by flow cytometry using a FACS Canto II (BD Biosciences). Cells were stained with antibodies against the following markers: CD11c (PerCP- e710), CD14 (APC), CD86 (PE-Cy7) and MHCII (PE) (eBioscience), as well as the viability dye YOPRO1 (Thermo Fisher Scientific). Expression of CD86 on moDCs was quantified as \% of positive cells, due to its bimodal distribution, while mean fluorescence intensity (MFI) was used to denote MHCII expression. Furthermore, supernatants were collected and stored at $-20^{\circ} \mathrm{C}$ to later on measure IL- $12 \mathrm{p} 40$, IL- $1 \beta$ and IL-10 levels by Luminex (R\&D Systems), according to the manufacturer's protocols.

\section{Generation and Analysis of CD4+ T Cells Activation}

Human PBMCs were isolated from buffy coats of healthy donors by means of density gradient centrifugation using LeucoSep tubes (Greiner), followed by CD4+ cells isolation using magnetic negative selection with a Naïve CD4+ T cell isolation kit II human (Miltenyi Biotec, 130-094-131). Naïve CD4+ cells were labeled with the proliferation dye CFSE (Thermo Fisher Scientific, C34570) and co- cultured with allogeneic moDCs (stimulated as described above) at DC:T cell ratio of 1:10 in Iscove's Modified Dulbecco's Medium (IMDM) with glutamax (Gibco), supplemented with 10\% fetal calf serum, $100 \mathrm{U} / \mathrm{mL}$ penicillin/streptomycin, $20 \mu \mathrm{g} / \mathrm{mL}$ apo-transferrine and $50 \mu \mathrm{M} \beta$ - 
mercaptoethanol. After 6 days of co-incubation, cells were collected and stained with antibodies anti-CD4-PerCP-Cy5.5 (eBioscience, 45-0048-42) and anti-CD3-APC (BioLegend, 300412). Samples were analyzed by flow cytometry using a FACS Canto II (BD Biosciences) and the proliferating fraction identified based on the CFSE-negative population from samples with CFSE-labeled CD4+ $\mathrm{T}$ cells alone. Furthermore, the supernatants derived from the co-incubation were collected and stored at $-20{ }^{\circ} \mathrm{C}$ to measure later IFN $\gamma$ levels by ELISA (Invitrogen), according to the manufacturer's protocol.

\section{Statistical Analysis}

Data were analyzed with GraphPad Prism 8 software using one-way ANOVA with a Tukey post-hoc test to compare between conditions. Statistical significance was displayed as $* \mathrm{p} \leq 0.05, * * \mathrm{p} \leq 0.01$, and $* * * \mathrm{p} \leq 0.001$.

\section{Results}

\section{Necrosis Is the Main Cell Death Mechanism Induced by NB-PDT}

Taking into account possible variations in EGFR expression and in light penetration, the type of cell death induced by NB-PDT was investigated after mild (LD50) and highly cytotoxic (LD100) NB-PDT, on high and moderate EGFR-expressing cells, i.e., A431 and scc-U8 cells, respectively. An overview of the LD50 and LD100 used in this study for each cell line is depicted on Figure S1. The cell death mechanism was studied 2 and $18 \mathrm{~h}$ after treatment, by means of fluorescent dyes denoting apoptosis or necrosis. Necrosis was the main cell death mechanism triggered by NB-PDT on A431 cells (Figure 1a). Cell morphology resembled the positive control for necrosis, reflecting the extensive damage that this conjugate induces on the cell membrane. Morphological changes on the cells $2 \mathrm{~h}$ after treatment, i.e., rounded up cells, indicate that cells are rapidly affected by NB-PDT. Contrary to A431 cells, scc-U8 cells showed only slight morphological changes shortly after treatment, while after $18 \mathrm{~h}$ a mixture of both necrotic and apoptotic cells could be detected under the highly cytotoxic condition (Figure 1b). No cell death signal was detected upon the use of the individual components of NB-PDT, indicating the lack of toxicity when using the conjugate or light alone (Figure S2a).

\section{Cellular Localization of HSP70 and HMGB1 Change on Tumor Cells Treated with NB-PDT}

Changes in the localization of several DAMPs within tumor cells were investigated $4 \mathrm{~h}$ after treatment with NB-PDT. This early time point was chosen to allow visualization of not yet severely damaged cells and facilitate detection of these DAMPs in the different cell compartments. Here, the DAMPs HSP70 (a cytoplasmic chaperone) and HMGB1 (a nuclear chromosomal protein) were considered. An increase of HSP70 on the cell membrane was observed after mild NB-PDT in comparison to untreated cells (Figure $2 \mathrm{a}, \mathrm{b}$ ) and controls of only light or conjugate (Figure S2b). On the other hand, HSP70 was detected in the cytoplasm and nucleus of cells after the highly cytotoxic treatment in the case of A431 cells. This is in line with the extensive damage imposed on the cell membrane with this NB-PDT condition, resulting in a permeabilized membrane allowing intracellular detection of HSP70. Overall, the membrane staining of HSP70 was more evident on A431 cells than on scc-U8 cells and no extensive membrane damage was yet 
observed on scc-U8 cells after the highly cytotoxic treatment in the time frame of this experiment.

HMGB1 was predominantly present in the nucleus of untreated A431 and scc-U8 cells (Figure 2c,d), as well as control cells exposed to only light or conjugate (Figure S2c). After mild and highly cytotoxic NB-PDT, HMGB1 was detected in both nucleus and cytoplasm of the cells. Of note, HMGB1 was found to be almost completely excluded from the nucleus in a large number of A431 cells under the highly cytotoxic condition, again indicative of the substantial damage inflicted by NB-PDT to this cell line at early time points. Overall, both A431 and scc-U8 cells depicted a similar behavior regarding the changes in localization of both DAMPs.
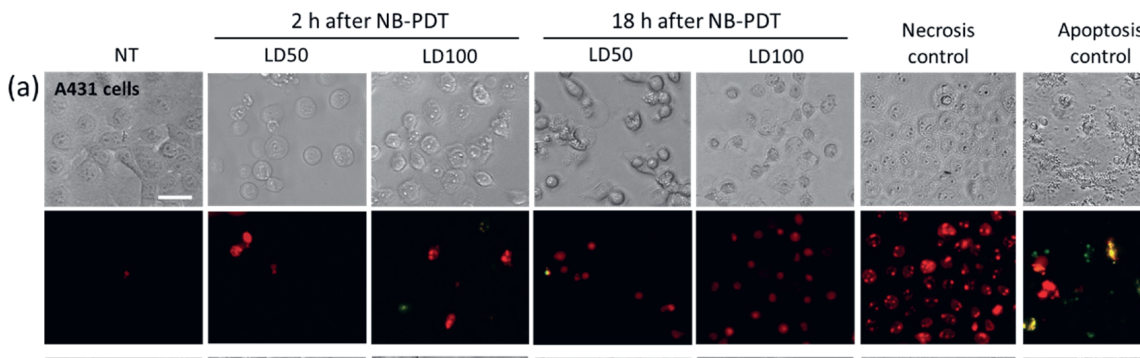

(b)
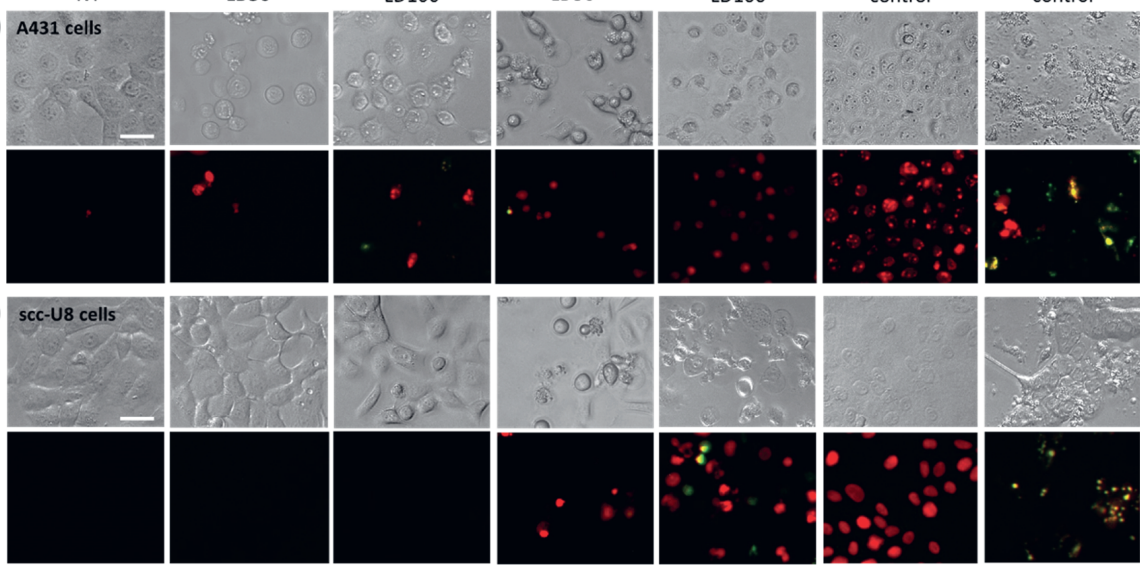

Figure 1 Cell death mechanism induced by nanobody-targeted photodynamic therapy (NB-PDT). Tumor cells were left untreated (NT) or treated with NB-PDT using 7D12-PS (LD50 or LD100) and stained with propidium iodide (PI) for necrotic cells (red) and caspase 3/7 for apoptotic cells (green), controls for necrosis and apoptosis were included. Microscopy images of (a) A431 cells and (b) scc-U8 cells were taken 2 and $18 \mathrm{~h}$ after NB-PDT. Top panels depict the transmitted light image and bottom panels the merged images of necrotic and apoptotic cells. Scale bar, $20 \mu \mathrm{m}$.

\section{NB-PDT Induces ATP and HSP70 Release from Treated Tumor Cells}

For both cell lines, a higher amount of ATP was detected in the supernatant of tumor cells $4 \mathrm{~h}$ after NB-PDT, under both mild and highly cytotoxic conditions (Figure 3a,b). A431 cells treated with NB-PDT showed higher release of ATP when compared with scc-U8 cells. Similarly, high concentrations of HSP70 were detected in the supernatant of treated tumor cells (Figure 3c,d). Nonetheless, in this case, the HSP70 concentration in the supernatants was comparable between cell lines. For both DAMPs, release was always more pronounced when cells were treated with the highly cytotoxic NB-PDT. 
(a)

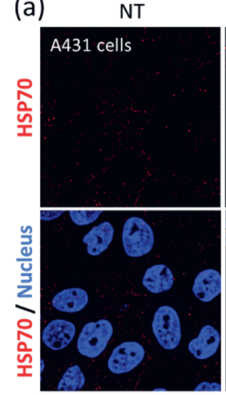

(c)

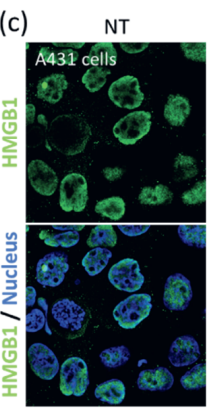

LD50

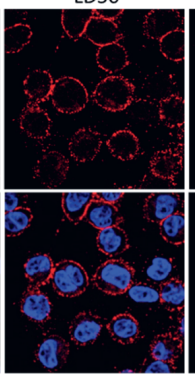

LD50

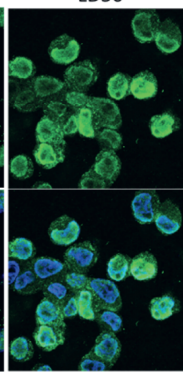

LD100

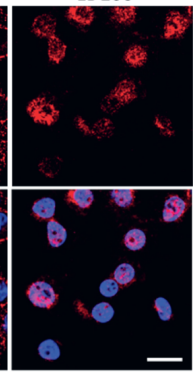

LD100

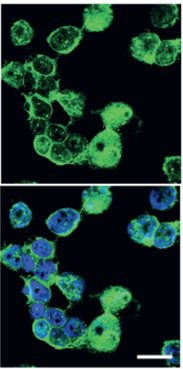

(b)

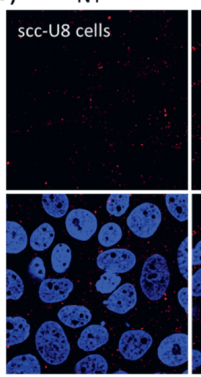

(d)

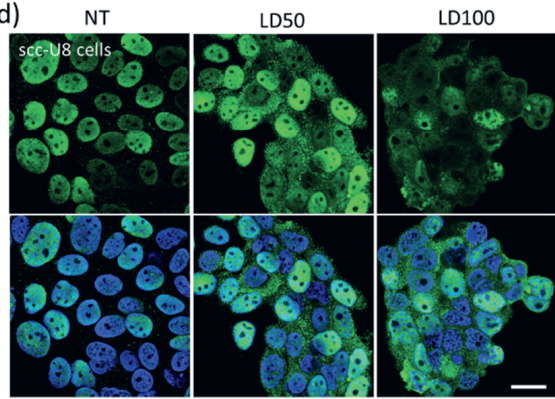

Figure 2 Cellular localization of heat shock protein 70 (HSP70) and high mobility group box 1 protein (HMGB1) on tumor cells treated with NB-PDT. Tumor cells were left untreated (NT) or treated with NB-PDT using 7D12-PS (LD50 or LD100), and $4 \mathrm{~h}$ later stained for HSP70 or HMGB1. Staining of HSP70 (red) was performed on non-permeabilized (a) A431 cells and (b) scc-U8 cells. Intracellular staining of HMGB1 (green) was performed on (c) A431 cells and (d) scc-U8 cells. Cell nuclei were additionally stained with DAPI (blue). Top panels depict only the damage-associated molecular pattern (DAMP) signal, while merged images are shown on the bottom panels. Scale bar, $20 \mu \mathrm{m}$.

(a)

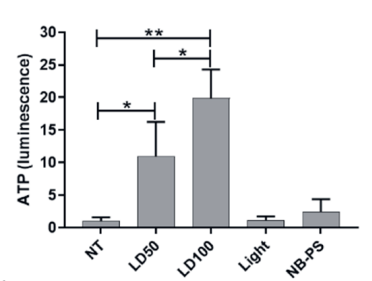

(c)

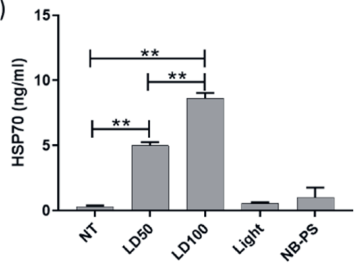

(b)

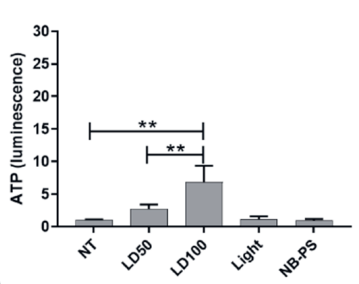

(d)

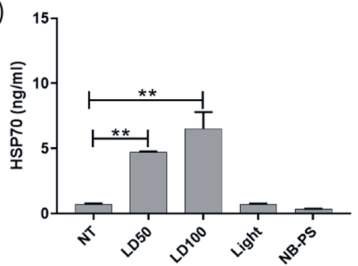

Figure 3 Quantification of ATP and HSP70 release from tumor cells after NB-PDT. ATP in the supernatant was detected $4 \mathrm{~h}$ after NB-PDT via a luminescence assay and graphs show luminescence values relative to untreated cells for (a) A431 cells and (b) scc-U8 cells. Additionally, released HSP70 was detected $24 \mathrm{~h}$ after treatment using ELISA on (c) A431 cells 
and (d) scc-U8 cells. NT, untreated; LD50, mild cytotoxic NB-PDT; LD100, highly cytotoxic NB-PDT; Light, only light control; NB-PS, only nanobody-photosensitizer (NB-PS) conjugate control. Significance is displayed as $* \mathrm{p} \leq 0.05$ and $* * \mathrm{p} \leq 0.01$.

\section{Cytokine Levels Released by Tumor Cells Are Altered after NB-PDT}

Release of particular cytokines from tumor cells was investigated after NB-PDT. High concentrations of the proinflammatory cytokines IL-1 $\beta$ (Figure 4a) and IL-6 (Figure 4b) were quantified in the supernatants of A431 cells treated with the highly cytotoxic NBPDT. Changes regarding the levels of these cytokines were less pronounced on the moderate-EGFR expressing scc- U8 cells (Figure 4d,e), but similar trends were detected. Furthermore, both tumor cell lines secreted considerable amounts of IL-8, which were substantially reduced after both mild and highly cytotoxic NB-PDT (Figure 4c,f).
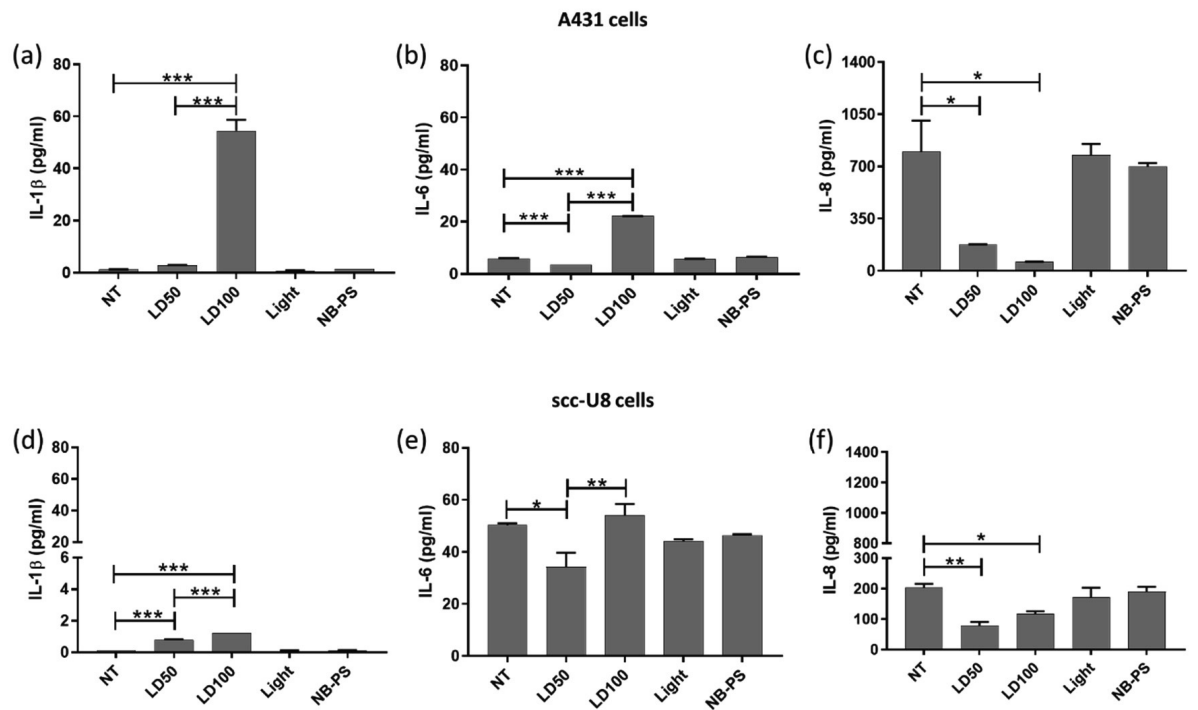

Figure 4 Quantification of IL-1 $\beta$, IL-6 and IL-8 release by tumor cells treated with NB-PDT. A431 or scc-U8 cells were treated with NB-PDT and the concentration of several cytokines in the supernatant was quantified $24 \mathrm{~h}$ later. Graphs display the quantification of IL-1 $\beta$, IL-6 and IL-8 on A431 cells ( $a, b$, and c, respectively) and on scc-U8 cells (d, e, and f, respectively). NT, untreated; LD50, mild cytotoxic NB-PDT; LD100, highly cytotoxic NB-PDT; Light, only light control; NB-PS, only NB-PS conjugate control. Significance is displayed as $* \mathrm{p} \leq 0.05, * * \mathrm{p} \leq$ 0.01 and $* * * \mathrm{p} \leq 0.001$.

\section{Maturation of Dendritic Cells Is Induced by NB-PDT Treated Tumor Supernatants}

Monocyte-derived DCs (moDCs) were incubated with the conditioned medium of tumor cells treated with NB-PDT and the expression of two maturation markers, MHCII (an antigen presenting molecule) and CD86 (a costimulatory molecule), on the surface of moDCs was evaluated. Lipopolysaccharide (LPS) stimulation was used as a positive control. Subsequently, increase of the CD86+ population was detected only when moDCs were incubated with LPS or conditioned medium of cells treated with highly cytotoxic NB-PDT (Figure 5a,b). All the other groups, including mild NB-PDT and controls of the single components of the treatment, failed to induce significant upregulation of this 
maturation marker. The same trend was observed for the upregulation of MHCII on moDCs, although significance was affected by the intrinsic differences between donors.

Besides phenotypic maturation, further activation of moDCs was investigated by measuring their release of IL-12, IL-1 $\beta$, and IL-10 after incubation with NB-PDT treated tumor supernatant. First, IL12-p70 detection in the supernatant of moDCs was attempted since this is the prime cytokine released by DCs to activate T cells. Nonetheless, results were negative due to the detection limit of the assay employed (not shown). On the other hand, a trend towards increased release of other main cytokines, i.e., IL-12p40 and IL$1 \beta$, was detected in moDCs incubated with tumor supernatant from the highly cytotoxic condition (Figure 5c,d), which can be associated with an immunostimulatory profile. Release of IL-10, an anti-inflammatory cytokine, was also found to be elevated in these moDCs (Figure 5e). From the five different donors from which moDCs were isolated, only the moDCs of one of these donors (green circles in Figure 5) did not respond to NBPDT in terms of phenotype maturation and cytokine release.

\section{Dendritic Cells Exhibit a Stronger Ability to Induce CD4+ T Cell Activation after Stimulation with NB-PDT Treated Tumor Supernatants}

After detecting increased expression of MHCII and CD86 on the surface of moDCs, we speculated that these stimulated moDCs, upon crosslinking, would increase CD4+ T cell activation. To prove this, stimulated moDCs were cocultured with allogeneic naïve CD4+ $\mathrm{T}$ cells and, after a 6- day coculture, the activation of $\mathrm{T}$ cell was investigated, which involves $\mathrm{T}$ cell proliferation accompanied by IFN $\gamma$ production. In this context, moDCs stimulated with supernatant of tumor cells treated with highly cytotoxic NB-PDT were able to enhance the proliferation (Figure 6a) and IFN $\gamma$ production (Figure $6 \mathrm{~b}$ ) of CD4+ T cells, compared to untreated moDCs. LPS treated moDCs served as positive control. On the contrary, no T cell activation was detected for any other NB-PDT condition or control.

(a)

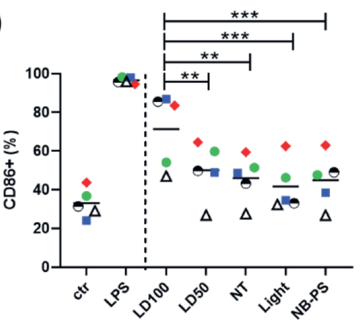

(c)

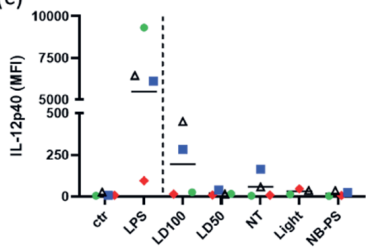

(d)

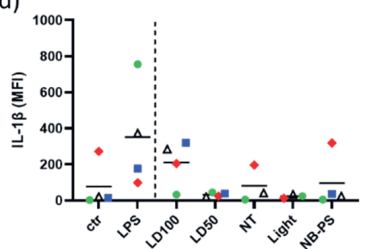

(b)

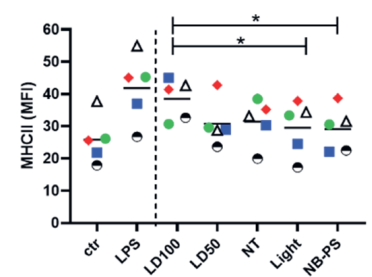

(e)

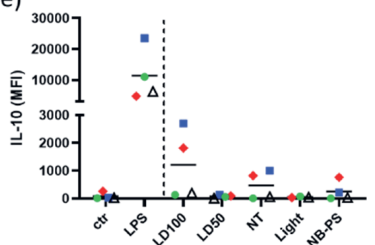

Figure 5 Phenotypic maturation and cytokine release of monocyte-derived dendritic cells (moDCs) incubated with supernatant of NB-PDT treated tumor cells. A431 cells were treated with NB-PDT, the supernatant was collected $24 \mathrm{~h}$ later and incubated with immature moDCs for another $24 \mathrm{~h}$. Surface marker expression on moDCs was measured with flow cytometry, and cytokine release was assessed by Luminex. (a) Percentage of CD86 positive moDCs. (b) Median fluorescence intensity (MFI) corresponding to MHCII surface expression on moDCs. Each moDC 
donor $(\mathrm{n}=5)$ is represented by a different symbol and color. ctr, unstimulated DCs; lipopolysaccharide (LPS), LPS-stimulated DCs; NT, untreated tumor cells; LD50, mild cytotoxic NB-PDT; LD100, highly cytotoxic NB-PDT; Light, only light control; NB-PS, only NB-PS conjugate control. Significance is displayed as $* \mathrm{p} \leq 0.05, * * \mathrm{p} \leq 0.01$, and $* * * \mathrm{p} \leq 0.001$. C-E, MFI corresponding to the release by moDCs of (c) IL-12p40, (d) IL-1 $\beta$ and (e) IL-10 ( $n=4)$. No statistical significance was found between groups due to the intrinsic differences between donors.

(a)

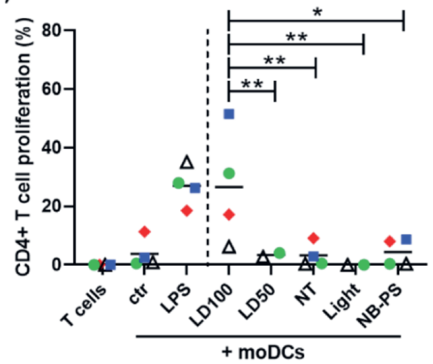

(b)

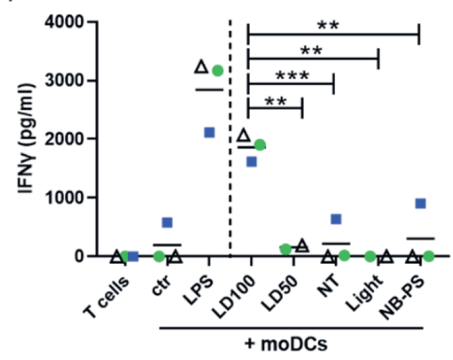

Figure 6 Enhanced proliferation and IFN $\gamma$ release of CD4+ T cells induced by moDCs stimulated with supernatant of NB-PDT treated tumor cells. A431 cells were treated with NB-PDT, the supernatant was collected $24 \mathrm{~h}$ later and incubated with immature moDCs for another $24 \mathrm{~h}$. moDCs were then co- incubated with allogeneic CFSE-labeled CD4+ T cells in a 1:10 ratio. After 6 days, CD4+ T cell proliferation was measured with flow cytometry and IFN $\gamma$ release was assessed by ELISA. (a) Percentage of CD4+ T cells with weak CFSE signal, thus proliferating cells $(n=4)$. (b) Quantification of released IFN $\gamma$ by CD4+ T cells $(n=3)$. Each combination of allogeneic donors is represented by a different symbol and color. ctr, unstimulated DCs; LPS, LPS-stimulated DCs; NT, untreated tumor cells; LD50, mild cytotoxic NB-PDT; LD100, highly cytotoxic NB-PDT; Light, only light control; NB-PS, only NB-PS conjugate control. Significance is displayed as $* \mathrm{p} \leq 0.05,{ }^{* *} \mathrm{p} \leq 0.01$ and ${ }^{* * *} \mathrm{p} \leq 0.001$.

\section{Discussion}

NB-PDT has been developed as a more tumor-selective alternative to PDT and has already shown great potential in vitro and in preclinical models $[7,8,13,14]$. NB-PDT allows light application shortly after PS administration, ensures extensive tumor damage, and is expected to reduce the post- treatment phototoxicity associated with some PDT protocols. Both NB-PDT and its conventional counterpart initially cause extensive damage to the primary tumor and to the tumor vasculature $[2,15]$. In addition, the conventional approach has been described to also stimulate an antitumor immunity $[3,4]$. Consequently, this local treatment can induce a systemic effect, a feature that is highly desirable for protection against metastasis and recurrences. In this study, for the first time, the immunomodulatory potential of NB-PDT is addressed. We describe the first steps regarding this aspect: in vitro NB-PDT induces a large extent of necrosis, leads to changes in cellular localization and release of well-known DAMPs and proinflammatory cytokines from tumor cells, and ultimately induces the maturation of moDCs and subsequent $\mathrm{CD} 4+\mathrm{T}$ cell activation.

It has been firmly established that PDT can be a potent inducer of ICD, which together with the triggered initial local inflammation, can promote the development of an adaptive immune response against the tumor [16]. Nonetheless, the development of such a response is not always successful since it is ultimately influenced by the type of PS and its cellular localization, tumor type and stage, and illumination protocol, among other 
factors [21]. To cover some of these aspects, we employed: (1) two tumor cell lines expressing high (A431 cells) and moderate (scc-U8 cells) EGFR levels, and (2) two concentrations of the conjugate 7D12-PS to yield mild (LD50) and highly cytotoxic (LD100) NB-PDT. In our previous studies, an additional NB-PS was employed, i.e., 7D12-9G8-PS. This nanobody has been described to lead to faster internalization of EGFR [22] and consequently to a more pronounced delivery of PS intracellularly [8], altogether leading to more toxicity in vitro (lower LD50 than 7D12-PS). However, in the context of this study, no significant differences were observed compared to 7D12-PS (Figure S4). Together with the fact that the monovalent nanobody format is expected to distribute more homogenously than a bivalent format [11], this study was focused on one conjugate only, i.e., 7D12-PS.

Conventional PDT mostly uses hydrophobic PSs which are internalized by the cells and mainly end up in the mitochondria, endoplasmic reticulum, or lysosomes. Consequently, the main cell death mechanism triggered by most PSs is apoptosis due to initial damage to these organelles [5]. We showed that, in the case of NB-PDT using 7D12-PS, necrosis is the main cause of cell death (Figure 1). This is because the conjugate is mostly present on the cell membrane at the time of the light application, as previously reported in [8], leading to its substantial damage. As a result of the loss of plasma membrane integrity, ATP depletion can rapidly occur, which directs the death program towards necrosis [23,24]. Likewise, extensive cell membrane damage has been reported for EGFR- targeted PDT using antibodies [25]. On the other hand, some apoptotic sccU8 cells were observed when treated with 7D12-PS, pointing towards the idea that lower membrane EGFR levels equals less abrupt damage on the cell membrane, thus giving the cell time to respond in other ways (e.g., apoptosis). Interestingly, the use of 7D12-9G8PS, which internalizes faster, led to cell death in a more rapid fashion than the monomeric counterpart (Figure S3a), which might be explained by a combination of higher avidity and internalization capacity. Besides necrosis, some degree of apoptosis was triggered by this conjugate, suggesting a similar organelle damage to that of conventional PSs when the NB-PS is internalized. Despite these differences in the induced mechanism of cell death, this did not seem to have a significant effect on the subsequent release of DAMPs (Figure S3b,c).

Overall, it seems that for NB-PDT the amount of NB-PS conjugate on the membrane is decisive for the induction of rapid necrosis, and we showed this can vary depending on target expression, conjugate concentration or internalizing rate of the conjugate. There is so far not a consensus on whether PDT-induced necrosis or apoptosis is more suitable to trigger an antitumor immune response, although some advocate the latter [26]. Nevertheless, it is likely that in the clinical setting both types of cell death will simultaneously occur, and both can contribute to the successful activation of immune cells due to the release and exposure of DAMPs and other immunogenic molecules from the dying cells [27].

This exposure of immunogenic molecules after NB-PDT was the next event explored in this study. Conventional PDT is described to induce exposure of HSP70, among other chaperones, on the membrane of tumor cells [28-31]. Membrane exposure of HSP70 was indeed observed on A431 and scc-U8 cells treated with mild NB-PDT, although it was more evident on the high EGFR-expressing cells at the early timepoint studied (Figure 2a,b). When HSP70 is located on the cell membrane, it displays unique functions since it can be recognized by antigen presenting cells and it aids in the crosspresentation of tumor antigens [32]. Hence, mild NB-PDT seems to provide a setting where these mechanisms can take place, while the highly cytotoxic condition relies mostly on a substantial release of DAMPs. Another DAMP, HMGB1, has been described 
to change its cellular localization after conventional PDT, from nuclear to cytoplasmic, being subsequently released $[28,30,33]$. The same changes in the localization of HMGB1 were observed here on both A431 and scc-U8 cells treated with NB-PDT (Figure 2c,d). In our case, the highly cytotoxic condition not only induced translocation of this DAMP to the cytoplasm, but it was also excluded from the nucleus in some A431 cells, as also described by other groups using conventional PDT [33].

The release from tumor cells of two DAMPS (i.e., HSP70 and ATP) was also addressed, knowing that both can determine the immunogenicity of the dying cells [27] and their release has been described after conventional PDT [28-30,34]. HSP70 and ATP were indeed released from both A431 cells (treated with either 7D12-PS or 7D12-9G8PS) and scc-U8 cells (treated with 7D12-PS) (Figure 3 and Figure S3b,c). In agreement with the extent of induced cell death, the highly cytotoxic NB-PDT always led to the largest release of these DAMPs, followed by the mild treatment, suggesting passive release of DAMPs from the necrotic cells. Although differences were observed in confocal images taken from A431 and scc-U8 cells (Figure 2a,b), the HSP70 concentration in the supernatants was comparable between cell lines. This can be explained by the fact that HSP70 release was quantified $24 \mathrm{~h}$ after treatment, when we expect complete effect of the LD50 and LD100 treatment and no differences between cell lines due to the less extensive damage on scc-U8 early after NB-PDT. Concerning the biparatopic conjugate, this did not yield higher amounts of released ATP than 7D12- PS (measured $4 \mathrm{~h}$ after NB-PDT), while the extent of cell death is considerably larger, shortly after treatment. This can be related to the induction of a mix of apoptosis and necrosis by 7D12-9G8-PS, where ATP is being utilized to execute the programmed cell death mechanism instead of being released. The release of HMGB1 was not investigated in our study, but its change in cellular localization (Figure 2c,d) suggests its release will eventually take place, where it can regulate antigen processing and presentation by DCs [32]. Importantly, one of the hallmarks of ICD, together with HMGB1 and ATP release, is calreticulin surface exposure. Our attempts to explore this event under the microscope have thus far not been successful. Antibody-targeted PDT induces only minimal calreticulin exposure compared to HSP70 or HSP90, as measured by flow cytometry, but is still able to trigger potent ICD [25]. Flow cytometry is a more sensitive technique than fluorescence microscopy, able to detect these slight changes. We believe the use of more sensitive and quantitative techniques would further support the potential of NB-PDT to induce ICD.

Besides DAMPs, cytokines can also be released by dying tumor cells. In this study, we were able to detect an increased release of the proinflammatory cytokines IL-1 $\beta$ and IL-6 from tumor cells treated with the highly cytotoxic NB-PDT (Figure 4). At the same time, IL-8 secretion was considerably decreased after NB-PDT. In this case, IL-8 might be having a protumoral function on the tumor cells, as suggested in [35], since high basal levels of released IL-8 are detected from untreated cells. Others have described modulation of cytokine production of IL- $1 \alpha$, IL-1 $\beta$, IL-2, IL-4, IL-6, IL-8, IL-10, IL-17A, TNF- $\alpha$, and IFN- $\gamma$ on tumor cells treated with conventional PDT [36-38], but their increase or decrease seems to highly depend on the cell line and PDT protocol used. Of note, we observed a small but significant decrease of IL-6 release only after mild NBPDT, which, incidentally, has also been reported after a sublethal PDT dose [36]. This was an unexpected observation and further study will help elucidate this aspect. On the whole, our results point towards an immunostimulatory cytokine profile of the NB-PDT treated tumor cells.

The key element of ICD is the stimulation of immune cells after the release/exposure of immunogenic molecules from dying cells. In this context, DCs are of 
particular interest due to their ability to bridge innate and adaptive immune response. We have confirmed that indeed moDCs show maturation features when in the presence of supernatant of tumor cells treated with the highly cytotoxic NB-PDT, including upregulation of the maturation markers CD86 and MHC II (Figure 5a,b) and increased secretion of IL12-p40, IL-1 $\beta$, and IL-10 (Figure 5c,d). It is important to mention that there exist considerable intrinsic differences between donors, which impacts the statistical significance of this set of experiments. Interestingly, we have observed a similar upregulation pattern of maturation markers on moDCs (although statistically nonsignificant) when using conditioned medium of treated scc-U8 cells in a small scale experiment (Figure S4), suggesting a more moderate damage to the tumor cells may lead as well to phenotypic maturation of moDCs. Nevertheless, conclusions should be carefully drawn from such a small scale experiment. Others have reported maturation of DCs induced by conventional PDT, including upregulation of CD40, CD80, CD83, CD86, and MHC II, accompanied by increased secretion of IL-1 $\beta$, IL-12, nitric oxide, and IFN- $\gamma[26,30,31,34,39]$. Although secretion of IL-10 (an immunosuppressive cytokine) has been reported as reduced or absent in some studies [26,34], others like ours show low levels of this cytokine are still secreted by PDT-stimulated DCs [31]. In our case, this might be explained by the fact that the NB-PDT treated tumor supernatants present TLR4 agonist activity (Figure S5), in agreement with the increase of HSP70 and HMGB1 that are TLR4 agonists themselves and can trigger IL-10 release [27].

A crucial event for antitumor immunity is the subsequent activation of naïve $T$ cells by mature DCs. We describe that moDCs stimulated by tumor supernatant of the highly cytotoxic NB-PDT condition are able to provide the adequate stimulatory signals for the proliferation of CD4+ T cells (Figure 6a) and differentiation towards Th1 CD4+ effector $\mathrm{T}$ cells, as indicated by the substantial release of IFN $\gamma$ (Figure 6b). Th1 CD4+ effector T cells are known to have a clear antitumor role and, via IFN $\gamma$ release, can sustain a potent cellular immune response involving CD8+ cytotoxic T cells [40,41]. Although the activation of these cytotoxic $\mathrm{T}$ cells was not investigated in our study, their importance for tumor control is evident and we will further expand on this aspect in future in vivo studies. In line with our findings, induction of $\mathrm{T}$ cell proliferation and activation in vitro has also been described after conventional PDT [26,31,42].

One important aspect to highlight is that although NB-PDT induces a large extent of necrosis, the immunogenicity here observed is not purely a passive effect of accidental necrosis and subsequent cell content release. It has been shown that cell lysates obtained by the freeze-thaw method, mimicking accidental necrosis, often fail to induce a marked upregulation of surface maturation markers on DCs and to activate T cells, while inducing very high levels of secreted IL-10 by DCs [26,31,34]. This supports the fact that NB-PDT leads to tumor cell death with a distinct immunogenic profile than mere accidental necrosis. Although the exact mechanism of how a PS localized on the cell membrane leads to ICD is still not fully elucidated, some clues have been given for antibody-targeted PDT, which can likely also be valid for NB-PDT. It has been shown that antibodytargeted PDT induces tiny perforations on the plasma membrane, followed by ionic imbalance and increase in membrane permeability, leading to cell rupture, necrosis and ICD [43]. In the same line, it has also been reported that the anticancer peptide RT53 induces a non-regulated form of necrosis which is immunogenic, including disruption of plasma membrane, release of intracellular content, and chaperone exposure via a distinct mechanism which does not follow the "canonical" pathways elicited by other ICD inducers [44]. In these cases, such as with NB-PDT, ICD is suggested to begin with cell membrane damage, possibly leading to ionic imbalance and the surface exposure of 
chaperones, which has been suggested to be controlled by endoplasmic reticulum $\mathrm{Ca} 2+$ levels [45], though additional studies should explore this further.

Altogether, our in vitro study shows that the presence of ICD markers and activation of immune cells induced by NB-PDT is more robust and pronounced when using a tumor cell line with high expression levels of the target. We expect that NB-PDT on tumor cells with moderate target expression will lead to more pronounced effects in vivo than in vitro, where tumor vasculature damage also plays a role. It has already been demonstrated that NB-PDT induces extensive tumor damage on a model with moderate expression levels [7] and, in addition, antibody-targeted PDT has been reported to trigger antitumor immunity against tumor cells with moderate levels of target [46]. Another aspect to underline from our study is the moderate immunogenicity induced by the mild NB-PDT condition. Others have used the treated tumor cells themselves as the initial stimulant $[26,30,31,34,39]$, while we are the first to rely on the immunomodulatory effects of the conditioned medium of the treated cells, without the use of immunoadjuvants [47]. In our setup, the importance of released immunogenic factors plays a more essential role, which is known to abundantly happen in cells dying by necrosis (highly cytotoxic NB-PDT) in comparison to (immunogenic) apoptotic cells. The mild NB-PDT might rely on a combination of released and exposed molecules on the membrane of dying cells and a coincubation setup may yield more promising results. Others have focused their efforts on using PDT doses inducing $\sim 90 \%$ of cell death for the stimulation of DCs, often with the goal of developing antitumor vaccines, thus comparison is difficult in this context. Interestingly, suboptimal PDT protocols in vivo are potent immunostimulants despite the initial low tumor damage [48], thus it seems reasonable to believe that NB-PDT doses resulting in lower cytotoxicity can also lead to immunostimulation in the in vivo setting.

In conclusion, we have shown that NB-PDT holds the potential to stimulate the immune system. This can make an initial local treatment, which causes extensive damage to the primary tumor, also a systemic treatment capable of fighting metastases and preventing recurrences. The initial steps reported here pave the way for a series of events which are deemed of paramount importance if NB-PDT is to be further developed towards the clinic. Our next efforts will be directed towards investigating the immune effects of NB-PDT in preclinical models, to determine the extent of the systemic effects induced and to assess the development of an antitumor immunity.

\section{Conclusions}

Our results show, for the first time, that NB-PDT induces rapid necrosis leading to the exposure and release of DAMPs and cytokines from dying tumor cells. Consequently, maturation of DCs and T cell activation is induced. These findings suggest that NB-PDT, like conventional PDT, can trigger ICD and potentially stimulate the immune system, providing systemic effects to a selective and local therapy.

\section{Acknowledgments}

Authors thank Frederique van den Haak and Julien Bensoussan for their contribution to the initial phase of this study. They also thank Frank Redegeld, Verónica Ayechu Muruzabal and other members of the Pharmacology division of Utrecht University for their support and sharing of facilities. 


\section{References}

1. van Straten, D.; Mashayekhi, V.; de Bruijn, H.S.; Oliveira, S.; Robinson, D.J. Oncologic photodynamic therapy: Basic principles, current clinical status and future directions. Cancers (Basel). 2017, 9, 1-54.

2. Dolmans, D.E.J.G.J.; Fukumura, D.; Jain, R.K. Photodynamic therapy for cancer. Nat Rev Cancer 2003, 3, 380-387.

3. Castano, A.P.; Mroz, P.; Hamblin, M.R. Photodynamic therapy and anti-tumour immunity. Nat Rev Cancer 2006, 6, 535-545.

4. Beltrán Hernández, I.; Yu, Y.; Ossendorp, F.; Korbelik, M.; Oliveira, S. Preclinical and Clinical Evidence of Immune Responses Triggered in Oncologic Photodynamic Therapy: Clinical Recommendations. J. Clin. Med. 2020, 9, 333.

5. Agostinis, P.; Berg, K.; Cengel, K..; Foster, T..; Girotti, A..; Gollnick, S..; Hahn, S..; Hamblin, M..; Juzeniene, A.; Kessel, D.; et al. Photodynamic Therapy of cancer: an update. CA Cancer J Clin. 2011, 61, 250-281.

6. Cognetti, D.; Curry, J.M.; Gillenwater, A.M.; William, W.N.; Kochuparambil, S.T.; McDonald, D.; Fidler, M.; Stenson, K.M.; Vasan, N.R.; Razaq, M.A.; et al. A Phase 2a, Multicenter, OpenLabel Study of RM-1929 Photoimmunotherapy in Patients With Recurrent Head And Neck Cancer. Int. J. Radiat. Oncol. 2018, 100, 1368.

7. Van Driel, P.B.A.A.; Boonstra, M.C.; Slooter, M.D.; Heukers, R.; Stammes, M.A.; Snoeks, T.J.A.; De Bruijn, H.S.; Van Diest, P.J.; Vahrmeijer, A.L.; Van Bergen En Henegouwen, P.M.P.; et al. EGFR targeted nanobody-photosensitizer conjugates for photodynamic therapy in a pre-clinical model of head and neck cancer. J. Control. Release 2016, 229, 93-105.

8. Heukers, R.; van Bergen en Henegouwen, P.M.P.; Oliveira, S. Nanobody-photosensitizer conjugates for targeted photodynamic therapy. Nanomedicine Nanotechnology, Biol. Med. 2014, 10, 1441-1451.

9. Oliveira, S.; Heukers, R.; Sornkom, J.; Kok, R.J.; van Bergen En Henegouwen, P.M.P. Targeting tumors with nanobodies for cancer imaging and therapy. J. Control. Release 2013, 172, 607-17.

10. Oliveira, S.; Van Dongen, G. a M.S.; Stigter-Van Walsum, M.; Roovers, R.C.; Stam, J.C.; Mali, W.; Van Diest, P.J.; Van Bergen En Henegouwen, P.M.P. Rapid visualization of human tumor xenografts through optical imaging with a near-infrared fluorescent anti-epidermal growth factor receptor nanobody. Mol. Imaging 2012, 11, 33-46.

11. Beltrán Hernández, I.; Rompen, R.; Rossin, R.; Xenaki, K.T.; Katrukha, E.A.; Nicolay, K.; van Bergen en Henegouwen, P.; Grüll, H.; Oliveira, S. Imaging of Tumor Spheroids, Dual-Isotope SPECT, and Autoradiographic Analysis to Assess the Tumor Uptake and Distribution of Different Nanobodies. Mol. Imaging Biol. 2019, 21, 1079-1088.

12. Deken, M.M.; Kijanka, M.M.; Beltrán Hernández, I.; Slooter, M.D.; de Bruijn, H.S.; van Diest, P.J.; van Bergen en Henegouwen, P.M.; Lowik, C.W.; Robinson, D.J.; Vahrmeijer, A.L.; et al. Nanobody-targeted photodynamic therapy induces significant tumor regression of trastuzumabresistant HER2-positive breast cancer, after a single treatment session. J. Control. Release 2020, 323, 269-281.

13. Heukers, R.; Mashayekhi, V.; Ramirez-Escudero, M.; de Haard, H.; Verrips, T.C.; van Bergen en Henegouwen, P.M.P.; Oliveira, S. VHH-Photosensitizer Conjugates for Targeted Photodynamic Therapy of Met-Overexpressing Tumor Cells. Antibodies (Basel) 2019, 8, 26.

14. De Groof, T.W.M.; Mashayekhi, V.; Shu Fan, T.; Bergkamp, N.D.; Sastre Torano, J.; Van Senten, J.R.; Heukers, R.; Smit, M.J.; Oliveira, S. Nanobody-Targeted Photodynamic Therapy Selectively Kills Viral GPCR-Expressing Glioblastoma Cells. Mol. Pharm. 2019, 16, 3145-3156.

15. de Bruijn, H.S.; Mashayekhi, V.; Schreurs, T.J.L.; van Driel, P.B.A.A.; Strijkers, G.J.; van Diest, P.J.; Lowik, C.W.G.M.; Seynhaeve, A.L.B.; Hagen, T.L.M. ten; Prompers, J.J.; et al. Acute cellular and vascular responses to photodynamic therapy using EGFR-targeted nanobody-photosensitizer conjugates studied with intravital optical imaging and magnetic resonance imaging. Theranostics 2020, 10, 2436-2452. 
16. Garg, A.D.; Agostinis, P. ER stress, autophagy and immunogenic cell death in photodynamic therapy-induced anti-cancer immune responses. Photochem. Photobiol. Sci. 2014, 13, 474-87.

17. Garg, A.D.; Galluzzi, L.; Apetoh, L.; Baert, T.; Zitvogel, L.; Agostinis, P. Molecular and translational Classifications of DaMPs in immunogenic Cell Death. Front. Immunol. 2015, 6.

18. Maeding, N.; Verwanger, T.; Krammer, B. Boosting Tumor-Specific Immunity Using PDT. 2016.

19. Peng, W.; de Bruijn, H.S.; Farrell, E.; Sioud, M.; Mashayekhi, V.; Oliveira, S.; van Dam, G.M.; Roodenburg, J.L.N.; Witjes, M.J.H.; Robinson, D.J. Epidermal growth factor receptor (EGFR) density may not be the only determinant for the efficacy of EGFR-targeted photoimmunotherapy in human head and neck cancer cell lines. Lasers Surg. Med. 2018, 50, 513-522.

20. Driehuis, E.; Spelier, S.; Beltrán Hernández, I.; De Bree, R.; Willems, S.M.; Clevers, H.; Oliveira, S. Patient-Derived Head and Neck Cancer Organoids Recapitulate EGFR Expression Levels of Respective Tissues and Are Responsive to EGFR-Targeted Photodynamic Therapy. J. Clin. Med. 2019, 8, 1880.

21. Anzengruber, F.; Avci, P.; De Freitas, L.F.; Hamblin, M.R. T-cell mediated anti-tumor immunity after photodynamic therapy: Why does it not always work and how can we improve it? Photochem. Photobiol. Sci. 2015.

22. Heukers, R.; Vermeulen, J.F.; Fereidouni, F.; Bader, A.N.; Voortman, J.; Roovers, R.C.; Gerritsen, H.C.; Van Bergen En Henegouwen, P.M.P. Endocytosis of EGFR requires its kinase activity and N-terminal transmembrane dimerization motif. J. Cell Sci. 2013, 126, 4900-4912.

23. Nicotera, P.; Leist, M.; Ferrando-May, E. Intracellular ATP, a switch in the decision between apoptosis and necrosis. Toxicol. Lett. 1998, 102-103, 139-142.

24. Thompson, S.A.; Aggarwal, A.; Singh, S.; Adam, A.P.; Tome, J.P.C.; Drain, C.M. Compromising the plasma membrane as a secondary target in photodynamic therapy-induced necrosis. Bioorganic Med. Chem. 2018, 26, 5224-5228.

25. Ogawa, M.; Tomita, Y.; Nakamura, Y.; Lee, M.; Lee, S.; Tomita, S.; Nagaya, T.; Sato, K.; Yamauchi, T.; Trepel, B.; et al. Immunogenic cancer cell death selectively induced by near infrared photoimmunotherapy initiates host tumor immunity. Oncotarget 2017, 8, 10425-10436.

26. Ji, J.; Fan, Z.; Zhou, F.; Wang, X.; Shi, L.; Zhang, H.; Wang, P.; Yang, D.; Zhang, L.; Chen, W.R.; et al. Improvement of DC vaccine with ALA-PDT induced immunogenic apoptotic cells for skin squamous cell carcinoma. Oncotarget 2015, 6, 17135-17146.

27. Garg, A.D.; Nowis, D.; Golab, J.; Vandenabeele, P.; Krysko, D. V; Agostinis, P. Immunogenic cell death, DAMPs and anticancer therapeutics : An emerging amalgamation. BBA-Rev. Cancer 2010, $1805,53-71$.

28. Panzarini, E.; Inguscio, V.; Fimia, G.M.; Dini, L. Rose Bengal Acetate PhotoDynamic Therapy ( RBAc-PDT ) Induces Exposure and Release of Damage-Associated Molecular Patterns ( DAMPs ) in Human HeLa Cells. 2014, 9, 20-22.

29. Korbelik, M.; Sun, J.; Cecic, I. Photodynamic Therapy - Induced Cell Surface Expression and Release of Heat Shock Proteins : Relevance for Tumor Response. Cancer Res. 2005, 65, 10181026.

30. Wang, X.; Ji, J.; Zhang, H.; Fan, Z.; Zhang, L.; Shi, L.; Zhou, F.; Chen, W.R.; Wang, H.; Wang, $\mathrm{X}$. Stimulation of dendritic cells by DAMPs in ALA-PDT treated SCC tumor cells. Oncotarget 2015, 6, 44688-44702.

31. Zheng, Y.; Yin, G.; Le, V.; Zhang, A.; Chen, S.; Liang, X.; Liu, J. Photodynamic-therapy Activates Immune Response by disrupting Immunity Homeostasis of Tumor Cells, which Generates Vaccine for Cancer Therapy. Int. J. Biol. Sci. 2016, 12, 120-132.

32. Tesniere, A.; Panaretakis, T.; Kepp, O.; Apetoh, L.; Ghiringhelli, F.; Zitvogel, L.; Kroemer, G. Molecular characteristics of immunogenic cancer cell death. Cell Death Differ. 2008, 15, 3-12.

33. Tanaka, M.; Kataoka, H.; Yano, S.; Sawada, T. Immunogenic cell death due to a new photodynamic therapy ( PDT ) with glycoconjugated chlorin ( G-chlorin ). Oncotarget 2016, 7, 47242-47251.

34. Garg, A.D.; Krysko, D. V; Verfaillie, T.; Kaczmarek, A.; Ferreira, G.B.; Marysael, T.; Rubio, N.; Firczuk, M.; Mathieu, C.; Roebroek, A.J.M.; et al. A novel pathway combining calreticulin exposure and ATP secretion in immunogenic cancer cell death. EMBO J. 2012, 31, 1062-1079. 
35. Waugh, D.J.J.; Wilson, C. The Interleukin-8 Pathway in Cancer. Clin. Cancer Res. 2008, 14, 6735 6742 .

36. Kawczyk-Krupka, A.; Czuba, Z.; Latos, W.; Wasilewska, K.; Verwanger, T.; Krammer, B.; Siero, A. Influence of ALA-mediated photodynamic therapy on secretion of interleukins 6,8 and 10 by colon cancer cells in vitro. Photodiagnosis Photodyn. Ther. 2018, 22, 137-139.

37. Usuda, J.; Okunaka, T.; Furukawa, K.; Tsuchida, T.; Kuroiwa, Y.; Ohe, Y.; Saijo, N.; Nishio, K.; Konaka, C.; Kato, H. Increased cytotoxic effects of photodynamic therapy in IL-6 gene transfected cells via enhanced apoptosis. Int. J. Cancer 2001, 93, 475-480.

38. Koon, H.; Lo, K.; Leung, K.; Lung, M.L.; Chang, C.C.; Wong, R.N.; Leung, W.; Mak, N. Photodynamic therapy-mediated modulation of inflammatory cytokine production by Epstein Barr virus-infected nasopharyngeal carcinoma cells. Cell. Mol. Immunol. 2010, 7, 323-326.

39. Gollnick, S.O.; Vaughan, L.; Henderson, B.W. Generation of Effective Antitumor Vaccines Using Photodynamic Therapy. Cancer Res. 2002, 62, 1604-1608.

40. Schoenborn, J.R.; Wilson, C.B. Regulation of Interferon- g During Innate and Adaptive Immune Responses. Adv. Immunol. 2007, 96, 41-101.

41. Galaine, J.; Borg, C.; Godet, Y.; Adotévi, O. Interest of Tumor-Specific CD4 T Helper 1 Cells for Therapeutic Anticancer Vaccine. Vaccines 2015, 3, 490-502.

42. Garg, A.D.; Dudek, A.M.; Ferreira, G.B.; Verfaillie, T.; Vandenabeele, P.; Krysko, D. V; Mathieu, C.; Agostinis, P.; Garg, A.D.; Dudek, A.M.; et al. ROS-induced autophagy in cancer cells assists in evasion from determinants of immunogenic cell death. Autophagy 2013, 9, 1292-1307.

43. Nakajima, K.; Takakura, H.; Shimizu, Y.; Ogawa, M. Changes in plasma membrane damage inducing cell death after treatment with near-infrared photoimmunotherapy. Cancer Sci. 2018, 109, 2889-2896

44. Pasquereau-kotula, E.; Habault, J.; Kroemer, G.; Poyet, J.-L. The anticancer peptide RT53 induces immunogenic cell death. PLoS One 2018, 13, e0201220.

45. Tufi, R.; Panaretakis, T.; Bianchi, K.; Criollo, A.; Fazi, B.; Sano, F. Di; Tesniere, A.; Kepp, O.; Paterlini-Brechot, P.; Zitvogel, L.; et al. Reduction of endoplasmic reticulum Ca2+ levels favors plasma membrane surface exposure of calreticulin. Cell Death Differ. 2008, 15, 274-282.

46. Nagaya, T.; Friedman, J.; Maruoka, Y.; Ogata, F.; Okuyama, S.; Clavijo, P.E.; Choyke, P.L.; Allen, C.; Kobayashi, H. Host immunity following near-infrared photoimmunotherapy is enhanced with PD-1 checkpoint blockade to eradicate established antigenic tumors. Cancer Immunol. Res. 2019, 7, 401-413.

47. Xia, Y.; Gupta, G.K.; Castano, A.P.; Mroz, P.; Avci, P.; Hamblin, M.R. CpG oligodeoxynucleotide as immune adjuvant enhances photodynamic therapy response in murine metastatic breast cancer. J. Biophotonics 2014.

48. Henderson, B.W.; Gollnick, S.O.; Snyder, J.W.; Busch, T.M.; Kousis, P.C.; Cheney, R.T.; Morgan, J. Choice of Oxygen-Conserving Treatment Regimen Determines the Inflammatory Response and Outcome of Photodynamic Therapy of Tumors. Cancer Res. 2004, 64, 2120-2126.

49. Haigler, H.; Asht, J.F.; Singert, S.J.; Cohen, S. Visualization by fluorescence of the binding and internalization of epidermal growth factor in human carcinoma cells A-431. Proc. Natl. Acad. Sci. USA 1978, 75, 3317-3321 


\section{Supplementary Material}

(a)

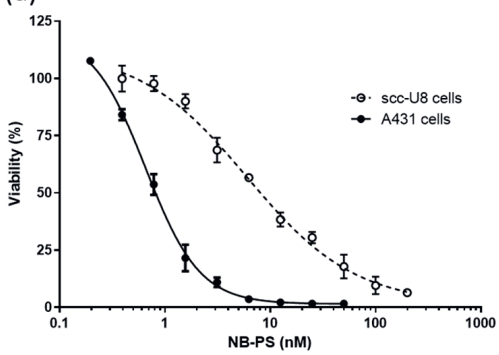

(b)

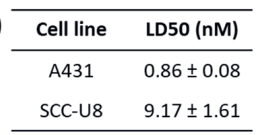

(c)

\begin{tabular}{ccc}
\hline Cell line & LD50 (nM) & LD100 (nM) \\
\hline A431 & 1 & 25 \\
SCC-U8 & 10 & 100 \\
\hline
\end{tabular}

Figure S1 Cytotoxicity induced by NB-PDT on different cell lines. A431 and scc-U8 cells were treated with NBPDT with a concentration range of 7D12-PS conjugate. Cells were placed back in the incubator and cell viability was assessed 24 hours after the treatment using AlamarBlue reagent. (a) Cell viability curves (\% relative to untreated cells) from which LD50 values were calculated. (b) Overview of the LD50 values for each cell line. Differences in the cytotoxicity of the treatment on the different cell lines are explained by the 3-fold higher EGFR levels on A431 cells. (c) Overview of the concentrations to achieve LD50 and LD100 used in this study for each cell line.

(a)

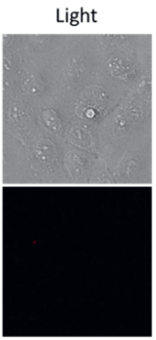

NB-PS

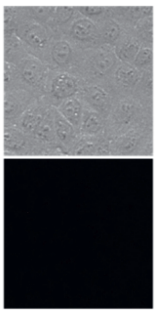

(b)

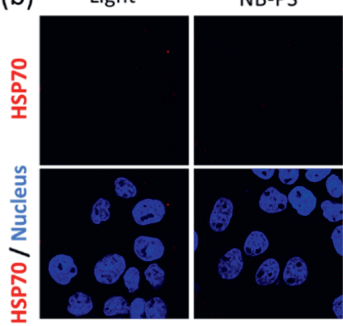

(c)

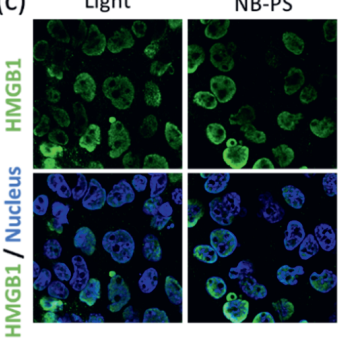

Figure S2 NB-PDT controls on A431 cells for different assays. (a) Tumor cells were subjected to either only light or only 7D12-PS conjugate (LD100) and stained with PI for necrotic cells and caspase 3/7 for apoptotic cells. Microscopy images were taken 18 hours later. Top panels depict the transmitted light image, while merged images of necrotic and apoptotic cells are shown on the bottom panels. (b) and (c) Tumor cells were subjected to the single components of PDT. Four hours later, either (b) extracellular staining of HSP70 (red) or (c) intracellular staining of HMGB1 (green) was performed. Cell nuclei were additionally stained with DAPI (blue). Top panels depict only the DAMP signal, while merged images are shown on the bottom panels. The same controls reported in this figure were also performed with scc-U8 cells, resulting in similar images. 
(a)

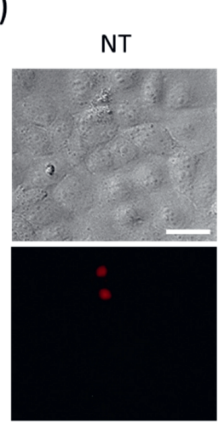

(b)

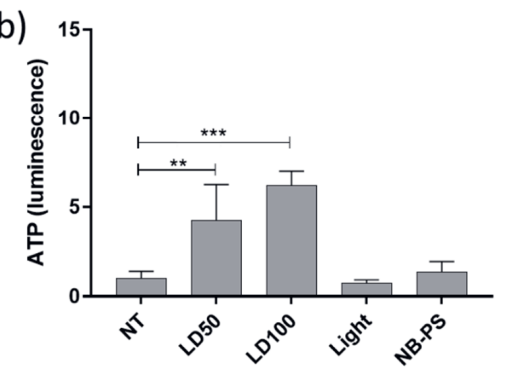

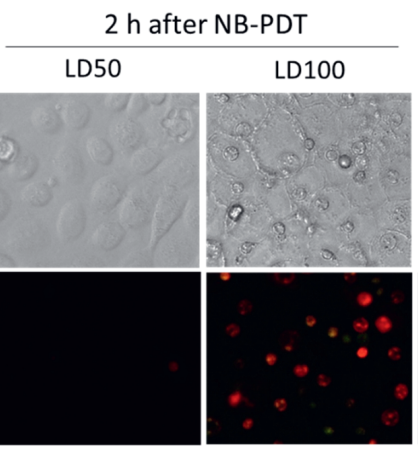

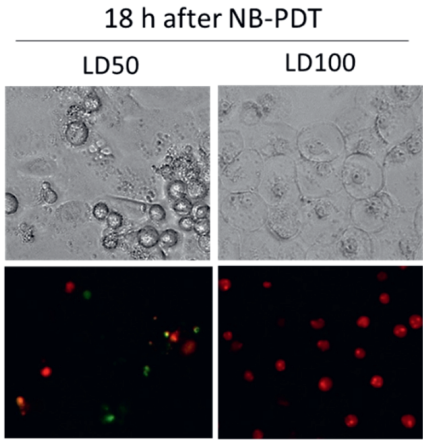

(c)

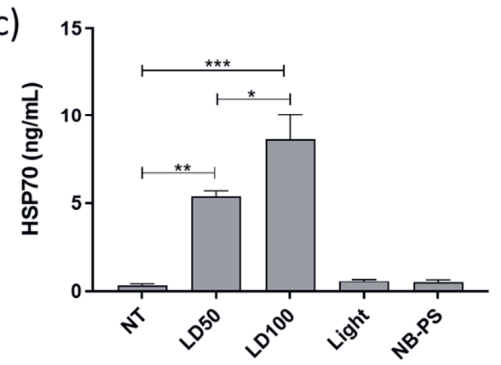

Figure S3 Effect of NB-PDT with the biparatopic conjugate 7D12-9G8-PS on tumor cells. (a) A431 cells were left untreated (NT) or treated with NB-PDT (LD50 or LD100) and stained with PI for necrotic cells (red) and caspase 3/7 for apoptotic cells (green). Microscopy images were taken 2 and 18 hours after the treatment. Top panels depict the transmitted light image, while merged images of necrotic and apoptotic cells are shown on the bottom panels. Cells that are stained with both dyes appear orange and represent apoptotic cells at a later stage. Scale bar, $20 \mu \mathrm{m}$. (b) and (c) A431 cells were left untreated or treated with NB-PDT (or controls consisting of only light or NB-PS conjugate). ATP in the supernatant was detected 4 hours later via a luminescence assay and graphs show luminescence values relative to untreated cells (b). Additionally, HSP70 in the supernatant was detected 24 hours after treatment using ELISA (c). Significance is displayed as $* \mathrm{p} \leq 0.05, * * \mathrm{p} \leq 0.01$ and $* * * \mathrm{p} \leq 0.001$.

(a)

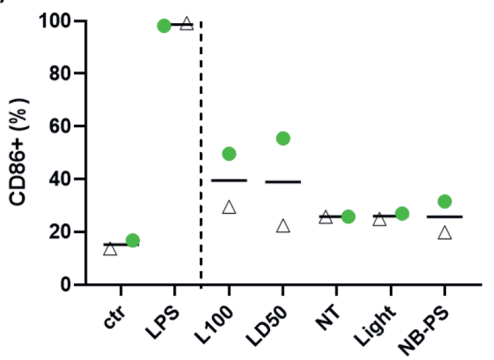

(b)

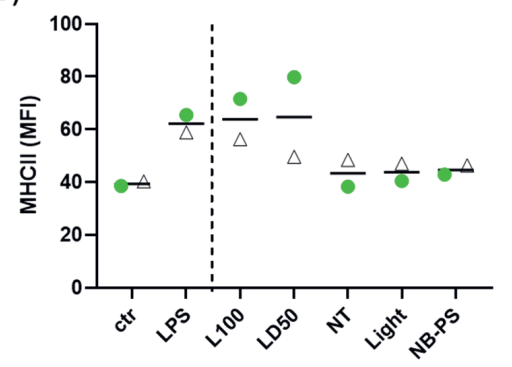

Figure S4 Phenotypic maturation of moDCs incubated with supernatant of NB-PDT treated sccU8 cells. scc-U8 cells were left untreated (NT) or treated with NB-PDT using 7D12-PS (LD50 or LD100) and the respective controls (only light or NB-PS conjugate). The supernatant was 
collected 24 hours later and incubated with immature moDCs for another 24 hours. Surface marker expression on moDCs was measured with flow cytometry. A positive control was obtained by incubating moDCs with $100 \mathrm{ng} / \mathrm{ml}$ LPS for 24 hours (LPS), and a control consisting of unstimulated moDCs (ctr) was included. (a) Percentage of CD86 positive moDCs. (b) Median fluorescence intensity (MFI) corresponding to MHC II expression on moDCs. Each donor is represented by a different symbol $(n=2)$.

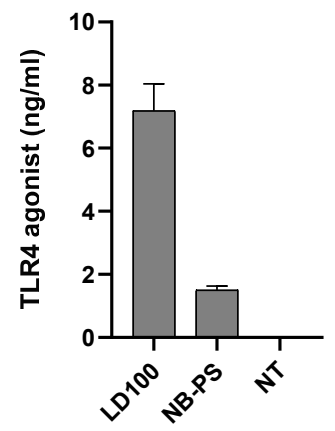

Figure S5 Tumor supernatant after NB-PDT presents TLR4 agonistic activity. A431 cells were left untreated (NT), treated with NB-PDT using 7D12-PS (LD100) or a control with only conjugate (NB-PS). The supernatant was collected 24 hours later and used in a reporter assay with the cell line HEK-Blue-hTLR4 cells (InvivoGen, hkb-htlr4) following the manufacturer's protocol. Tumor supernatant after treatment with highly cytotoxic NB-PDT showed TLR4 agonistic activity equivalent to $7.2 \mathrm{ng} / \mathrm{ml}$ of LPS. 
Nanobody-targeted PDT triggers immune responses 

CHAPTER 8

Summary and perspectives 


\section{Summary}

Cancer is a highly heterogeneous disease and, as such, its treatment presents one of the greatest scientific and medical challenges. Mainstream therapeutic options are often accompanied by side effects and have substantial impact on the quality of life of patients. Efforts have been made in the direction towards novel tumor-selective approaches, with the idea to minimize damage to healthy tissues. In this thesis we focus on an approach developed over the last 7 years: nanobody-targeted photodynamic therapy (NB-PDT). This targeted approach makes use of nanobodies (NBs), which are the smallest naturally occurring antigen binding domains. The NBs guide the selective delivery of a photosensitizer (PS) to the tumor cells, a molecule that upon local light application leads to the formation of reactive oxygen species and consequent cell death. NB-PDT has so far shown promise in vitro and in orthotopic mouse models using different molecular targets. As the next step, this thesis focuses on positioning NB-PDT one step closer to the clinic.

In Chapter 1, a general introduction on the current cancer therapies in the clinic is provided, emphasizing the limitations of these and need for new approaches. Photodynamic therapy (PDT) is presented as a tumor-selective and minimally invasive strategy, but still underutilized in the clinic. Attempts to improve PDT by introducing a targeting component in the approach are discussed, namely antibody-targeted PDT (socalled photoimmunotherapy or PIT) and NB-PDT. PIT is currently under evaluation in a phase III clinical trial for the treatment of head and neck squamous cell carcinoma (HNSCC), using a PS conjugated to cetuximab, a commercial monoclonal antibody $(\mathrm{mAb})$ targeting the epidermal growth factor receptor (EGFR). As a step forward in the improvement of PDT, using a small targeting molecule, such as a NB, brings advantages. In particular, a more rapid and homogeneous distribution throughout tumors, and rapid renal clearance enabling faster start of the illumination after PS administration and reducing the chances of photosensitivity after treatment. HNSCC and the presence of EGFR in these cancers are also discussed in detail in this chapter, as a type of cancer currently treated by PDT and PIT, and as the focus for the further development of NBPDT. Bearing in mind the clinical translation of NB-PDT, the importance of clinically relevant models to be able to faithfully study the potential of anticancer therapies is also highlighted.

One limiting aspect of PIT is the heterogeneous distribution of the mAb-PS conjugate throughout the tumor, due to the large molecular size of the mAb $(\sim 150 \mathrm{kDa})$. NBs are small $(\sim 15 \mathrm{kDa})$, but easily engineered to create multivalent constructs, e.g. to increase avidity or half-life. Therefore, in Chapter 2, we explored the effect of molecular size on the tumor penetration of NBs and mAbs. First, the tumor distribution of the irrelevant NB R2 (16 kDa), the EGFR-targeting NBs 7D12 (16 kDa) and 7D12-R2 (32 $\mathrm{kDa})$, and the EGFR-targeting mAb cetuximab $(150 \mathrm{kDa})$ was investigated, using tumor spheroids $(150-200 \mu \mathrm{m})$ grown from EGFR-overexpressing A431 tumor cells. While homogeneous distribution of 7D12 and 7D12-R2 throughout the spheroids was achieved in 2 and $4 \mathrm{~h}$, respectively, this was only observed for the mAb after $24 \mathrm{~h}$. To study tumor uptake and distribution of the different NBs in mice bearing A431 tumors, dual-isotope single-photon emission computed tomography (SPECT) was employed for the first time for NBs, allowing longitudinal and simultaneous detection of two NBs in a single animal. The tumor uptake of 7D12 was 2.5-fold higher than 7D12-R2 when the NB pairs were co-injected. Ex vivo autoradiographic analysis of tumors sections revealed that indeed 7D12 was more evenly distributed throughout the tumor, while the larger NB was localized mainly at the tumor rim. This study revealed that increasing the size of the NB 
format, even just by $15 \mathrm{kDa}$, hinders their tumor penetration. In particular, it becomes clear that monomeric NBs are a superior choice for molecular imaging and, most likely, also for targeted PDT.

In Chapter 3, we indeed moved towards the field of targeted PDT and the use of clinically relevant 3D models with translational potential: organoids grown from both HNSCC patient material and corresponding adjacent normal tissue. First, the membrane EGFR levels on the organoids were found to recapitulate the EGFR expression in the primary tissue, a very important feature since common HNSCC cell lines tend to misrepresent expression levels. The potency of EGFR-targeted PDT was then investigated using NB-PS conjugates which have previously shown good in vitro and in vivo results (i.e. 7D12-PS and the biparatopic 7D12-9G8-PS), and cetuximab-PS, identical to the mAb-PS conjugate tested in the clinic. Although Chapter 2 discourages the use of dimeric NBs in this context, 7D12-9G8-PS was taken along because of its higher potency in vitro and comparable in vivo results to 7D12-PS. The organoid technology allowed to explore the specificity of targeted PDT by comparing matched tumor and normal organoids. EGFR levels were lower in the normal counterparts and these were not affected by the treatment. In fact, the response to EGFR-targeted PDT correlated with the EGFR levels on the organoids of the different donors. In general, NBPDT was more effective in inducing cell death than PIT, highlighting the superiority of NBs for targeted PDT. Due to the small size of the organoids $(<70 \mu \mathrm{m})$, the tumor distribution advantage of a monomeric over a larger NB was not apparent and the biparatopic NB-PS conjugate showed higher potency, explained by higher binding affinity, avidity, PS molecules per NB and internalization rate. Importantly, this study presented HNSCC organoids as a more realistic representation of tumors than commonly used cell lines.

The encouraging results of NB-PDT efficacy in HNSCC organoids prompted the further development of NB-PDT towards the human clinic. In this direction, Chapter 4 explores the introduction of companion animals with spontaneous tumors in the current drug development programs to aid therapy refinement and increase translational success of anticancer targeted therapies. At the same time, these veterinary patients gain access to novel, otherwise inaccessible, therapies. This chapter provides an overview of targetable membrane proteins expressed in the most well-characterized canine and feline models of solid cancer, which greatly resemble the counterpart human malignancies: feline oral squamous cell carcinoma (OSCC), canine bladder cancer, canine and feline mammary carcinoma, canine osteosarcoma and canine melanoma. Moreover, considerations and challenges to implement the approach of comparative oncology in the development of anticancer targeted therapies are also discussed. Amongst other targets with great translational potential, EGFR was identified as a candidate to develop targeted therapies against feline OSCC, which is considered a naturally occurring model of human HNSCC and for which there is no curative option so far. EGFR was selected due to its expression in this feline cancer type and the currently successful anti-EGFR strategies to treat HNSCC.

Accordingly, efforts were put in the development of a NB-PS conjugate for the treatment of cats with OSCC. The in vitro work preceding this step in the veterinary clinic is presented in Chapter 5. Starting from NBs previously selected against human EGFR, a NB was identified with the ability to bind both human and feline EGFR with comparable affinity in the single digit nanomolar range. The EGFR-specific binding was confirmed by using a panel of feline and human cell lines as well as feline OSCC tissue, and comparing the detection of EGFR with a commercially available, species cross-reactive, anti-EGFR mAb. The membrane EGFR levels on three feline OSCC cell lines were 
similar to human HeLa cells, which is comparable to the HNSCC organoids used in Chapter 3. In order to induce potent cytotoxicity to cells with such clinically relevant EGFR expression levels, a highly loaded NB-PS conjugate was developed (i.e. NiBh), consisting of a monomeric NB as the ideal format described in Chapter 2. This conjugate, despite the high PS payload, retained high affinity and remained specific for EGFR, as evidenced by competitive binding with EGF. NiBh was successfully employed to induce potent cell death to feline OSCC cell lines (LD50 in low nanomolar range). This cytotoxicity was found to be mediated by the binding of NiBh to EGFR and by the production of singlet oxygen. To resemble the EGFR expression pattern found in feline tumor tissue, which is confined to neoplastic nests and absent in surrounding stroma, feline OSCC cells were co-cultured with low EGFR-expressing MCF7 cells and subjected to PDT using NiBh. This revealed the high specificity of the PDT approach, since feline cancer cells were killed whereas adjacent low EGFR-expressing cells were unharmed. $\mathrm{NiBh}$ is thus presented as a highly loaded, EGFR-targeting, species cross-reactive NB-PS conjugate, suitable for NB-PDT in a selective manner. The encouraging results of this study support the idea to further develop NiBh for the treatment of cats with OSCC.

One very interesting and appealing feature of PDT is its ability to trigger antitumor immune responses. These are not drivers of the initial tumor destruction, but essential for creating a systemic response to fight metastasis and for developing an immune memory to prevent recurrences. Although promising, this antitumor mechanism of PDT is not fully elucidated yet and not always successfully triggered, partly dependent on the original tumor immunogenicity and its immunosuppressive environment. Therefore, further understanding of these immunological responses would make it possible to exploit these responses for stronger and longer effects in the clinic. Chapter 6 reviews the preclinical and clinical evidence regarding this aspect of PDT. In general, an initial local inflammation is always triggered, which may further develop into an adaptive immune response when immunogenic cell death (ICD) has been induced. ICD involves the redistribution and release from dying tumor cells of damage-associated molecular patterns (DAMPs), which are recognized by infiltrating immune cells which subsequently can stimulate and restore antitumor responses. In this context, the combination of PDT with immunomodulatory agents can maximize the therapeutic effect of PDT. For instance, the combination of PDT with vaccine adjuvants or with immune checkpoint inhibitors has been shown to synergize the PDT effect in the preclinical setting. Overall, at present, the evidence of PDT-induced immunological responses in the clinic is unfortunately very scarce. Accordingly, recommendations are given to guide future clinical research and ensure that the immunological potential of PDT is explored in its full, focusing on noninvasive monitoring of the most determinant immune events observed in animals.

It is evident that triggering immune responses would greatly add to the PDT treatment efficacy. For this reason, Chapter 7 focuses on the assessment of the possible immune stimulation induced by NB-PDT in vitro. The NB-PS conjugate 7D12-PS was employed in this study to induce the killing of high (A431) and moderate (scc-U8) EGFRexpressing cells, to resemble to some extent the in vivo tumor heterogeneity of target expression. Conventional PDT is known to predominantly induce apoptosis, due to intracellular localization of the PS and organelle damage. Nevertheless, NB-PDT was shown to mainly induce necrosis on both cell lines. This is explained by the slow internalization of 7D12-PS once bound to EGFR, being mostly present on the cell membrane when light is applied and thereby inducing damage to this cellular structure. Several indicators of ICD were detected after NB-PDT on both cell lines: changes in the cellular localization of HSP70 and HMGB1, and release of HSP70, ATP and pro- 
inflammatory cytokines IL- 6 and IL-1 $\beta$, being the effects always more pronounced on the high EGFR-expressing cells. The conditioned medium of the treated high expressing tumor cells was able to induce maturation of human monocyte-derived dendritic cells, as indicated by the surface upregulation of CD86 and MHC II and the increased release of IL-12 and IL-1 $\beta$. These mature dendritic cells were able to activate allogeneic CD4+ T cells upon cross-linking, inducing T cell proliferation and IFN $\gamma$ release. Altogether, these are the initial steps suggesting immune stimulation triggered by NB-PDT, potentially giving a systemic reach to an initial local treatment. Further characterization of this immunological aspect in in vivo models is warranted.

Taken together, this thesis highlights the value of NBs over mAbs to achieve fast and homogenous distribution throughout tumors, presents the potency of NB-PDT in a clinically relevant model consisting of HNSCC organoids, and describes the in vitro steps towards the application of NB-PDT to treat cats with OSCC. Furthermore, the first signs of immune stimulation triggered by NB-PDT are presented, suggesting the induction of an antitumor mechanism with great clinical implications.

\section{Discussion and perspectives}

\section{General considerations on PDT application}

The main cause of treatment failure for two traditional anticancer therapies (chemo- and radiotherapy) is resistance. Radioresistant tumors are usually large and/or hypoxic tumors, commonly with upregulated DNA repair pathways. On the other hand, altered drug efflux or uptake are associated with chemoresistance [1]. Likewise, the long-term effect of targeted therapies and immune checkpoint inhibitors is hindered by the development of resistance, generally in the form of specific gene mutations driving amplification of the target or related genes to sustain oncogenicity [2]. With regard to PDT, the development of resistance after multiple PDT cycles $(\sim 10)$ in vitro has been reported as well, but the presence of this PDT-resistance phenotype in recurring cancers in the clinic remains to be confirmed, since clinical PDT is usually not preformed more than 2 or 3 times [1,3]. Of note, especially the structure of the PS and its subcellular location are deemed critical in the development of resistance to PDT. As such, over half of the cell lines which have developed resistance to PDT with a particular PS, remain sensitive to a different PS [1]. Only one third of the chemo- and radioresistant cell lines are cross-resistant to PDT, supporting the development of combination strategies [1]. This explains why PDT, in some cases, becomes a curative option for patients who have not responded to chemotherapy and radiation therapy [4].

The strength of PDT relies on its tumor-selectivity in a controllable manner, by locally applying light to activate the PS in the tumor tissue. Moreover, targeted PDT adds another level of specificity to the approach, further improving the safety profile over other treatments. The minimally invasive character of PDT and its remarkable safety profile allow for its repeated application upon tumor persistence or recurrence without cumulative toxicity, contrary to radiotherapy regimens [5]. In addition, PDT does not compromise the future treatment options for residual or recurrent disease, and the possibility of re-treatment with surgery or radiation therapy remains. In general, tumor recurrence after PDT is associated with the intrinsic limitations of this therapy, such as poor light penetration or presence of bulky, aggressive or hypoxic tumors, but not to treatment resistance. Accordingly, repeated PDT treatment is indicated, when necessary, and results in positive clinical outcomes. To exemplify this, PDT is considered a 
comparable modality to the three mainstream strategies in the management of early stage HNSCC, but with far less associated morbidity, even after 2-3 treatment rounds [6].

Despite the unique features that differentiate PDT from standard therapies, PDT is still not routinely applied in the clinic due to a number of limitations. Newer PSs tend to utilize near-infrared light which offers the maximum tissue penetration capacity, but this is still limited $(<1 \mathrm{~cm})$ [7]. To surpass this, efforts are put into improving light delivery by combining optic fibers, light diffusers, endoscopes and/or catheters for interstitial and intraoperative PDT of multiple organs, such as pancreas, prostate and brain [8]. Also tumor hypoxia limits the therapeutic effect and, consequently, researches are focusing on the development of oxygen-independent PSs and tumor oxygenation approaches [9]. Furthermore, complex light and oxygen dosimetry still remains a challenge for any PDT approach.

\section{Advantages and limitations of NB-PDT}

The first reports of targeted PDT using mAbs date back to the early 1980's, but PIT as we currently know it was first described by the group of Kobayashi in 2011 [10]. Less than one decade later, PIT has now reached phase III in an ongoing clinical trial for the treatment of recurrent HNSCC in patients who have failed at least two lines of therapy (NCT03769506). In phase II, overall response rate was 50\%, an improvement over mainstream therapies in this highly pre-treated population [11]. As a further improved version of PIT, NB-PDT was first described in 2014 by the group of Oliveira [12]. The potency of NB-PDT and PIT has been compared side by side in an HNSCC organoid model (Chapter 3) and in an orthotopic mouse model of HNSCC [13], resulting in the superiority of NB-PDT in both cases. The small size and rapid renal clearance of the NBPS conjugates are the basis of the main advantage of this therapy over its counterpart utilizing mAbs, allowing for rapid illumination after PS administration, more homogeneous distribution through the tumor tissue and minimal skin photosensitivity post-treatment.

One the other hand, the small size of NBs supposes, at the same time, a limitation in terms of the amount of payload that can be delivered per single NB molecule. While the mAbs employed for PIT can easily carry an average of 3 PS molecules per mAb, monomeric NBs are mostly limited to one PS molecule. Dimeric or larger NB-based constructs enable the incorporation of more PS molecules by means of, for instance, conjugation directed to the primary amines present at the $\mathrm{N}$-terminus and lysine residues. However, as shown in Chapter 2 and supported by literature [14,15], the increase in size of different NB formats (e.g. biparatopic and bispecific NBs) affects their distribution through tumors. Interestingly, this aspect does not seem to negatively impact the potency of NB-PDT in the evaluated in vivo models so far $[13,16]$, although the use of monomeric NB-PS conjugates does result in a more consistent extent of tumor damage. In Chapter 5 of this thesis, we describe a monomeric NB-PS conjugate with high PS load, i.e. average of 2.5 PS molecules per NB, to increase the induced cytotoxicity while retaining high binding affinity. Nevertheless, the application to other NBs of this type of PS conjugation remains to be explored. It is clear that conjugates with high payload lead to larger degree of cytotoxicity in vitro, but the relevance of this in the in vivo setting still needs to be elucidated, where damage to tumor vasculature and stimulation of immune responses also play a role. Moreover, a high PS load can affect the distribution and tumor uptake of the conjugate [17], all aspects that are being explored in ongoing mice studies.

An interesting feature of membrane-targeted PDT is that resistance mechanisms such as altered drug uptake/efflux and activating mutations in signaling pathways, which suppose a hurdle for chemotherapy and targeted therapies, would not hinder the effects 
of NB-PDT. In particular, EGFR-targeted PDT seems to rely more on the mere presence of the target, as evidenced by the correlation of membrane EGFR levels and PDT sensitivity described in Chapter 3. For EGFR-targeted PDT, EGFR is probably a predictive biomarker of response, while this is not the case for other EGFR inhibitory therapies whose effectivity is subjected to downstream alterations (e.g. cetuximab which blocks receptor signaling) [18]. Hence, patient classification by EGFR expression in tumor tissue can likely guide the successful application of targeted PDT. Of note, one feature that could affect the efficacy of targeted PDT is the intratumoral heterogeneous expression of the utilized membrane target, or the adaptive downregulation of such. At this point is when the three distinct, but interdependent antitumor mechanisms of PDT become of great importance: the vasculature damage and triggered immune responses are likely to induce the killing of the remaining tumor cells which were not affected by the initial ROS-mediated cytotoxicity. To circumvent this heterogeneity or low target expression, combined targeting of tumor and endothelial cells has been proposed for NBPDT [19], which could likely be expanded to other combinations.

One should not forget that NB-PDT does not overcome all the limitations of conventional PDT, and especially those related to the technology itself still remain. Moreover, NB-PDT faces one additional challenge, and this is that the trust on these small biologicals is only now boosting after the first clinical approval of a NB in 2018 [20]. About a dozen NBs are being evaluated in clinical trials, e.g. as tumor imaging agents (NCT02683083) and as part of engineered anti-tumor T cells (NCT04489862), and encouraging results will definitely aid the further development of NB-PDT at a faster pace.

\section{Potentiating NB-PDT with immune modulation}

The extensive evidence supporting the induction of strong antitumor immune responses by PDT is presented in Chapter $\mathbf{6}$ of this thesis. Likewise, PIT (i.e. antibody-targeted PDT) is also able to trigger antitumor responses in mouse models [21], despite presenting a distinct mechanism to induce immunogenic cell death (ICD) than conventional PDT (i.e. necrosis vs apoptosis) [22]. The initial characterization of these immunological events triggered by NB-PDT is described in Chapter 7, where a similar cell death mechanism to PIT is indicated, suggesting the potential of NB-PDT to stimulate the immune system in a similar manner. Although in vitro data are encouraging, it is clear that further study in in vivo models is required to fully elucidate this aspect of NB-PDT. In fact, these in vivo experiments are ongoing in order to characterize both the systemic reach and the immune memory triggered by NB-PDT.

If the involvement of the immune system in treatment outcome is confirmed for NB-PDT, the next logical step would be to potentiate the treatment by combination with immune modulatory strategies, such as agents to boost the immune system and/or to reverse the immunosuppressive environment. These multimodal approaches are indeed already under clinical evaluation with chemo- and radiotherapy, both capable of acting as ICD inducers [23]. In the context of PDT, only a small number of studies addresses these combinational approaches in the clinic. For HNSCC patients, based on the PDT-induced changes in immune cell populations, the combination of PDT with antibodies to enhance NK cell activation or to release inhibition mediated by $\mathrm{T}$ regulatory cells has been proposed [24]. Of great promise is the sequential application of PDT and imiquimod, a toll-like receptor agonist which stimulates immune responses, resulting in significantly better clinical and histologic response in patients with actinic keratosis [25], basal cell carcinoma [26] and vulvar intraepithelial neoplasia [27]. Therefore, investigating the use of NB-PDT together with imiquimod, first in mouse models, could lead to a successful 
treatment approach. Moreover, the immunity following different targeted PDT approaches has been successfully enhanced with PD1 checkpoint blockade in mice studies $[21,28]$. The combination of EGFR-targeted PIT and anti-PD1 therapy is currently being evaluated in patients with advanced HNSCC or cutaneous SCC (NCT04305795). The results of ongoing clinical trials will definitely contribute to the direction that NBPDT will take. Lastly, special attention should be paid to the illumination protocol since this can impact the development of the PDT-induced antitumor response. In the clinic, the treatment of small surface areas with moderate to high fluences and low fluence rates is considered ideal to boost the associated immune response [29]. These are the PDT regimens that would benefit the most from combinations with immunomodulatory agents.

\section{NB-PDT towards the veterinary clinic}

In Chapter 5, an EGFR-targeting NB-PS conjugate with high PS load was developed with the aim to treat cats with OSCC, an excellent model of human HNSCC. We are currently evaluating the in vivo distribution and specific tumor accumulation of this unique highly loaded conjugate in mice studies. At the same time, efforts are being put into the large-scale production of this conjugate as a veterinary product (e.g. sterilization by filtration, endotoxin quantification and product stability) and arrangements being done to advance NB-PDT to the veterinary clinic. Introducing companion animals with spontaneous cancers in the development of anticancer therapies has proven of value before, as exemplified by the successful story of toceranib phosphate [30]. This was the first drug approved for anticancer targeted therapy in dogs, and the canine studies provided the first evidence for the use of an oral kinase inhibitor as cancer treatment. Its structural analogue, sunitinib, is currently approved as a treatment for several human cancers. This evidences the successful implementation of comparative oncology in the field of targeted therapy to benefit both human and veterinary patients, and we envision a similar scenario for NB-PDT.

Conventional PDT has already been applied in cats with facial SCC with high initial complete response rates $(85 \%)$, but long-term control was achieved only in half of these cases [31]. PDT has proven safe and effective especially for early stage SCCs in cats, while toxicity is encountered when treating large tumors [32]. We believe NB-PDT distinguishes itself from the previous efforts and can yield better outcomes for these veterinary patients with otherwise poor therapeutic options. Moreover, the species crossreactivity of the developed conjugate facilitates further translation of the approach towards the human clinic. The results of this first application of NB-PDT in the veterinary clinic will guide the direction this therapy will take, likely towards the treatment of human cancers currently treated with PDT with relative success, such as HNSCC and basal cell carcinoma.

\section{Conclusion}

The work presented in this thesis forms the basis for the further development of NB-PDT towards the clinic. We have described the application of EGFR-targeted NB-PDT in a clinically relevant model of HNSCC, i.e. patient-derived HNSCC organoids, and presented the efforts to bring NB-PDT to the veterinary clinic to treat cats with OSCC. We also explored the first steps of immune modulation triggered by NB-PDT. It is expected that the ongoing mice studies to explore the implication of the triggered immune responses in the therapeutic outcome of NB-PDT, and the evaluation of NB-PDT in the veterinary clinic, will lead to therapy refinement and enable to exploit the potential of NB-PDT in its full in the clinical setting. 


\section{References}

1. Casas, A.; Di Venosa, G.; Tayyaba, H.; Batlle, A. Mechanisms of Resistance to Photodynamic Therapy. Curr. Med. Chem. 2011, 18, 2486-2515.

2. Marusyk, A.; Janiszewska, M.; Polyak, K. Intratumor Heterogeneity: The Rosetta Stone of Therapy Resistance. Cancer Cell 2020, 37, 471-484.

3. Rosin, F.C.P.; Teixeira, M.G.; Pelissari, C.; Corrêa, L. Resistance of oral cancer cells to 5-ALAmediated photodynamic therapy. J. Cell. Biochem. 2018, 119, 3554-3562.

4. Yano, T.; Muto, M.; Minashi, K.; Iwasaki, J.; Kojima, T.; Fuse, N.; Doi, T.; Kaneko, K.; Ohtsu, A. Photodynamic therapy as salvage treatment for local failure after chemoradiotherapy in patients with esophageal squamous cell carcinoma : A phase II study. Int. J. Cancer 2012, 131, 1228-1234.

5. Nyst, H.J.; Tan, I.B.; Stewart, F.A.; Balm, A.J.M. Is photodynamic therapy a good alternative to surgery and radiotherapy in the treatment of head and neck cancer? Photodiagnosis Photodyn. Ther. 2009, 6, 3-11.

6. Jerjes, W.; Upile, T.; Hamdoon, Z.; Mosse, C.A.; Morcos, M.; Hopper, C. Photodynamic Therapy Outcome for T1/T2 N0 Oral Squamous Cell Carcinoma. Lasers Surg. Med. 2011, 43, 463-469.

7. Ruggiero, E.; Alonso-De Castro, S.; Habtemariam, A.; Salassa, L. Upconverting nanoparticles for the near infrared photoactivation of transition metal complexes: New opportunities and challenges in medicinal inorganic photochemistry. Dalt. Trans. 2016, 45, 13012-13020.

8. Shafirstein, G.; Bellnier, D.; Oakley, E.; Hamilton, S.; Potasek, M.; Beeson, K.; Parilov, E. Interstitial Photodynamic Therapy - A Focused Review. Cancers (Basel) 2017, 9.

9. Fan, W.; Huang, P.; Chen, X. Overcoming the Achilles' heel of photodynamic therapy. Chem. Soc. Rev. 2016, 45, 6488-6519.

10. Mitsunaga, M.; Ogawa, M.; Kosaka, N.; Rosenblum, L.T.; Choyke, P.L.; Kobayashi, H. Cancer Cell-Selective In Vivo Near Infrared Photoimmunotherapy Targeting Specific Membrane Molecules. Nat. Med. 2011, 17, 1685-1691.

11. David M. Cognetti; Johnson, J.M.; Curry, J.M.; Mott, F.; Kochuparambil, S.T.; McDonald, D.; Fidler, M.J.; Stenson, K.; Vasan, N.R.; Razaq, M.; et al. Results of a phase 2a, multicenter, openlabel, study of RM-1929 photoimmunotherapy (PIT) in patients with locoregional, recurrent head and neck squamous cell carcinoma (rHNSCC). J. Clin. Oncol. 2019, 37, 6014.

12. Heukers, R.; van Bergen en Henegouwen, P.M.P.; Oliveira, S. Nanobody-photosensitizer conjugates for targeted photodynamic therapy. Nanomedicine Nanotechnology, Biol. Med. 2014, 10, 1441-1451.

13. Van Driel, P.B.A.A.; Boonstra, M.C.; Slooter, M.D.; Heukers, R.; Stammes, M.A.; Snoeks, T.J.A.; De Bruijn, H.S.; Van Diest, P.J.; Vahrmeijer, A.L.; Van Bergen En Henegouwen, P.M.P.; et al. EGFR targeted nanobody-photosensitizer conjugates for photodynamic therapy in a pre-clinical model of head and neck cancer. J. Control. Release 2016, 229, 93-105.

14. Krasniqi, A.; Bialkowska, M.; Xavier, C.; Jeught, K. Van Der; Muyldermans, S.; Devoogdt, N.; Huyvetter, M.D. Pharmacokinetics of radiolabeled dimeric sdAbs constructs targeting human CD20. N. Biotechnol. 2018, 45, 69-79.

15. Debie, P.; Lafont, C.; Defrise, M.; Hansen, I.; Van Willigen, D.M.; Van Leeuwen, F.W.B.; Gijsbers, R.; DHuyvetter, M.; Devoogdt, N.; Lahoutte, T.; et al. Size and affinity kinetics of nanobodies influence targeting and penetration of solid tumours. J. Control. Release 2020, 317, 34-42.

16. Deken, M.M.; Kijanka, M.M.; Beltrán Hernández, I.; Slooter, M.D.; de Bruijn, H.S.; van Diest, P.J.; van Bergen en Henegouwen, P.M.; Lowik, C.W.; Robinson, D.J.; Vahrmeijer, A.L.; et al. Nanobody-targeted photodynamic therapy induces significant tumor regression of trastuzumabresistant HER2-positive breast cancer, after a single treatment session. J. Control. Release 2020, 323, 269-281.

17. Hudson, R.; Carcenac, M.; Smith, K.; Madden, L.; Clarke, O.; Pelegrin, A.; Greenman, J.; Boyle, R.W. The development and characterisation of porphyrin isothiocyanate-monoclonal antibody conjugates for photoimmunotherapy. Br. J. Cancer 2005, 92, 1442-1449.

18. Jiang, X.; Ye, J.; Dong, Z.; Hu, S.; Xiao, M. Novel genetic alterations and their impact on target 
therapy response in head and neck squamous cell carcinoma. Cancer Manag. Res. 2019, 11, 13211336.

19. Mashayekhi, V.; Xenaki, K.T.; van Bergen en Henegouwen, P.M.P. Van; Oliveira, S. Dual Targeting of Endothelial and Cancer Cells Potentiates In Vitro Nanobody-Targeted Photodynamic Therapy. Cancers (Basel) 2020, 12, 2732.

20. Morrison, C. Nanobody approval gives domain antibodies a boost. Nat. Rev. Drug Discov. 2019, $18,485-487$.

21. Nagaya, T.; Friedman, J.; Maruoka, Y.; Ogata, F.; Okuyama, S.; Clavijo, P.E.; Choyke, P.L.; Allen, C.; Kobayashi, H. Host immunity following near-infrared photoimmunotherapy is enhanced with PD-1 checkpoint blockade to eradicate established antigenic tumors. Cancer Immunol. Res. 2019, 7, 401-413.

22. Ogawa, M.; Tomita, Y.; Nakamura, Y.; Lee, M.; Lee, S.; Tomita, S.; Nagaya, T.; Sato, K.; Yamauchi, T.; Trepel, B.; et al. Immunogenic cancer cell death selectively induced by near infrared photoimmunotherapy initiates host tumor immunity. Oncotarget 2017, 8, 10425-10436.

23. Rapoport, B.L.; Anderson, R. Realizing the Clinical Potential of Immunogenic Cell Death in Cancer Chemotherapy and Radiotherapy. Int. J. Mol. Sci. 2019, 20, 959.

24. Theodoraki, M.N.; Lorenz, K.; Lotfi, R.; Fürst, D.; Tsamadou, C.; Jaekle, S.; Mytilineos, J.; Brunner, C.; Theodorakis, J.; Hoffmann, T.K.; et al. Influence of photodynamic therapy on peripheral immune cell populations and cytokine concentrations in head and neck cancer. Photodiagnosis Photodyn. Ther. 2017, 19, 194-201.

25. Serra-Guillén, C.; Nagore, E.; Hueso, L.; Traves, V.; Messeguer, F.; Sanmartín, O.; Llombart, B.; Requena, C.; Botella-Estrada, R.; Guillén, C. A randomized pilot comparative study of topical methyl aminolevulinate photodynamic therapy versus imiquimod $5 \%$ versus sequential application of both therapies in immunocompetent patients with actinic keratosis: Clinical and histologic outcomes. J. Am. Acad. Dermatol. 2012, 66, 131-137.

26. Osiecka, B.J.; Jurczyszyn, K.; Ziółkowski, P. The application of Levulan ${ }^{\circledR}$-based photodynamic therapy with imiquimod in the treatment of recurrent basal cell carcinoma. Medidcal Sci. Monit. 2012, 18, 5-9.

27. Winters, U.; Daayana, S.; Lear, J.T.; Tomlinson, A.E.; Elkord, E.; Stern, P.L.; Kitchener, H.C. Clinical and immunologic results of a phase II trial of sequential imiquimod and photodynamic therapy for vulval intraepithelial neoplasia. Clin. Cancer Res. 2008.

28. Bao, R.; Wang, Y.; Lai, J.; Zhu, H.; Zhao, Y.; Li, S.; Li, N.; Huang, J.; Yang, Z.; Wang, F.; et al. Enhancing Anti-PD-1/PD-L1 Immune Checkpoint Inhibitory Cancer Therapy by CD276-Targeted Photodynamic Ablation of Tumor Cells and Tumor Vasculature. Mol. Pharm. 2019, 16, 339-348.

29. Beltrán Hernández, I.; Yu, Y.; Ossendorp, F.; Korbelik, M.; Oliveira, S. Preclinical and Clinical Evidence of Immune Responses Triggered in Oncologic Photodynamic Therapy: Clinical Recommendations. J. Clin. Med. 2020, 9, 333 .

30. Regan, D.; Garcia, K.; Thamm, D. Clinical, Pathological, and Ethical Considerations for the Conduct of Clinical Trials in Dogs with Naturally Occurring Cancer: A Comparative Approach to Accelerate Translational Drug Development. ILAR J. 2018, 59, 99-110.

31. Bexfield, N.H.; Stell, A.J.; Gear, R.N.; Dobson, J.M. Photodynamic Therapy of Superficial Nasal Planum SquamousCell Carcinomas in Cats: 55 Cases. J Vet Intern Med 2008, 22, 1385-1389.

32. Magne, M.L.; Rodriguez, C.O.; Autry, S.A.; Edwards, B.F.; Theon, A.P.; Madewell, B.R. Photodynamic therapy of facial squamous cell carcinoma in cats using a new photosensitizer. Lasers Surg. Med. 1997, 20, 202-209. 
Summary and perspectives 



\section{APPENDICES}

Nederlandse samenvatting

Curriculum Vitae

List of publications

Acknowledgements 


\section{Nederlandse samenvatting}

Mainstream therapeutische behandelingen tegen kanker gaan vaak gepaard met bijwerkingen en hebben een aanzienlijke impact op de kwaliteit van leven van patiënten. Er zijn inspanningen geleverd in de richting van nieuwe tumorselectieve behandelingen, met het idee om schade aan gezonde weefsels te minimaliseren. In dit proefschrift richten we ons op een aanpak die in de afgelopen 7 jaar is ontwikkeld: nanobody gebaseerde fotodynamische therapie (NB-PDT). Deze gerichte aanpak maakt gebruik van nanobodies (NBs), de kleinste natuurlijk voorkomende antigeen bindende domeinen. De NBs zorgen voor de selectieve aflevering van een fotosensibilisator (PS) aan de tumorcellen, een molecuul dat bij lokale activering door licht leidt tot de vorming van reactieve zuurstofverbindingen en de daaruit voortvloeiende celdood. NB-PDT heeft tot nu toe aangetoond veelbelovend te zijn in vitro en in orthotopische muis modellen gebruik makend van verschillende moleculaire targets. Dit proefschrift richt zich op de volgende stap, namelijk NB-PDT een stap dichter bij de kliniek te brengen.

In Hoofdstuk 1 wordt fotodynamische therapie (PDT) gepresenteerd als een tumorselectieve en minimaal invasieve strategie, die echter nauwelijks gebruikt wordt in de kliniek. Pogingen om PDT te verbeteren door de invoering van een targeting component worden besproken, namelijk antilichaam gebaseerde PDT (de zogenaamde foto-immuuntherapie of PIT) en NB-PDT. PIT wordt momenteel geëvalueerd in een fase III klinische studie voor de behandeling van hoofd-hals plaveiselcelcarcinoom (HNSCC), met behulp van een water oplosbare PS geconjugeerd aan cetuximab, een commercieel monoklonaal antilichaam (mAb) gericht op de epidermale groeifactor receptor (EGFR). Gebruikmaken van een klein targeting molecuul zoals een NB levert voordelen op, en kan leiden tot een stap voorwaarts in de verbetering van PDT. Die voordelen houden in dat er een snellere en homogenere verdeling over tumorweefsel is waardoor een snellere start van de lichttoepassing na toediening van de PS mogelijk is, en een snelle nierklaring waardoor de kans op lichtgevoeligheid na de behandeling verminderd wordt. HNSCC en de aanwezigheid van EGFR in deze vorm van kanker worden ook besproken in dit hoofdstuk, als focus voor de verdere ontwikkeling van NB-PDT.

Een beperkend aspect van PIT is de heterogene verdeling van het mAb-PS conjugaat in de tumor vanwege het grote moleculaire gewicht van de $\mathrm{mAb}(\sim 150 \mathrm{kDa})$. NBs zijn daarentegen klein $(\sim 15 \mathrm{kDa})$, en ook makkelijk te ontwerpen als multivalente constructen. Daarom onderzochten we in Hoofdstuk 2 het effect van moleculair gewicht van verschillende EGFR-targeting NBs op de tumorpenetratie, vergeleken met een $\mathrm{mAb}$, met behulp van tumorsferoïden en tumordragende muizen. Dual-isotoop computertomografie met behulp van emissie van enkelvoudige fotonen (SPECT) werd voor de eerste keer gebruikt voor NBs, waardoor longitudinale en gelijktijdige detectie van twee NBs in een enkel dier mogelijk was. Deze studie toonde aan dat het verhogen van de grootte van het NB-formaat, zelfs met slechts $15 \mathrm{kDa}$, de tumorpenetratie en distributie belemmert. Het wordt in het bijzonder duidelijk dat NBs in hun monomere vorm een superieure keuze zijn voor moleculaire imaging en, waarschijnlijk, ook voor gerichte PDT.

In Hoofdstuk 3 zijn we in de richting gegaan van het gebied van gerichte PDT en het gebruik van klinisch relevante 3D-modellen met translationeel potentieel: organoïden gekweekt uit zowel HNSCC-patiëntmateriaal als overeenkomstig normaal weefsel. Ten eerste bleek de EGFR-expressie van de organoïden overeen te komen met het primaire weefsel, een zeer belangrijk kenmerk omdat veel gebruikte HNSCC-cellijnen vaak afwijkende expressieniveaus hebben. De potentie van EGFR-gerichte PDT werd vervolgens onderzocht met behulp van verschillende NB-PS constructen en cetuximab- 
PS. De organoïde technologie staat toe om de specificiteit van gerichte PDT te verkennen door het vergelijken van overeenkomende tumoren en normale organoïden. De EGFR expressie was immers lager in de normale organoïden, en deze werden dan ook niet beïnvloed door de behandeling. In het algemeen was NB-PDT effectiever in het induceren van celdood dan PIT, hetgeen de superioriteit van NBs voor gerichte PDT aantoont. Belangrijk is dat deze studie HNSCC-organoïden presenteerde als een realistischere weergave van tumoren dan veelgebruikte cellijnen.

De bemoedigende resultaten van NB-PDT werkzaamheid in HNSCC organoïden leidde tot de verdere ontwikkeling van NB-PDT naar de humane kliniek. Derhalve onderzoekt Hoofdstuk 4 de introductie van gezelschapsdieren met spontane tumoren in de huidige programma's voor geneesmiddelenontwikkeling om therapieverfijning te ondersteunen en het translationele succes van gerichte therapieën tegen kanker te vergroten. Tegelijkertijd krijgen deze veterinaire patiënten toegang tot nieuwe, anders ontoegankelijke therapieën. Dit hoofdstuk geeft een overzicht van zogenaamde targetbare membraaneiwitten die geëxpresseerd zijn in de beste gekarakteriseerde hond- en katachtige solide tumor modellen, die sterk lijken op de verwante menselijke maligniteiten. EGFR werd geïdentificeerd als een kandidaat target voor de ontwikkeling van gerichte therapieën tegen kat OSCC, dat wordt beschouwd als een natuurlijk voorkomend model van de menselijke HNSCC en waarvoor tot nu toe geen curatieve behandeling is. Daarom werden inspanningen geleverd in de ontwikkeling van een NBPS conjugaat voor de behandeling van katten met OSCC. Het in vitro werk dat aan deze stap in de dierenkliniek voorafgaat, wordt gepresenteerd in Hoofdstuk 5. Een NB werd geïdentificeerd met het vermogen om aan zowel menselijk als katachtig EGFR te binden met vergelijkbare affiniteit. Om krachtige cytotoxiciteit te induceren in cellen met klinisch relevante EGFR-expressieniveaus, werd een zeer cytotoxisch NB-PS-conjugaat ontwikkeld (d.w.z. NiBh) bestaande uit een monomeer NB, het ideale formaat zoals in hoofdstuk 2 beschreven. NiBh werd met succes gebruikt om krachtige celdood van kat OSCC cellijnen met hoge specificiteit te veroorzaken, aangezien de aangrenzende cellen met lage EGFR expressieniveaus ongedeerd bleven. De bemoedigende resultaten van deze studie ondersteunen het idee om NiBh verder te ontwikkelen voor de behandeling van katten met OSCC.

Een klinisch relevant kenmerk van PDT is het vermogen om antitumor immuunreacties te activeren, die essentieel zijn voor het creëren van een systemische reactie om metastase te bestrijden en recidieven te voorkomen. Hoe veelbelovend dit ook is, desalniettemin is het antitumor mechanisme van PDT is nog niet volledig opgehelderd en wordt het niet altijd met succes geactiveerd. Hoofdstuk 6 bespreekt het preklinische en klinische bewijs met betrekking tot dit aspect van PDT. In het algemeen wordt altijd eerst een lokale ontsteking geactiveerd, die zich verder kan ontwikkelen tot een adaptieve immuunrespons wanneer immunogene celdood (ICD) is geïnduceerd. ICD omvat de herverdeling van stervende tumorcellen en het vrijkomen van schade-geassocieerde moleculaire patronen (DAMPs) hieruit, die worden herkend door infiltrerende immuuncellen die vervolgens antitumorreacties kunnen activeren en herstellen. Het is dus duidelijk dat het activeren van immuunreacties sterk kan bijdragen aan de werkzaamheid van PDT. Daarom richt Hoofdstuk 7 zich op de beoordeling van de mogelijke immuunstimulatie veroorzaakt door NB-PDT in vitro. Verschillende indicatoren van ICD werden gedetecteerd na NB-PDT op tumorcellen. Die indicatoren geven aan dat de NBPDT in staat is om rijping van dendritische cellen en de daaropvolgende T-cel activering te induceren. Dit zijn de eerste stappen die wijzen op immuunstimulatie veroorzaakt door NB-PDT, die vervolgens kunnen resulteren in een mogelijk systemisch effect na een eerste lokale behandeling. 
Alles tezamen, benadrukt dit proefschrift de waarde van NBs ten opzichte van mAbs om een snelle en homogene verdeling over tumoren te bereiken, presenteert het de potentie van NB-PDT in een klinisch relevant model bestaande uit HNSCC-organoïden, en beschrijft het de in vitro stappen naar de toepassing van NB-PDT om katten met OSCC te behandelen. Bovendien worden de eerste aanwijzingen van immuunstimulatie door NB-PDT beschreven, wat wijst op de inductie van een antitumormechanisme met grote klinische implicaties. 


\section{Curriculum vitae}

Irati Beltrán Hernández was born on the $24^{\text {th }}$ of March in 1992 in Ibiza, Spain. After obtaining her high school diploma, she obtained her bachelor degree in Biotechnology from the Autonomous University of Barcelona, in Spain. In 2014, she enrolled in the Drug Innovation master program at Utrecht University, in the Netherlands. During this time, she worked in projects related to the use of different formats of nanobodies and antibodies, including the application of bispecific antibodies for anticancer therapy, nanobodies for imaging and the incorporation of nanobodies as targeting ligands in extracellular vesicles. After completing her masters, she continued her studies at Utrecht University, starting her $\mathrm{PhD}$ at the lab of Dr. Sabrina Oliveira in November 2016. The results of her work are presented in this thesis. She is currently working as a post-doc in a collaboration effort between the group of Dr. Sabrina Oliveira and Prof. Dr. Jurgen Kuball (Tumor immunologist, UMCU), aiming to characterize the murine antitumor immunity triggered by nanobody-targeted photodynamic therapy. In addition, she continues to collaborate with Bas van Nimwegen and Erik Teske from the Veterinary Clinic of the Faculty of Veterinary Medicine to apply this therapy in client owned cats with oral cancer. 


\section{List of publications}

I. Beltrán Hernández, R. Rompen, R. Rossin, K.T. Xenaki, E.A. Katrukha, K. Nicolay, P. van Bergen en Henegouwen, H. Grüll, S. Oliveira. Imaging of tumor spheroids, dual-isotope SPECT, and autoradiographic analysis to assess the tumor uptake and distribution of different nanobodies. Mol. Imaging Biol. 2019, 21, 1079-1088

E. Driehuis*, S. Spelier*, I. Beltrán Hernández, R. De Bree, S.M. Willems, H. Clevers, S. Oliveira. Patient-derived head and neck cancer organoids recapitulate EGFR expression levels of respective tissues and are responsive to EGFR-targeted photodynamic therapy. J. Clin. Med. $2019,8,1880$

I. Beltrán Hernández*, J. Kromhout*, E. Teske, W.E. Hennink, S.A. van Nimwegen, S. Oliveira. Molecular targets for anticancer therapies in companion animals and humans: what can we learn from each other? Theranostics 2021, in press

I. Beltrán Hernández*, Y. Yu*, F. Ossendorp, M. Korbelik, S. Oliveira. Preclinical and clinical evidence of immune responses triggered in oncologic photodynamic therapy: clinical recommendations. J. Clin. Med. 2020, 9, 333

I. Beltrán Hernández, M.L. Angelier, T. Del Buono D’Ondes, A. Di Maggio, Y. Yu, S. Oliveira. The potential of nanobody-targeted photodynamic therapy to trigger immune responses. Cancers (Basel) 2020, 12, 978

M.M. Deken*, M.M. Kijanka*, I. Beltrán Hernández, M.D. Slooter, P.J. van Diest, H.S. de Bruijn, P.M. van Bergen en Henegouwen, C.W. Lowik, D.J. Robinson, A.L. Vahrmeijer, S. Oliveira. Nanobody-targeted photodynamic therapy induces significant tumor regression of trastuzumab-resistant HER2-positive breast cancer, after a single treatment session. J. Control. Release 2020, 323, 269-281

Y. Liu, L. Scrivano, J.D. Peterson, M.H.A.M Fens, I. Beltrán Hernández, B. Mesquita, J. Sastre Torano, W.E. Hennink, C.F. van Nostrum, S. Oliveira. EGFR-targeted nanobody functionalized polymeric micelles loaded with mTHPC for selective photodynamic therapy. Mol. Pharm 2020, 17, 1276-1292.

P.J. Engelberts, I.H. Hiemstra, B. de Jong, D.H. Schuurhuis, J. Meesters, I. Beltrán Hernández, S.C. Oostindie, J. Neijssen, E.N. van den Brink, G.J. Horbach, S. Verploegen, A.F. Labrijn, T. Salcedo, J. Schuurman, P.W.H.I. Parren, E.C.W. Breij. DuoBody-CD3xCD20 induces potent Tcell-mediated killing of malignant $\mathrm{B}$ cells in preclinical models and provides opportunities for subcutaneous dosing. EBioMedicine 2020, 52, 102625

R. Damman, A. Lucini Paioni, K.T. Xenaki, I. Beltrán Hernández, P.M.P. van Bergen en Henegouwen, M. Baldus. Development of in vitro-grown spheroids as a 3D tumor model system for solid-state NMR spectroscopy. J. Biomol. NMR 2020, 74,401-412 


\section{Acknowledgements}

A lot has happened in the past 4 years, not only inside the lab, but also outside. Each of those experiences and people involved have made it possible for me to finish my $\mathrm{PhD}$ project and, most importantly, made me enjoy it along the way.

First, I would like to thank my supervisor and co-promotor Sabrina. Our history goes back to my master internship, when you already showed me the warmhearted and understanding person you are. This combined with your scientific knowledge and view in the clinic reassured me that doing a $\mathrm{PhD}$ by your side would be a magnificent journey. This was indeed the case. From the beginning, you welcomed my ideas and let me find my way. You were always there when I was blocked and needed new and brilliant insights. With your positive attitude upon new challenges, you kept me motivated and moving forward. Also remarkable is your kindness and your interest in me having a good work-life balance. I admire your dedication to your work and your family. I am really thankful for all your support and all you have taught me in the lab and outside.

I also appreciate the input of Paul, my second co-promotor. We had regular meetings and exchanged a lot of ideas. Although we sometimes had different views on things, this proved very valuable since it made me reconsider and improve my approaches. I thank Wim, as my promotor, for all your suggestions and feedback on my different projects. It was clear to me that you always aimed at a piece of work of the highest quality, and that kept pushing me in that direction. I also thank Anna, my second promotor, for her support and feedback during our meetings.

The work presented in this thesis would not have been possible without the effort of many collaborators. In particular, I would like to thank the veterinarians and veterinary researchers Bas, Erik, Guy, Jan and Jan Willem. I learned a lot during our meetings, especially how to guide research from the clinical perspective and with the interest of the animal as a priority. I hope soon we manage to advance our project and reach the veterinary patients. Adri and Elpetra, thank you for your help and guidance with new experiments in the veterinary department. Else and Sacha, it was great to learn about the organoid technique from you and it was very motivating working with two such rigorous and hard-working people.

During the 4 years of $\mathrm{PhD}$, I got the chance to supervise several bachelor and master students whose work and effort have contributed to the completion of this thesis: Mathieu, Sacha, Xavi, Alessia, Tommaso, Nasia, Julien, Frederique, Dan, Charlotte and Glenn. I also thank Jannes and Christina, whose writing assignments helped with the preparation of a grant and a review regarding the studies in the veterinary clinic. I learnt a lot as a scientist and as a person while supervising all of you, and I thank you all for the positive attitude and nice moments together.

I am so glad that Sabrina's group is in such close contact with Paul's group. We all together get to discuss our scientific work during meetings, but also have a lot of fun outside the lab. Katerina, Sebas, Sofia and Erik, you were the best office colleagues one could wish for. Sometimes too noisy, but totally worth it because of all the fun, laughs, (scientific) debates, drama, gossip, arm wrestling, etc. Iris and Sara, although not directly working with you, always nice to have two very kind and sweet persons around. Yingxin, I learnt a lot from you about the immune system and designing complex experiments. Bárbara, I am very glad that you joined the group. Your constant smile made me happy even on stressful days and we both understood the struggles of running from building to building. Vida, we have gone together through our $\mathrm{PhD}$ journeys, from beginning to end. You have helped me a thousand times in the lab when I was running too many things in parallel, and always did it with a smile without expecting anything in return. I really 
appreciate that. We have shared frustrations, supported each other and kept pushing. You and Kamal have also been very good friends outside the lab.

It makes me immensely happy that I successfully managed to combine my $\mathrm{PhD}$ work with playing (professional) basketball. Although physically and psychologically demanding sometimes, I don't regret the decision for a second. I believe this combination and balance kept me going and allowed me to hide in a basketball bubble and forget the challenges in the lab. I am really grateful for every moment inside and outside the court. I have had excellent teammates who have become really good friends. Sanne, Ciska and all other current and past teammates, thank you all for putting a smile on my face every training and game. Alba, I have laughed a lot with you, your funny expressions, silly videos and gossiping in Spanish.

A special mention to my dear friends from Ibiza. Despite being far away and all in different parts of the globe, we kept contact, shared problems, supported each other and enjoyed very much when we gathered together.

My family has always supported me and believed in me to achieve all I wanted, even when that means going far from home. Ama, especialmente tú, siempre has cuidado de mí con amor y me has hecho la persona que soy hoy. Tú crees más en mí de lo que yo lo hago, y eso siempre me ha hecho querer ser la mejor versión de mí misma. Aita, de ti he sacado la inquietud de siempre querer saber más y eso me ha llevado a donde estoy hoy. Aprecio el interés que los dos siempre habéis mostrado en mi trabajo, incluso cuando resulta tremendamente complicado entender de qué estoy hablando. Paco, el amor y cariño incondicional que me has mostrado desde el principio ha sido muy importante para mí. Estoy donde estoy gracias a ti también. Tineke en Arie, ik zie jullie als mijn tweede ouders, jullie hebben van me gehouden en hebben voor mij gezorgd vanaf het moment dat ik in jullie leven verscheen. Er zijn geen woorden voor hoeveel ik alles waardeer wat jullie voor mij gedaan hebben de afgelopen jaren.

Rowan, you have been my rock throughout this journey, with your love, support, advice and patience. I admire how you are always helping and taking care of everybody around you out of pure kindness, you have made me a better person. We have gone through so much together and you have brought so much happiness to my life. I cannot wait to see what the future holds for us. Te quiero. 
